\title{
UTILIZATION OF GOLD CONTAINING NANOMATERIALS FOR TARGETED RADIOTHERAPEUTIC AND DIAGNOSTIC APPLICATIONS
}

\author{
A Dissertation \\ Presented to
}

The Faculty of the Graduate Committee

University of Missouri-Columbia

In Partial Fulfillment of the Requirements for the Degree

Doctor of Philosophy

By MARK F. MCLAUGHLIN

Dr. J. David Robertson, Committee Chair

MAY 2013 
The undersigned, appointed by the dean of the Graduate School, have examined the dissertation entitled:

\section{UTILIZATION OF GOLD CONTAINING NANOMATERIALS FOR TARGETED RADIOTHERAPEUTIC AND DIAGNOSTIC APPLICATIONS}

Presented by Mark F. McLaughlin,

A candidate for the degree of Doctor of Philosophy,

And hereby certify that, in their opinion, it is worthy of acceptance.

Dr. J. David Robertson

Dr. Cathy Cutler

Dr. Silvia S. Jurisson

Dr. Saed Mirzadeh

Dr. Steven W. Keller 
For my parents, who nurtured me into the man I am today and provided such stunning examples of selfless love.

For my brother, who never stopped serving as my role model. 


\section{Acknowledgements}

No research occurs in a vacuum, except pyrophoric research. I have been blessed to have able and willing collaborators in my research throughout my graduate studies. Primarily I would like to thank my advisor, Dr. Robertson, for his passion, patience, and encouragement. Dr. Robertson truly loves the field of radiochemistry and inspires that same love in those fortunate enough to work for him. He always makes time for his students despite his busy schedule and has a knack for explaining difficult concepts in understandable terms. I also owe a deep debt of gratitude to Dr. Jurisson, who first exposed me to the field of radiochemistry and later served as a committee member for my Ph.D. Her insistence that I gain a better understanding of biological interactions and targeting made me a better radiopharmaceutical scientist. I would also like to thank Dr. Cutler for serving on my committee and directing my research into gold nanoparticles and Dr. Keller for helping me to better understand the complexities of nanoparticles. Finally, I would like to thank Dr. Mirzadeh for serving on my committee and directing my research in Oak Ridge. Dr. Mirzadeh offered a wealth of experience and hospitality during my time in Tennessee for which I am forever grateful.

I also received a good deal of instruction and assistance in running the many analytical instruments used in this manuscript. Dr. Ajit Zambre provided Au-DTDTPA and aided DLS nanoparticle characterization. Missy Evans-Blumer organized and ran countless samples for NAA. Terry Carmack and Lisa Watkinson performed wonderful 
biodistribution studies with my many gold nanoparticle systems. Dr. Adam Rondinone taught me how to use the TEM and XRD facilities at Oak Ridge and was a wonderful resource of nanoparticle knowledge. Dr. Rose Boll and Karen Murphy were invaluable in accommodating my many and ceaseless requests for ${ }^{225} \mathrm{Ac}$. Dr. Jonathan Woodward introduced me to the $\mathrm{LnPO}_{4}$ synthesis and showed me the ropes of working in the labs at Oak Ridge. Dr. Steve Kennel graciously performed our ${ }^{225} \mathrm{Ac}$ animal trials and provided a model of healthy skepticism that greatly improved the rigor and quality of my scientific work. Dr. Martin Brechbiel and Diane Milenic were influential in the labeling and characterization of the panitumumab nanoparticle system. Finally, I would like to thank Dr. Paul Pevsner for turning me into a mouse surgeon and inspiring my career in research medicine. Dr. Pevsner's generosity is matched only by his energy and amiability. 


\section{TABLE OF CONTENTS}

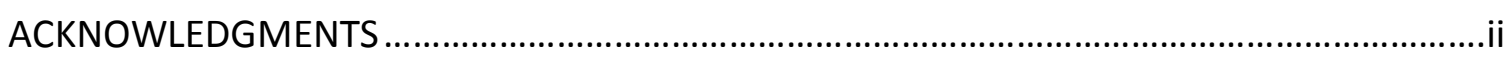

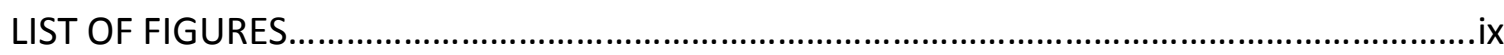

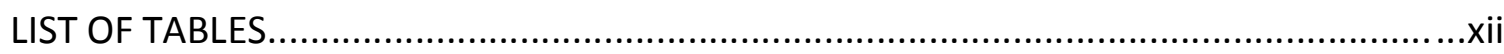

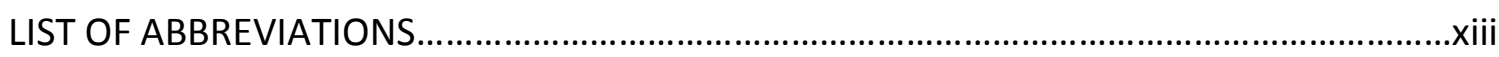

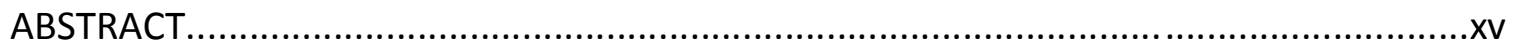

CHAPTER 1: INTRODUCTION TO NANOPARTICLE BASED RADIATION THERAPY.................1

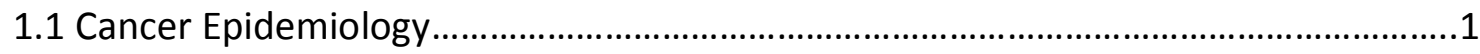

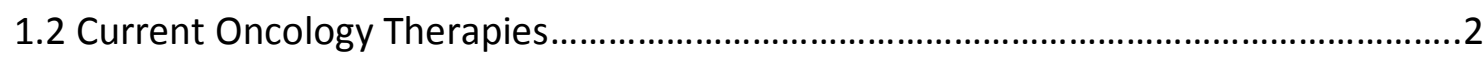

1.3 Targeted Radiotherapies and Types of Therapeutic Radiation ..................................3

1.4 Nanoparticles for Cancer Therapy.........................................................................

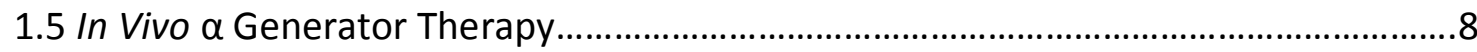

CHAPTER 2: GOLD NANOPARTICLES FOR THERAPY OF UNRESECTABLE PROSTATE

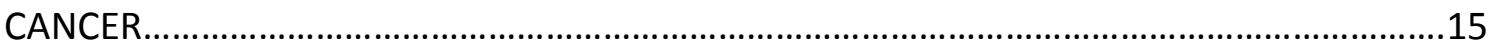

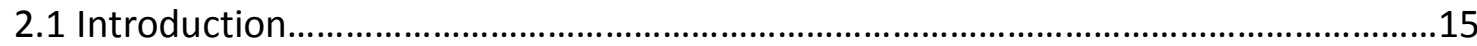

2.1.1 Production, Separation, and Nuclear Properties of Radiogold...........................15

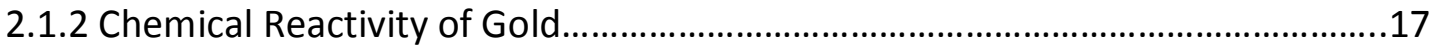

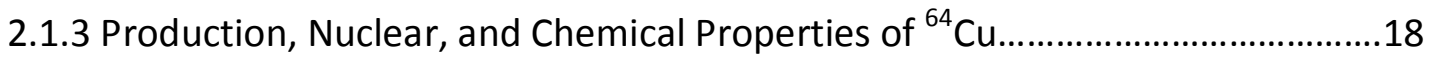

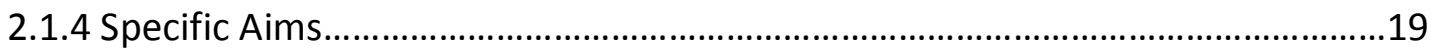

2.2 Gum Arabic Au Nanoparticle Experimental.......................................................21

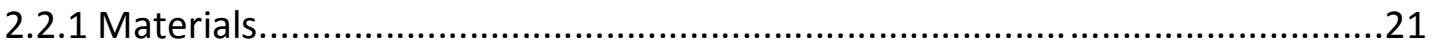

2.2.2 Gum Arabic Gold Nanoparticle Synthesis..............................................21 
2.2.3 Biodistribution and Animal Studies

2.3 Gum Arabic Gold Nanoparticle Results and Discussion....................................23

2.4 Starch-Bombesin Gold Nanoparticle Experimental.......................................27

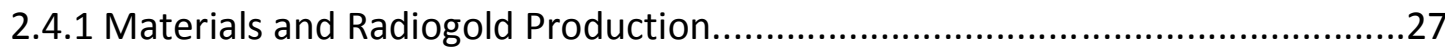

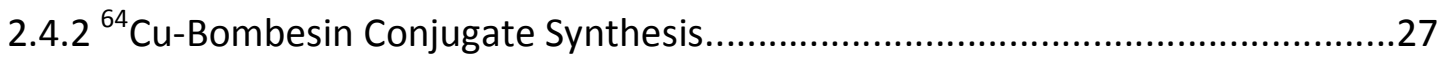

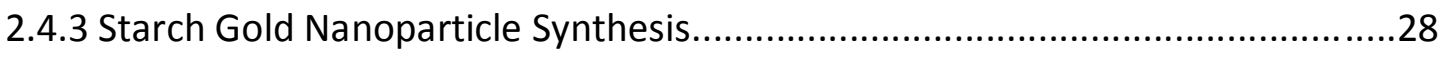

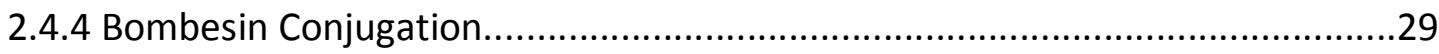

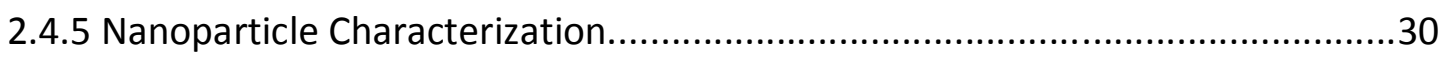

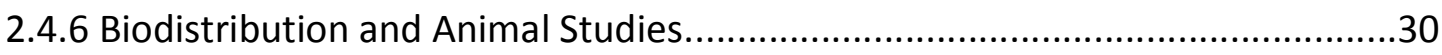

2.5 Starch-Bombesin Gold Nanoparticle Results and Discussion.............................33

2.5.1 Starch Gold Nanoparticle Yield and Characterization....................................33

2.5.2 Gum Arabic Gold Nanoparticle Yield and Characterization............................35

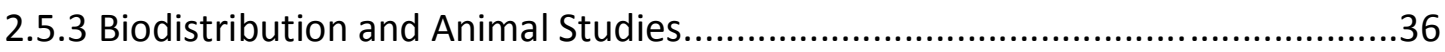

$2.6{ }^{64} \mathrm{Cu}$-DTDTPA Gold Nanoparticle Experimental...............................................42

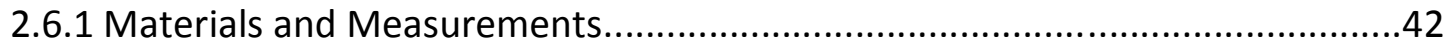

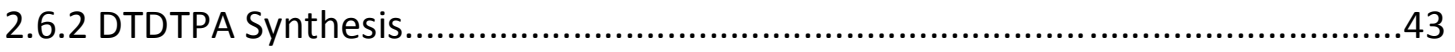

2.6.3 Synthesis of DTDTPA Gold Nanoparticle Conjugates...................................45

2.6.4 Chelation of ${ }^{64} \mathrm{Cu}$ with Gold-DTDTPA Nanoparticles.....................................45

2.6.5 Titrimetric Analysis of Copper on Gold-DTDTPA Nanoparticles......................46

2.6.6 ${ }^{64} \mathrm{Cu}$ Isotope Production and Specific Activity Analysis...................................46

2.6.7 Radiochemical Synthesis of ${ }^{64} \mathrm{Cu}$ Gold-DTDTPA Nanoparticles........................45

2.6.8 In Vitro Stability of Gold-DTDTPA and ${ }^{64} \mathrm{Cu}$ Gold-DTDTPA Nanoparticles..........47

2.6.9 In Vitro Cytotoxicity Measurements........................................................48

2.6.10 In Vivo Biodistribution Measurements...................................................49

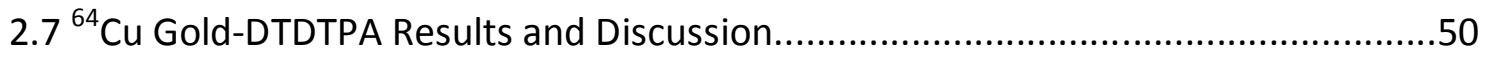


CHAPTER 3: GOLD SHELL NANOPARTICLES FOR in vivo ${ }^{225} \mathrm{Ac} \alpha$-GENERATOR .59

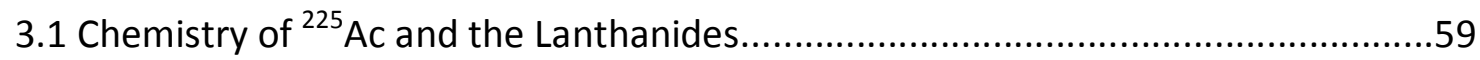

3.2 Nanoparticle Synthesis and Characterization Experimental.............................60

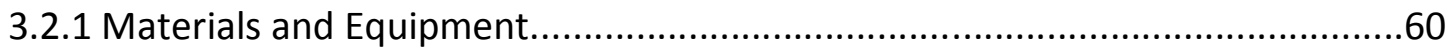

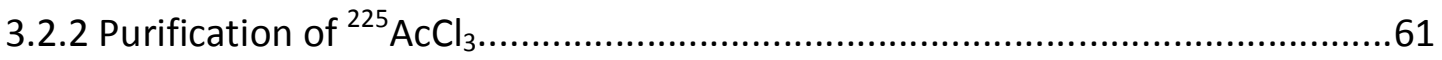

3.2.3 Synthesis of $\left\{\mathrm{La}_{x} \mathrm{Gd}_{1-\mathrm{x}}\right\} \mathrm{PO}_{4}$ nanoparticle cores......................................62

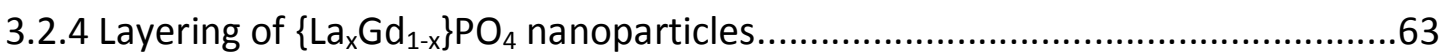

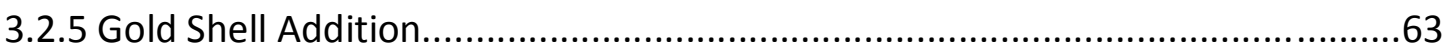

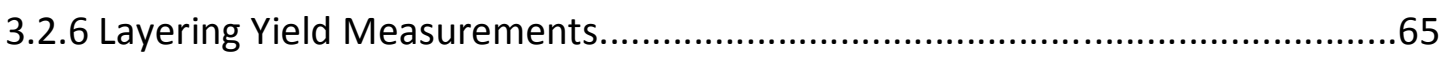

3.2.7 In Vitro Stability Studies...........................................................................67

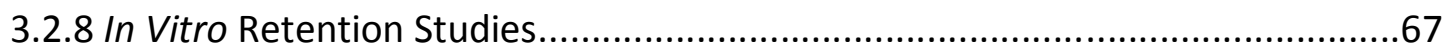

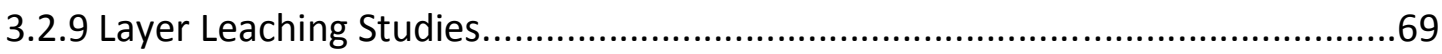

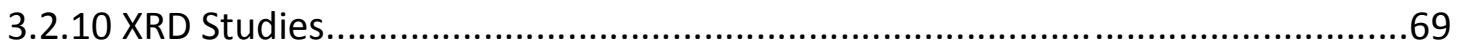

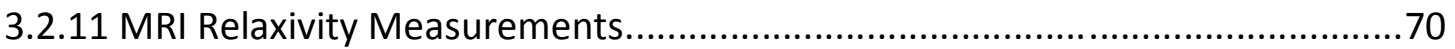

3.3 Nanoparticle Characterization Results and Discussion...................................70

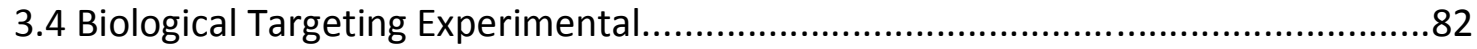

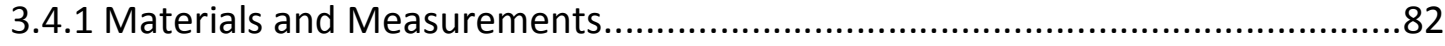

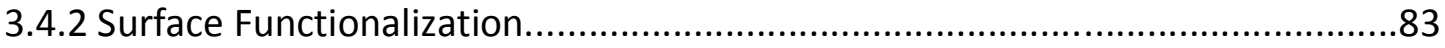

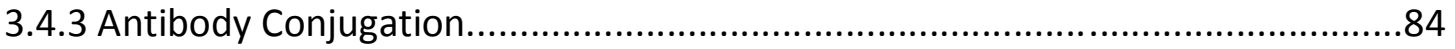

3.4.4 In Vitro Antibody Quantification and Competition Assay.................................85

3.4.5 Radiotracer Antibody Saturation Studies and Biodistribution........................87

3.4.6 Biodistribution and In Vivo Retention Studies with

$\left\{\mathrm{La}_{0.5} \mathrm{Gd}_{0.5}\right\} \mathrm{PO}_{4} @ 4 \mathrm{GdPO}_{4} @ A u-P E G-m A b$ 201b....................................................88

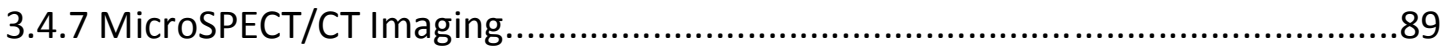

3.4.8 Effect of Clodronate Liposomes on Nanoparticle Biodistribution and Uptake..90 
3.4.9 Electron Microscopy Studies......................................................................91

3.4.10 EMT-6 Therapy Study...............................................................................91

3.4.11 In Vivo Evaluation of Blood Brain Barrier Penetration with Anti-GFAP

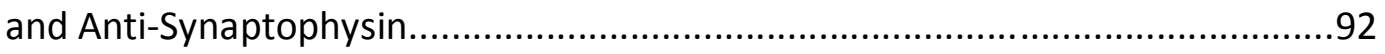

3.5 Biological Targeting Results and Discussion.......................................................93

3.5.1 Characterization of Antibody Attachment........................................................93

3.5.2 Lung Targeting with mAb 201b................................................................97

3.5.3 Brain Targeting with Anti-Synaptophysin and Anti-GFAP................................111

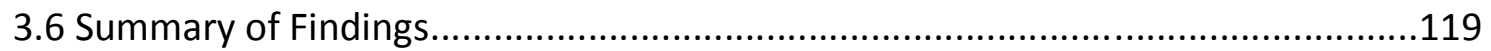

CHAPTER 4: FUTURE DIRECTIONS AND ONGOING WORK.........................................120

4.1 Other Lanthanides as Radiotherapeutics...........................................................120

4.1.1 Nuclear and Chemical Properties of the Lanthanides......................................120

4.1.2 Synthesis and Characterization of $\mathrm{TbPO}_{4}, \mathrm{HoPO}_{4}$, and $\mathrm{LuPO}_{4}$ Nanoparticles...121

4.1.3 Results and Discussion...........................................................................122

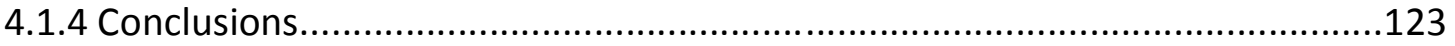

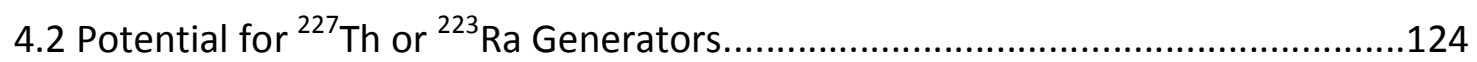

4.2.1 Impetus for ${ }^{227}$ Th or ${ }^{223}$ Ra Generators.........................................................124

4.2.2 Barium Nanoparticles Experimental............................................................125

4.2.3 Barium Nanoparticles Results and Discussion...............................................125

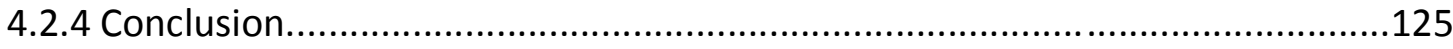

4.3 Future Clinical Applications..............................................................................126

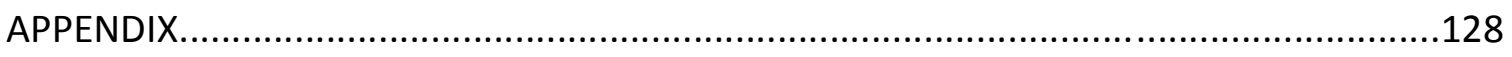

Appendix 1: Total Biodistribution of Starch-Bombesin Gold Nanoparticles...............128 vii 


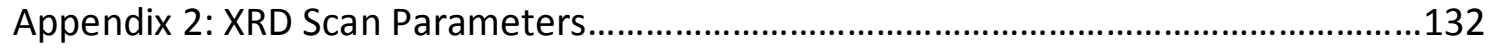

Appendix 3: Antibody:Nanoparticle Ratio Calculation................................................134

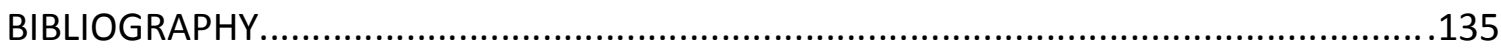

VITA 


\section{LIST OF FIGURES}

Figure 1.5.1 A and B: Simplified decay schemes for ${ }^{227}$ Th and its parent radionuclide, ${ }^{227} \mathrm{Ac}(\mathrm{A})$ and for ${ }^{225} \mathrm{Ac}(\mathrm{B})$ .9

Figure 1.5.2: Purified sample of $1.85 \mathrm{GBq}$ of ${ }^{225} \mathrm{AcCl}_{3}$........ 10

Figure 2.1.1: Simplified decay scheme of ${ }^{64} \mathrm{Cu}$ .18

Figure 2.1.2: Structure of the 14 amino acid bombesin peptide. .20

Figure 2.3.1: Gum arabic coated gold nanoparticles present in SCID mice one month following I.T. injection in PC-3 xenografts measured by NAA. .24

Figure 2.3.2 A and B. Tumor volume (A) and Tumor mass (B) after treatment with gum arabic gold nanoparticles

Figure 2.4.1: Molecular structure of ${ }^{64} \mathrm{Cu}-\mathrm{NO} 2 \mathrm{~A}-$ bombesin conjugate. .28

Figures 2.5.1 A and B: Radio-TLC of starch coated gold nanoparticles before $(A)$ and after (B) filtration .33

Figure 2.5.2: Shift of the plasmon resonance UV-Vis peak of the nanoparticle after starch addition, filtration, and bombesin addition to the surface .34

Figure 2.5.3: Radio-TLC of gum arabic gold nanoparticles.........................................36

Figure 2.5.4: Blocking studies of ${ }^{64} \mathrm{Cu}$-bombesin conjugates using both I.V. and I.P. injection pathways

Figure 2.5.5: Biodistribution of gum arabic coated gold nanoparticles 38

Figure 2.5.5: Biodistribution of starch capped gold nanoparticles 40

Figure 2.6.1: Structure of DTDTPA .44

Figure 2.7.1 A and B: UV-Vis absorption spectra of Au-DTDTPA (A) and Cu-Au-DTDTPA (B).... .50

Figure 2.7.2: TEM showing a characteristic Au-DTDTPA nanoparticle dispersion. 51 ix 
Figure 2.7.3: Copper saturation of Au-DTDTPA as measured by AAS .52

Figure 2.7.4: Stability of Cu-Au-DTDTPA in cysteine .................................................53

Figure 2.7.5 A and B: Stability studies of ${ }^{64} \mathrm{Cu}-\mathrm{Au}$-DTDTPA via radio-TLC.......................54

Figure 2.7.6: Cell viability studies of breast cancer SKBr3 cells................................56

Figures 2.7.7 A and B: Uptake of ${ }^{64} \mathrm{Cu}$-Au-DTDTPA nanoparticles as \%ID/organ (A) and \%ID/g (B) 57

Figure 3.2.1: $\left\{\mathrm{La}_{0.5} \mathrm{Gd}_{0.5}\right\}\left({ }^{225} \mathrm{Ac}\right) \mathrm{PO}_{4} @ 4 \mathrm{GdPO}_{4} @ A u$ nanoparticles after overnight magnetic separation.

Figure 3.2.2: $\left\{\mathrm{La}_{0.5} \mathrm{Gd}_{0.5}\right\}\left({ }^{225} \mathrm{Ac}\right) \mathrm{PO}_{4} @ 4 \mathrm{GdPO}_{4} @$ Au nanoparticles loaded into 10 kDa MWCO membrane for in vitro daughter retention studies. .68

Figure 3.3.1: $\left\{\mathrm{La}_{\mathrm{x}} \mathrm{Gd}_{1-\mathrm{x}}\right\} \mathrm{PO}_{4}$ nanoparticle cores exhibit sizes of $\sim 3-4 \mathrm{~nm}$ 71

Figure 3.3.2 A-D: XRD patterns and Rietveld refinement for $\mathrm{GdPO}_{4}(\mathrm{~A}),\left\{\mathrm{La}_{0.5} \mathrm{Gd}_{0.5}\right\} \mathrm{PO}_{4}$ (B), $\left\{\mathrm{La}_{0.25} \mathrm{Gd}_{0.75}\right\} \mathrm{PO}_{4}(\mathrm{C})$, and $\mathrm{LaPO}_{4}$ (D) .72

Figure 3.3.3: EELS-TEM of a characteristic $\left\{\mathrm{La}_{0.5} \mathrm{Gd}_{0.5}\right\} P \mathrm{PO}_{4} @ 4 \mathrm{GdPO}_{4}$ shell@Au nanoparticle showing the width of the gold surface layer..... 75

Figure 3.3.4: UV-Vis of $\left\{\mathrm{La}_{0.5} \mathrm{Gd}_{0.5}\right\} \mathrm{PO}_{4} @ 4 \mathrm{GdPO}_{4}$ shell@Au nanoparticles......................76

Figure 3.3.5: EELS-TEM confirms the presence of lanthanum, gadolinium, and gold in each nanoparticle.....

Figure 3.3.6: Idealized schematic of a $\left\{\mathrm{La}_{x} \mathrm{Gd}_{1-x}\right\} \mathrm{PO}_{4} @ 4$ LnPO ${ }_{4} @ A u$ nanoparticle .78

Figure 3.3.7: ${ }^{221} \mathrm{Fr}$ retention data for the $\left\{\mathrm{La}_{0.5} \mathrm{Gd}_{0.5}\right\} \mathrm{PO}_{4} @ 4 \mathrm{GdPO}_{4}$ shell@Au and $\left\{\mathrm{La}_{0.25} \mathrm{Gd}_{0.75}\right\} \mathrm{PO}_{4} @ 4 \mathrm{LaPO}_{4}$ shell@Au nanoparticle systems .81

Figure 3.4.1: Structure of lipoamide- $\mathrm{dPEG}_{12}-\mathrm{COOH}$ linker 84

Figure 3.4.2: Amide bond formation by carbodiimide activation .85

Figure 3.5.1: UV-Vis spectra showing the red-shift in the plasmon resonance as PEG and antibody are added to citrate coated $\left\{\mathrm{La}_{0.5} \mathrm{Gd}_{0.5}\right\} \mathrm{PO}_{4} @ 4 \mathrm{GdPO}{ }_{4} @ \mathrm{Au}$ nanoparticles......94 
Figure 3.5.2: Competition assay of

Figure 3.5.3: Biodistribution of $\left\{\mathrm{La}_{0.5} \mathrm{Gd}_{0.5}\right\}\left({ }^{225} \mathrm{Ac}\right) \mathrm{PO}_{4} @ 4 \mathrm{GdPO}_{4}$ shell@Au nanoparticles with various antibody loading.

Figure 3.5.4: TEM of $\left\{\mathrm{Gd}_{0.5} \mathrm{La}_{0.5}\right\} \mathrm{PO}_{4} @ 4 \mathrm{GdPO}_{4}$ shell@Au-mAb 201b nanoparticles.....100

Figure 3.5.5 A and B: Biodistribution of $\left\{\mathrm{Gd}_{0.5} \mathrm{La}_{0.5}\right\} \mathrm{PO}_{4} @ 4 \mathrm{GdPO}_{4}$ shell@Au-PEG-mAb 201b nanoparticles at 1 hour post-injection (A) and 24 hours post-injection (B).......101

Figure 3.5.6: CT-SPECT scans of $\left\{\mathrm{La}_{0.5} \mathrm{Gd}_{0.5}\right\}\left({ }^{225} \mathrm{Ac}\right) \mathrm{PO}_{4} @ 4 \mathrm{GdPO}_{4}$ shell@Au-mAb-201b nanoparticles....

Figure 3.5.7 A-D: Biodistribution and lung uptake of $\left\{\mathrm{La}_{0.5} \mathrm{Gd}_{0.5}\right\}\left({ }^{225} \mathrm{Ac}\right) \mathrm{PO}_{4} @ 4 \mathrm{GdPO}_{4}$ shell@Au-mAb-201b nanoparticles with and without clodronate liposomes. 106

Figure 3.5.8: Slides showing EMT-6 tumor colonies in lung tissue

Figure 3.5.9: Lung tissue showing EMT-6 tumor colony staining .110

Figure 3.5.10: Immunohistochemistry of brain tissue with anti-synaptophysin labeled nanoparticles.

Figure 3.5.11: Immunohistochemistry of brain tissue with anti-GFAP labeled nanoparticles

Figure 3.5.12: Catheterization technique for I.V. injection.

Figure 3.5.13: $\left\{\mathrm{Gd}_{0.5} \mathrm{La}_{0.5}\right\} \mathrm{PO}_{4} @ 4 \mathrm{GdPO}_{4}$ shell@Au nanoparticles labeled with antisynaptophysin bind receptors in CNS synapses.

Figure 3.5.14: $\left\{\mathrm{Gd}_{0.5} \mathrm{La}_{0.5}\right\} \mathrm{PO}_{4} @ 4 \mathrm{GdPO}_{4}$ shell@Au nanoparticles labeled with anti-GFAP bind receptors in astrocytes. .118

Figure 4.1.1: XRD pattern of $\mathrm{LuPO}_{4}$ 


\section{LIST OF TABLES}

Table 1.5.1: Range and kinetic energies of ${ }^{225} \mathrm{Ac}$ daughter products.................................14

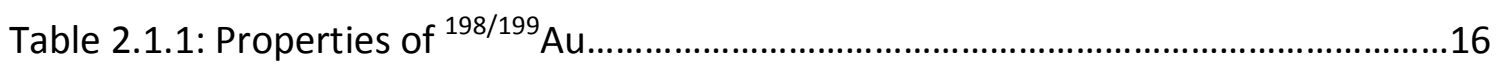

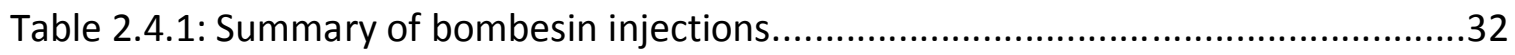

Table 2.5.1: Activity in each wash of bombesin coated gold nanoparticles...................34

Table 2.7.1: DLS properties of Au-DTDTPA and Cu-Au-DTDTPA................................51

Table 3.3.1: Nanoparticle growth of $\left\{\mathrm{La}_{0.5} \mathrm{Gd}_{0.5}\right\} \mathrm{PO}_{4} @ 4 \mathrm{GdPO}_{4}$ shell@Au......................73

Table 3.3.2: Retention of ${ }^{225} \mathrm{Ac}$ and its daughter ${ }^{221} \mathrm{Fr}$ in different

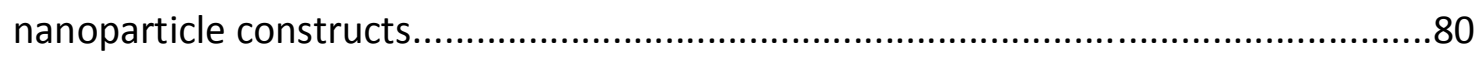

Table 3.5.1: Hydrodynamic diameter and zeta potential of $\left\{\mathrm{La}_{0.5} \mathrm{Gd}_{0.5}\right\} \mathrm{PO}_{4} @ \mathrm{GdPO}_{4} @ A u-\mathrm{mAb}-201 \mathrm{~b}$ nanoparticles. .94

Table 3.5.2: Labeling efficiency of mAb $201 \mathrm{~b}$ onto $0.5 \mathrm{mg}$ of $\left\{\mathrm{La}_{0.5} \mathrm{Gd}_{0.5}\right\}\left({ }^{225} \mathrm{Ac}\right) \mathrm{PO}_{4} @ 4 \mathrm{GdPO}_{4}$ shell@Au nanoparticles. 97

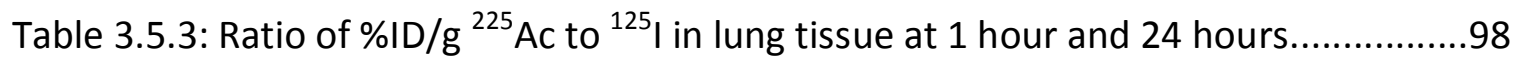




\title{
LIST OF ABBREVIATIONS
}

\author{
Abbreviation \\ Definition \\ MRI \\ Magnetic resonance imaging \\ CT \\ computed tomography \\ LET \\ DNA \\ Linear energy transfer \\ EPR \\ Deoxyribonucleic acid \\ DMPS \\ DMSA \\ Enhanced permeability and retention \\ 2,3-dimercapto-1-propanesulfonic acid \\ Dimercaptosuccinic acid \\ SRIM/TRIM \\ Stopping range of ions in matter/transport of ions in \\ matter \\ SPECT \\ PET \\ TETA \\ acid \\ CB \\ IT \\ GRP \\ DTPA \\ ACS \\ THPAL \\ DPBS \\ UV-Vis \\ ICRSCID \\ immunodeficient \\ NAA \\ TEM \\ DLS \\ ID \\ RES \\ MURR \\ NO2A \\ BSA \\ TLC \\ IV \\ IP \\ PEG \\ Single photon emission computed tomography \\ Positron emission tomography \\ 1,4,8,11-tetraazacyclotetradecane-1,4,8,11-tetraacetic \\ Cross-bridged \\ Intratumoral \\ Gastrin-releasing peptide \\ diethylene triamine pentacetic acid \\ American Chemical Society \\ Tris-hydroxylmethyl phosphine alanine \\ Dulbecco's phosphate buffered saline \\ Ultraviolet-visible \\ Imprinting control region severe combined \\ Neutron activation analysis \\ Transmission electron microscopy \\ Dynamic light scattering \\ Injected dose \\ Reticuloendothelial system \\ University of Missouri Research Reactor \\ 1,4,7-Triazacyclononane-1,4-diacetic acid \\ Bovine serum albumin \\ Thin layer chromatography \\ Intravenous \\ Intraperitoneal \\ Polyethylene glycol \\ 1-Ethyl-3-(3-dimethylaminopropyl)carbodiimide)




$\begin{array}{ll}\text { NHS } & \text { N-hydroxy succinimide } \\ \text { AAS } & \text { Atomic absorption spectroscopy } \\ \text { DTDTPA } & \text { Dithiolated diethylene triamine pentacetic acid } \\ \text { BA } & \text { Bis-anhydride } \\ \text { DMF } & \text { Dimethyl formamide } \\ \text { NMR } & \text { Nuclear magnetic resonance } \\ \text { DI } & \text { Deionized } \\ \text { RSA } & \text { Rat serum albumin } \\ \text { MTT } & \text { 3-(4,5-dimethylthiazol-2-yl)-2,5-diphenyltetrazolium } \\ \text { bromide } & \\ \text { MWCO } & \text { Molecular weight cutoff } \\ \text { XRD } & \text { X-ray diffraction } \\ \text { EELS } & \text { Electron energy loss spectroscopy } \\ \text { TPP } & \text { Tripolyphosphate } \\ \text { mAb } & \text { Monoclonal antibody } \\ \text { GFAP } & \text { Glial fibrillary acidic protein } \\ \text { TCEP } & \text { Tris(2-carboxyethyl)phosphine } \\ \text { hEGFR } & \text { Human epidermal growth factor receptor } \\ \text { DAB } & \text { Diaminobenzadine } \\ \text { CNS } & \text { Central nervous system }\end{array}$




\begin{abstract}
Several different investigations are discussed in this dissertation, with the common thread of using gold-containing nanomaterials in nuclear medicine applications. Gold nanoparticles form a non-toxic surface layer that can be easily functionalized with biological targeting agents. The first set of experiments focuses on attempts to develop cancer therapeutic and imaging agents using the radionuclides ${ }^{198 / 199} \mathrm{Au}$. Gold nanoparticles with both passive (gum arabic coated) and active (bombesin) targeting are examined. Additionally, the possibility of attaching a chelate to bind the PET imaging radionuclide ${ }^{64} \mathrm{Cu}$ with DTDTPA is explored.
\end{abstract}

The second area of emphasis focuses on developing a nanoparticle capable of retaining the radioactive decay daughters of the in vivo $\alpha$ generator ${ }^{225} \mathrm{Ac}$. This generator system produces 4 successive $\alpha$ emissions in its decay chain. However, daughter products escape and cause renal toxicity. A $\mathrm{LnPO}_{4}$ nanoparticle coated with gold can both retain the energetic daughter products in the decay chain and be easily functionalized to deliver radiation to biologically relevant targets in vivo.

Nanoparticle technology allows for the combination of multiple therapeutic and imaging modalities by a single platform. Unlike conventional approaches, the multiatom nature of nanoparticles allows for delivery of large, varied payloads to biological receptors. The nanomaterials discussed here can theoretically be used for any combination of PET/SPECT imaging, MRI contrast, or $\alpha / \beta^{-}$radiotherapy. 


\section{CHAPTER 1:}

\section{INTRODUCTION TO NANOPARTICLE BASED RADIATION THERAPY}

\subsection{Cancer Epidemiology}

Great strides have been made in the diagnosis and treatment of a wide variety of cancers. Relative survival rates of all cases have risen to $67 \%$ for cancers diagnosed between 2001 and 2007, compared with only 45\% for cancers diagnosed between 1975 and 1977. Despite this progress, cancer remains the second leading cause of death in the United States, accounting for nearly one in every four deaths. ${ }^{1}$ Globally, cancer incidence is expected to rise $75 \%$ by $2030 .^{2}$ Early diagnosis and detection of cancer increases the success of therapeutic regimens. Unfortunately, many types of cancer will metastasize, creating tumors in distant parts of the body prior to diagnosis. Neoplastic cells can lie dormant for decades before developing into tumors. ${ }^{3}$ The presence of metastatic disease drastically decreases survival. For example, primary breast cancer tumors are currently cured with a $98.6 \%$ success rate, as measured by the relative 5 year survival fraction. Regional spread of tumor cells via the lymphatic system lowers survival expectancy to $83.8 \%$. In contrast, only $23.3 \%$ of breast cancer patients with distant, metastatic spread of cancer survive five years after diagnosis. ${ }^{4}$ 


\subsection{Current Oncology Therapies}

Current cancer treatment falls into three basic categories: surgery, chemotherapy, and radiotherapy. Most patients diagnosed with cancer undergo some sort of surgery to remove large, primary tumors. When the tumor is localized, surgical resection effectively treats a wide variety of tumors. Unfortunately, a number of factors can render a tumor inoperable. The tumor may be connected to or embedded within a vital organ such as the heart or brain or the tumor may have metastasized to distant sites, rendering removal of the original tumor ineffectual. Finally, the tumor may not have adequate margins of neighboring healthy tissue to be safely removed. Even a small remaining neoplastic fraction will quickly regenerate the removed tumor mass. Surgery represents an effective, if invasive, treatment for isolated solid primary tumors.

Inoperable tumors, metastases, and dormant circulating tumors require other methods of treatment. Chemotherapy, the use of cytotoxic chemicals to attack neoplastic cells, can be effective as a standalone therapy or in combination with surgery and/or radiation to prevent the spread and recurrence of surgically removed neoplasms. ${ }^{5}$ Most chemotherapeutic agents act non-specifically on rapidly dividing cells which include tumor cells, bone marrow, intestinal cells, and hair follicles. The effect of chemotherapeutics on healthy tissues causes numerous side effects associated with cancer treatment, including decreased immune function and anemia from bone marrow destruction, nausea and vomiting from intestinal cell toxicity, and alopecia (hair loss) from the loss of hair follicles. ${ }^{6,7,8}$ 
Radiation therapy is a method often used either as a standalone treatment or in combination with surgery and/or chemotherapy. The two predominant types of radiotherapy currently in clinical use are external beam radiotherapy and brachytherapy. External beam radiotherapy uses either x-rays, protons, or $\alpha$ particles to destroy the target cancer cells, often guided by advanced imaging techniques such as magnetic resonance imaging (MRI) and computed tomagraphy (CT). ${ }^{9}$ However, external beam radiotherapy disrupts healthy tissue as it traverses the path to the tumor, causing harmful side effects. Brachytherapy uses implantable "seeds" containing a radioactive isotope. These seeds are placed in the immediate vicinity of the tumor and deliver a continual dose until the source is removed or decays. Implantable brachytherapy seeds are particularly common and effective in treatment of inoperable prostate cancer. ${ }^{10}$ Brachytherapy seeds also have the potential to shift in vivo, delivering dose to healthy tissue or causing a radiological hazard if excreted from the body. Large doses to normal tissue result in undesirable side effects such as incontinence and impotence. ${ }^{11}$

\subsection{Targeted Radiotherapies and Types of Therapeutic Radiation}

In order to simultaneously improve cancer patient outcomes and quality of life, more specific therapeutic modalities must be pursued. Side effects would be drastically reduced, if not eliminated, by development of radiotherapeutic compounds that spare healthy tissue while delivering cytotoxic doses to tumor cells. Currently, there are two targeted radiotherapy compounds approved by the FDA for human use. BEXXAR utilizes

the $\beta^{-}$particle emitted from ${ }^{131}$ I in treatment of follicular lymphoma while Zevalin 
incorporates the $\beta^{-}$emitting radionuclide ${ }^{90} Y$ for treatment of non-Hodgkin's Iymphoma. ${ }^{12,13}$

There are two distinct types of radiation used for therapeutic purposes.

Photons, $\beta^{-}$particles, and Auger electrons deliver low linear energy transfer (LET) radiation while protons and $\alpha$ particles deliver high LET radiation. Low LET and high LET radiation differ drastically in their properties and mechanism of action and therefore their clinical applicability. Both types of radiation destroy cancer cells by inducing double strand breaks in cellular deoxyribonucleic acid (DNA). This type of lesion proves difficult for the cell to repair and leads to fatal chromosomal aberrations. ${ }^{14}$ Low LET radiation acts primarily by generating reactive oxygen species as a result of ionization events. Because low LET radiation acts, at least in part, via these intermediaries, their efficacy can be decreased based on external factors such as cell oxygenation or cell cycle considerations. ${ }^{15,16}$ Cells which are hypoxic or anoxic require 2-3 times more activity of low LET radiation to produce the same level of toxicity. ${ }^{17}$ Dividing cells are able to repair DNA damage, including double strand breaks, during the $S$ phase of mitosis when chromosomes are being duplicated. They are least likely to successfully repair DNA lesions during the mitotic, or $\mathrm{M}$ phase. ${ }^{15}$

In contrast, high LET radiation creates double strand breaks by acting directly on DNA. As a result, the effects of high LET radiation depend less on external factors. The path length between ionization events closely approximates the distance between cross-sectional base pairs in the DNA molecule. ${ }^{15}$ Only a few traversals of the nucleus is 
required to generate a cell kill as opposed to the thousands necessary in $\beta^{-}$therapy. ${ }^{18}$ Thus, $\alpha$ and proton therapy can cause tumor cytotoxicity with only a fraction of the activity required in $\beta^{-}$therapy, i.e. $\alpha$ therapy has a much higher relative biological effectiveness. Because high LET radiation deposits its energy in a small, localized space, it limits collateral damage to healthy tissue. Unfortunately, this effect also limits the scope of $\alpha$ therapy to small micrometastases, disseminated/blood-borne neoplasms, and film-like peritoneal tumors. ${ }^{19}$ Crossfire effects from the longer range of $\beta^{-}$particles provide a better option for treating large, solid tumors which cannot be surgically resected or treated with chemotherapy/external beam radiotherapy. ${ }^{20}$

The bifunctional chelate approach represents a major paradigm for

radiopharmaceutical research in both $\alpha$ and $\beta^{-}$therapy. ${ }^{21}$ In this approach, a radiometal is stably sequestered by a chelating agent bound to a targeting molecule such as a peptide or antibody. The chelate must be able to bind the radiometal quickly relative to the radionuclide half-life in order to maximize radiochemical yield. It also must form a very stable bond (both thermodynamically and kinetically) that will remain impervious to attack by the various proteins and salt concentrations found in the biological environment.

\subsection{Nanoparticles for Cancer Therapy}

Recently, nanoparticles have emerged as an exciting alternative to molecular chelating agents for delivering radioisotopes in cancer therapy. The wide swath of 
constructs that falls under the umbrella of nanoparticles possess a number of exciting properties for delivery of both radioactive and non-radioactive therapeutic agents. Nanoparticles possess extraordinary versatility, where the same platform can be radically changed by modifying size, shape, and surface characteristics of the particle. These changes alter the pharmacokinetic profile of the nanoparticle. In vivo characteristics such as biodistribution, excretion pathway, and drug release timing can all be tuned by slight changes in particle composition. ${ }^{22}$

Many important processes in the body are regulated by receptors which are present in very low concentrations, yet have a very high affinity for their associated substrate. These low receptor concentrations make them difficult to target for therapeutic and imaging purposes. ${ }^{23}$ Using a nanoparticle rather than a molecular bifunctional chelate can drastically increase the dose delivered to each receptor site. While a traditional molecular chelate typically binds one radiometal, a single nanoparticle can deliver multiple radioactive atoms to a single receptor site. Additionally, the size of the nanoparticle makes it possible to link multiple targeting vectors onto a single particle. Emerging data on tumor heterogeneity suggests that variations between tumor cells even within a single tumor type may frustrate attempts to effectively target all of the tumor cells. Incorporating multiple targeting mechanisms onto each particle holds the potential to broaden the effect of targeted radiopharmaceuticals. $^{24}$ 
Nanoparticles possess some degree of innate targeting towards tumors in vivo.

The remarkable growth rate of tumors requires recruitment of neovasculature to provide sufficient nutrients to the neoplasm. Tumor cells often release large amounts of vascular epithelial growth factor to promote angiogenesis and vascular permeability. As a result of the increased permeability, large constructs such as nanoparticles and liposomes are filtered from the bloodstream into the interstitial space surrounding the tumor. In normal tissue, these species are typically removed from the interstitial space by the lymphatic system. However, in tumor tissue the lymphatic system is largely disabled, increasing the residence time of the nanoparticle in the interstitial space. This combination of leaky, permeable vasculature and disabled lymphatic function is known as the enhanced permeability and retention (EPR) effect. ${ }^{25}$

The increased size of nanoparticle constructs relative to bifunctional chelate platforms presents unique challenges. While increased passive tumor targeting occurs via the EPR effect, specific targeting is hindered. Large nanoparticles have difficulty extravasating out of the vascular space, potentially limiting application to tumors with vascular targets, blood-borne neoplasms, and peritoneal cancers. Various techniques such as mild hyperthermia can improve nanoparticle extravasation on clinically relevant time scales. $^{26}$

Much of the work on nanotechnology has focused on gold nanostructures. While gold colloids were known and used in medieval times as a stain, monodisperse gold nanoparticles were first routinely synthesized in the modern era by Turkevich, et al. 
in $1951 .^{27}$ Their procedure produces spherical gold nanoparticles of diameters from 10$20 \mathrm{~nm}$ in aqueous solution. Ionic gold $\left(\mathrm{Au}^{+3}\right)$ is reduced by citrate with heat to neutral gold. Brust later pioneered an organic phase gold synthesis and solvent transfer method by modifying the surface groups on the nanoparticle. This method involves reduction of ionic gold by sodium borohydride in toluene with tetraocytlammonium bromide capping agent. $^{28}$ Due to its relative inertness and facile functionalization, gold makes an attractive surface coating for other nanoparticle materials. For instance, the surface plasmon resonance of gold provides a visual marker for $\mathrm{MRI}$ active, gold coated $\mathrm{Fe}_{3} \mathrm{O}_{4}$ particles. $^{29}$

\subsection{In vivo $\alpha$ generator therapy}

Alpha generator therapies use radionuclides with four $\left({ }^{223} \mathrm{Ra},{ }^{225} \mathrm{Ac}\right)$ or five $\left({ }^{227} \mathrm{Th}\right)$ $\alpha$ particles in their decay chain. Decay chains for ${ }^{227} \mathrm{Th}$ and ${ }^{225} \mathrm{Ac}$ are shown in Figures $1 a^{30}$ and $1 b$. Currently there are two barriers to development of clinically relevant in vivo $\alpha$ generators. The first challenge consists of producing a reliable and sufficient supply of the radionuclides. Secondly, a means of sequestering the radioactive daughter products in vivo must be devised to prevent toxicity to non-target tissue. 
A

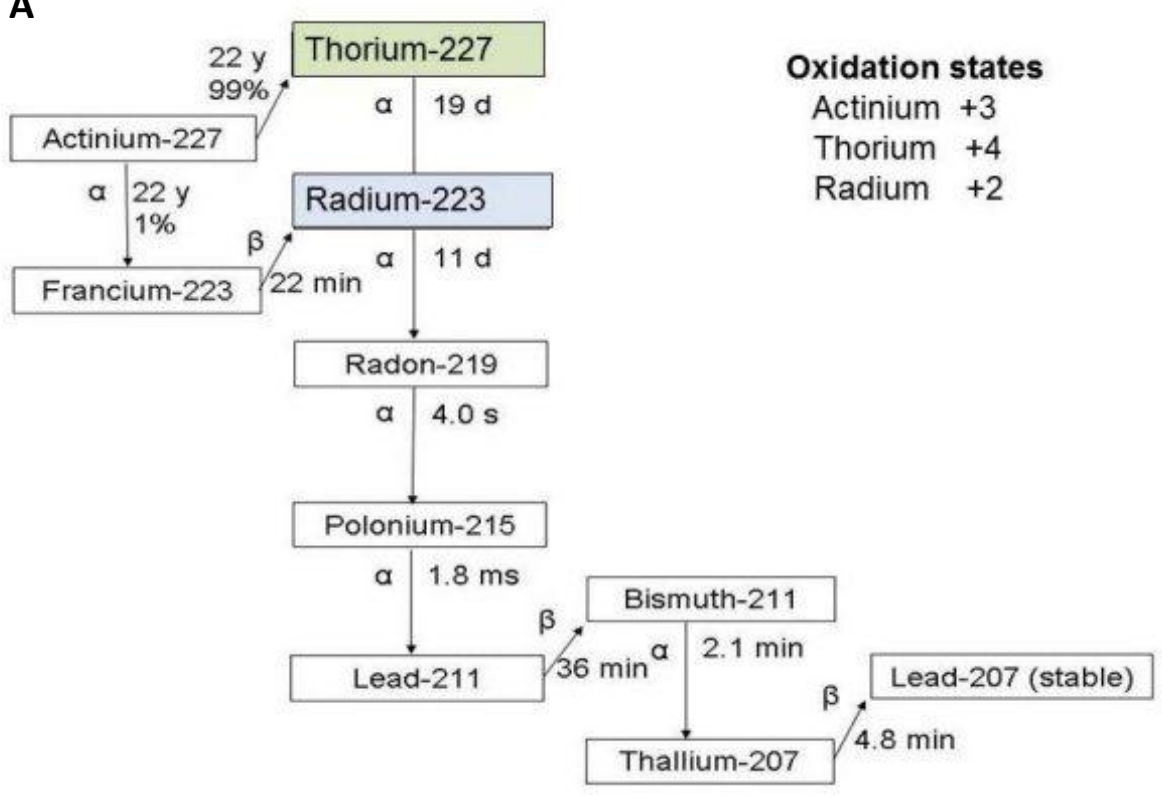

B

$225 \mathrm{Ac}$

$10.0 \mathrm{~d}$

$\downarrow \alpha$

${ }^{221} \mathrm{Fr}$

$4.9 \mathrm{~m}$

$\alpha$

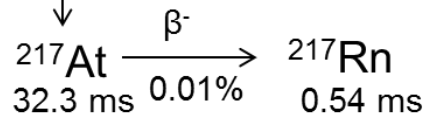
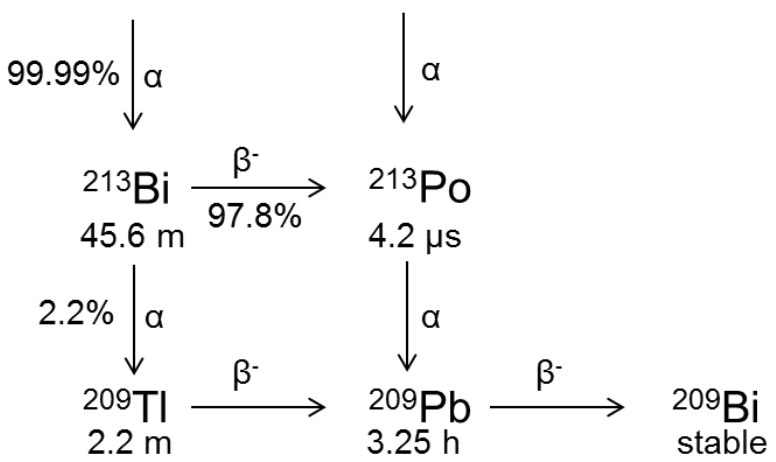

Figure 1.5.1 A and B: Simplified decay schemes for ${ }^{227} \mathrm{Th}$ and its parent radionuclide,

${ }^{227} \mathrm{Ac}(\mathrm{A})$ and for ${ }^{225} \mathrm{Ac}(\mathrm{B})$. 
Currently, ${ }^{225} \mathrm{Ac}$ is available from a ${ }^{229} \mathrm{Th}$ generator produced at Oak Ridge National Laboratory. ${ }^{31}$ The ${ }^{229} \mathrm{Th}$ comes from legacy stockpiles of ${ }^{233} \mathrm{U}$ developed during the cold war era and produces no carrier added ${ }^{225} \mathrm{Ac}$. Alternative strategies for production of ${ }^{225} \mathrm{Ac}$ include using stockpiled ${ }^{226} \mathrm{Ra}$ seeds in a ${ }^{226} \mathrm{Ra}(\mathrm{p}, 2 \mathrm{n}){ }^{225} \mathrm{Ac}$ reaction. This reaction has been used with proton energies of $16.8 \mathrm{MeV}$ on a $0.0125 \mathrm{mg}$ target for 7 hours with a current of $10 \mu \mathrm{A}$ to produce $78 \mathrm{kBq}$ of ${ }^{225} \mathrm{Ac} .{ }^{32}$ This method also produces ${ }^{224} \mathrm{Ac}$, which is decayed away before separation and ${ }^{226} \mathrm{Ac}$, which has a negligible yield at 16.8 MeV. A third method for production of ${ }^{225} \mathrm{Ac}$ involves the spallation reaction on ${ }^{232} \mathrm{Th}$. Cross section data obtained at $800 \mathrm{MeV}$ proton energy indicate that 10 day irradiations at the Los Alamos Neutron Science Center facility will be capable of producing $740 \mathrm{GBq}$ of ${ }^{225} \mathrm{Ac}$. A non-negligible amount of ${ }^{227} \mathrm{Ac}(0.25 \%)$ will also be produced. An additional $13 \mathrm{GBq}$ of no carrier added ${ }^{225} \mathrm{Ac}$ could be made available from simultaneous production of the ${ }^{225}$ Ra parent. ${ }^{33}$ Further studies are required to determine if the ${ }^{227} \mathrm{Ac}$ impurity is sufficient to cause long term radiation damage in vivo.

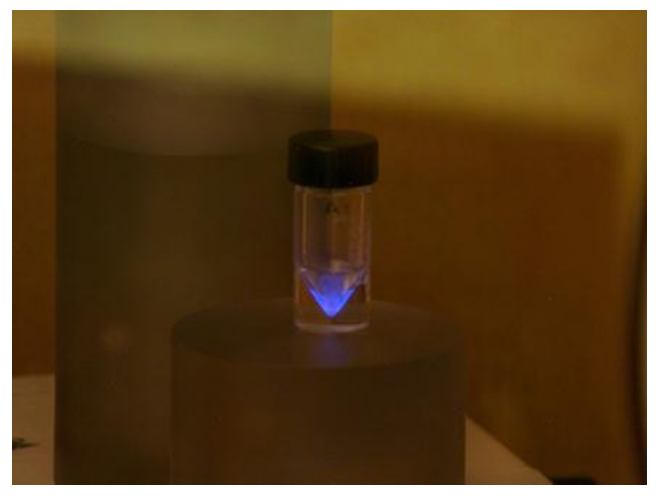

Figure 1.5.2: Purified sample of $1.85 \mathrm{GBq}$ of ${ }^{225} \mathrm{AcCl}_{3}$. Alpha particles from the sample cause the glass vial to radioluminesce. 
Thorium-227 and ${ }^{223}$ Ra are primarily available in small quantities as decay products of ${ }^{235} \mathrm{U}$ and ${ }^{227} \mathrm{Ac}$. Both the United States and Europe possess modest stockpiles of ${ }^{227} \mathrm{Ac}$. The ${ }^{227} \mathrm{Ac}$ parent can also be produced with good yield from the ${ }^{226} \mathrm{Ra}(\mathrm{n}, \mathrm{\gamma}){ }^{227} \mathrm{Ac}$ reaction. ${ }^{34}$ Successive neutron capture on ${ }^{227} \mathrm{Th}$ can produce ${ }^{228} \mathrm{Th}$ and ${ }^{229} \mathrm{Th}$, the parent isotope for ${ }^{225} \mathrm{Ac}^{35}$

Beyond radionuclide availability, the emission of multiple $\alpha$ particles presents unique challenges for specific delivery of therapeutic radiation. Namely, the chemical behavior of daughter radionuclides often differs radically from that of the parent radionuclide. For example, the bivalent ${ }^{223}$ Ra decays to the unreactive noble gas radon while the trivalent ${ }^{225} \mathrm{Ac}$ decays to the monovalent francium. Chemical behavior along the decay chain of in vivo generators switches from cationic to anionic and back again, frustrating attempts to contain the radionuclides within a single chelate. The recoil energy of the daughter radionuclides presents an equally daunting challenge. Each $\alpha$ particle emission creates a recoil energy of $>100 \mathrm{keV}$ (four orders of magnitude greater than the energy of a typical covalent bond) on the daughter radionuclide. As a result, daughters chelated by traditional molecular species will not remain bound to the bioconjugate at the targeting site. ${ }^{36}$

The in vivo $\alpha$ generator ${ }^{223}$ Ra provides an example of an effective targeted $\alpha$ therapy generator for treatment of metastatic bone cancer. ${ }^{37}$ Radium-223 chloride (Alpharadin) has been granted Fast Track designation by the U.S. Food and Drug Administration for the treatment of hormone-refractory prostate cancer in patients with 
bone metastases. ${ }^{38}$ It is effective because radium mimics calcium in vivo, providing a high affinity for bone. Further, the daughter products either have short half-lives $(<5$ minutes) or have a high affinity for bone $\left({ }^{211} \mathrm{~Pb}, \mathrm{t}_{1 / 2}=36 \mathrm{~m}\right)$. Translation of in vivo $\alpha$ generators to anything besides metastatic bone cancer with a calcium mimic will require a different mechanism for both delivering and retaining the radioactive daughters in the target tissue.

Other methods under investigation for sequestration of $\alpha$ emitting daughters involve chelation with DOTA or analogous macrocyclic chelators and incorporation into liposomes/polymerosomes, fullerenes, or nanocrystals. In the case of ${ }^{225} \mathrm{Ac}$ based therapeutics, ${ }^{213} \mathrm{Bi}$ daughter migration to the kidney represents the dose limiting factor. Scavengers such as 2,3-dimercapto-1-propanesulfonic acid (DMPS) and dimercaptosuccinic acid (DMSA) or non-radioactive Bi competition mitigate, but do not effectively eliminate this limitation. ${ }^{39}$

Another challenge is that labeling efficiency for molecular chelates depends on the ratio of chelate to ${ }^{225} \mathrm{Ac}$. Examples from literature studies typically exhibit either high synthetic yield or high specific activity, but not both. In order to achieve high synthetic yield (>97\%), Essler et al. use huge excesses of chelate/peptide conjugates $\left(6,700\right.$ conjugates per ${ }^{225} \mathrm{Ac}$ atom). The resulting low specific activity ( $\left.72 \mathrm{MBq} / \mu \mathrm{mol}\right)$ would be suitable only for receptors that are in high abundance in vivo. ${ }^{40}$ Effectively targeting receptors with low in vivo concentrations requires high specific activity. Song et al. produce higher specific activity bioconjugates $(4.44 \mathrm{GBq} / \mu \mathrm{mol})$ but at the expense 
of labeling efficiency (12.0\%). ${ }^{41}$ Given the limited supply of ${ }^{225} \mathrm{Ac}$ and the importance of receptors with low concentrations, development of a high yield, high specific activity synthesis is paramount.

Single and multi-layer liposomes are under evaluation for their ability to sequester ${ }^{225} \mathrm{Ac}$ and its $\alpha$-emitting daughters. Unfortunately, preparations of single wall liposomes suffer from both low ${ }^{225} \mathrm{Ac}$ incorporation (6.4\%) and poor daughter retention $\left({ }^{213} \mathrm{Bi}<10 \%\right) .{ }^{42}$ Further, single wall liposomes struggle to quantitatively retain the ${ }^{225} \mathrm{Ac}$ parent radionuclide. Despite the lofty sequestration predictions of theoretical models, experimental retention of ${ }^{225} \mathrm{Ac}$ and ${ }^{213} \mathrm{Bi}$ appears much lower. Active loading of liposomes increases ${ }^{225} \mathrm{Ac}$ incorporation to $73 \%$, though ${ }^{225} \mathrm{Ac}$ retention only reaches $81 \%{ }^{43}$ Despite the higher synthetic yield, ${ }^{213} \mathrm{Bi}$ retention does not improve. Recent theoretical work postulates ${ }^{213} \mathrm{Bi}$ retention as high as $80 \%$ in multi-walled polymerosomes. The proposed polymerosomes require a large bulk $(800 \mathrm{~nm}$ in diameter) to achieve this level of retention. ${ }^{44}$ Such large particles would not extravasate out of the vasculature and would not capitalize on the enhanced permeability and retention effect. ${ }^{45,46} \mathrm{~A}$ final strategy for ${ }^{225} \mathrm{Ac}$ sequestration involves incorporation into fullerenes. This method exhibits a low synthetic yield (1\%) and little to no ${ }^{221} \mathrm{Fr}$ daughter sequestration. $^{47}$

A final method for retaining ${ }^{225} \mathrm{Ac}$ and its $\alpha$-emitting daughter radionuclides directly incorporates Ac into the crystal structure of a nanoparticle. ${ }^{48,49}$ Due to isomorphism between Ac and the lanthanides, Ac co-crystallizes with lanthanide 
phosphates. Both Ac and the lanthanides (with the exception of cerium) almost exclusively form +3 cations. Additionally, the light lanthanides are large enough to approximate the ionic radius of Ac. Incorporating Gd into the crystal provides a sufficient paramagnetic moment for separation of the nanoparticles with a NdFeB magnet. ${ }^{49}$ Addition of a Au surface layer facilitates surface modification, prevents Gd leakage in vitro, and prevents in vivo reactivity. Ranges of recoiling ${ }^{225} \mathrm{Ac}$ daughters in gold calculated by the stopping range of ions in matter/transport of ions in matter (SRIM/TRIM) program are shown below in Table 1.5.1. ${ }^{50}$

Table 1.5.1: Range and kinetic energies of ${ }^{225} \mathrm{Ac}$ daughter products

\begin{tabular}{|l|l|l|l|}
\hline Daughter & Decay energy (MeV) & Daughter energy $(\mathrm{keV})$ & Range in gold $(\mathrm{nm})$ \\
\hline${ }^{221} \mathrm{Fr}$ & 6.3 & 112 & 11.3 \\
\hline${ }^{217} \mathrm{At}$ & 7.1 & 129 & 12.5 \\
\hline${ }^{213} \mathrm{Bi}$ & 5.9 & 109 & 11.3 \\
\hline${ }^{209} \mathrm{~Pb}$ & 8.4 & 158 & 12.0 \\
\hline
\end{tabular}

In addition to Alpharadin, there are two other current clinical trials of in vivo $\alpha$ generators involving ${ }^{225} \mathrm{Ac}$. The first phase I study is currently recruiting patients at Memorial Sloan Kettering Cancer Center in New York. This clinical trial utilizes the humanized antibody HuM195 against the CD33 protein, found in acute myeloid leukemia. In the study, ${ }^{225} \mathrm{Ac}$ is chelated to DOTA, which is then bound to HuM195. ${ }^{51}$ The second study, organized by M.D. Anderson Cancer Center, uses the antibody lintuzumab for treatment of acute myeloid leukemia. ${ }^{52}$ 


\title{
CHAPTER 2:
}

\section{GOLD NANOPARTICLES FOR THERAPY OF UNRESECTABLE}

\author{
PROSTATE CANCER
}

\subsection{Introduction}

\subsubsection{Production, Separation, and Nuclear Properties of Radiogold}

Prostate cancer is the most common form of cancer in males, with 241,000 new cases estimated to be diagnosed in 2012.1 Many of these tumors are not eligible for surgical resection. Methods such as radiotherapy/brachytherapy successfully treat these neoplasms or shrink them to the point where they can be surgically resected.

Radiogold possesses favorable properties for $\beta^{-}$therapy of tumors. Production of radiogold occurs via neutron irradiation. Only one stable isotope of gold, ${ }^{197} \mathrm{Au}$, occurs in nature, making enrichment unnecessary. Neutron absorption by natural gold produces a large amount of ${ }^{198} \mathrm{Au}$ and a smaller fraction of ${ }^{199} \mathrm{Au}$. A summary of their nuclear properties is shown below in Table 2.1.1.

Both gold radionuclides have approximately a 3 day half-life and release a $\beta^{-}$ particle. Lower ratios of ${ }^{198} \mathrm{Au} /{ }^{199} \mathrm{Au}$ (higher production of ${ }^{199} \mathrm{Au}$ ) are produced using a 
Table 2.1.1: Properties of ${ }^{198 / 199} \mathrm{Au}^{53,54}$

\begin{tabular}{|l|l|l|l|l|l|}
\hline Radionuclide & Half-Life (d) & $\beta^{-}$energy (keV) & $\gamma$ energy (keV) & $\sigma_{\text {thermal }}(\mathrm{b})$ & $\sigma_{\text {epithermal }}(\mathrm{b})$ \\
\hline${ }^{197} \mathrm{Au}$ & Stable & & & 1550 & 98.7 \\
\hline${ }^{198} \mathrm{Au}$ & 2.70 & 719 & $412(96 \%)$ & 26,000 & \\
\hline${ }^{199} \mathrm{Au}$ & 3.14 & 453 & $158(40 \%)$ & & \\
\hline
\end{tabular}

high thermal/epithermal flux and long irradiation times. The high energy $\gamma$ ray at 411.8 keV, which occurs with $96 \%$ abundance in ${ }^{198} \mathrm{Au}$, presents a major drawback for radiotherapy purposes. ${ }^{54}$ This $y$ ray delivers a whole body radiation dose to healthy tissue in the patient. In contrast, ${ }^{199} \mathrm{Au}$ has an ideal imaging $\gamma$ ray of $158 \mathrm{keV}(40 \%$ abundance). ${ }^{54}$ Current single photon emission computed tomography (SPECT) equipment designed to image the $140 \mathrm{keV} \gamma$ ray from ${ }^{99 \mathrm{~m}} \mathrm{Tc}$ will easily detect the ${ }^{199} \mathrm{Au} \gamma$ with little or no modification. Monte Carlo N-Particle analysis of these two $\gamma$ rays in a standard reference man indicates that the ${ }^{198} \mathrm{Au} \gamma$ gives a whole body dose 2.37 times higher than the ${ }^{199} \mathrm{Au} y \cdot{ }^{55}$ The non-specific, whole body dose can disrupt normal function and lead to carcinogenesis in other parts of the body.

No carrier added ${ }^{199} \mathrm{Au}$, which will provide a lower whole body $\gamma$ dose, can be produced by neutron irradiation of ${ }^{198} \mathrm{Pt}\left(7.16 \%\right.$ natural abundance) ${ }^{53}$ The ${ }^{198} \mathrm{Pt}(\mathrm{n}, \mathrm{\gamma}){ }^{199} \mathrm{Pt}$ reaction has a high cross section (3.8 barn thermal, 56 barn epithermal) and the ${ }^{199} \mathrm{Pt}$ decays into ${ }^{199} \mathrm{Au}$ with a half-life of 30.8 minutes. ${ }^{53}$ Solvent extraction with ethyl acetate or cation exchange can be used to separate $\mathrm{Au}$ (III) ions from their Pt(IV) 
counterparts in the dissolved target. After irradiation, the Pt target is dissolved in a glove box using $500 \mu \mathrm{L}$ of boiling aqua regia in a glass liquid scintillation vial. The resulting solid is redissolved in $500 \mu \mathrm{L}$ of $1 \mathrm{M} \mathrm{HCl}$ and again evaporated to dryness. The solid is prepared for extraction by addition of $500 \mu \mathrm{L}$ of $3 \mathrm{M} \mathrm{HCl}$ and transfer to a plastic V-bottom vial. Extraction occurs through addition of an aliquot of ethyl acetate (300 $\mu \mathrm{L})$, vortexing, and pipetting of the ethyl acetate layer. This process is repeated until adequate yield is achieved. After extraction, the ethyl acetate is evaporated and the remaining impurities are digested with $300 \mu \mathrm{L}$ of $35 \% \mathrm{H}_{2} \mathrm{O}_{2}$. The gold is then redispersed in $0.05 \mathrm{HCl}$ for use. The separation process has high yield (>90\%) and radiochemical purity (>99\%). ${ }^{56,57}$

\subsubsection{Chemical Reactivity of Gold}

Chemically, gold exhibits three easily accessible oxidation states. Most commonly, gold exists as solid, neutral gold. $\mathrm{Au}^{0}$ has historically been used in the production of coins, hence its designation as one of the unreactive coinage metals. Under harsh conditions, such as immersion in hot aqua regia, gold will oxidize to the $\mathrm{Au}^{+3}$ state in the form $\mathrm{AuCl}_{4}{ }^{-}$. Reduction of $\mathrm{AuCl}_{4}{ }^{-}$to form nanoparticles is favorable,

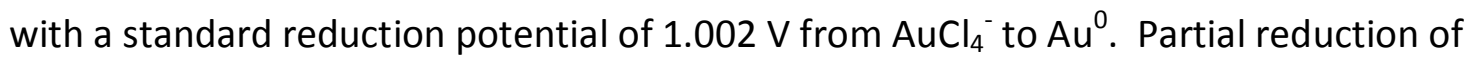
$\mathrm{AuCl}_{4}{ }^{-}$to $\mathrm{AuCl}_{2}^{-}(0.93 \mathrm{~V})$ produces $\mathrm{Au}^{+1}$ which can then be further reduced to $\mathrm{Au}^{0}(1.154$ V). ${ }^{58}$

While all three gold oxidation states are currently being investigated for potential therapeutic application, ionic gold shows instability in biologically relevant 
media. Reduction to colloidal gold is seen in many $\mathrm{Au}^{+3}$ and $\mathrm{Au}^{+1}$ compounds due to the body's natural redox potential. ${ }^{59,60}$ Stabilizing $\mathrm{Au}^{+3}$ in vivo presents an even more daunting challenge in light of proteins evolved to chelate trivalent metal ions such as $\mathrm{Fe}^{3+}$. For this reason, many groups are using neutral gold in nanoparticulate form for therapy. $\mathrm{Au}^{0}$ remains inert in vivo and, given the proper surfactant, can be highly water soluble. Gold nanoparticles are among the most studied and consequently best understood nanomaterials.

\subsubsection{Production, Nuclear, and Chemical Properties of ${ }^{64} \mathrm{Cu}$}

While ${ }^{199}$ Au provides SPECT capability, many applications call for the higher spatial resolution inherent from positron emission tomography (PET) imaging. This imaging requires a positron emitter such as ${ }^{64} \mathrm{Cu}$. Copper-64 decays by $\beta^{-}(38.4 \%), \beta^{+}$ (17.8\%), and electron capture (43.8\%) decay. Copper-64 can be produced from the ${ }^{64} \mathrm{Ni}(\mathrm{p}, \mathrm{n}){ }^{64} \mathrm{Cu}$ reaction on an enriched ${ }^{64} \mathrm{Ni}$ target at modest cyclotron energies (12 MeV). The reaction and separation via ion exchange chromatography produce ${ }^{64} \mathrm{Cu}$ with high radionuclidic purity (>99\%). ${ }^{61}$ The decay scheme of ${ }^{64} \mathrm{Cu}$ is shown in Figure 2.1.2.

$$
\begin{aligned}
& \beta^{-}, 38.4 \%, \quad \beta^{+}, 17.8 \% \text {, } \\
& { }^{64} \mathrm{Zn} \stackrel{573 \mathrm{keV}}{\longleftarrow}{ }^{64} \mathrm{Cu} \underset{\text { E.C., } 43.8 \%}{\stackrel{655 \mathrm{keV}}{\rightleftarrows}}{ }^{64} \mathrm{Ni}
\end{aligned}
$$

Figure 2.1.1: Simplified decay scheme of ${ }^{64} \mathrm{Cu}$. 
Ionic copper is more stable than ionic gold, and can be chelated with a number of different ligands. When chelated, copper exists predominantly in the +2 oxidation state. Nitrogen based, macrocyclic chelating agents such as $1,4,8,11-$ tetraazacyclotetradecane-1,4,8,11-tetraacetic acid (TETA) effectively bind $\mathrm{Cu}^{2+}$ in vitro, but show instability in vivo. The crossbridged analog to TETA, CB-TE2A, adds copper slowly but exhibits high stability in vivo. ${ }^{62}$ Substituting phosphonic acid arms for the acetic acid arms of the TE2A system increases the kinetic speed of $\mathrm{Cu}^{2+}$ incorporation while retaining high in vivo stability. ${ }^{62}$ Other radionuclides of copper also may prove useful for radiotherapy and imaging, with ${ }^{67} \mathrm{Cu}$ functioning as a $\beta^{-}$therapeutic and ${ }^{60} \mathrm{Cu}$ having potential as a PET radionuclide.

\subsubsection{Specific Aims}

The first portion of this work assessed the ability of non-specifically targeted nanoparticles to serve as brachytherapy agents. In these experiments, the gold nanoparticle surface is passivated by the biocompatible surfactant gum arabic. Nanoparticles are injected intratumorally (I.T.) and monitored for retention in tumor tissue over a 1 month period. Rather than painfully inserting hundreds of macroscopic seeds into the prostate, a single injection of nanoparticles delivers a prodigious number of microscopic seeds directly to the tumor.

The second portion of this work evaluates a specifically targeted $\beta^{-}$therapeutic consisting of ${ }^{198 / 199}$ Au nanoparticles coated with starch and directed in vivo by a 
truncated bombesin targeting agent. Targeting efficacy is monitored by multiple injection pathways in comparison to untargeted nanoparticles and bombesin-targeted bifunctional chelate constructs. Bombesin targets the gastrin-releasing peptide (GRP) receptors in cells. These GRP receptors are over expressed in prostate cancer cells, with as many as 44,000 receptors per PC-3 prostate cancer cell. ${ }^{63}$ While the full length bombesin peptide consists of 14 amino acids, truncated peptides based on amino acids 7-14 show improved targeting and pharmacokinetics as a result of amino acid substitution or modified chelation/spacer groups. ${ }^{64}$ The full length bombesin peptide (Pyr-Gln-Arg-Leu-Gly-Asn-Gln-Trp-Ala-Val-Gly-His-Leu-Met- $\mathrm{NH}_{2}$ ) is shown in Figure 2.1.1.

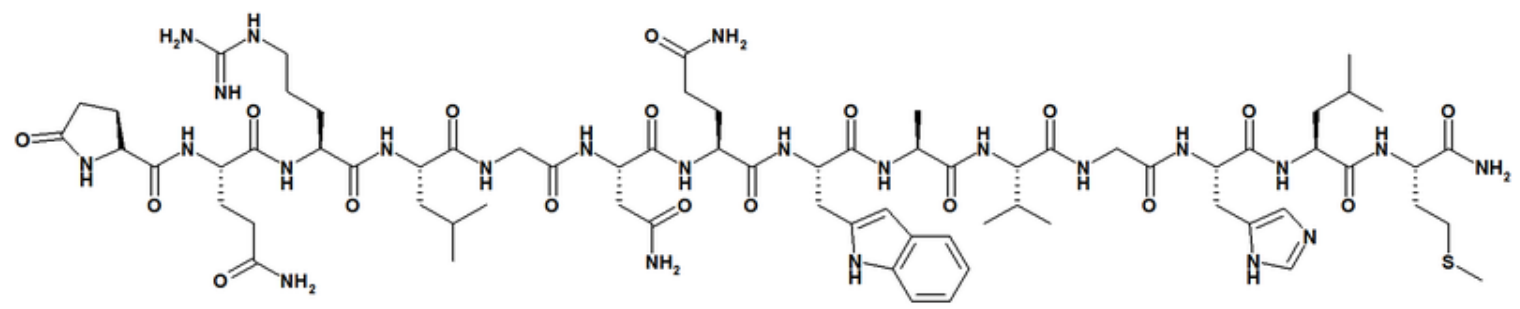

Figure 2.1.2: Structure of the 14 amino acid bombesin peptide.

The third portion of this work focuses on development of a ${ }^{64} \mathrm{Cu}$ containing gold nanoparticle. Radiometal chelation with non-macrocyclic compounds such as diethylene triamine pentacetic acid (DTPA) can prove unstable in vivo. ${ }^{65}$ Binding multiple DTPA chelates to a single nanoparticle could provide a sandwich style structure which would sufficiently stabilize the ${ }^{64} \mathrm{Cu}$. This development would generate a PET imaging agent with the passive targeting advantages of a nanoparticle. 


\subsection{Gum Arabic Gold Nanoparticle Experimental}

\subsubsection{Materials}

All chemicals were obtained from Sigma-Aldrich and were at least American Chemical Society (ACS) grade. Materials were used as received without further purification or modification. All animal experiments were conducted according to the procedures set forth by the Institutional Animal Care and Use Committee of the University of Missouri-Columbia.

\subsubsection{Gum Arabic Gold Nanoparticle Synthesis}

Gum arabic (2.94 mg) was dissolved in $6 \mathrm{~mL}$ of $18 \mathrm{M} \Omega$ water. $\mathrm{NaAuCl}_{4}(100 \mu \mathrm{L}$, $0.1 \mathrm{M}$ ) was added to the gum arabic solution in a $20 \mathrm{~mL}$ glass scintillation vial. Trishydroxylmethyl phosphine alanine (THPAL, $3.9 \mathrm{mg}$ ) was dissolved in $116 \mu \mathrm{L}$ of water. The dissolved gum arabic solution was heated under reflux, then $60 \mu \mathrm{L}$ of THPAL solution was added. A deep purple color forms immediately upon addition of the THPAL. The nanoparticle solution was heated for one minute, then cooled with stirring for 30 minutes. The solution was neutralized to physiological $\mathrm{pH}$ by addition $1 \mathrm{M} \mathrm{NaOH}$ and $\mathrm{HCl}$. Dulbecco's phosphate buffered saline (DPBS) was added to buffer the sample and restore the volume to $6 \mathrm{~mL}$. The nanoparticle solution was diluted by taking $100 \mu \mathrm{L}$ of solution and mixing with $900 \mu \mathrm{L}$ of water for Ultraviolet-visible (UV-Vis) measurements on an Ocean Optics USB 2000 spectrometer. 


\subsubsection{Biodistribution and Animal Studies}

Biodistribution studies were performed by Lisa Watkinson and Terry Carmack under the supervision of Prof. John Lever and Dr. Jeff Smith at the Harry S Truman Memorial Veterans Affairs Hospital. The non-radioactive gum arabic coated nanoparticles $(30 \mu \mathrm{L})$ were injected I.T. into randomly selected female imprinting control region severe combined immunodeficient (ICRSCID) mice $(n=7)$ with average body mass of $23.01 \pm 1.76 \mathrm{~g}$. Human PC-3 prostate cancer xenografts were grown in the mice prior to injection with average tumor volume of $0.052 \pm 0.029 \mathrm{~g}$. PC-3 cells were obtained from American Type Culture Collection and were maintained by the University of Missouri Cell and Immunobiology Core Facility. Control mice $(n=7)$ were injected with $30 \mu \mathrm{L}$ of DPBS solution I.T. Over the next month, tumors were measured using external calipers to estimate tumor volume. Additionally, animal weight was monitored as an indicator of overall health. If an animal experienced drastic weight loss, it was euthanized and its organs harvested. After 30 days, the surviving mice were sacrificed and their organs harvested (bladder, kidney, small intestine, large intestine, liver, lung, pancreas, spleen, muscle, and tumor) for analysis by neutron activation analysis (NAA). Each organ was freeze dried and loaded into a $2 / 5$ dram $(1.48 \mathrm{~mL})$ polyethylene vial before co-irradiation with gold standards. Samples were allowed to decay for three hours before counting. 


\subsection{Gum Arabic Gold Nanoparticle Results and Discussion}

Gum arabic coated gold nanoparticles exhibited a plasmon resonance absorption maximum at $539 \mathrm{~nm}$. This absorption corresponds with the absorption of gum arabic nanoparticles previously measured by scanning transmission electron microscopy (TEM) of $\sim 15 \mathrm{~nm}$ in diameter. Previous dynamic light scattering (DLS) measurements indicate that these $15 \mathrm{~nm}$ nanoparticles have a hydrodynamic diameter of $85 \mathrm{~nm}$ and a zeta potential of $-24.5 \mathrm{mV} .^{57}$

During the experiments, two mice from the nanoparticle treatment group and two mice from the DPBS control group showed significant weight loss and were euthanized before the end of the thirty day trial period. Within the surviving mouse population, nanoparticles injected into the tumor showed long residence times, with $80.0 \pm 18.7 \%$ of the injected dose (ID) retained over the 1 month time period. Nanoparticles which did not remain in the tumor were excreted primarily via the reticuloendothelial system (RES). Next to the tumor, the highest organ uptake following IT injection occurred in the liver, with $1.4 \pm 0.4 \%$ ID present. Uptake in the spleen $(0.11$ $\pm 0.01 \% \mathrm{ID})$ is also indicative of the RES clearance pathway. The total amount of gold detected in the collected organs accounted for $83.9 \%$ of the initial ID. Undetected gold nanoparticles were presumably present in small amounts in the remainder of the animal or excreted over the one month study period. The average minimum detectable amount of gold in tissue via the NAA measurements was approximately $10 \mathrm{ng}$. The biodistribution of the particles is shown in Figure 2.3.1. 


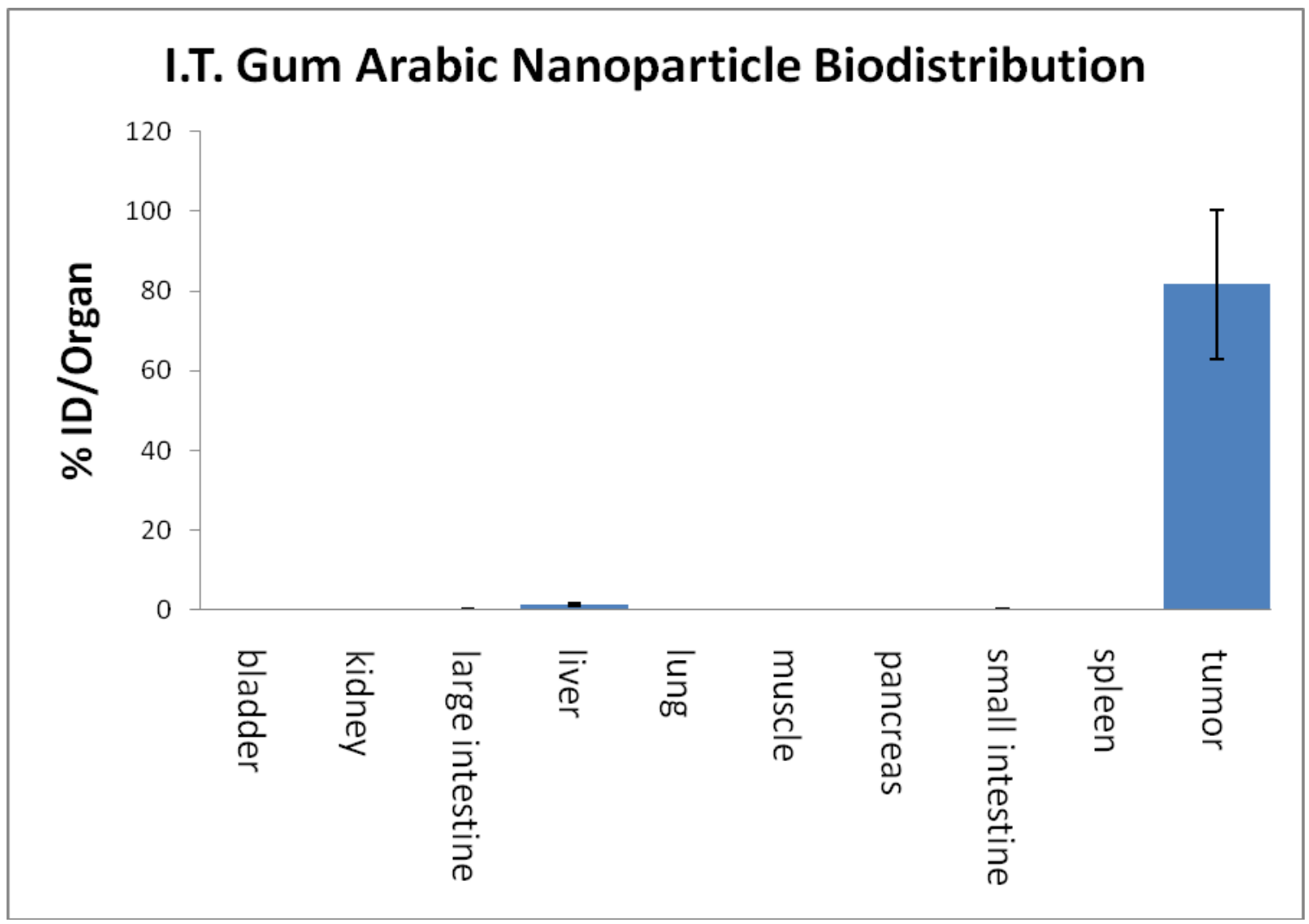

Figure 2.3.1: Gum arabic coated gold nanoparticles present in SCID mice one month following I.T. injection in PC-3 xenografts measured by NAA.

Non-radioactive gum arabic coated gold nanoparticles are retained in the tumor to a much higher degree than radioactive nanoparticles. Previous therapy studies done by Chanda, et al. utilizing ${ }^{198} \mathrm{Au}$ gum arabic nanoparticles indicate that only $19.9 \pm 4.2 \%$ of the ID was retained in the tumor after one month. ${ }^{66}$ In the radioactive study, most of the tumor tissue was necrotic at this point, which was not observed with the nonradioactive nanoparticles. The difference may possibly be explained through differences in clearance between the necrotic tissue found in mice treated with radioactive 
nanoparticles and the viable tumor tissue found in mice treated with non-radioactive nanoparticles.

Tumor sizes between groups receiving DPBS and non-radioactive gold nanoparticles showed no statistically significant difference in tumor volumes or mass ( $p$ value $=0.1258)$. In the group receiving gold nanoparticles, tumor volume averaged 0.65 $\pm 0.21 \mathrm{~cm}^{3}$ while in the group receiving PBS, tumor volume averaged $0.49 \pm 0.43 \mathrm{~cm}^{3}$. After sacrifice, tumors from the treatment group had an average mass of $0.888 \pm 0.193$ $\mathrm{g}$ while tumors from the PBS group had an average mass of $0.704 \pm 0.225 \mathrm{~g}$. These results are shown in Figures 2.3.2 $\mathrm{A}$ and $\mathrm{B}$.

Gum arabic coated ${ }^{198} \mathrm{Au}$ nanoparticles have shown tremendous potential to shrink tumors in unresectable prostate cancer. ${ }^{67}$ However, it had not been rigorously established that this tumorcidal effect was due to the radioactivity present rather than to some innate cytotoxicity of the gold nanoparticles. While some gold nanoparticles show cytotoxicity, this most often occurs in cationic particles of $<2 \mathrm{~nm}$ diameter. ${ }^{68}$ In this case, cytotoxicity results from the small positively charged nanoparticle disrupting the negatively charged cellular membrane. ${ }^{69}$ Membrane disruption inhibits the cell's ability to maintain homeostasis and regulate its environment, ultimately leading to cell death. 


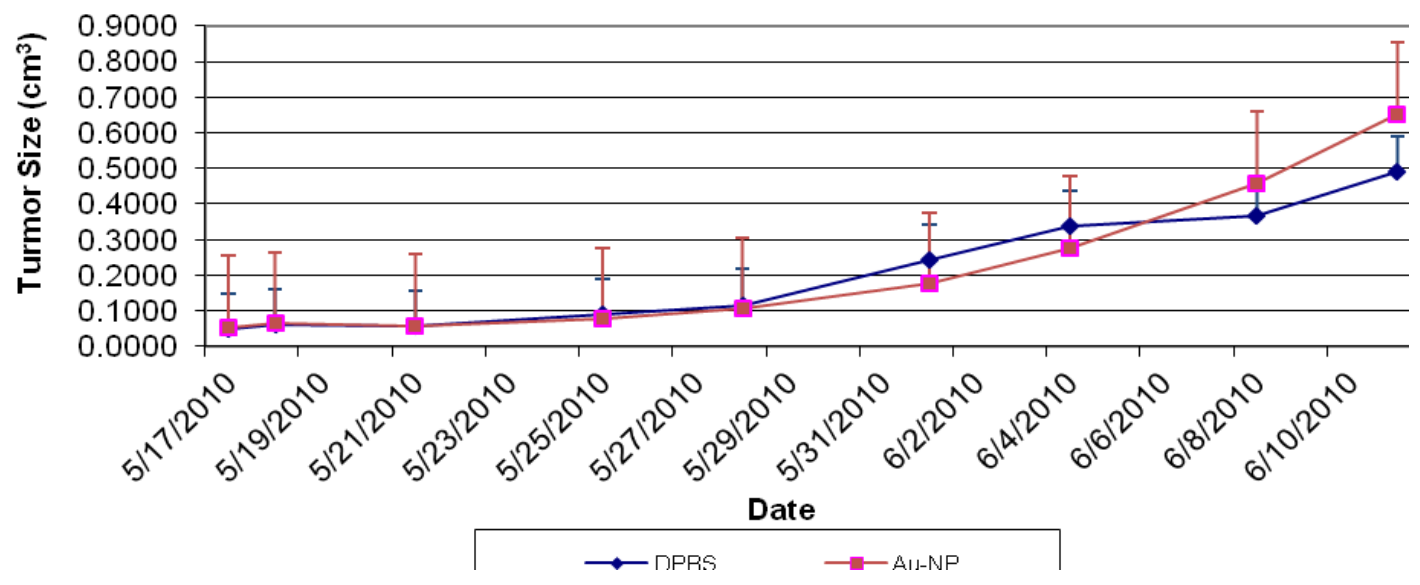

B

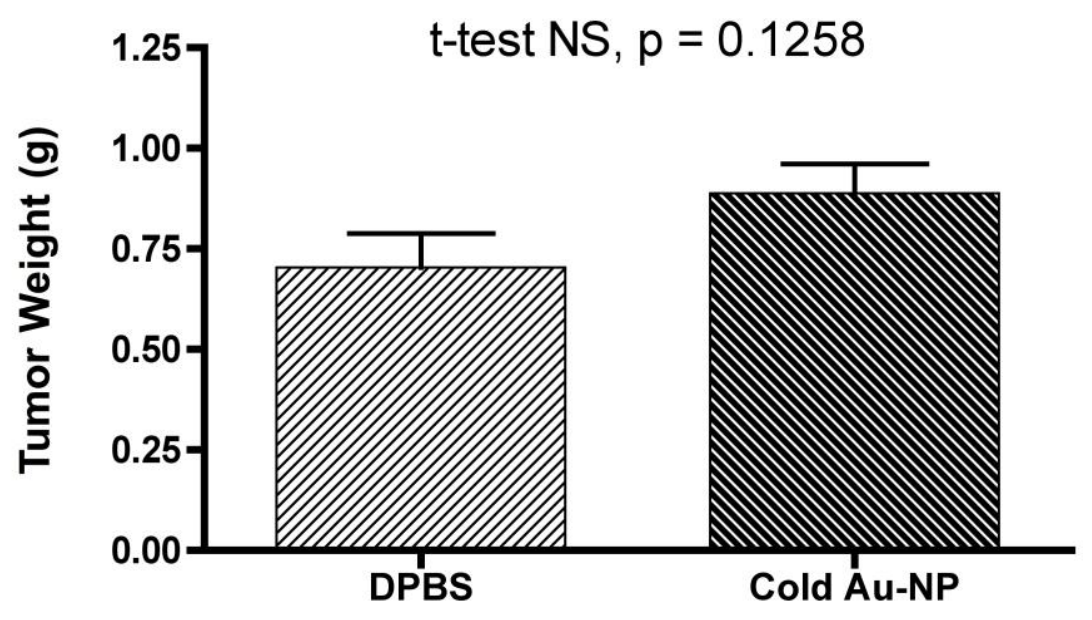

Figure 2.3.2 A and B: Tumor volume (A) and Tumor mass (B) after treatment with gum arabic gold nanoparticles. No significant difference was observed between mice treated with DPBS and mice treated with non-radioactive gum arabic coated gold nanoparticles.

The gum arabic nanoparticles used in the experiment show no statistically significant cytotoxic effect on the tumor. Additionally, the nanoparticle group showed 
no additional weight loss or other symptom relative to the DPBS group. Two mice from each group were sacrificed early as a result of deteriorating health and tumor associated weight loss. Therefore, radioactivity from ${ }^{198} \mathrm{Au}$ is responsible for the tumor shrinking properties seen in I.T. injections, rather than any chemical properties of the nanoparticles.

\subsection{Starch-Bombesin Gold Nanoparticle Experimental}

\subsubsection{Materials and Radiogold Production}

All chemicals were obtained from Sigma-Aldrich and were at least ACS grade unless otherwise noted. Materials were used as received without further purification or modification. Water (18 M $\Omega$ ) was produced from an in-house system. Bombesin 7-14 (hereafter referred to as simply bombesin) was purchased from Anaspec. Radiogold was produced by irradiating a $0.75 \mathrm{mg}$ gold foil at the University of Missouri Research Reactor (MURR) for 6 hours in a thermal neutron flux of $8^{*} 10^{13} \mathrm{n} / \mathrm{cm}^{2} / \mathrm{s}$. After irradiation, the quartz vial containing the gold target was washed of contaminant radioactivity and opened in a glove box where it was dissolved in $800 \mu \mathrm{L}$ of aqua regia. The aqua regia was evaporated and the gold dissolved in $400 \mu \mathrm{L}$ of $1.0 \mathrm{M} \mathrm{HCl}$. This $\mathrm{HCl}$ was evaporated and the gold reconstituted in $800 \mu \mathrm{L}$ of $0.05 \mathrm{M} \mathrm{HCl}$.

\subsection{2 ${ }^{64} \mathrm{Cu}$-Bombesin Conjugate Synthesis}

$\left[{ }^{64} \mathrm{Cu}-1,4,7-T r i a z a c y c l o n o n a n e-1,4-d i a c e t i c\right.$ acid (NO2A)-bombesin $]$ conjugates 
were synthesized by Prof. Jeff Smith's group at the Harry S Truman Veterans Affairs Hospital. ${ }^{70}$ The synthesis was conducted by adding ${ }^{64} \mathrm{CuCl}_{2}\left(260 \mathrm{MBq}, 2.83 \times 10^{-11} \mathrm{~mol}\right.$, MDS Nordion) to a plastic tube containing bombesin-NO2A conjugate ( $20 \mu \mathrm{g})$ and 250 $\mu \mathrm{L}$ of $0.4 \mathrm{M}$ ammonium acetate. The $\mathrm{pH}$ of the reaction mixture was adjusted to $\sim 7$ by the addition of $2 \mathrm{mM} \mathrm{NaOH}$ then incubated $\left(80^{\circ} \mathrm{C}, 1 \mathrm{~h}\right)$. Fifty microliters of $10 \mathrm{mM}$ DTPA solution was added to scavenge unbound radioactivity. The radiolabeled conjugates were then purified by reverse phase high performance liquid chromatography and collected into $100 \mu \mathrm{L}$ of $1 \mathrm{mg} / \mathrm{ml}$ bovine serum albumin (BSA) stabilizing agent with 25 $\mu \mathrm{g}$ ascorbic acid. Figure 2.4 .1 shows the synthetic conjugate. ${ }^{70}$<smiles>CSCCC(NC(=O)C(CC(C)C)NC(=O)C(Cc1cnc[nH]1)NC(=O)CNC(=O)C(NC(=O)C(C)NC(=O)C(Cc1c[nH]c2ccccc12)NC(=O)C(CCC(N)=O)NNONC(=O)CN1CCN2CC(=O)OC12N1CCN2CC(=O)OC21C)C(C)C)C(N)=O</smiles>

Figure 2.4.1: Molecular structure of ${ }^{64} \mathrm{Cu}-\mathrm{NO} 2 \mathrm{~A}-\mathrm{Bombesin}$ conjugate

\subsubsection{Starch Gold Nanoparticle Synthesis}

Starch (12.5 mg) was dissolved in $6 \mathrm{~mL}$ of $18 \mathrm{M} \Omega$ water. $\mathrm{NaAuCl}_{4}$ carrier $(95 \mu \mathrm{L}$ of $0.1 \mathrm{M}$ solution in water) and $\mathrm{H}^{198} \mathrm{AuCl}_{4}(190 \mathrm{MBq}$ in $225 \mu \mathrm{L}$ of $0.05 \mathrm{M} \mathrm{HCl}$ ) were added to a $20 \mathrm{~mL}$ glass scintillation vial. The solution was neutralized by adding $10 \mu \mathrm{L}$ of $1 \mathrm{M}$ $\mathrm{NaOH}$, then heated under boiling conditions. THPAL solution $(30 \mu \mathrm{L}, 0.1 \mathrm{M})$ was added 
and the solution was heated for an additional minute. The solution was cooled with stirring for 30 minutes then analyzed by UV-Vis spectroscopy. Two $\mathrm{mL}$ of this solution was placed on a size exclusion column (300 mg Sephadex G-100 resin in $7 \mathrm{~mL}$ water prepared in a $9 \mathrm{~cm}$ Bio-Rad Poly-Prep polyethylene column) and eluted with $2 \mathrm{~mL}$ water. The $2 \mathrm{~mL}$ of eluent collected had an activity of $12 \mathrm{MBq}$. The solution was diluted with DPBS to achieve an activity concentration of $1.9 \mathrm{MBq} / \mathrm{mL}$. Gum arabic particles were synthesized as a comparator using the method described in 2.2.2 and similarly characterized.

\subsubsection{Bombesin Conjugation}

Thioctic acid-linked bombesin was synthesized by Dr. Raghuraman Kannan. ${ }^{71}$ The thioctic acid-bombesin (3.9 mg) was dissolved in $2 \mathrm{~mL}$ ethanol. Five hundred $\mu \mathrm{L}$ of the starch nanoparticles after size exclusion were added to $1 \mathrm{~mL}$ of the bombesin solution and allowed to stir overnight. The nanoparticles agglomerated into a pellet overnight. The supernatant was removed and the pellet was washed $3 x$ with $1 \mathrm{~mL}$ of ethanol to remove any excess bombesin. The bombesin peptide is soluble in ethanol while the nanoparticle-bombesin conjugates are not. Each washing consisted of vortexing, centrifuging, and decanting the remaining supernatant via pipette. The pellet was then washed in a similar manner $3 x$ with $18 \mathrm{M} \Omega$ water to remove any unconjugated nanoparticles. 
After washing, particles were analyzed by UV-Vis spectroscopy to confirm a change in gold surface environment following the reaction. The sample was redispersed in $500 \mu \mathrm{L}$ of dimethyl sulfoxide and $1.5 \mathrm{~mL}$ of DPBS for a total volume of $2 \mathrm{~mL}$ and an activity of 9.4 MBq. The $\mathrm{pH}$ of the solution was adjusted to between 7-8 with $1 \mathrm{M} \mathrm{NaOH}$ and $\mathrm{HCl}$. Nanoparticles were then diluted with DPBS to achieve the desired activity concentration of $1.9 \mathrm{MBq} / \mathrm{mL}$.

\subsubsection{Nanoparticle Characterization}

Nanoparticles were characterized via UV-Vis spectroscopy as in 2.2.3. Starch nanoparticles were analyzed by radio- thin layer chromatography (TLC) to quantify the amount of gold incorporated into the nanoparticle using a Bioscan B-AR-2000-2. Two $\mu \mathrm{L}$ of nanoparticle solution was placed at the bottom of a silica TLC plate and developed in methanol with 2 drops of concentrated $\mathrm{HCl}$. Due to their large size, the gold nanoparticles remain at the origin while ionic gold travels up the TLC plate with an $R_{f}$ of 0.66. Synthetic yield was determined from the relative peak areas. The peaks were integrated with the provided WinScan software. Nanoparticles were analyzed both before and after passage through the size exclusion column to confirm the removal of unbound gold.

\subsubsection{Biodistribution and Animal Studies}

Biodistribution studies were performed by Lisa Watkinson and Terry Carmack under the supervision of Prof. John Lever and Dr. Jeff Smith at the Harry S Truman 
Memorial Veterans Affairs Hospital. Four distinct radioactive agents were injected intravenously (I.V.) into SCID mice bearing human prostate tumor (PC-3) xenografts. PC3 cells were obtained from the American Type Culture Collection and cultured by the University of Missouri Cell and Immunobiology Core. Mice were prepared by subcutaneous implantation of $10,000,000$ PC-3 cells into the right flank. Solid tumors were allowed to develop for 3 weeks. For the first treatment group, $100 \mu \mathrm{L}$ of PBS was injected I.V. into four mice followed by intraperitoneal (I.P.) administration of $130 \mathrm{kBq}$ of ${ }^{198}$ Au gum arabic gold nanoparticles in $100 \mu \mathrm{L}$ PBS to determine the biodistribution of passively targeted nanoparticles. An additional four mice received $100 \mu \mathrm{L}$ of bombesin peptide 15 minutes before I.P. administration of $130 \mathrm{kBq}$ of ${ }^{198} \mathrm{Au}$ gum arabic nanoparticles in $100 \mu \mathrm{L}$ of PBS.

Starch capped nanoparticles consisted of four different injection groups of five mice each. The first group received $100 \mu \mathrm{L}$ of PBS I.V. 15 minutes prior to I.P administration of $130 \mathrm{kBq}$ of starch capped ${ }^{198} \mathrm{Au}$ nanoparticles in $100 \mu \mathrm{L}$ of PBS. Group 2 received $100 \mu \mathrm{L}$ of bombesin peptide I.V. 15 minutes prior to an identical nanoparticle administration as group 1. The third group received PBS I.V. 15 minutes before I.P. administration of $130 \mathrm{kBq}$ of ${ }^{198} \mathrm{Au}$ starch-bombesin coated nanoparticles in $100 \mu \mathrm{L}$ of PBS. The final group received I.V. administration of $100 \mu \mathrm{L}$ bombesin peptide 15 minutes before I.P. administration of $130 \mathrm{kBq}$ of ${ }^{198} \mathrm{Au}$ starch-bombesin coated nanoparticles in $100 \mu \mathrm{L}$ of PBS. 
Additionally, four groups of three mice each received $190 \mathrm{kBq}(20 \mathrm{ng})$ of ${ }^{64} \mathrm{Cu}$ bombesin conjugate in $100 \mu \mathrm{L}$ of PBS. Group 1 was injected I.V. with $100 \mu \mathrm{L}$ of PBS before conjugate administration I.V. The second group received $100 \mu \mathrm{L}$ of bombesin peptide I.V. before I.V. conjugate administration. Group 3 was injected with $100 \mu \mathrm{L}$ PBS before I.P. administration of conjugate. Finally, group 4 received $100 \mu \mathrm{L}$ of bombesin peptide before I.P conjugate injection. All mice were sacrificed 2 hours after injection of activity and dissected. Organs and tissue (blood, heart, lung, liver, spleen, large intestine, pancreas, and tumor) were collected for analysis by $\gamma$ spectroscopy. A summary of the injections is presented in Table 2.4.1

Table 2.4.1: Summary of bombesin injections

\begin{tabular}{|l|c|c|c|c|}
\hline Treatment & \# of mice & Pretreatment & Nuclide & Activity (kBq) \\
\hline Gum arabic NP, I.P. & 4 & PBS, I.V. & ${ }^{198} \mathrm{Au}$ & 130 \\
\hline Gum arabic NP, I.P. & 4 & Bombesin, I.V. & ${ }^{198} \mathrm{Au}$ & 130 \\
\hline Starch NP, I.P. & 5 & PBS, I.V. & ${ }^{198} \mathrm{Au}$ & 130 \\
\hline Starch NP, I.P. & 5 & Bombesin, I.V. & ${ }^{198} \mathrm{Au}$ & 130 \\
\hline Starch-BBN NP, I.P. & 5 & PBS, I.V. & ${ }^{198} \mathrm{Au}$ & 130 \\
\hline Starch-BBN NP, I.P. & 5 & Bombesin, I.V. & ${ }^{198} \mathrm{Au}$ & 130 \\
\hline${ }^{64} \mathrm{Cu}$-bombesin & 3 & PBS, I.V. & ${ }^{64} \mathrm{Cu}$ & 190 \\
\hline${ }^{64} \mathrm{Cu}$-bombesin & 3 & Bombesin, I.V. & ${ }^{64} \mathrm{Cu}$ & 190 \\
\hline${ }^{64} \mathrm{Cu}$-bombesin & 3 & PBS, I.V. & ${ }^{64} \mathrm{Cu}$ & 190 \\
\hline${ }^{64} \mathrm{Cu}$-bombesin & 3 & Bombesin, I.V. & ${ }^{64} \mathrm{Cu}$ & 190 \\
\hline
\end{tabular}




\subsection{Starch-Bombesin Gold Nanoparticle Results and Discussion}

\subsubsection{Starch Gold Nanoparticle Yield and Characterization}

Starch coated gold nanoparticles showed a UV-Vis absorbance maximum at 544 $\mathrm{nm}$. After filtration, the solution showed an absorbance maximum at $541 \mathrm{~nm}$. During the synthesis, a small fraction of starch particles will polymerize, creating long chains of linked nanoparticles. These oligonanoparticles represent the longer wavelength absorbance. After filtration, the absorption maximum decreases in intensity due to lower number of particles present and also shifts towards the blue end of the spectrum signifying the smaller size of the remaining particles (Figure 2.5.2). Additionally, ionic gold present in the unfiltered sample remains on the sephadex column, as shown in Figure 2.5.1 A and B.
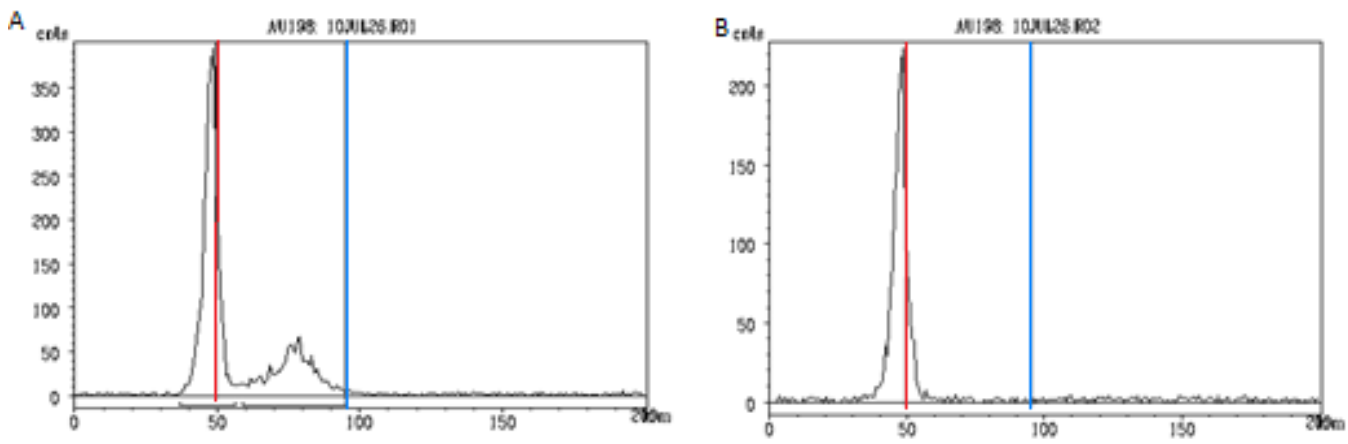

Figures 2.5.1 A and B: Radio-TLC of starch coated gold nanoparticles before (A) and after (B) filtration. The origin on each plate is at $50 \mathrm{~mm}$ and marked by a red line while the solvent front is shown by the line at $95 \mathrm{~mm}$ and marked by a blue line. 
After conjugation to bombesin, the nanoparticles were washed successively with ethanol and water to remove both unconjugated bombesin and free particles. The activity in the supernatant in each wash is shown in Table 2.5.1.

Table 2.5.1: Activity in each wash of bombesin coated gold nanoparticles

\begin{tabular}{|l|l|}
\hline Wash & Activity $(\mathrm{kBq})$ \\
\hline Initial supernatant & 318 \\
\hline $1^{\text {st }}$ ethanol & 44 \\
\hline $2^{\text {nd }}$ ethanol & 41 \\
\hline $3^{\text {rd }}$ ethanol & 74 \\
\hline $1^{\text {st }}$ water & 1890 \\
\hline $2^{\text {nd }}$ water & 1300 \\
\hline $3^{\text {rd }}$ water & 370 \\
\hline
\end{tabular}

The ethanol washes were to remove unconjugated peptide and showed small activity breakthrough, indicating that very few gold nanoparticles were removed in the process. Water washes, meant to remove nanoparticles without bombesin, showed successively decreasing activity in each wash indicating the progressive removal of unconjugated nanoparticles. Removal of both conjugated and unconjugated nanoparticles in the wash would remove a constant fraction of activity rather than a decreasing amount. The final nanoparticle solution showed a UV-Vis absorbance of 0.291 at $561 \mathrm{~nm}$, reflecting the addition of the large bombesin conjugate. UV-Vis Spectra are shown in Figure 2.5.2. 


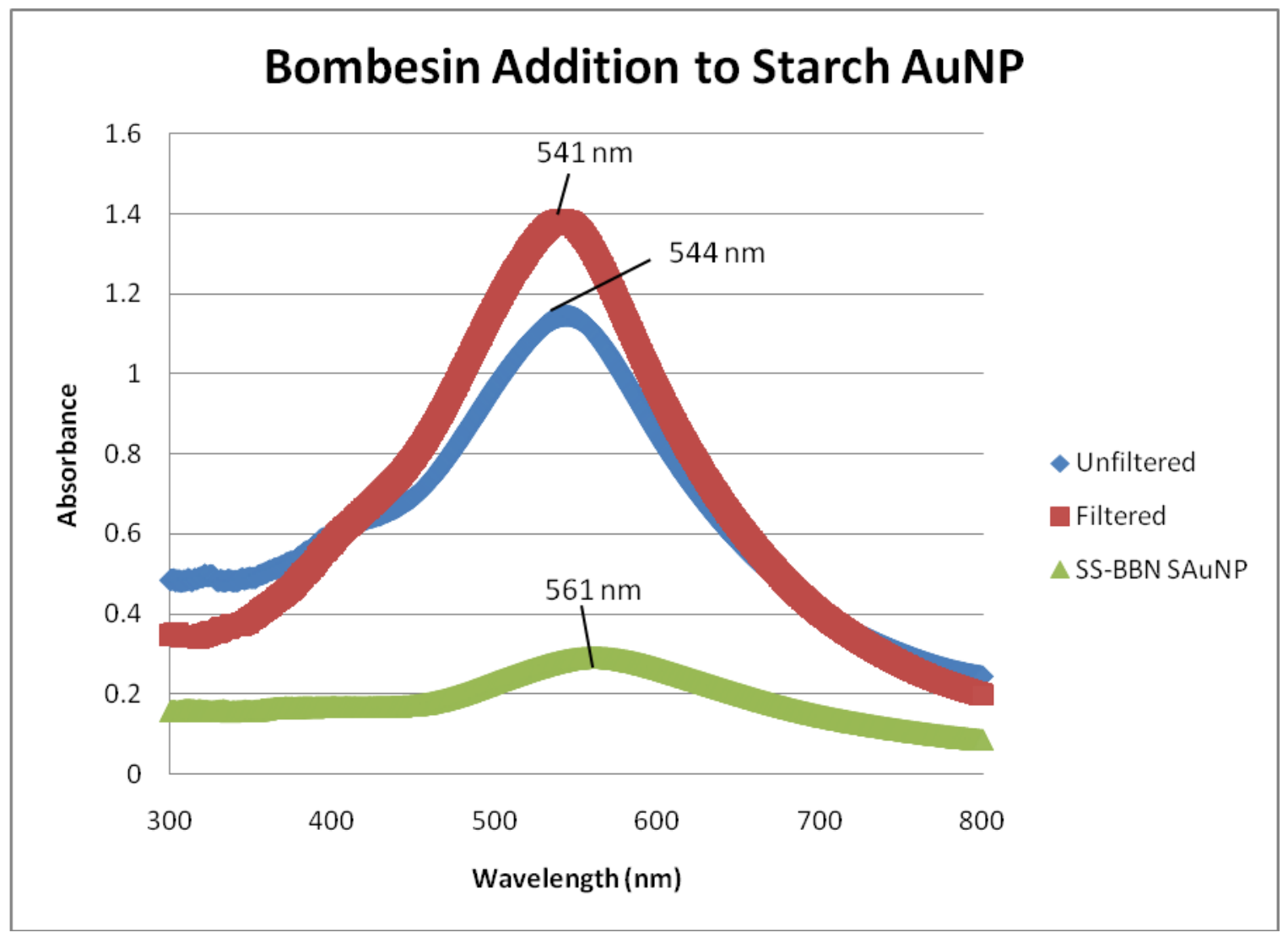

Figure 2.5.2: Shift of the plasmon resonance UV-Vis peak of the nanoparticle after starch addition, filtration, and bombesin addition to the surface.

\subsubsection{Gum Arabic Gold Nanoparticle Yield and Characterization}

The gum arabic gold nanoparticles gave a peak absorbance at $524 \mathrm{~nm}$. Nanoparticles were made with $87 \%$ yield, as measured by radio-TLC (Figure 2.5.3). 


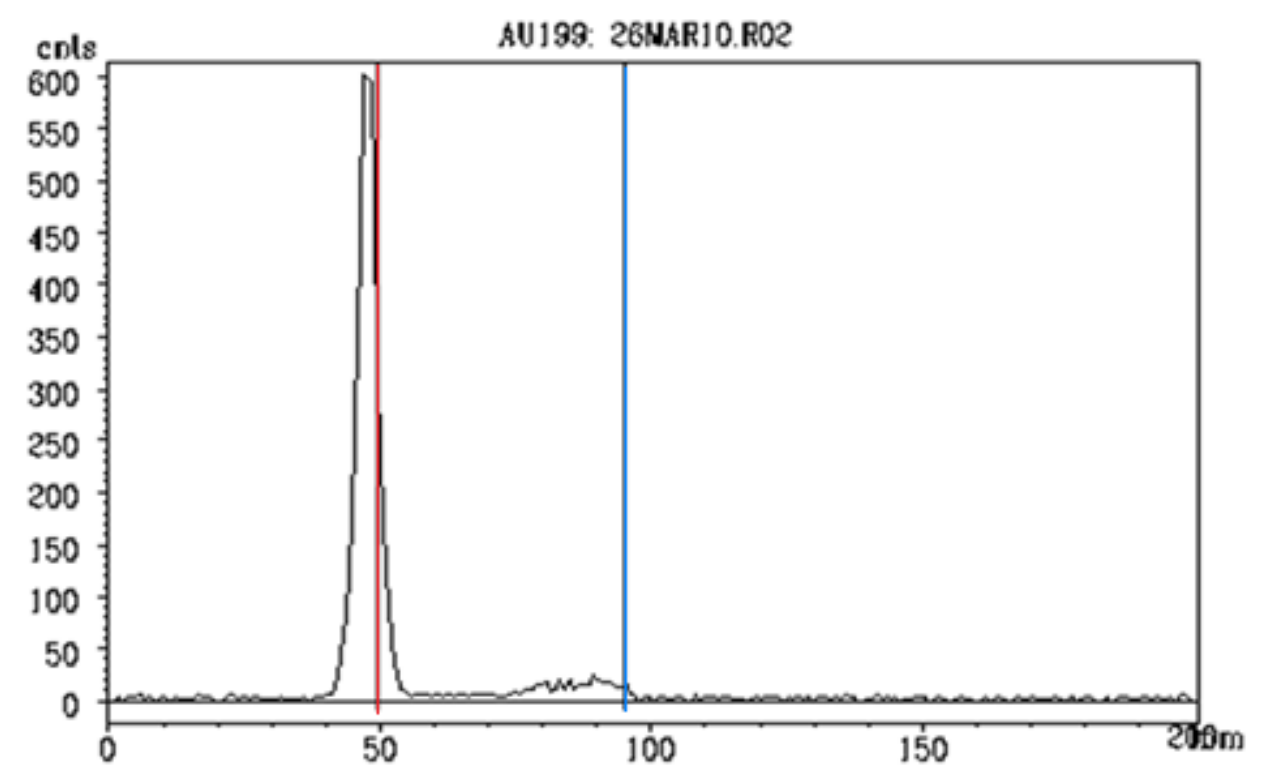

Figure 2.5.3: Radio-TLC of ${ }^{198} \mathrm{Au}$ gum arabic gold nanoparticles. The origin of the TLC place is at $50 \mathrm{~mm}$ (red line) while the solvent front is at $95 \mathrm{~mm}$ (blue line).

\subsubsection{Biodistribution and Animal Studies}

Injections of ${ }^{64} \mathrm{Cu}$-bombesin showed expected uptake patterns comparable to those previously seen. ${ }^{70}$ Bombesin targets a GRP receptor subtype which is highly expressed in endogenous murine tissue, particularly on the pancreas and large intestine. ${ }^{72}$ As such, these organs are in fact better exemplars of bombesin-specific targeting than the tumor xenograft itself. Human pancreas and large intestine display a different GRP receptor subtype which is not targeted by bombesin. Most of the uptake in the ${ }^{64} \mathrm{Cu}$ conjugate occurs in the pancreas and large intestine, indicating targeting of GRP receptors. Further, after blocking with free bombesin peptide, intestinal and pancreatic uptake show dramatic, statistically significant decreases (at the 95\% 
confidence level) in both the I.V. and I.P. injection pathways indicating receptor specific targeting. Blocking GRP receptors did not induce a statistically significant change in uptake in the tumor xenograft. Deviations between animals in each injection group are small. Biodistribution data for each organ and injection pathway are shown in Figure 2.5.4.

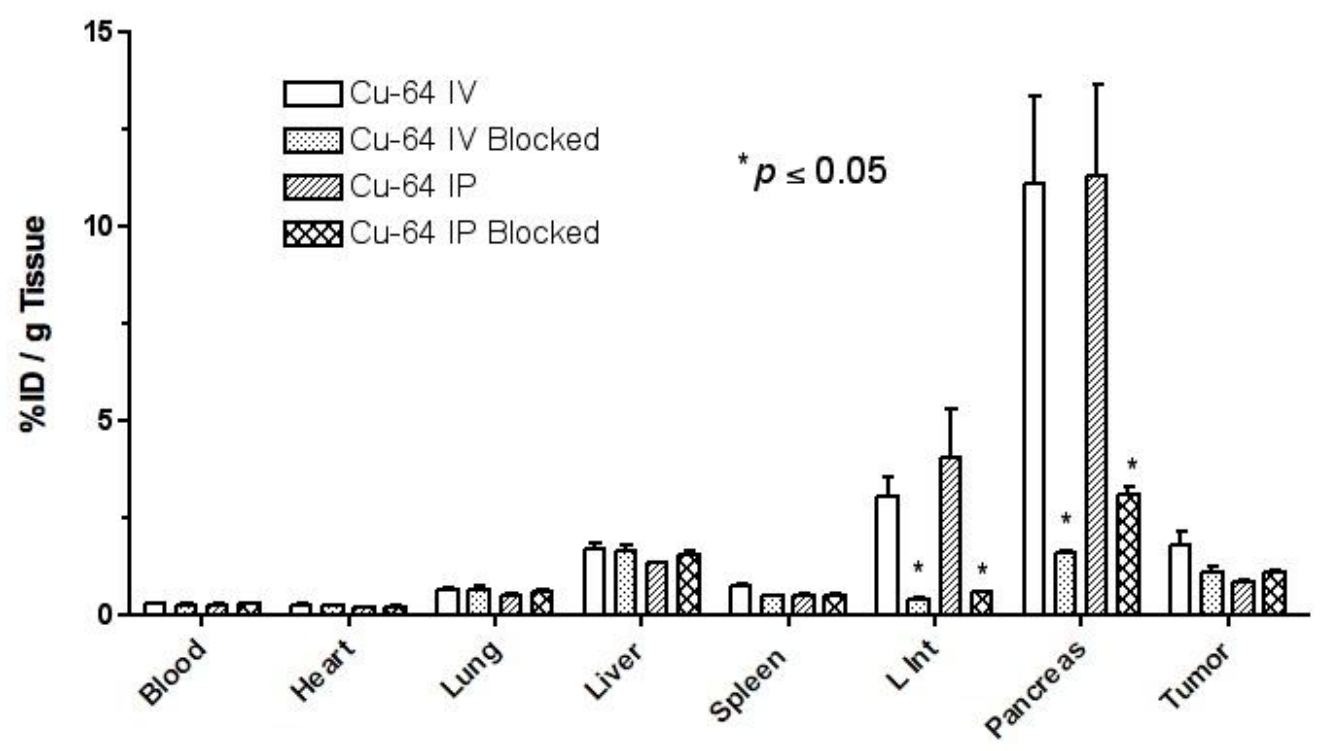

Figure 2.5.4: Blocking studies of ${ }^{64} \mathrm{Cu}$-bombesin conjugates using both I.V. and I.P. injection pathways. Injecting free bombesin peptide before particle injection produced statistically significant differences in pancreatic and intestinal uptake.

Passively targeted ${ }^{198} \mathrm{Au}$ gum arabic coated gold nanoparticles exhibit biodistribution patterns similar to those observed previously. ${ }^{57}$ Because the gum arabic is tightly bound to the gold nanoparticle, it is not possible to coordinate bombesin using 
the same chemistry as in the starch nanoparticle case. Without specific targeting, tumor uptake is low after only 2 hours of circulation time. Nanoparticles are cleared primarily through the RES, as evidenced by the large uptake in the liver and spleen.

Biodistribution data for ${ }^{198} \mathrm{Au}$ gum arabic coated particles is shown in Figure 2.5.5 both with and without blocking by unconjugated bombesin peptide.

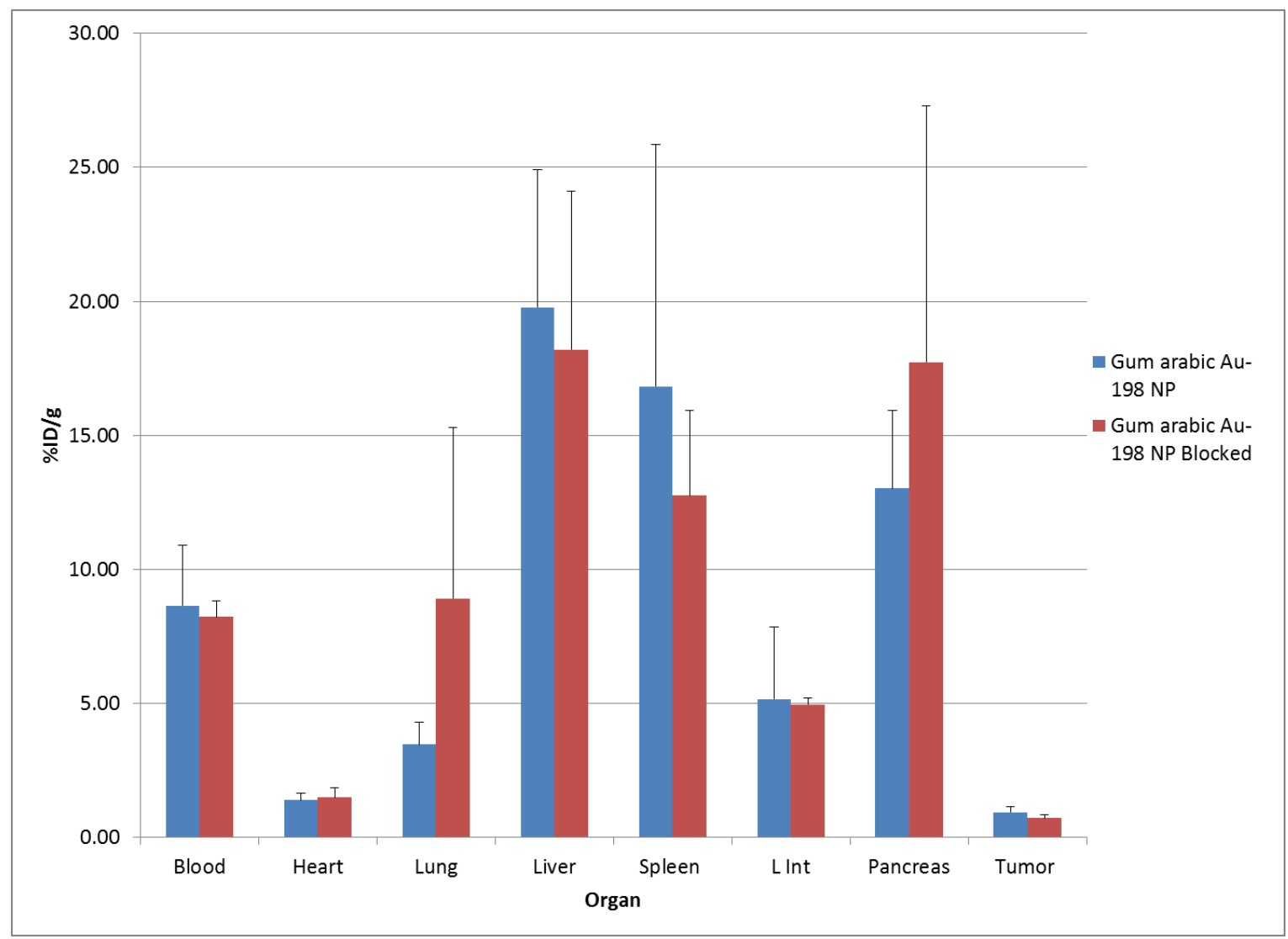

Figure 2.5.5: Biodistribution of gum arabic coated gold nanoparticles. These nanoparticles show uptake and clearance typical of passively targeted colloidal systems.

Passively targeted starch coated gold nanoparticles mirror uptake observed in the gum arabic system. Primary clearance pathway is through the RES, as evidenced by 
similar uptake in liver and spleen. No statistically significant differences in uptake were observed by blocking the GRP receptor with excess bombesin.

Starch binds less tightly to the surface of gold than gum arabic, and can be displaced by a lipoic acid-bombesin conjugate for specific targeting. Unfortunately, this weaker starch binding may also lead to dissociation of the starch from the gold in vivo and increased particle aggregation. Addition of bombesin to the starch nanoparticles should increase their uptake by GRP receptors. Average uptake in the pancreas for the starch-bombesin nanoparticles system appears dramatically higher than in the passively targeted nanoparticle case, however the large uptake is due primarily to one mouse

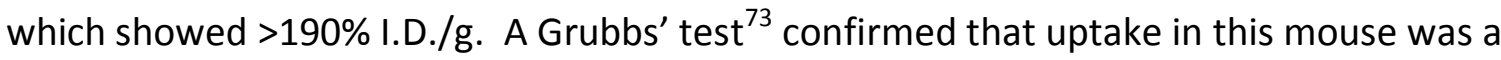
statistical outlier, and results for this mouse were disregarded. After elimination of the outlier, the biodistribution data more closely resembles that of gum arabic nanoparticles than ${ }^{64} \mathrm{Cu}$-bombesin conjugates. The biodistribution data of untargeted starch capped nanoparticles and starch-bombesin capped nanoparticles (after elimination of the outlier) are shown in Figure 2.5.5 with and without blocking with unconjugated bombesin peptide. Differences between the four groups showed no statistical significance after removal of the outlier.

Previous reports indicated that starch-bombesin capped gold nanoparticles exhibited specific, receptor-mediated uptake both in vitro and in vivo. ${ }^{74}$ In vitro, the nanoparticles show variable $\mathrm{IC}_{50}$ values against GRP receptor, from $8.10 \pm 0.30 \mu \mathrm{g} / \mathrm{mL}$ for low bombesin loading to $2.45 \pm 0.18 \mu \mathrm{g} / \mathrm{mL}$ for high bombesin loading. ${ }^{71}$ The study 
did not include controls of starch-coated gold nanoparticles, therefore no conclusions can be made about the specificity of the nanoparticle system despite its high affinity for the GRP receptors.

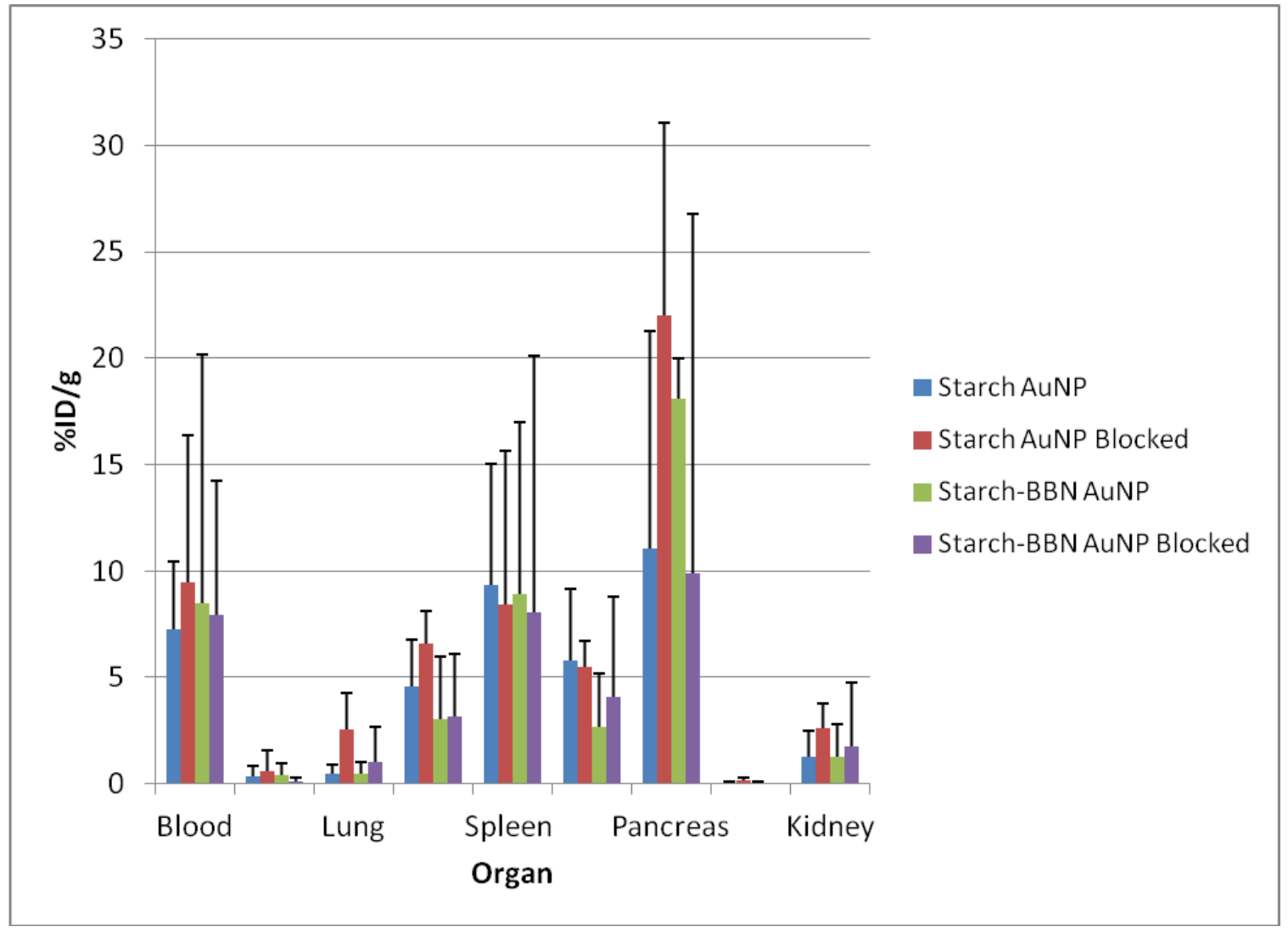

Figure 2.5.5: Biodistribution of starch capped gold nanoparticles. These nanoparticles show biodistribution and clearance through the RES system. Actively targeted starchbombesin capped nanoparticles show no significantly different uptake in vivo.

Error bars in the in vivo experiments show wide variability in pancreatic uptake consistent with a single outlier that was not excluded. While the ${ }^{64} \mathrm{Cu}$-bombesin conjugate confirms that bombesin can be an effective targeting agent for the GRP 
receptor, bombesin was ineffective in the starch-capped gold nanoparticle system.

While there is variance in starch size based on the plant source, potato starches tend to consist of a 24-30 glucose oligomer chain. ${ }^{75}$ Given the large size of the nanoparticle surfactant and the small size of the linker (a 5 carbon chain from lipoic acid), the targeting portion of bombesin may have been covered by the starch and not exposed to GRP receptors in vivo. Lipoic acid-bombesin conjugates do not fully displace the starch surfactant, but merely attach to the surface displacing a portion of the attached starch. The remaining starch molecules may sterically hinder bombesin-GRP receptor interactions, blocking the specific targeting. Additionally, the amino acids of the bombesin peptide may interact with the starch molecules themselves, further decreasing the amount of targeting sequences accessible to GRP receptors. It is unlikely that the bombesin separates from the nanoparticles as the nanoparticles do not revert to their pre-conjugation solubility. Additionally, a change in the UV-Vis absorbance would appear after dissociation, which is not observed. Another potential explanation for the poor in vivo targeting is rapid clearance of the nanoparticles through the RES. The bombesin conjugates do not circulate for a sufficient time to bind to the GRP receptor, which would explain the discrepancy between the high in vitro binding and the low in vivo binding.

Future experiments should focus on the effect of different bombesin conjugation approaches on GRP receptor uptake. Longer polyethylene glycol (PEG) linkers are available from a variety of sources, and can easily be linked to the peptide. Other, less 
bulky and easily displaced surfactants are also available. The citrate reduction of the Turkevich procedure creates biocompatible nanoparticles that can be further functionalized with lipoic acid linkages. Citrate-capped nanoparticles are unstable towards aggregation in vivo, however, they can be passivated by addition of a PEG

group linked to lipoic acid. ${ }^{76}$ An alternative system would utilize a citrate capped gold nanoparticle linked to a commercially available lipoic acid-PEG-COOH linker. The bombesin peptide would then be attached via 1-Ethyl-3-(3dimethylaminopropyl)carbodiimide (EDC)/sulfo N-hydroxy succinimide (NHS) chemistry. $^{49}$

\section{$2.6{ }^{64} \mathrm{Cu}$-DTDTPA Gold Nanoparticle Experimental}

\subsubsection{Materials and Measurements}

All chemicals were at least ACS grade and purchased from Sigma-Aldrich and used as received unless otherwise noted. High purity $(>18 \mathrm{M} \Omega)$ water was produced from an in-house generator. TEM images were obtained on a JEOL 1400 transmission electron microscope (JEOL Ltd, Tokyo, Japan). TEM samples were prepared by placing 5 $\mu \mathrm{L}$ of gold nanoparticle solution on a 300 mesh carbon-coated copper grid and allowing the solution to stand for five minutes. Excess solution was removed carefully and the grid was allowed to dry for an additional five minutes. The average size and distribution of the gold nanoparticles synthesized were determined by the processing of the TEM 
image using Adobe Photoshop (with Fovea plugins). The hydrodynamic diameter and the zeta potential were obtained using a Zetasizer Nano S90 (Malvern Instruments Ltd. USA) using a dilute $(0.08 \mathrm{~g} / \mathrm{L})$ aqueous solution. The UV-Vis absorption spectra were recorded at room temperature using Varian Cary 50 UV/Vis spectrophotometers. The absorption measurements were performed on dilute colloidal gold nanoparticle solution in disposable cuvettes with a $10 \mathrm{~mm}$ path length. Estimation of gold concentration in conjugates was performed using furnace atomic absorption spectroscopy (AAS) with a standard curve spanning 0-40 $\mu \mathrm{g} / \mathrm{L}$ (Perkin-Elmer AAnalyst 800 ThGA graphite furnace). Quality-control materials (duplicates, spikes, and instrument-calibration verification) were within appropriate ranges. Dithiolated diethylene triamine pentacetic acid (DTDTPA) and nanoparticle synthesis, characterization, and cell studies were performed by Dr. Ajit Zambre (sections 2.6.2-2.6.5 and 2.6.9). Radiolabeling, radiostability, radiosynthesis, and data analysis for animal studies were performed by Mark McLaughlin (sections 2.6.6-2.6.8). Biodistribution and animal studies were performed by Lisa Watkinson and Terry Carmack under the supervision of Prof. John Lever and Dr. Jeff Smith at the Harry S Truman Memorial Veterans Affairs Hospital (section 2.6.10).

\subsubsection{DTDTPA Synthesis}

DTPA $(0.78 \mathrm{~g})$, acetic anhydride $(0.75 \mathrm{~mL})$ and anhydrous pyridine $(1.02 \mathrm{~mL})$ were mixed and heated to $70^{\circ} \mathrm{C}$ for $18 \mathrm{hrs}$ in a $5 \mathrm{~mL}$ glass V-bottom vial. The resultant mixture was cooled to room temperature and further chilled to $4^{\circ} \mathrm{C}$. The precipitate 
was collected by filtration, purified by repeated washing with acetic anhydride followed by anhydrous ether. The washed precipitate was filtered and dried under vacuum to obtain $0.63 \mathrm{~g}, 91 \%$ of DTPA-bisanhydride (DTPA-BA). ${ }^{77}$

DTPA-BA $\left(0.5 \mathrm{~g}, 5.6 \times 10^{-3} \mathrm{~mol}\right)$ was dissolved in $10 \mathrm{~mL}$ of dimethylformamide (DMF) in a $100 \mathrm{~mL}$ round bottom flask with constant heating at $70^{\circ} \mathrm{C}$. Aminoethanethiol $\left(0.25 \mathrm{~g}, 1.23 \times 10^{-2} \mathrm{~mol}\right)$ was dissolved separately in another flask with $8 \mathrm{~mL}$ of DMF and $0.44 \mathrm{~mL}$ of triethylamine. This solution was then added to the round bottom flask containing DTPA-BA with constant stirring and the reaction mixture was heated to $70{ }^{\circ} \mathrm{C}$ overnight. Subsequently, the reaction mixture was cooled to room temperature and placed in an ice bath. The resulting white residue (triethylamine hydrochloride) was filtered. The filtrate was then concentrated at reduced pressure and further mixed with chloroform to form a white precipitate. This precipitate was filtered and washed thoroughly with chloroform to remove impurities. The precipitate was dried under vacuum to obtain white DTDTPA $(0.49 \mathrm{~g}, 86 \%)$ and characterized by nuclear magnetic resonance $(\mathrm{NMR}) .^{78}$

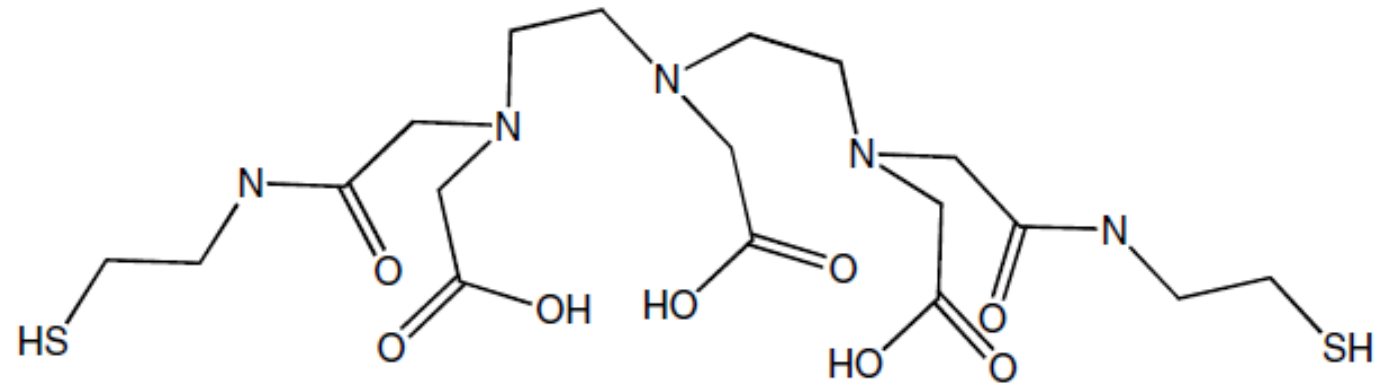

Figure 2.6.1: Structure of DTDTPA. 


\subsubsection{Synthesis of DTDTPA Gold Nanoparticle Conjugates}

DTDTPA functionalized nanoparticles were prepared by dissolving $200 \mathrm{mg}$ (5.1 x $10^{-5} \mathrm{~mol}$ ) of $\mathrm{HAuCl}_{4} \cdot 3 \mathrm{H}_{2} \mathrm{O}$ in $120 \mathrm{~mL}$ of methanol in a $500 \mathrm{~mL}$ round bottom flask. In a separate flask, $482 \mathrm{mg}\left(9.4 \times 10^{-5} \mathrm{~mol}\right)$ of DTDTPA was dissolved in $40 \mathrm{~mL}$ of methanol and $2 \mathrm{~mL}$ of glacial acetic acid. This solution was added with continuous stirring to an aqueous solution of gold salt to produce an orange solution. To this mixture, $190 \mathrm{mg}$ (5 $\mathrm{mmol}$ ) of $\mathrm{NaBH}_{4}$ dissolved in $14 \mathrm{~mL}$ of water was added under vigorous stirring at room temperature. Immediately after addition of $\mathrm{NaBH}_{4}$, the solution became dark brown followed by appearance of black flocculate. The resultant mixture was allowed to stir for 1 hour at room temperature before addition of $5 \mathrm{~mL}$ of $1 \mathrm{M} \mathrm{HCl}$. This black solution of gold nanoparticles was then centrifuged at 7000 RPM for 20 minutes. The supernatant was removed and the particles were washed twice with $0.01 \mathrm{M} \mathrm{HCl}$, using the same centrifugation parameters. The particles were further washed successively with water followed by diethyl ether. The resulting black powder of Au nanoparticleDTDTPA was dried under vacuum and stored at $-20^{\circ} \mathrm{C}$. As needed, the particles were readily dispersed in $0.01 \mathrm{M} \mathrm{NaOH}$ and were stable against aggregation for over a month as determined by UV-Vis spectroscopy.

\subsubsection{Chelation of $\mathrm{Cu}^{2+}$ with Gold-DTDTPA Nanoparticles}

Copper was added to Au-DTDTPA at room temperature with continuous stirring. A $5 \mathrm{~mL}$ solution of $10 \mathrm{mg} / \mathrm{mL}$ Au-DTDTPA was prepared by dissolving nanoparticles in 5 
$\mathrm{mL}$ of $1 \mathrm{M} \mathrm{NaOH}$, then neutralized to $\mathrm{pH}=7.0$ with $1 \mathrm{M} \mathrm{HCl}$. Ten $\mathrm{mg}$ of $\mathrm{CuCl}_{2}$ was added to this solution with continuous stirring. The formation of precipitate was immediately observed. The reaction mixture was stirred for 5 minutes to produce a brownish black residue which was collected by centrifugation. The residue was washed with deionized (DI) water thoroughly to remove any unreacted copper salt and dried under vacuum. The amount of copper required to saturate the chelate was monitored by titrimetric analysis utilizing atomic absorption spectroscopy.

\subsubsection{Titrimetric Analysis of Copper on Gold-DTDTPA Nanoparticles:}

The amount of $\mathrm{Cu}^{2+}$ ions required for complete saturation of Au-DTDTPA was determined by AAS measurements. The titration was performed by successive addition of an aqueous solution of $\mathrm{CuCl}_{2}(2 \mathrm{mg}-30 \mathrm{mg})$ to $10 \mathrm{mg}$ of colloidal Au-DTDTPA dispersion at room temperature with continuous stirring. After the reaction was complete the reaction mixtures were collected in individual centrifuge tubes and centrifuged at 5000 RPM for 5 minutes. The pellets were resuspended in DI water, centrifuged, and the supernatants decanted. All the supernatants and pellets were analyzed for copper content by AAS.

\subsection{6 ${ }^{64} \mathrm{Cu}$ Isotope Production and Specific Activity Analysis}

Targets were irradiated as previously described ${ }^{79}$ by Tom Brossard using approximately $12 \mathrm{MeV}$ protons on a GE PETtrace cyclotron at MURR with a 99\%

enriched ${ }^{64} \mathrm{Ni}$ target. Isotope yield measurements were determined using either a $\gamma$ 
spectrometer (Canberra model 1510, Meriden, CT) and/or a radioisotope dose calibrator (Capintec CRC-10, Pittsburgh, PA). The $y$ spectrometer was used to detect radionuclidic impurities. Specific activities were determined as described previously for

${ }^{64} \mathrm{Cu}$ by titration of ${ }^{64} \mathrm{CuCl}_{2}$ with the macrocyclic chelator TETA. ${ }^{79}$ Briefly, aliquots of varying amounts of TETA were added to ${ }^{64} \mathrm{Cu}$ and the complexation yield monitored by radio-TLC. The samples were spotted on C-18 plates and the plates developed using 1:1 methanol: $10 \%$ ammonium acetate with two drops of concentrated $\mathrm{HCl}$. $\mathrm{CuCl}_{2}$ remains at the origin whereas complexed copper in the form of $\mathrm{Cu}(T E T A)^{2-}$ migrated with $\mathrm{R}_{f}=$ 0.9. The minimum TETA concentration for which $100 \%$ labeling occurred was assumed to be equal to the concentration of $\mathrm{Cu}^{+2}$ present.

\subsubsection{Radiochemical Synthesis of ${ }^{64} \mathrm{Cu}$ Gold-DTDTPA Nanoparticles}

Five $\mathrm{mg}$ of Au-DTDTPA was dissolved in $500 \mu \mathrm{L}$ of $1 \mathrm{M} \mathrm{NaOH}$ to give a $10 \mathrm{mg} / \mathrm{mL}$ solution. This solution $(20 \mu \mathrm{L})$ was added to $750 \mu \mathrm{L}$ of a buffer solution consisting of 0.4 $\mathrm{M}$ ammonium acetate adjusted to $\mathrm{pH}=4$ using $\mathrm{HCl}$ and sonicated for 30 minutes. Radioactive ${ }^{64} \mathrm{CuCl}_{2}$ (MURR cyclotron facility, $81 \mathrm{MBq}$ ) was received in $150 \mu \mathrm{L}$ of $0.1 \mathrm{M}$ $\mathrm{HCl}$. Forty-five $\mu \mathrm{L}$ of ${ }^{64} \mathrm{CuCl}_{2}(27 \mathrm{MBq})$ was added to the Au-DTDTPA/buffer solution and placed in a boiling water bath for 30 minutes. Unlabeled copper was removed from solution by eluting the labeled nanoparticles through a chelex 100 cation exchange resin (Bio Rad) column with $18 \mathrm{M} \Omega$ water. The column was prepared by dispersing $300 \mathrm{mg}$ of resin in $7 \mathrm{~mL}$ of water and depositing the slurry in a Bio Rad Poly-Prep $9 \mathrm{~cm}$ column. 
Excess liquid was allowed to pass through the slurry and the prepared column was then washed twice with $2 \mathrm{~mL}$ of PBS. The nanoparticle solution $(1.25 \mathrm{~mL})$ was loaded onto the column and eluted with $5 \mathrm{~mL}$ of PBS. The effluent and column were counted on a $\gamma$ spectrometer using the $511 \mathrm{keV}$ peak to determine labeling yield. Nanoparticle solution was adjusted to $\mathrm{pH}=7.5$ using $\mathrm{NaOH}$ and $\mathrm{HCl}$, then diluted to a volume of $2.5 \mathrm{~mL}$ using PBS.

\subsubsection{In Vitro Stability of Gold-DTDTPA and ${ }^{64} \mathrm{Cu}$ Gold-DTDTPA Nanoparticles}

In vitro stability studies of ${ }^{64} \mathrm{Cu}$-Au-DTDTPA were performed by challenging 0.5 $\mathrm{mL}$ of ${ }^{64} \mathrm{Cu}$-Au-DTDTPA with water, PBS, cysteine, or rat serum albumin (RSA). Nanoparticle conjugates were synthesized as in 2.6 .7 using $5 \mu \mathrm{L}$ of $10 \mathrm{mg} / \mathrm{mL}$ nanoparticles in $495 \mu \mathrm{L}$ of water. Each of these solutions then received $\sim 17 \mathrm{MBq}$ of ${ }^{64} \mathrm{CuCl}_{2}$. Addition of $500 \mathrm{~mL}$ of water, $2 \times \mathrm{PBS}, 0.2 \mathrm{M}$ cysteine, or $0.2 \mathrm{M}$ RSA gave final concentrations in each vial of PBS or $0.1 \mathrm{M}$. The stability was measured by monitoring the UV-visible absorbance and radio-TLC at 0,24 , and 48 , and 120 hours. Radio-TLC was performed with C18 plates (Whatman) in a developing solution of 1:1 by volume solution of methanol:10\% ammonium acetate.

\subsubsection{In Vitro Cytotoxicity Measurements}

In vitro cytotoxicity was evaluated using a 3-(4,5-Dimethylthiazol-2-yl)-2,5diphenyltetrazolium bromide (MTT) cell proliferation assay kit (Promega Corporation). Cells at the exponential growth phase $\left(1 \times 10^{5}\right.$ cells $\left./ \mathrm{mL}\right)$ were placed in a flat-bottom 96 - 
well polystyrene-coated plate and incubated in a $\mathrm{CO}_{2}$ incubator at $5 \% \mathrm{CO}_{2}$ and $37^{\circ} \mathrm{C}$ for 24 hours. A series of concentrations $(0,1,2.5,5,10,20$ and $40 \mu \mathrm{g} / \mathrm{mL})$ of Au-DTDTPA and $\mathrm{Cu}$-Au-DTDTPA were added to wells in quadruplet. After incubation for an additional 24 hours, $10 \mu \mathrm{L}$ MTT ( $5 \mathrm{mg} / \mathrm{mL}$ in PBS) was added to each well and kept for 24 hours. Formazan crystals produced by living cells were dissolved in $100 \mu \mathrm{L}$ of isopropanol added to each well plate and kept in the dark at $25^{\circ} \mathrm{C}$ for 18 hours. The intensity of developed color was measured by micro plate reader (Dynastic MR 5000) operating at $570 \mathrm{~nm}$ wavelength. Wells with complete medium, nanoparticles, and MTT, but without cells, were used as blanks. Untreated cells were considered $100 \%$ viable.

\subsubsection{In Vivo Biodistribution Measurements}

Biodistribution and animal studies were performed by Lisa Watkinson and Terry Carmack under the supervision of Prof. John Lever and Dr. Jeff Smith at the Harry S Truman Memorial Veterans Affairs Hospital. Evaluation of pharmacokinetics of ${ }^{64} \mathrm{Cu}-\mathrm{Au}$ DTDTPA was conducted in normal CF1 mice. Nanoparticle conjugates (1.48 MBq in 100 $\mu L$ of PBS) were injected via the tail vein $(n=3)$. Animals were sacrificed at $0.25,0.5,2,4$, and 24 hours post injection by cervical dislocation. The following organs were collected for measurement on a well counter: tail, blood, heart, lung, liver, spleen, stomach, large intestine, small intestine, kidney, urine, feces, muscle, bone, bladder, brain, pancreas, and carcass. 


\section{$2 .{ }^{64} \mathrm{Cu}$ Gold-DTDTPA Results and Discussion}

Au-DTDTPA nanoparticles fail to show an absorption maximum associated with the plasmon resonance. The spectrum shows a small absorption hump around $525 \mathrm{~nm}$ that corresponds with the expected plasmon resonance peak in both the Au-DTDTPA nanoparticles and the Cu-Au-DTDTPA nanoparticles. These absorption spectra are shown in Figure 2.7.1. ${ }^{80}$
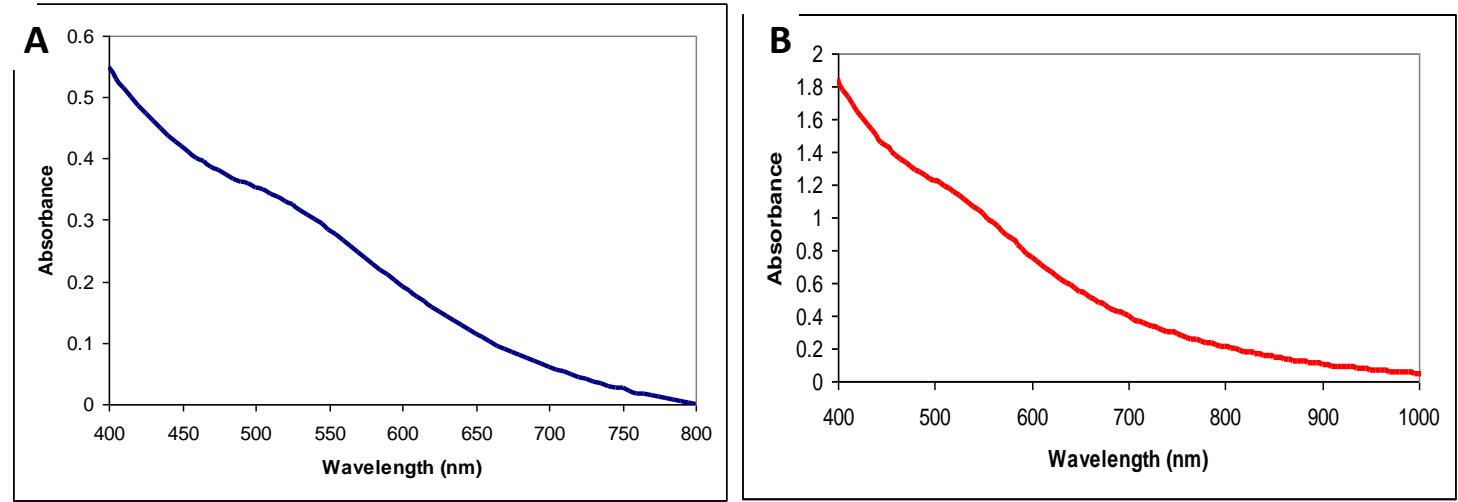

Figure 2.7.1 A and B: UV-Vis absorption spectra of Au-DTDTPA (A) and Cu-Au-DTDTPA (B).

TEM analysis shows that the nanoparticles have a metallic diameter of $\sim 3 \mathrm{~nm}$.

Some variability is present, with nanoparticles as large as $20 \mathrm{~nm}$ in diameter. This size is typical of nanoparticles made via the Brust procedure. ${ }^{28}$ Figure 2.7.2 shows a characteristic TEM of the nanoparticles. 


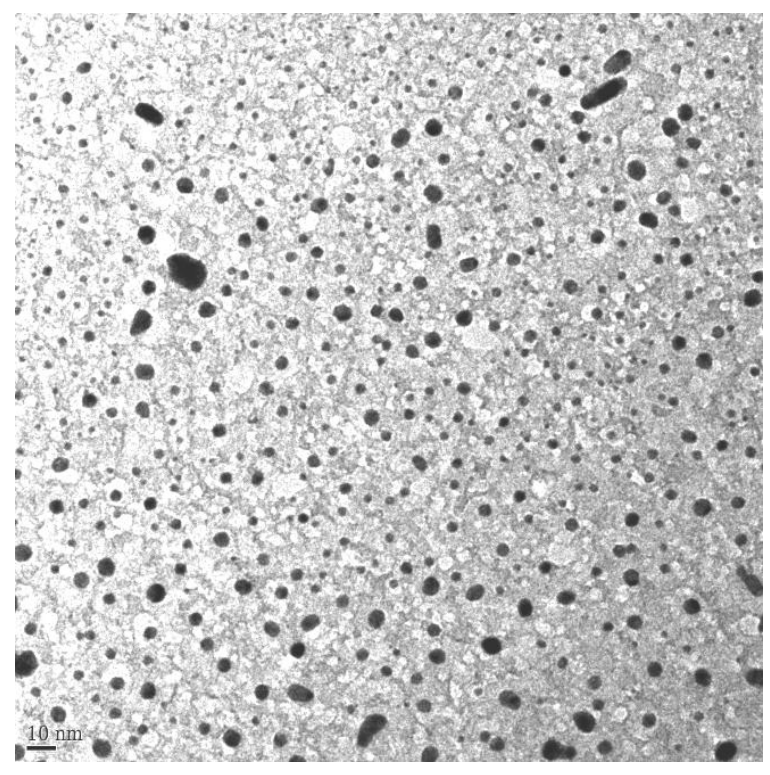

Figure 2.7.2: TEM showing a characteristic Au-DTDTPA nanoparticle dispersion

Hydrodynamic diameter and zeta potential are shown in Table 2.7.1. The large hydrodynamic diameter relative to the small metallic diameter is also expected. Zeta potential values that are highly positive or negative indicate the nanoparticles should be stable towards aggregation. ${ }^{81}$ The saturation curve as measured by AAS is shown in Figure 2.7.3. At low levels of added copper, synthetic yield is constant. Addition of more than $4.72 \mathrm{mg}$ of copper saturates the $10 \mathrm{mg}$ of Au-DTDTPA used and leads to decreasing yield. This saturation was confirmed using radio-TLC and ${ }^{64} \mathrm{Cu}$.

Table 2.7.1: DLS properties of Au-DTDTPA and Cu-Au-DTDTPA

\begin{tabular}{|c|c|c|}
\hline Construct & Hydrodynamic Diameter $(\mathbf{n m})$ & Zeta Potential $(\mathbf{m V})$ \\
\hline Au-DTDTPA & 133.6 & -40.63 \\
\hline Cu-Au-DTDTPA & 149.3 & -55.06 \\
\hline
\end{tabular}




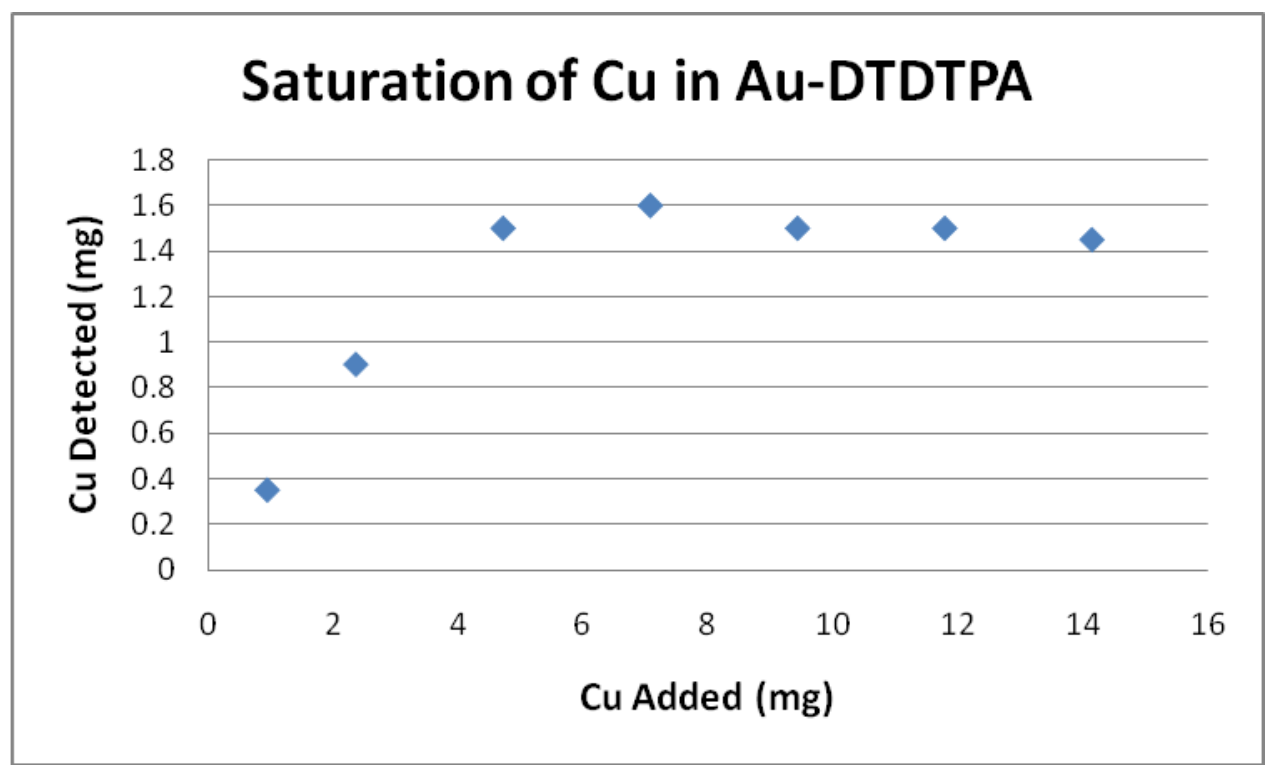

Figure 2.7.3: Copper saturation of Au-DTDTPA as measured by AAS

Cu-Au-DTDTPA nanoparticles monitored in water, PBS, and rat serum show constant plasmon resonance peak location and intensity as monitored by UV-Vis spectrometry. The constant peak indicates that the nanoparticles are stable towards aggregation. Nanoparticles monitored in cysteine show a consistent wavelength of maximum absorption, but decreased absorption intensity over the monitoring period. The peak completely disappears by 120 hours. Cysteine contains a sulfur side chain, which can displace the thiolated DTDTPA chelating agent from the surface of the gold nanoparticle. Without the protection provided by the DTDTPA ligand, the nanoparticles likely aggregate and the plasmon resonance peak disappears. Figure 2.7.4 shows the decrease of the Cu-Au-DTDTPA plasmon resonance over time. 


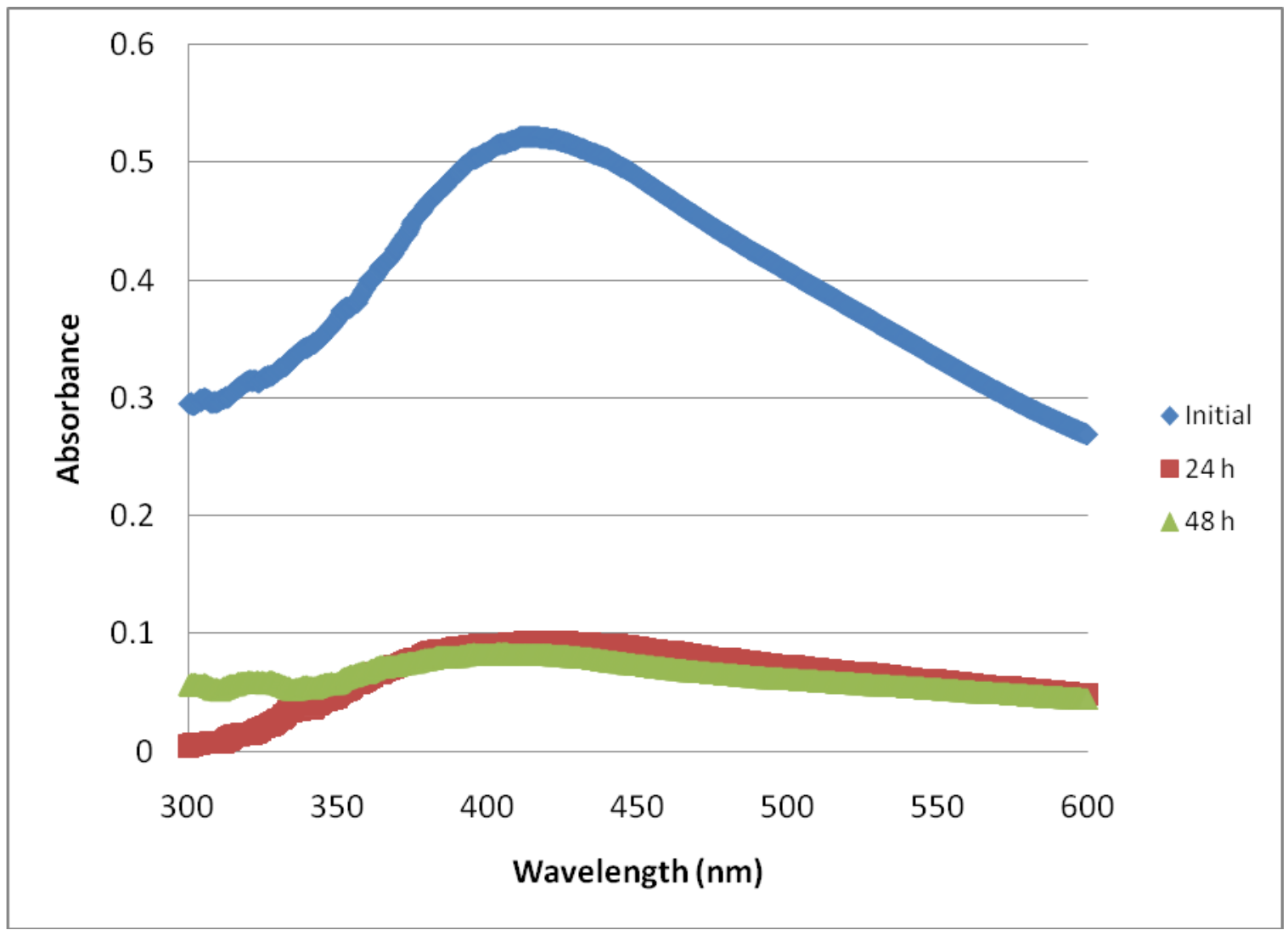

Figure 2.7.4: Stability of Cu-Au-DTDTPA in cysteine. UV-Vis spectroscopy of Cu-Aushows decrease in plasmon resonance over time, ultimately disappearing at 120 hours.

Radio-TLC measurements indicate the same process. Initially, radio-TLC shows one large peak at the origin for Cu-Au-DTDTPA samples in each biological mimic solution. Over time, an increasing amount of activity is seen in the cysteine solution at an $R_{f}$ of 0.2 , indicating that the activity is being displaced from the gold nanoparticle. After 96 hours, roughly half of the activity has been displaced from the particle surface. Because of the stability of the Cu-DTDTPA complex, we conclude that the loss of activity is indicative of the loss of chelate from the Au nanoparticles. Figures 2.7.5 A and B show 
a characteristic radio-TLC and the cysteine solution radio-TLC after 96 hours, respectively.
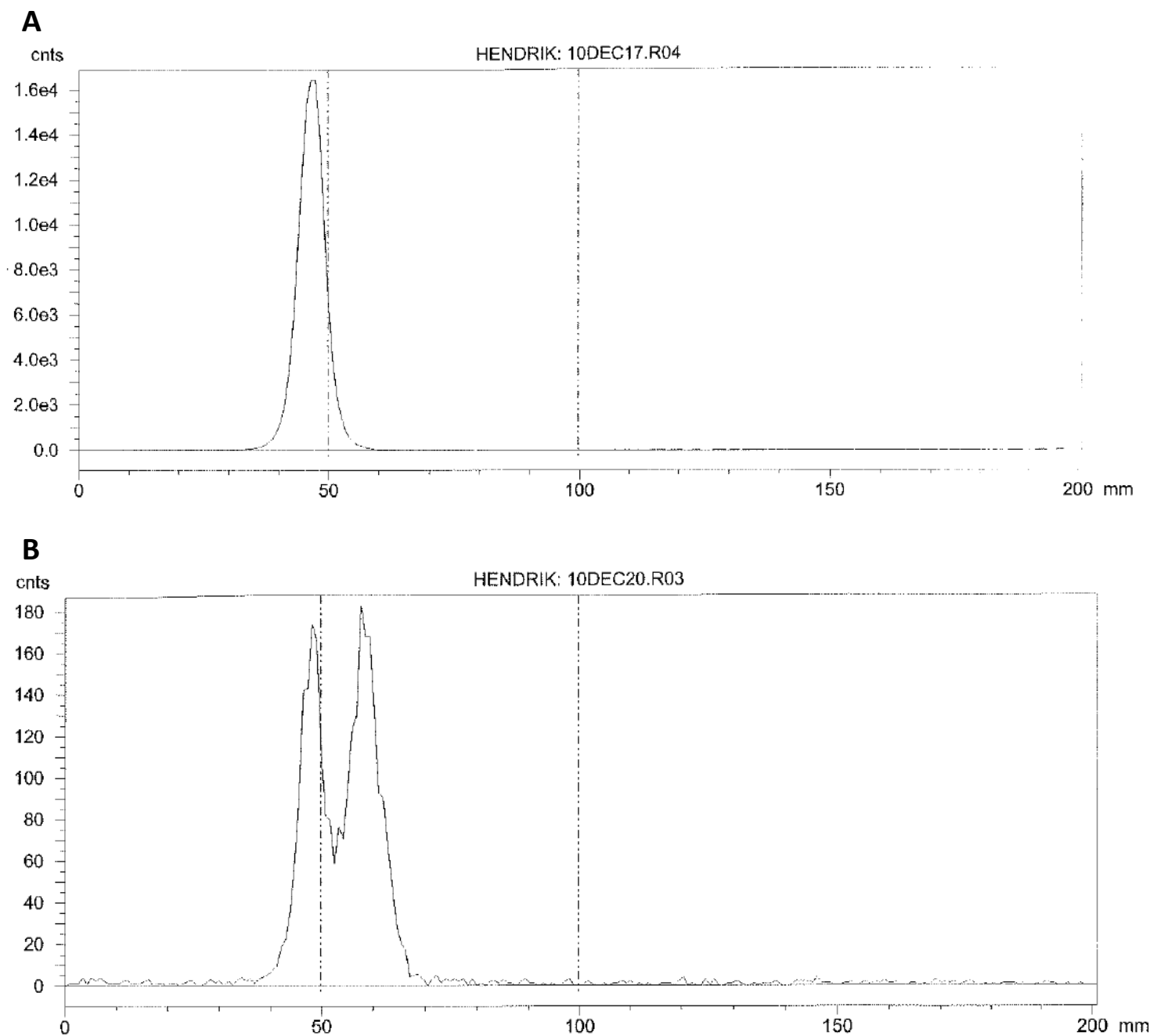

Figure 2.7.5 A and B: Stability studies of ${ }^{64} \mathrm{Cu}-\mathrm{Au}-\mathrm{DTDTPA}$ via radio-TLC. These chromatograms are characteristic of ${ }^{64} \mathrm{Cu}-\mathrm{Au}-\mathrm{DTDTPA}$ in water/PBS/rat serum (A) and after 96 hours in cysteine (B). Activity travels up the TLC plate as the chelate is displaced from the nanoparticles. The origin is at $50 \mathrm{~mm}$ and the solvent front at $100 \mathrm{~mm}$. 
While the instability in cysteine solution is troubling, significant dissociation only occurs at high concentrations of cysteine and after multiple half-lives of ${ }^{64} \mathrm{Cu}$. Mammalian levels of cysteine typically vary from $20-100 \mu \mathrm{M}^{82}$ whereas the nanoparticle conjugate was challenged with a solution of $0.2 \mathrm{M}$ cysteine. After 8 half-lives (96 hours), $50 \%$ of the activity remains bound to the gold nanoparticles. While an ideal construct would exhibit no dissociation, the nanoparticles are likely stable enough to warrant further study. First, the concentration of cysteine used to test stability is over 1,000 times higher than what is observed in vivo. Lower concentrations of cysteine will likely cause less significant dissociation. As imaging times with ${ }^{64} \mathrm{Cu}$ are often on the order of 24-48 hours, ${ }^{83}$ the stability of our complex after 48 hours warrants further study.

In vitro cytotoxicity measurements with $\mathrm{SKBr} 3$ breast cancer cells indicate that the Au-DTDTPA nanoparticles are well tolerated at low concentrations. However, above $10 \mu \mathrm{g} / \mathrm{mL}$, cells showed decreased survivability relative to controls. Figure 2.7.6 shows the effect of Au-DTDTPA and Cu-Au-DTDTPA on breast cancer cells. 


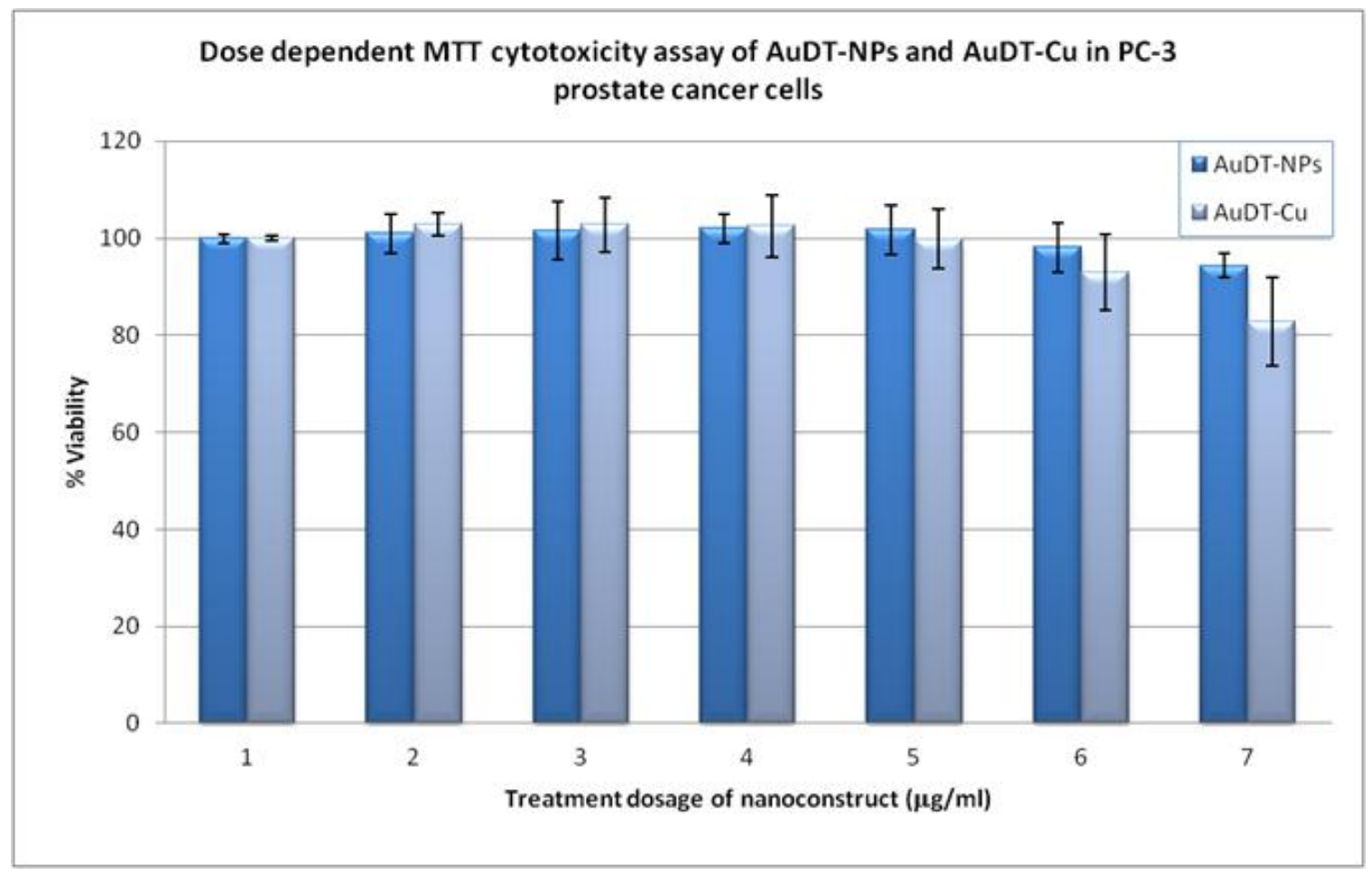

Figure 2.7.6: Cell viability studies of breast cancer SKBr3 cells

Nanoparticles prepared for biodistribution studies were labeled with a chemical yield of $94 \%$ as determined by activity load on the cation exchange column. Unbound ${ }^{64} \mathrm{CuCl}_{2}$ was completely retained on the column. In vivo, the ${ }^{64} \mathrm{Cu}-\mathrm{Au}-\mathrm{DTDTPA}$ showed uptake consistent with passively targeted nanoparticle constructs. Initially, activity appears primarily in the liver, then decreases as the nanoparticles are cleared through the RES or hepatobiliary system and are excreted in the feces. Approximately $1 / 3$ of the injected activity clears through the feces after 24 hours. Activity present in the kidney likely represents ${ }^{64} \mathrm{Cu}$-DTDTPA, as the nanoparticle construct itself is likely too large to enter the glomerular filtrate and over $95 \%$ of free ${ }^{64} \mathrm{Cu}$ binds proteins found in serum. ${ }^{84}$ 

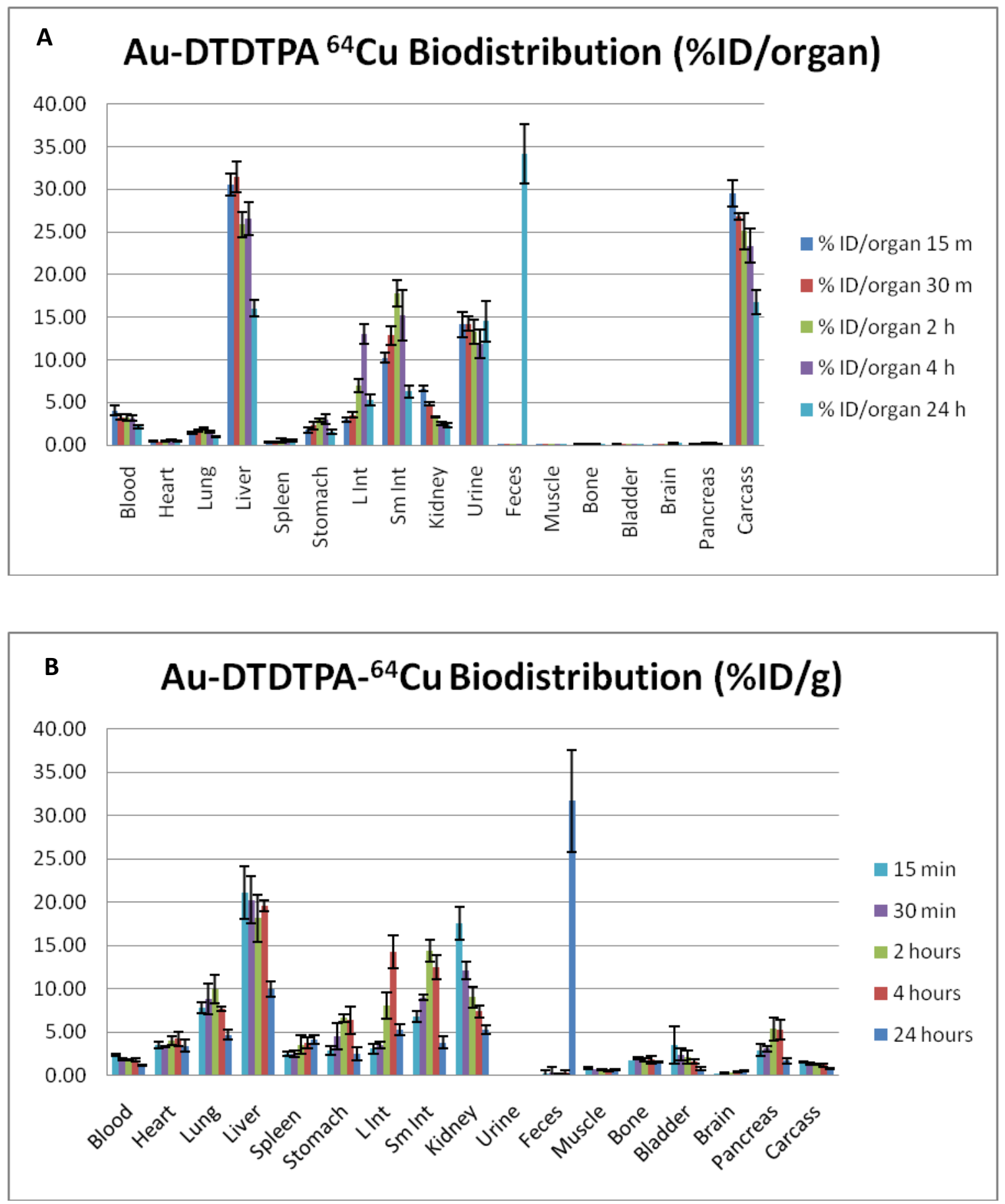

Figures 2.7.7 A and B: Uptake of ${ }^{64} \mathrm{Cu}$-Au-DTDTPA nanoparticles as \%ID/organ (A) and \%ID/g (B). Biodistribution shows uptake similar to untargeted, gum arabic coated nanoparticles. 
Additionally, the negative charge on the nanoparticle further limits filtration and urine excretion. ${ }^{85}$ Figures 2.7.7 A and B show uptake as \%ID/organ and \%ID/g, respectively.

Stability of the ${ }^{64} \mathrm{Cu}$-Au-DTDTPA nanoparticle system under the observed conditions is inconclusive. While nanoparticles are expected to clear via the RES and hepatobiliary system, free ${ }^{64} \mathrm{Cu}$ also clears via the hepatobiliary system with excretion in the feces after 24 hours. ${ }^{86}$ The large uptake in the liver could indicate uptake through either of these routes. Uptake in the spleen, primarily due to RES clearance, is lower than in the case of the passively targeted gum arabic gold nanoparticles. This difference could be explained by a number of factors, including partial dissociation of ${ }^{64} \mathrm{Cu}$ from DTPA or differences in nanoparticle surface charge, size, or hydrophilicity.

A dual label experiment would conclusively determine the stability of the ${ }^{64} \mathrm{Cu}$ DTDTPA-nanoparticle conjugate. Comparing the biodistribution of gold nanoparticles synthesized from ${ }^{198 / 199} \mathrm{Au}$ and the attached ${ }^{64} \mathrm{Cu}$-DTDTPA would establish the viability of the system. Similar biodistributions for ${ }^{64} \mathrm{Cu}$ and ${ }^{198 / 199} \mathrm{Au}$ indicate that the conjugate remains largely intact while significantly different biodistributions indicate that the ${ }^{64} \mathrm{Cu}$ does not remain attached to the nanoparticle in vivo. If the system is stable, it would warrant future study. The small size of the nanoparticles indicates they may show high tumor uptake via the EPR effect. ${ }^{87}$ Finally, specific targeting agents such as bombesin could be added to target a particular type of tumor rather than relying on passive uptake. 


\section{CHAPTER 3:}

\section{GOLD SHELL NANOPARTICLES FOR in vivo ${ }^{225}$ Ac $\alpha$-GENERATOR}

\subsection{Chemistry of ${ }^{225} \mathrm{Ac}$ and the Lanthanides}

Chemical interest in the actinides focuses primarily on their wide variety of oxidation states and unique f-orbital interactions. These properties of actinides give them a wide range of colors and facilitate their use as catalysts. ${ }^{88}$ Despite also filling $\mathrm{f}$ orbitals, the lanthanide series shows much less oxidation state variety. All of the lanthanides primarily form the $3+$ oxidation state, with the exception of $\mathrm{Ce}$, which also easily forms the $4+$ state $^{89}$ As the first actinide, the electronic structure of Ac does not heavily populate the $5 f$ orbital. Because of this, its chemical behavior more closely resembles the lanthanides rather than the rest of the actinide series. Actinium easily loses three electrons to form the $3+$ oxidation state.

Additionally, relativistic effects further contribute to the similarity between Ac and the lanthanides, in particular the early lanthanides. Due to relativistic effects, the atomic radii of $\mathrm{Ac}$ (in particular the s shells) are more contracted than what would otherwise be expected. Therefore, the difference in size between Ac and La is smaller 
than indicated by non-relativistic size calculations. These relativistic effects are also responsible for the surprising stability of high oxidation states found in many of the actinides. $^{90}$

Previous studies incorporated ${ }^{225} \mathrm{Ac}$ into $\mathrm{LaPO}_{4}$ nanoparticles by a process of precipitation from organic solution. Phosphates of the lanthanides are highly insoluble

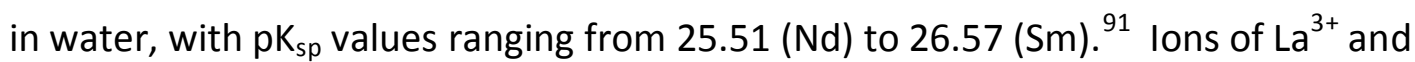
${ }^{225} \mathrm{Ac}^{3+}$ mixed with phosphoric acid produce a precipitation of monodisperse nanoparticles with $44 \%$ radiochemical yield. This particle system is capable of retaining $\sim 50 \%$ of the ${ }^{221} \mathrm{Fr}$ daughter and $99.9 \%$ of the ${ }^{225} \mathrm{Ac}$ parent. ${ }^{48}$ Conjugation to biological targeting agents occurs via an ionic linkage between 6-aminohexanoic acid and surface

phosphate. Unfortunately, the complex synthesis involves inert atmosphere conditions and reproducibility issues. Presumably, the ionic linkage remains stable in the aqueous in vivo environment due to the association of residual organic compounds with the surface of the nanoparticle. An aqueous synthetic pathway, which could reliably be used under normal atmospheric conditions, would be desirable.

\subsection{Nanoparticle Synthesis and Characterization Experimental}

\subsubsection{Materials and Equipment}

All chemicals were used as received from Sigma-Aldrich unless otherwise noted and were ACS grade or higher. Water used was purified using an in house purification 
system to $18 \mathrm{M} \Omega$ or higher. Radioactivity measurements were performed with a $\gamma$ spectrometer consisting of a high purity germanium detector and a Canberra Industries multichannel analyzer. Full width at half maximum resolution of the detector was 0.7 $\mathrm{keV}$ at $218.2 \mathrm{keV}$. Detector dead times were typically $<1 \%$ and count rates were automatically corrected for the detector dead time by the $\gamma$ spectroscopy software. A regenerated cellulose dialysis membrane with a $10 \mathrm{kDa}$ molecular weight cutoff (MWCO, Spectra/Por, 128106) was used to purify nanoparticle dispersions. The dialysis membrane was washed of preservatives with $18 \mathrm{M} \Omega$ water before dialysis against 18 $\mathrm{M} \Omega$ water. A $0.4 \mathrm{~T}$ NdFeB magnet (Supermagnet \#31, United Nuclear) was used for magnetic separation of Gd containing nanoparticles from solution. Nanoparticles were characterized by TEM (JEOL 1400), NAA, x-ray diffraction (XRD, Scintag X2), and electron energy loss spectroscopy-TEM (EELS-TEM, Zeiss Libra 120). All ${ }^{225}$ Ac processes were performed at Oak Ridge National Laboratory.

\subsubsection{Purification of ${ }^{225} \mathrm{AcCl}_{3}$}

Actinium-225 chloride was prepared as previously described from a stock of

${ }^{229}$ Th. $^{92}$ Briefly, ${ }^{225} \mathrm{Ra}$ and ${ }^{225} \mathrm{Ac}$ are separated from the parent ${ }^{229} \mathrm{Th}$ using two columns of MP1 (Bio-Rad Laboratories) anion exchange resin in sequence with $8 \mathrm{M} \mathrm{HNO}_{3}$. The ${ }^{225} \mathrm{Ac}$ is further separated from ${ }^{225} \mathrm{Ra}$ using two sequential columns of AG50-X4 cation exchange resin. Radium was eluted with $1.2 \mathrm{M} \mathrm{HNO}_{3}$ while ${ }^{225} \mathrm{Ac}$ was eluted with $8 \mathrm{M}$ $\mathrm{HNO}_{3}$. Separated ${ }^{225} \mathrm{Ra}$ was reprocessed as needed to provide additional ${ }^{225} \mathrm{Ac}$. 
Actinium separation was performed by Dr. Rose Boll and Karen Murphy at Oak Ridge National Laboratory.

Samples of ${ }^{225} \mathrm{Ac}$ in $1.2 \mathrm{M} \mathrm{HNO}_{3}$ were evaporated to dryness on a hot plate with a continuous flow of air in a V-bottom vial. The dried salt was redispersed in $1 \mathrm{M} \mathrm{HCl}$ and evaporated to dryness under the same conditions. Finally, the sample was redispersed in $0.1 \mathrm{M} \mathrm{HCl}$ and transferred to a $1 \mathrm{~mL}$-bottom vial for synthesis.

\subsubsection{Synthesis of $\left\{\mathrm{La}_{\mathrm{x}} \mathrm{Gd}_{1-\mathrm{x}}\right\} \mathrm{PO}_{4}$ nanoparticle cores}

Lanthanide phosphate nanoparticles were made by modifying a methodology developed by Buissette et al. ${ }^{93}$ Briefly, aqueous solutions of $0.1 \mathrm{M} \mathrm{LaCl}_{3} \cdot 7 \mathrm{H}_{2} \mathrm{O}$ and $\mathrm{GdCl}_{3} \cdot 6 \mathrm{H}_{2} \mathrm{O}$ were mixed in a $1 \mathrm{~mL}$ V-bottom vial with magnetic spin vane. The amount of $\mathrm{La}$ and $\mathrm{Gd}$ added was varied to give $\left\{\mathrm{La}_{\mathrm{x}} \mathrm{Gd}_{1-\mathrm{x}}\right\} \mathrm{PO}_{4}$ nanoparticles where $\mathrm{x}=0,0.25,0.5$, and 1 with a total addition volume of $100 \mu \mathrm{L}$. For biological preparations, $\sim 190 \mathrm{MBq}$ of ${ }^{225} \mathrm{AcCl}_{3}$ in $50 \mu \mathrm{L} 0.1 \mathrm{M} \mathrm{HCl}$ was added to the lanthanide mixture. Preparations for in vitro retention measurements and yield studies used 7.4-9.3 $\mathrm{MBq}$ of ${ }^{225} \mathrm{Ac}$. Preparations for characterization purposes were synthesized without ${ }^{225} \mathrm{Ac}$ addition.

Next, $200 \mu \mathrm{L}$ of $0.1 \mathrm{M}$ sodium tripolyphosphate (TPP) was added to give a clear, colorless solution with a Ln:Na-TPP molar ratio of 1:2. If the solution remained turbid after addition of Na-TPP, it was aspirated with small (10 $\mu \mathrm{L})$ additions of Na-TPP until the solution appeared clear. The resulting solution was then capped and heated at $90^{\circ} \mathrm{C}$ for 3 hours in a Thermo-Scientific heat bath with a custom made aluminum heat block. 
The resulting turbid, white solution of nanoparticles was transferred into a $10 \mathrm{kDa}$ dialysis tube and purified against $18 \mathrm{M} \Omega$ water overnight. The nanoparticles were removed via pipette, producing $2.4 \mathrm{mg}$ of monodisperse particles of $\sim 4 \mathrm{~nm}$ diameter which were characterized by TEM, NAA, and XRD.

\subsubsection{Layering of $\left\{\mathrm{La}_{\mathrm{x}} \mathrm{Gd}_{1-\mathrm{x}}\right\} \mathrm{PO}_{4}$ nanoparticles}

Layers of $\mathrm{LnPO}_{4}$ were added to the crystalline nanoparticle cores by a process of oriented attachment. Nanoparticle cores were collected in a $1.5 \mathrm{~mL}$ microcentrifuge vial centrifuged at 3,000 $\mathrm{g}$ for 3 minutes. The supernatant was decanted via pipette, and nanoparticles were redispersed into a solution containing $400 \mu \mathrm{L} 0.05 \mathrm{M} \mathrm{Na-TPP}$ with $0.05 \mathrm{M} \mathrm{LaCl}_{3}$ and/or $0.05 \mathrm{M} \mathrm{GdCl}_{3}$. Lanthanides were added to give layer composition of $\left\{\mathrm{La}_{\mathrm{x}} \mathrm{Gd}_{1-\mathrm{x}}\right\} \mathrm{PO}_{4}$ where $\mathrm{x}=1,0.5$, or 0 in a total volume of $200 \mu \mathrm{L}$. This nanoparticle suspension was then sonicated for 10 minutes in a bath sonicator, vortexed, and heated for an additional 3 hours in a capped $1 \mathrm{~mL}$ V-bottom vial at $90^{\circ} \mathrm{C}$ to add a layer of $\mathrm{LnPO}_{4}$. The layering procedure was repeated to deposit two or four layers of $\mathrm{LnPO}_{4}$ on top of the core nanoparticle. The final thick, white solution of nanoparticles was then purified by dialysis overnight against $18 \mathrm{M} \Omega$ water. Non-radioactive analogs of the nanoparticles were characterized by EELS-TEM and NAA.

\subsubsection{Gold Shell Addition}

Dialyzed $\mathrm{LnPO}_{4}$ nanoparticles ( 12 mg) were evenly divided between three $5 \mathrm{~mL}$ V-bottom vials ( $4 \mathrm{mg}$ each) with spin vanes. Next, $300 \mu \mathrm{L}$ of $0.1 \mathrm{M}$ sodium citrate was 
added to each vial followed by enough $18 \mathrm{M} \Omega$ water to bring the final volume to $2 \mathrm{~mL}$. The vials were then sonicated for 10 minutes in a bath sonicator and heated to $90^{\circ} \mathrm{C}$. Over the course of 25 minutes, $2.5 \mathrm{~mL}$ of $1 \mathrm{mM} \mathrm{NaAuCl}_{4}(\mathrm{ph}=4)$ was added dropwise, during which time the solution turned the deep purple-red color associated with gold nanoparticles. The solution was heated uncapped for an additional 30-45 minutes and cooled to room temperature. The gold coated nanoparticles were placed next to the NdFeB magnet overnight for separation. The supernatant was decanted via pipette and magnetically active nanoparticles collected for further analysis. Both supernatant and separated particles were counted on a $\mathrm{y}$-spectrometer after establishment of equilibrium to determine separation yield. Figure 3.2.1 shows a purified sample of gold coated nanoparticles following magnetic separation.

In this dissertation, nanoparticle compositions consisting of a core of varying amounts of lanthanide phosphates will be presented with the cation fraction in braces followed by the counteranion. If a radioactive dopant is present, it will be presented in parentheses. For example, a half $\mathrm{LaPO}_{4}$, half $\mathrm{GdPO}_{4}$ nanoparticle core with an ${ }^{225} \mathrm{Ac}$ dopant will be presented as $\left\{\mathrm{La}_{0.5} \mathrm{Gd}_{0.5}\right\}\left({ }^{225} \mathrm{Ac}\right) \mathrm{PO}_{4}$. Additionally, other layers are named using the convention core@shell. Antibodies or linkers attached to the gold surface will be named with a dash followed by the antibody or linker. Thus, a nanoparticle with the aforementioned core, four shells of a lanthanide phosphate, an exterior gold layer, and monoclonal antibody (mAb) 201b antibody would be named $\left\{\mathrm{La}_{0.5} \mathrm{Gd}_{0.5}\right\}\left({ }^{225} \mathrm{Ac}\right) \mathrm{PO}_{4} @ 4$ LnPO ${ }_{4} @ A u-m A b 201 b$. 


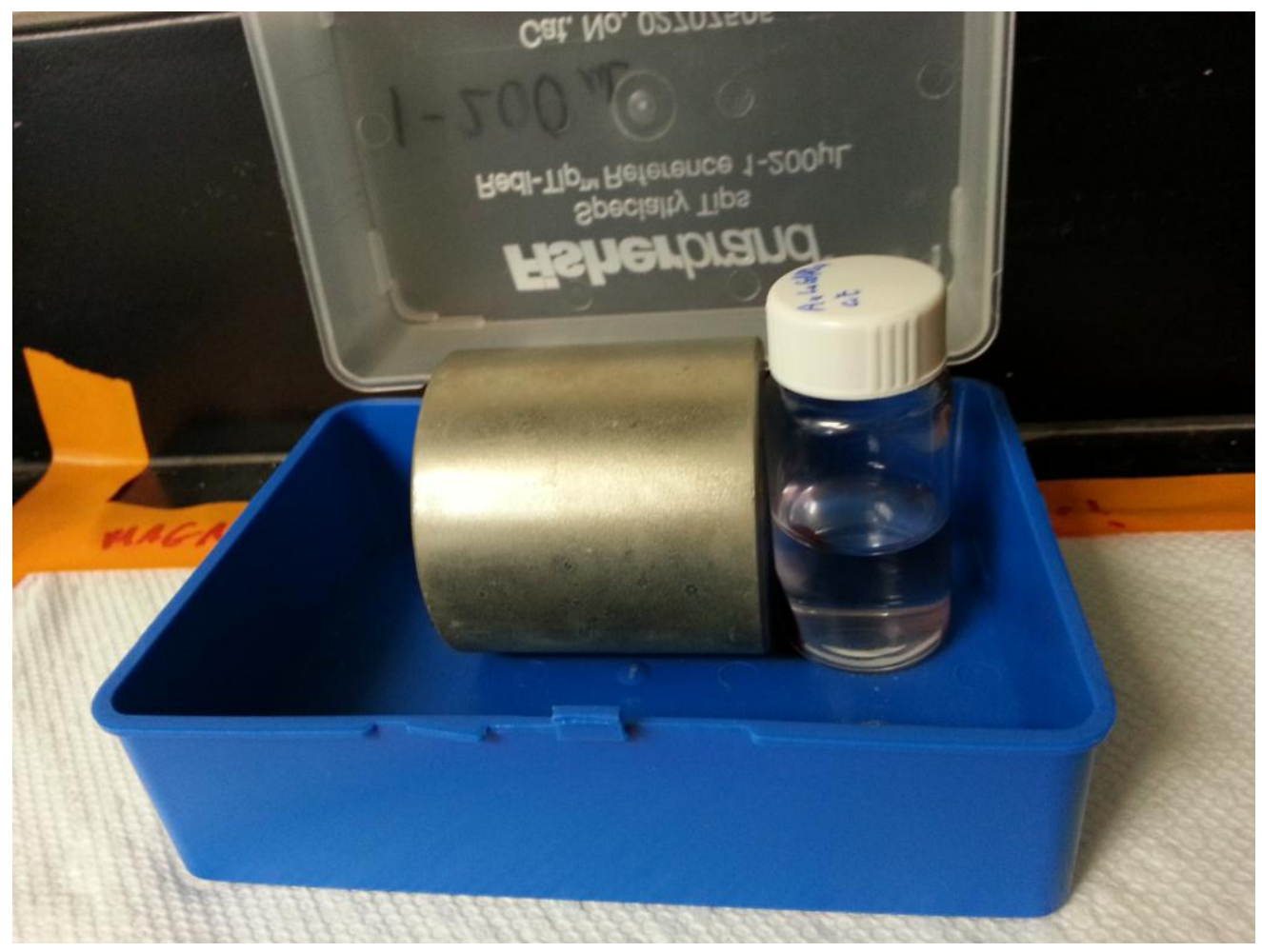

Figure 3.2.1: $\left\{\mathrm{La}_{0.5} \mathrm{Gd}_{0.5}\right\}\left({ }^{225} \mathrm{Ac}\right) \mathrm{PO}_{4} @ 4 \mathrm{GdPO}_{4} @ A u$ nanoparticles after overnight magnetic separation

\subsubsection{Layering Yield Measurements}

La-140 and ${ }^{159} \mathrm{Gd}$ radiotracers were produced at MURR, employing the pneumatic tube irradiation facility. $\mathrm{La}\left(\mathrm{NO}_{3}\right)_{3} \cdot 6 \mathrm{H}_{2} \mathrm{O}(5.8 \mathrm{mg})$ was irradiated for $15 \mathrm{~min}$, generating $28.7 \mathrm{MBq}$ of ${ }^{140} \mathrm{La}$. Similarly, $2.31 \mathrm{MBq}$ of ${ }^{159} \mathrm{Gd}$ was produced by irradiating $6.2 \mathrm{mg}$ of $\mathrm{Gd}\left(\mathrm{NO}_{3}\right)_{3} \cdot 6 \mathrm{H}_{2} \mathrm{O}$ for 45 minutes. $\left\{\mathrm{La}_{0.25} \mathrm{Gd}_{0.75}\right\} \mathrm{PO}_{4}$ nanoparticles in solution as synthesized in 3.2.3 were centrifuged and the supernatant removed. They were redispersed in $400 \mu \mathrm{L}$ of $0.05 \mathrm{M} \mathrm{HCl}$ containing radiotracer ${ }^{140} \mathrm{La}$ and transferred to 
a $5 \mathrm{~mL}$ V-bottom vial. Na-TPP $(800 \mu \mathrm{L}, 0.05 \mathrm{M})$ was added and the solution heated in a capped vial for 3 hours at $90^{\circ} \mathrm{C}$, the layered nanoparticles were moved to a liquid scintillation vial and placed by a NdFeB magnet for separation overnight. Following separation, the supernatant fraction opposite the magnet was decanted via pipette and collected. The supernatant and the nanoparticle fractions were counted on a $\gamma$ spectrometer using identical geometries to determine the fraction of ${ }^{140} \mathrm{La}$ in each component. Nanoparticle composition of gold-coated analogs was measured via NAA at MURR to confirm layering yield. Briefly, three samples of $10 \mu \mathrm{L}$ of nanoparticles were dried down in a $2 / 5$ dram polyethylene vial. Vials were heat sealed, then irradiated for 15 seconds, and allowed to cool for 1 minute. The composition of the $\left\{\mathrm{Gd}_{0.75} \mathrm{La}_{0.25}\right\} \mathrm{PO}_{4} @ 4 \mathrm{LaPO}_{4}$ shells@Au layered nanoparticles was determined by standard comparator NAA using ${ }^{140} \mathrm{La}(10 \mu \mathrm{g}),{ }^{159} \mathrm{Gd}(10 \mu \mathrm{g})$, and ${ }^{198} \mathrm{Au}(1 \mu \mathrm{g})$. Standards were irradiated with the samples in the pneumatic tube system at MURR (neutron flux = of $8 * 10^{13} \mathrm{n} / \mathrm{cm}^{2} / \mathrm{s}$ ) for 15 seconds. Each sample was allowed to decay for 60 seconds, then counted for 30 seconds on a high purity germanium detector.

Layering efficiency of $\mathrm{GdPO}_{4}$ was determined similarly using ${ }^{159} \mathrm{Gd}$ radiotracer. Because the magnetic properties of Gd preclude magnetic separation of layered and non-layered $\mathrm{GdPO}_{4}$ nanoparticles, the layered nanoparticles were allowed to precipitate. The supernatant was decanted via pipette and the activity of the nanoparticles and supernatant were measured on a $y$ spectrometer. Gd yield was 
confirmed by measuring the ratio of $G d: L a$ in the $\left\{\mathrm{Gd}_{0.5} \mathrm{La}_{0.5}\right\} \mathrm{PO}_{4} @ 4 \mathrm{GdPO}_{4}$ shell@Au via NAA and comparing to the theoretical ratio.

\subsubsection{In Vitro Stability Studies}

$\left\{\mathrm{La}_{0.5} \mathrm{Gd}_{0.5}\right\} \mathrm{PO}_{4} @ 4 \mathrm{GdPO}_{4} @$ Au nanoparticles with PEG surface functionalization (3.4.2) were monitored for their stability against aggregation in vitro using UV-Vis spectroscopy. Samples $(0.5 \mathrm{mg})$ were kept in $1 \mathrm{~mL}$ of either DI water, PBS, or BSA for one month. The absorption was measured from $350-700 \mathrm{~nm}$ three times a week to determine stability of both the wavelength and intensity of the surface plasmon resonance band.

\subsubsection{In Vitro Retention Testing}

Retention of the ${ }^{225} \mathrm{Ac}$ decay products was quantified in vitro via dialysis. The ${ }^{225} \mathrm{Ac}$ nanoparticles (14 mg) were placed in a dialysis tube and suspended in $400 \mathrm{~mL}$ of $18 \mathrm{M} \Omega$ water. The dialysis tube was allowed to equilibrate for at least 3 hours (four half-lives of the longest lived $\alpha$-emitting daughter, ${ }^{213} \mathrm{Bi}$ ), before the initial measurement and between each subsequent measurement. A $5 \mathrm{~mL}$ aliquot of the dialysate was taken for radioactivity measurement each day over the course of 20 days (two ${ }^{225} \mathrm{Ac} \mathrm{half-lives).}$ Each dialysate sample was counted immediately to determine ${ }^{221} \mathrm{Fr}$ leakage via the ${ }^{213} \mathrm{Bi}$ activity and then re-analyzed the following day to determine the level of ${ }^{225} \mathrm{Ac}$ in the aliquot using the ${ }^{221} \mathrm{Fr}$ peak. Counts were corrected for decay during sample transport, decay during the counting period, and the decreasing dialysate volume throughout the 
monitoring period. The corrected activity was compared to the level of ${ }^{225}$ Ac activity at the time of separation to determine the ${ }^{221} \mathrm{Fr}$ and ${ }^{225} \mathrm{Ac}$ retention. Both the $218 \mathrm{keV} \gamma$ ray from ${ }^{221} \mathrm{Fr}$ and the $440 \mathrm{keV} \gamma$ ray from ${ }^{213} \mathrm{Bi}$ were used to determine ${ }^{221} \mathrm{Fr}$ retention. Because ${ }^{213} \mathrm{Bi}$ does not move across the dialysis membrane, ${ }^{48}$ all of the ${ }^{213} \mathrm{Bi}$ in the dialysate originates from decay of ${ }^{221} \mathrm{Fr}$ present in the dialysate.

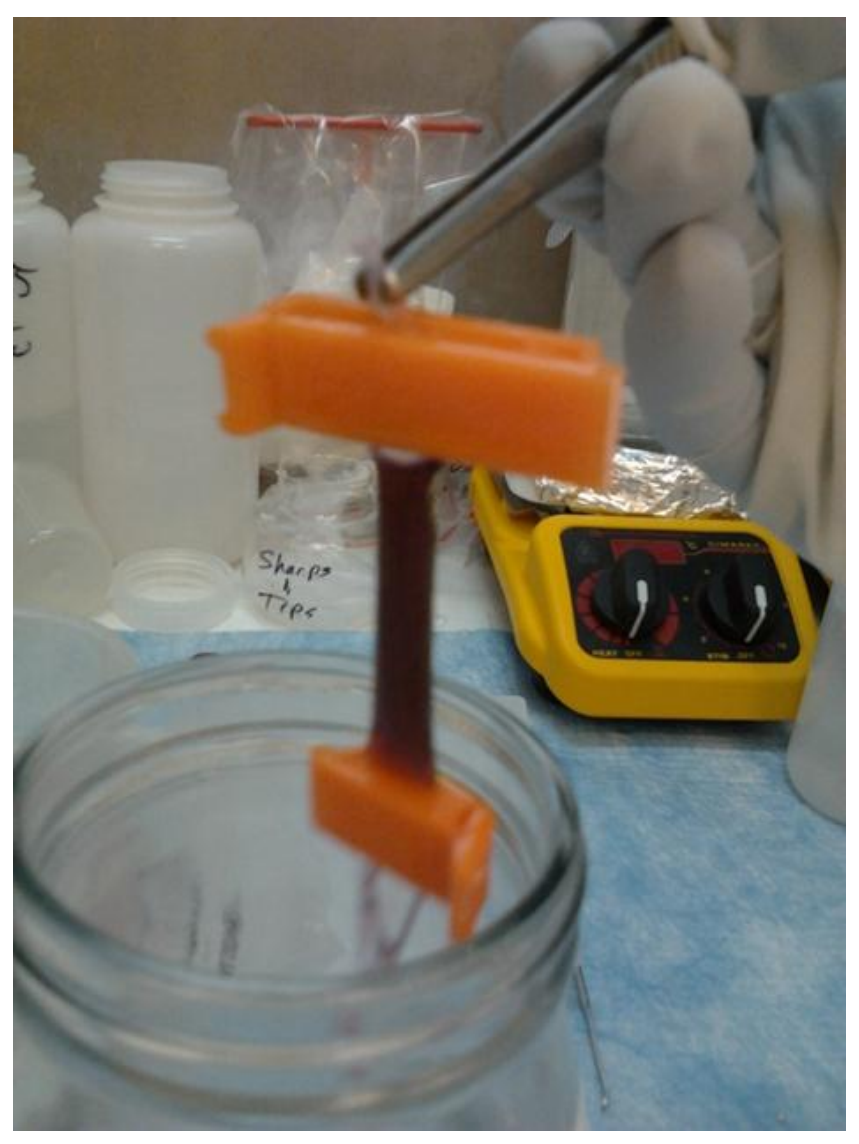

Figure 3.2.2: $\left\{\mathrm{La}_{0.5} \mathrm{Gd}_{0.5}\right\}\left({ }^{225} \mathrm{Ac}\right) \mathrm{PO}_{4} @ 4 \mathrm{GdPO}_{4} @ A u$ nanoparticles loaded into 10 kDa MWCO membrane for in vitro daughter retention studies 


\subsubsection{Layer Leaching Studies}

Nanoparticles cores were synthesized as in 3.2.3. One shell of either ${ }^{159} \mathrm{GdPO}_{4}$ or

${ }^{140} \mathrm{LaPO}_{4}$ was added as in 3.2.4 using radiotracers produced as described in 3.2.6. The nanoparticles were placed in a dialysis membrane and dialyzed for three hours against 1 $\mathrm{L}$ of $18 \mathrm{M} \Omega$ water. The dialysate was then removed and replaced with a new $1 \mathrm{~L}$ of water. Nanoparticles were dialyzed overnight, then counted on a $y$ spectrometer. Twenty $\mathrm{mL}$ of the dialysate was also collected and counted. The dialysate count was corrected for dilution and counting time then compared to the particle activity.

\subsubsection{XRD Studies}

Nanoparticles for XRD studies were prepared as in 3.2.3 with compositions of $\mathrm{LaPO}_{4},\left\{\mathrm{La}_{0.25} \mathrm{Gd}_{0.75}\right\} \mathrm{PO}_{4},\left\{\mathrm{La}_{0.5} \mathrm{Gd}_{0.5}\right\} \mathrm{PO}_{4}$, and $\mathrm{GdPO}_{4}$. Samples were dialyzed and collected in a liquid scintillation vial and dried over low heat $\left(50^{\circ} \mathrm{C}\right)$. The mass of the resulting solid was measured, then ground into a fine powder with a mortar and pestle. The powder was mixed with silicon standard 640c from the National Institute of Standards and Technology and ethanol. Five drops of this mixture was deposited on a zero diffraction silicon plate and allowed to evaporate. Each sample was scanned for one hour from $2 \theta$ values of 15 to 60 degrees. Detailed scan parameters are found in Appendix 2. 


\subsubsection{MRI Relaxivity Measurements}

MRI relaxivity measurements were performed by Dr. Lixin Ma. Nanoparticles were centrifuged and redispersed in $18 \mathrm{M} \Omega$ water at a concentration of $1 \mathrm{mg} / \mathrm{mL}$. This concentration corresponds to a Gd concentration of $0.424 \mathrm{mg} / \mathrm{mL}(2.70 \mathrm{mM})$. Relaxivity values were measured in a field strength of $7 \mathrm{~T}$ at room temperature. Calculations were done assuming these concentrations and mean particle diameter of $26 \mathrm{~nm}$ (determined by TEM).

\subsection{Nanoparticle Characterization Results and Discussion}

$\left\{\mathrm{La}_{x} \mathrm{Gd}_{1-\mathrm{x}}\right\} \mathrm{PO}_{4}$ nanoparticle cores of four different compositions were successfully synthesized. Each exhibits a size of 3-4 $\mathrm{nm}$ as confirmed by TEM. Figure 3.3 .1 shows a characteristic collection of nanoparticles.

Rietveld refinement of XRD patterns indicated that $\mathrm{LaPO}_{4}$ nanoparticles exhibited the rhabdophane phase consistent with the description of Buissette et al. ${ }^{93}$ However, the compositions $\left\{\mathrm{La}_{0.5} \mathrm{Gd}_{0.5}\right\} \mathrm{PO}_{4},\left\{\mathrm{La}_{0.25} \mathrm{Gd}_{0.75}\right\} \mathrm{PO}_{4}$, and $\mathrm{GdPO}_{4}$ exhibit the anhydrous monazite crystal phase. ${ }^{94}$ Nanoparticles consisting of $\mathrm{LaPO}_{4}$ but synthesized in organic media also exhibit the monazite phase. ${ }^{48}$ XRD measurements indicate nanoparticle grain sizes of $4.04 \mathrm{~nm}$ for $\mathrm{LaPO}_{4}, 2.79 \mathrm{~nm}$ for $\left\{\mathrm{La}_{0.5} \mathrm{Gd}_{0.5}\right\} \mathrm{PO}_{4}, 2.91 \mathrm{~nm}$ for $\left\{\mathrm{La}_{0.25} \mathrm{Gd}_{0.75}\right\} \mathrm{PO}_{4}$ and $3.11 \mathrm{~nm}$ for $\mathrm{GdPO}_{4}$. These patterns, along with the accompanying Rietveld refinement, are shown in Figures 3.3.2 A-D. Pure $\mathrm{LaPO}_{4}$ and pure $\mathrm{GdPO}_{4}$ 
exhibited larger grain sizes than their mixed lanthanide counterparts. While the $\mathrm{La}^{3+}$ and $\mathrm{Gd}^{3+}$ ions are similar enough to co-crystallize, the co-crystallization process is retarded relative to the single element crystal.

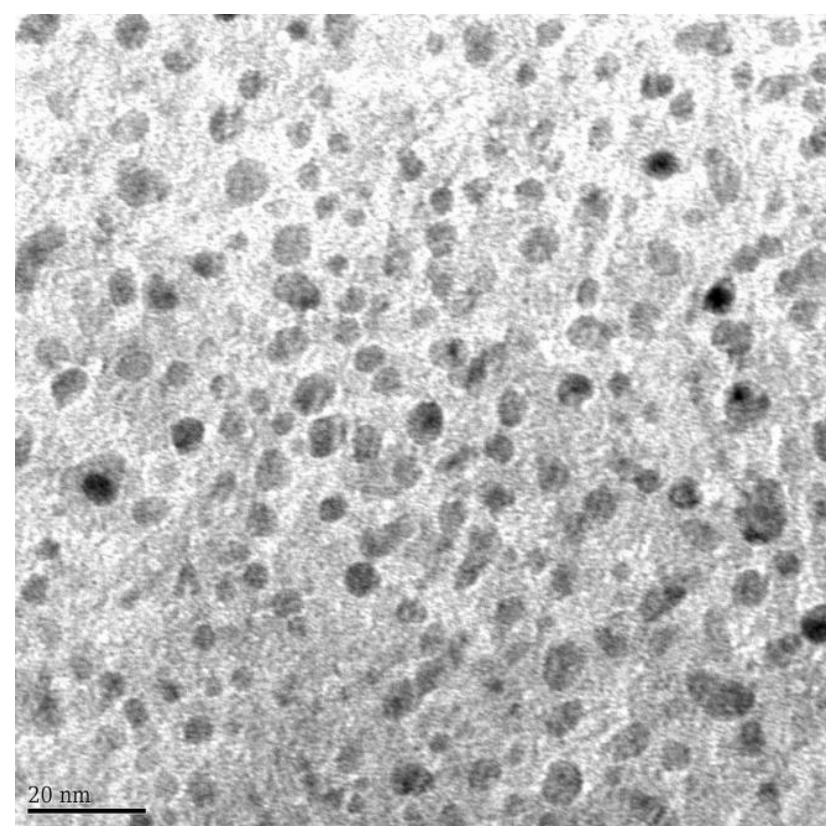

Figure 3.3.1: $\left\{\mathrm{La}_{x} \mathrm{Gd}_{1-\mathrm{x}}\right\} \mathrm{PO}_{4}$ nanoparticle cores exhibit sizes of $\sim 3-4 \mathrm{~nm}$

Size measurements of the nanoparticles using TEM match the XRD grain size values, indicating that the core particles consist of a single crystalline phase. NAA of $\left\{\mathrm{La}_{0.5} \mathrm{Gd}_{0.5}\right\} \mathrm{PO}_{4}$ core nanoparticles after magnetic separation indicates a La to $\mathrm{Gd}$ molar ratio of $1.11 \pm 0.03$. NAA indicates the same ratio in the $\left\{\mathrm{La}_{0.25} \mathrm{Gd}_{0.75}\right\} \mathrm{PO}_{4}$ system is 0.33 \pm 0.02 .

Layering $\left\{\mathrm{La}_{0.5} \mathrm{Gd}_{0.5}\right\} \mathrm{PO}_{4}$ with $\mathrm{GdPO}_{4}$ occurs with a chemical yield of $99 \%$. This yield, combined with a core Gd:La ratio of 0.90 , results in a theoretical Gd:La for the 
$\left\{\mathrm{La}_{0.5} \mathrm{Gd}_{0.5}\right\} \mathrm{PO}_{4} @ 4 \mathrm{GdPO}_{4}$ shell nanoparticle of 7.91. The experimental ratio as

measured by NAA is $7.5 \pm 0.9$. Nanoparticle growth after each shell addition was

monitored by TEM and is shown in table 3.3.1. These $\left\{\mathrm{La}_{0.5} \mathrm{Gd}_{0.5}\right\} \mathrm{PO}_{4} @ 4 \mathrm{GdPO}_{4}$ shell nanoparticles have a mean diameter of $22 \pm 8 \mathrm{~nm}$. After one day of leaching in $18 \mathrm{M} \Omega$ water, $99.96 \pm 0.06$ of the tracer activity in the Gd shell remained attached to the core nanoparticle.
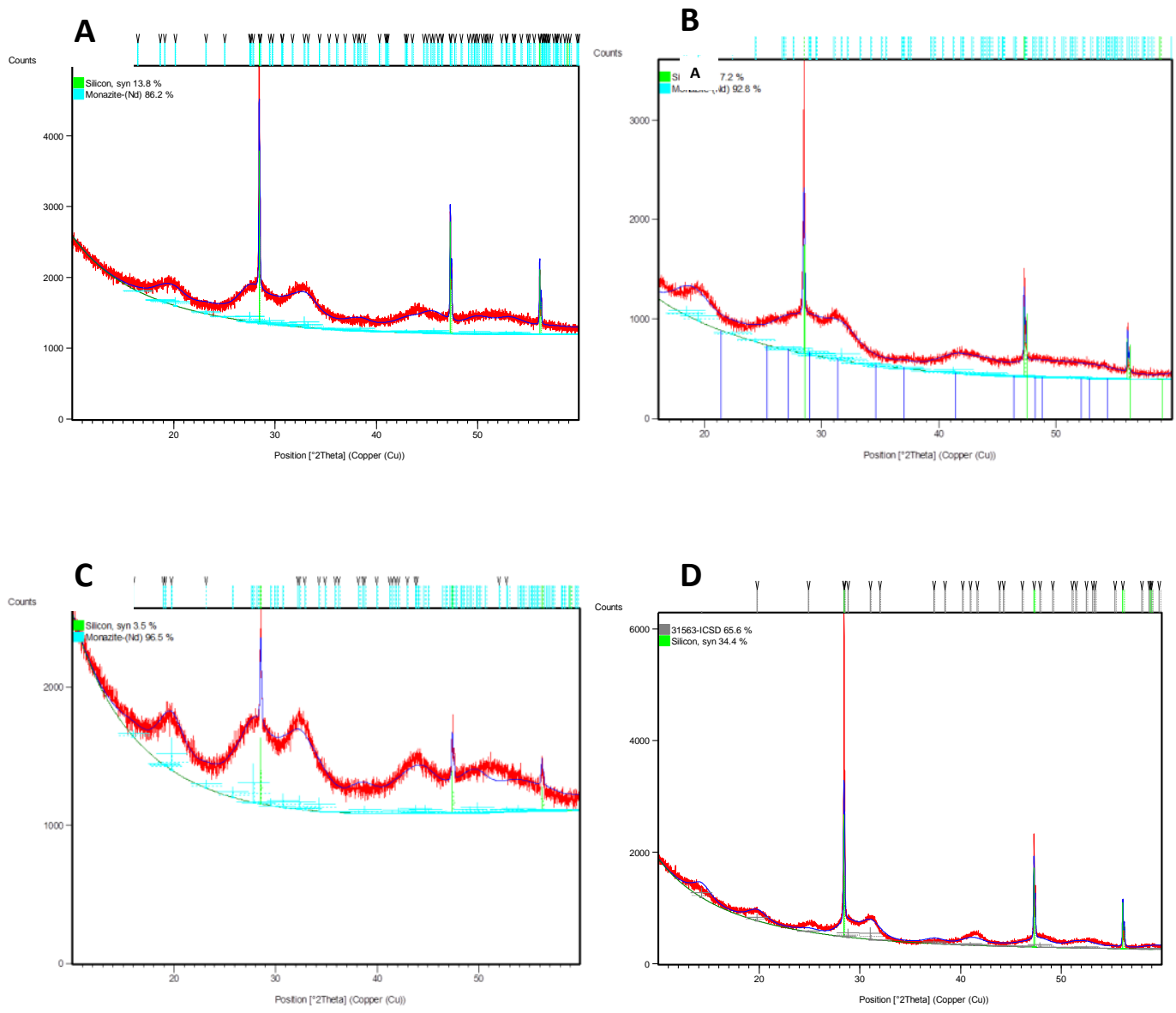

Figure 3.3.2 A-D: XRD patterns and Rietveld refinement for $\mathrm{GdPO}_{4}(\mathrm{~A}),\left\{\mathrm{La}_{0.5} \mathrm{Gd}_{0.5}\right\} \mathrm{PO}_{4}$ (B), $\mathrm{LaPO}_{4}(\mathrm{C})$, and $\left\{\mathrm{La}_{0.25} \mathrm{Gd}_{0.75}\right\} \mathrm{PO}_{4}$ (D). Red lines indicate the calculated spectra while the blue line represents the experimentally measured data. 
Table 3.3.1: Nanoparticle growth of $\left\{\mathrm{La}_{0.5} \mathrm{Gd}_{0.5}\right\} \mathrm{PO}_{4} @ 4 \mathrm{GdPO}_{4}$ shell@Au

\begin{tabular}{|l|c|}
\hline \multicolumn{1}{|c|}{ Particle System } & $\begin{array}{c}\text { Diameter } \\
(\mathrm{nm})\end{array}$ \\
\hline$\left\{\mathrm{La}_{0.5} \mathrm{Gd}_{0.5}\right\} \mathrm{PO}_{4}$ Core & $5.0 \pm 1.5$ \\
\hline$\left\{\mathrm{La}_{0.5} \mathrm{Gd}_{0.5}\right\} \mathrm{PO}_{4} @ 1$ shell $\mathrm{GdPO}_{4}$ & $7.8 \pm 2.8$ \\
\hline$\left\{\mathrm{La}_{0.5} \mathrm{Gd}_{0.5}\right\} \mathrm{PO}_{4} @ 2$ shells $\mathrm{GdPO}_{4}$ & $9.9 \pm 2.6$ \\
\hline$\left\{\mathrm{La}_{0.5} \mathrm{Gd}_{0.5}\right\} \mathrm{PO}_{4} @ 3$ shells $\mathrm{GdPO}_{4}$ & $13.3 \pm 1.8$ \\
\hline$\left\{\mathrm{La}_{0.5} \mathrm{Gd}_{0.5}\right\} \mathrm{PO}_{4} @ 4$ shells $\mathrm{GdPO}_{4}$ & $22.4 \pm 7.7$ \\
\hline$\left\{\mathrm{La}_{0.5} \mathrm{Gd}_{0.5}\right\} \mathrm{PO}_{4} @ 4$ shells $\mathrm{GdPO}_{4} @ \mathrm{Au}$ & $26.8 \pm 4.9$ \\
\hline
\end{tabular}

Radiotracer experiments indicate that $\mathrm{LaPO}_{4}$ shells add with a $91 \%$ chemical yield. Utilizing this layering yield and a core composition with La:Gd ratio of 0.33 , the expected La:Gd ratio of a $\left\{\mathrm{La}_{0.25} \mathrm{Gd}_{0.75}\right\} \mathrm{PO}_{4} @ 4 \mathrm{LaPO}_{4}$ nanoparticle is 5.17. Experimental measurements with NAA indicate that the actual nanoparticle has a La:Gd ratio of $5.1 \pm$ 0.7. TEM indicates that the $\left\{\mathrm{La}_{0.25} \mathrm{Gd}_{0.75}\right\} \mathrm{PO}_{4} @ 4 \mathrm{LaPO}_{4}$ nanoparticle has a mean diameter of $19.9 \pm 6.5 \mathrm{~nm}$. Leaching studies in $18 \mathrm{M} \Omega$ water indicate that $99.94 \pm 0.13$ $\%$ of the La tracer in the shell remains attached after one day. Overnight magnetic separation occurs with a $89 \%$ yield.

Each shell addition process utilizes identical amounts of Ln and TPP. An epitaxial growth mechanism with this reaction scheme would generate successively thinner shells of equal volume. Thus, each successive shell would generate a smaller change in 
diameter of the nanoparticle. However, this phenomenon is not observed (Table 3.3.1). Given these observations, the nanoparticles likely grow through a process of oriented alignment and attachment as recently described by Li et al. ${ }^{95}$ In this process, nanoparticle growth occurs when smaller nanocrystals combine in a controlled fashion. Dilute starting material, which is insufficient to create new nanoparticles in solution, will fill in around the attachment point to maintain spherical nanoparticles. The added lanthanide ions and the orthophosphate produced by thermolysis of Na-TPP thus incorporate into the crystal structure. While the $\mathrm{GdPO}_{4}$ and $\mathrm{LaPO}_{4}$ layers possess slightly different crystal systems, they both generate near quantitative layer deposition onto the mixed $\left\{\mathrm{La}_{0.5} \mathrm{Gd}_{0.5}\right\} \mathrm{PO}_{4}$ or $\left\{\mathrm{La}_{0.25} \mathrm{Gd}_{0.75}\right\} \mathrm{PO}_{4}$ cores.

Gold shells add easily to all compositions of the nanoparticles. EELS-TEM of gold gives a clear picture of the size of the added shell. A characteristic image is shown below in Figure 3.3.3. Ratios of Au:Ln in the particles based off of a $0.5 \mathrm{~nm}$ gold shell on top of a $22.4 \mathrm{~nm} \mathrm{LnPO}_{4}$ nanoparticle (Figure 3.3.3) predict a Au:Ln ratio of 0.65. NAA measures a ratio in the particles of $0.61 \pm 0.01$. Nanoparticles consisting of $\left\{\mathrm{La}_{0.5} \mathrm{Gd}_{0.5}\right\} \mathrm{PO}_{4} @ 4 \mathrm{GdPO}_{4}$ shell@Au-PEG remain stable against aggregation in all biological mimic solutions tested (water, PBS, BSA) over the course of a month.

Addition of a gold shell onto the surface also raises the possibility of simultaneously creating pure gold nanoparticles. Pure gold nanoparticles would possess identical surface chemistry (including properties such as plasmon resonance, Figure 3.3.4) and similar size/mass to the layered construct, making them difficult to separate. 
The gadolinium in the layered structure allows for magnetic separation of the layered construct from any pure gold nanoparticles formed in the reaction. Without separation, pure gold nanoparticles would effectively lower the specific activity of the construct.

Targeting agents on the pure gold nanoparticles would compete with therapeutic layered nanoparticles for the limited number of receptor sites in vivo, lowering the targeting efficacy of the layered nanoparticles and decreasing the delivered therapeutic dose.

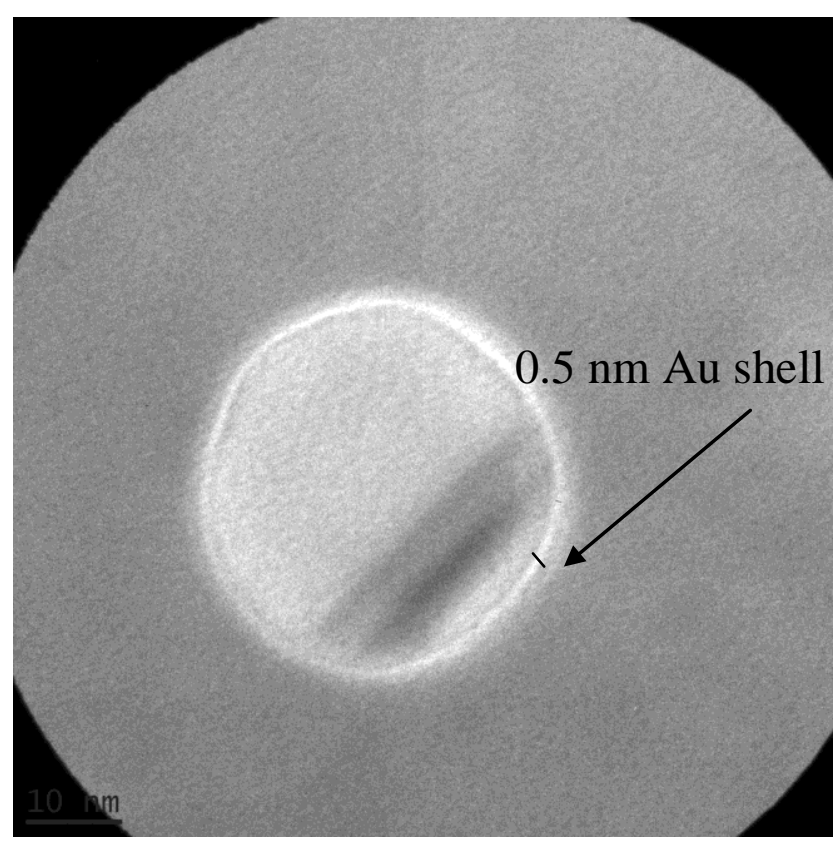

Figure 3.3.3: EELS-TEM of a characteristic $\left\{\mathrm{La}_{0.5} \mathrm{Gd}_{0.5}\right\} \mathrm{PO}_{4} @ 4 \mathrm{GdPO}_{4}$ shell@Au nanoparticle showing the width of the gold surface layer

While lanthanum and gadolinium are both present in the nanoparticle solution, this does not confirm that they are co-crystallized. There could be separate $\mathrm{LaPO}_{4}$ and $\mathrm{GdPO}_{4}$ nanoparticles without mixture of lanthanides. This possibility was discounted by 
two experiments. First, NAA confirms that magnetically separating the nanoparticles does not enrich the nanoparticles in Gd content. Nanoparticles that contain only $\mathrm{LaPO}_{4}$ will not be attracted to the magnet. However, La:Gd ratios remain constant after separation. Next, EELS-TEM confirms the presence of all three elements, lanthanum, gadolinium, and gold, in a cluster of particles. Those images are shown in Figure 3.3.5.

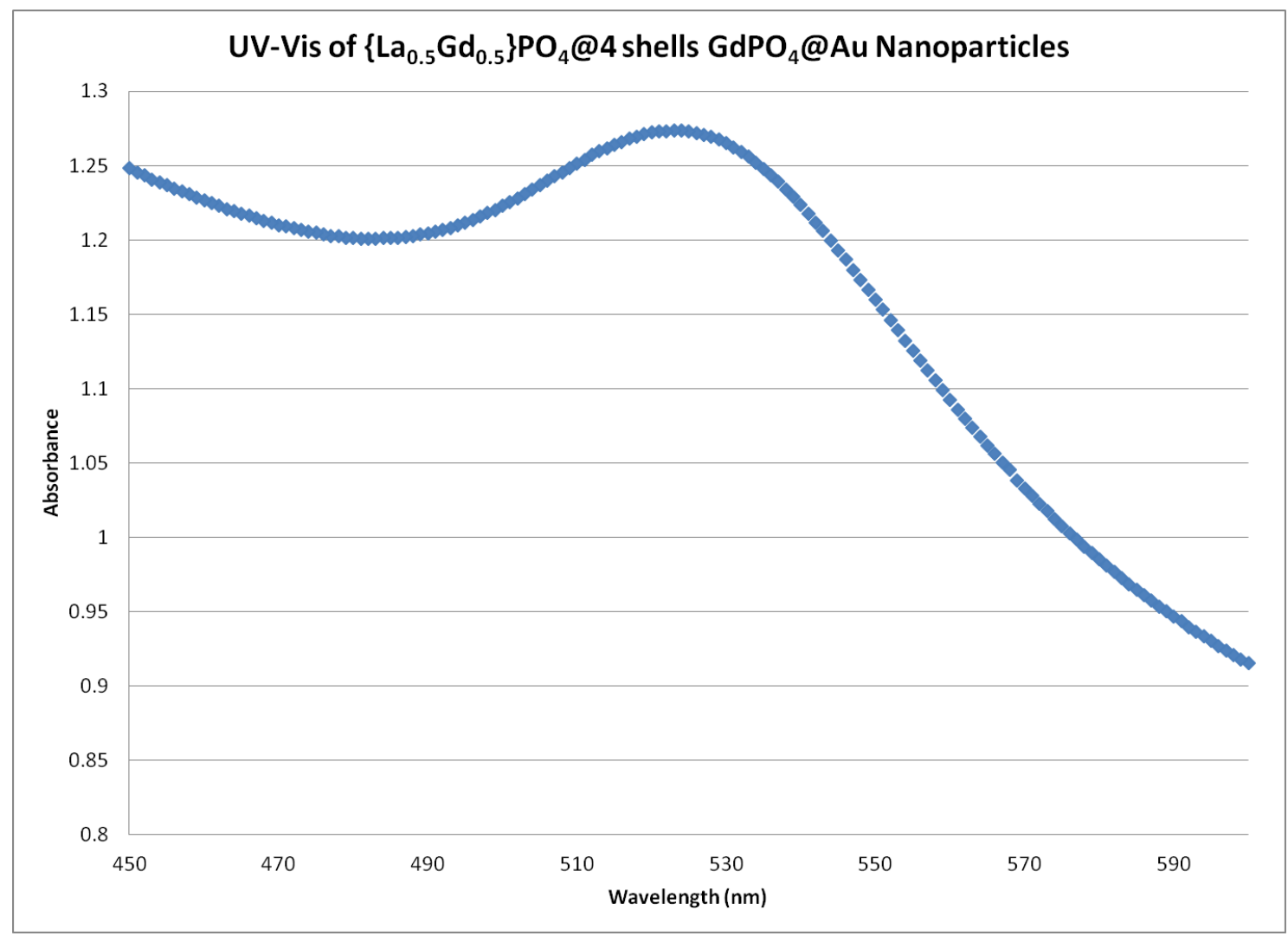

Figure 3.3.4: UV-Vis of $\left\{\mathrm{La}_{0.5} \mathrm{Gd}_{0.5}\right\} \mathrm{PO}_{4} @ 4 \mathrm{GdPO}_{4}$ shell@Au nanoparticles. Absorbance peak occurs at $523 \mathrm{~nm}$ 


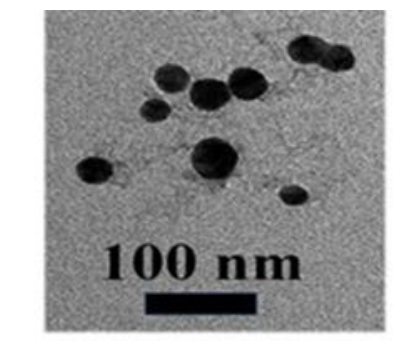

$\mathrm{Au}\left\{\mathrm{La}_{0.5} \mathrm{Gd}_{0.5} \mathrm{POO}_{4}\right.$

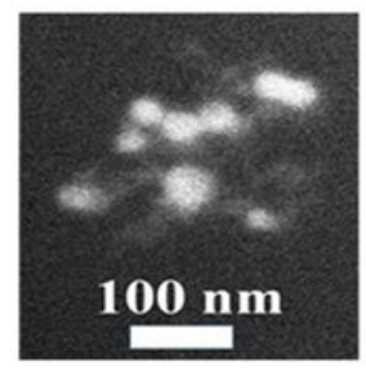

Gd EELS

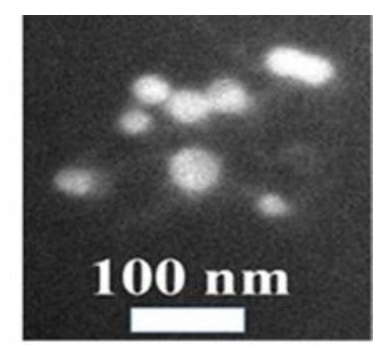

La EELS

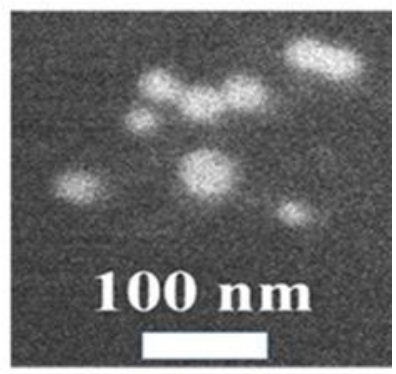

Au EELS

Figure 3.3.5: EELS-TEM confirms the presence of lanthanum, gadolinium, and gold in each nanoparticle. The top left image shows the standard TEM of the same particles.

The synthesis of the gold coated, four $\mathrm{LnPO}_{4}$ shell system has an overall radiochemical yield of $57.5 \%$. Correcting this value for the decay of ${ }^{225} \mathrm{Ac}$, the overall chemical yield for incorporation of ${ }^{225} \mathrm{Ac}$ in the nanoparticles is $\sim 76 \%$ at the end of the 4 day synthesis. Figure 3.3.6 shows a schematic of a completed $\left\{\operatorname{La}_{x} \mathrm{Gd}_{1-\mathrm{x}}\right\} P \mathrm{PO}_{4} @ 4$ LnPO ${ }_{4} @ A u$ nanoparticle.

$\mathrm{GdPO}_{4}$ nanoparticles are currently being investigated as potential MRI contrast

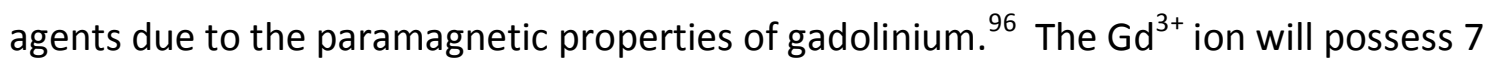
unpaired electrons, the highest number possible using commonly accessible orbitals. $\mathrm{MRI}$ measurements of the $\mathrm{GdPO}_{4}$ shell system indicate $r_{1}$ values of $1.00 \pm 0.01 \mathrm{~s}^{-1} \mathrm{mM}^{-1}$ 
and $r_{2}$ values of $44.0 \pm 4.2 \mathrm{~s}^{-1} \mathrm{mM}^{-1}$. The $r_{2}$ value of these nanoparticles falls on the lower end of approved contrast agents. MRI contrast requires interaction between the gadolinium and the water medium. We hypothesize that the gold shell in the nanoparticles partially inhibits the action of the large quantity of gadolinium in the nanoparticle. MRI contrast remains a compelling application for the $\mathrm{GdPO}_{4}$ shell nanoparticles when combined with their therapeutic and potential SPECT capabilities. While unlikely to win approval as a standalone contrast agent, the MRI properties of the $\left\{\mathrm{La}_{0.5} \mathrm{Gd}_{0.5}\right\} \mathrm{PO}_{4} @ 4 \mathrm{GdPO}_{4}$ shell@Au system warrant further study. The collection efficiency with overnight magnetic separation of $\left\{\mathrm{La}_{0.5} \mathrm{Gd}_{0.5}\right\} \mathrm{PO}_{4} @ 4 \mathrm{GdPO}_{4}$ shell@Au nanoparticles is typically on the order of $\sim 90 \%$.

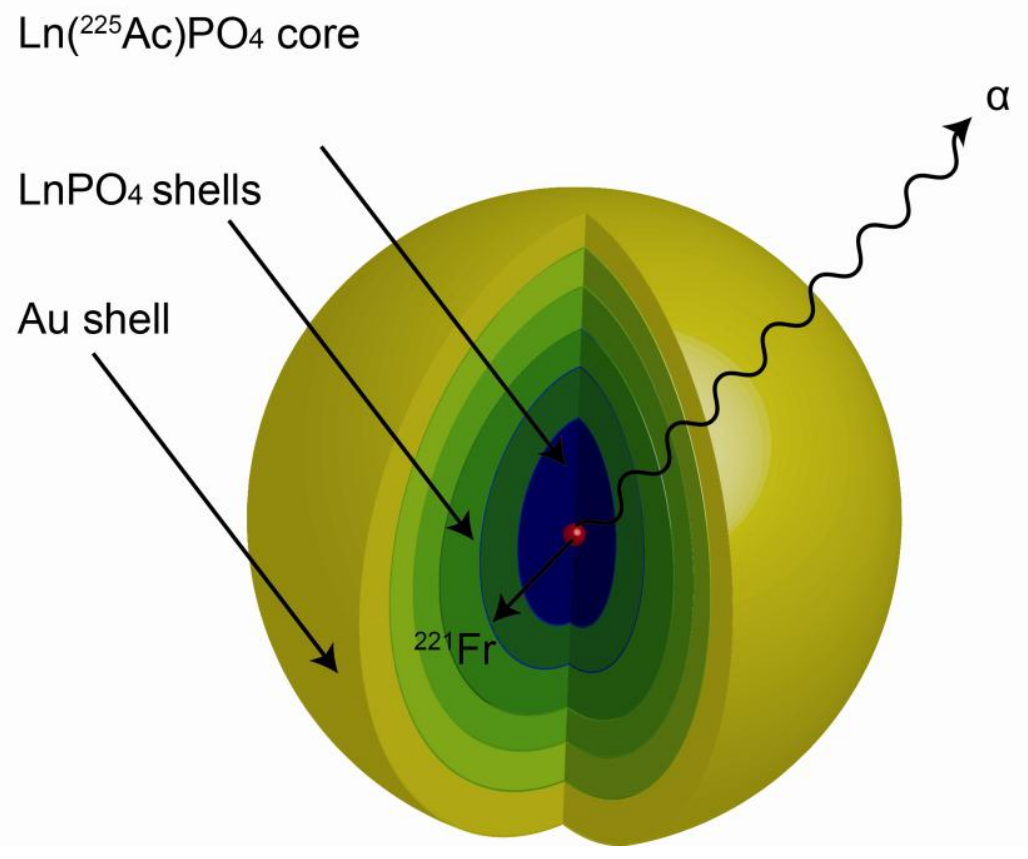

Figure 3.3.6: Idealized schematic of a $\left\{\mathrm{La}_{x} \mathrm{Gd}_{1-\mathrm{x}}\right\} \mathrm{PO}_{4} @ 4 \mathrm{LnPO}_{4} @ \mathrm{Au}$ nanoparticle. The decay of ${ }^{225} \mathrm{Ac}$ and subsequent retention of the ${ }^{221} \mathrm{Fr}$ daughter product are illustrated. 
Retention of ${ }^{225} \mathrm{Ac}$ in the nanoparticle depends on the core composition and the number/type of shells added. $\left\{\mathrm{La}_{0.5} \mathrm{Gd}_{0.5}\right\} \mathrm{PO}_{4}$ core nanoparticles without additional shells retain $99.04 \% \pm 0.07 \%$ of ${ }^{225} \mathrm{Ac}$ throughout the three week observation period. Adding two shells of $\left\{\mathrm{La}_{0.5} \mathrm{Gd}_{0.5}\right\} \mathrm{PO}_{4}$ to this core increases ${ }^{225} \mathrm{Ac}$ retention to $99.42 \pm$ $0.02 \%$. Systems with four shells retain ${ }^{225} \mathrm{Ac}$ to an even higher degree. The $\left\{\mathrm{La}_{0.25} \mathrm{Gd}_{0.75}\right\} \mathrm{PO}_{4} @ 4 \mathrm{LaPO}_{4}$ shell@Au system sequesters $99.97 \pm 0.01 \%$ of the ${ }^{225} \mathrm{Ac}$ while the $\left\{\mathrm{La}_{0.5} \mathrm{Gd}_{0.5}\right\} \mathrm{PO}_{4} @ 4 \mathrm{GdPO}_{4}$ shells@Au system retains $99.996 \pm 0.002 \%$.

Nanoparticles where La comprises a portion of the core retain a higher proportion of ${ }^{225} \mathrm{Ac}$ than compositions that include only $\mathrm{Gd}$. The increased retention likely results from isomorphism between ${ }^{225} \mathrm{Ac}^{3+}$ and $\mathrm{La}^{3+}$ ions. For 8-coordinate configurations, the ${ }^{225} \mathrm{Ac}^{3+}$ has an ionic radius of $1.36 \AA$ compared with $1.30 \AA$ for $\mathrm{La}^{3+}$ and $1.19 \AA$ for $\mathrm{Gd}^{3+} .97$ Further, ${ }^{225} \mathrm{Ac}$ retention in the $\left\{\mathrm{La}_{0.5} \mathrm{Gd}_{0.5}\right\} \mathrm{PO}_{4} @ 4 \mathrm{GdPO}_{4}$ shell@Au system improves by an order of magnitude over the corresponding $4 \mathrm{LaPO}_{4}$ shell system. This difference results from the larger layering yield observed in the $\mathrm{GdPO}_{4}$ shell addition (larger particles with thicker shells). The ${ }^{225}$ Ac must diffuse through a longer path to escape the $\mathrm{GdPO}_{4}$ shells than the $\mathrm{LaPO}_{4}$ system. Due to the lower overall gadolinium content in the lanthanum shell system, the ratio of $\mathrm{Gd}: \mathrm{La}$ used in the core of the $\mathrm{LaPO}_{4}$ shell particles was increased from 1:1 to $3: 1$ so that the nanoparticles could be magnetically separated following gold shell addition. Though this new $\left\{\mathrm{Gd}_{0.75} \mathrm{La}_{0.25}\right\} \mathrm{PO}_{4}$ core still contains the ${ }^{225} \mathrm{Ac}$ to a high degree, retention was measured 
to be slightly lower than the value obtained with the $\left\{G_{0.5} L_{0.5}\right\} P O_{4}$ core. A summary of the ${ }^{225} \mathrm{Ac}$ and ${ }^{221} \mathrm{Fr}$ retention values are given in Table 3.3.2.

Table 3.3.2: Retention of ${ }^{225} \mathrm{Ac}$ and its daughter ${ }^{221} \mathrm{Fr}$ in different nanoparticle constructs

\begin{tabular}{|l|c|c|c|c|}
\hline Core & Shell Material & \# of Shells & ${ }^{225}$ Ac retention ${ }^{\text {a }}$ & ${ }^{221}$ Fr retention $^{\text {b }}$ \\
\hline$\left\{\mathrm{La}_{0.5} \mathrm{Gd}_{0.5}\right\} \mathrm{PO}_{4}$ & None & 0 & $99.16 \pm 0.02 \%$ & $60.2 \% \pm 3.0 \%$ \\
\hline$\left\{\mathrm{La}_{0.5} \mathrm{Gd}_{0.5}\right\} \mathrm{PO}_{4}$ & $\left\{\mathrm{La}_{0.5} \mathrm{Gd}_{0.5}\right\} \mathrm{PO}_{4}$ & 2 & $99.42 \pm 0.02 \%$ & $69.2 \pm 1.7 \%$ \\
\hline$\left\{\mathrm{La}_{0.25} \mathrm{Gd}_{0.75}\right\} \mathrm{PO}_{4}$ & $\mathrm{LaPO}_{4}$ & 4 & $99.96 \pm 0.01 \%$ & $79.4 \pm 1.6 \%$ \\
\hline$\left\{\mathrm{La}_{0.25} \mathrm{Gd}_{0.75}\right\} \mathrm{PO}_{4}$ & $\mathrm{GdPO}_{4}$ & 4 & $99.98 \pm 0.02 \%$ & $90.9 \pm 0.9 \%$ \\
\hline$\left\{\mathrm{La}_{0.5} \mathrm{Gd}_{0.5}\right\} \mathrm{PO}_{4}$ & LaPO $_{4}$ & 4 & $99.97 \pm 0.01 \%$ & $79.9 \pm 1.9 \%$ \\
\hline$\left\{\mathrm{La}_{0.5} \mathrm{Gd}_{0.5}\right\} \mathrm{PO}_{4}$ & $\mathrm{GdPO}_{4}$ & 4 & $99.996 \pm 0.002 \%$ & $89.3 \pm 1.8 \%$ \\
\hline
\end{tabular}

Measurements of ${ }^{221} \mathrm{Fr}$ retention indicate that the sequestration efficiency also depends on the number and composition of shells added to the nanoparticle. Unlike ${ }^{225} \mathrm{Ac}$, retention of ${ }^{221} \mathrm{Fr}$ also depends to some degree on the time since synthesis. The amount of ${ }^{221} \mathrm{Fr}$ retention typically drops slightly over the course of the first week of observation, then stabilizes during the second and third week. After three weeks, the $\left\{\mathrm{Gd}_{0.5} \mathrm{La}_{0.5}\right\}\left({ }^{225} \mathrm{Ac}\right) \mathrm{PO}_{4}$ core contains $60.2 \% \pm 3.0 \%$ of the ${ }^{221} \mathrm{Fr}$ daughter. Retention increases to $69.2 \pm 1.7 \%$ after adding two shells of $\left\{\mathrm{Gd}_{0.5} \mathrm{La}_{0.5}\right\} \mathrm{PO}_{4}$. Four shells of $\mathrm{LnPO}_{4}$ further increases ${ }^{221} \mathrm{Fr}$ retention values. The $\left\{\mathrm{La}_{0.25} \mathrm{Gd}_{0.75}\right\} \mathrm{PO}_{4} @ 4 \mathrm{LaPO}_{4} @ A u$ system initially retains $89.8 \pm 0.5 \%$ of the ${ }^{221} \mathrm{Fr}$. This retention decreases over the course of the first week and stabilizes at $79.9 \pm 1.9 \%$. The $\left\{\mathrm{La}_{0.5} \mathrm{Gd}_{0.5}\right\} P \mathrm{PO}_{4} @ 4 \mathrm{GdPO} \mathrm{A}_{4} @ A u$ system 
initially retains $97.8 \pm 0.5 \%$ of the ${ }^{221} \mathrm{Fr}$ daughter. This retention value also decreases over the course of a week, stabilizing at $89.3 \pm 1.8 \%$. Francium-221 retention data is presented in Figure 3.3.7. For comparison purposes, previously reported $\mathrm{La}\left({ }^{225} \mathrm{Ac}\right) \mathrm{PO}_{4}$ nanoparticles synthesized in organic solvents retain only $\sim 50 \%$ of the ${ }^{221} \mathrm{Fr}$ daughter. ${ }^{48}$

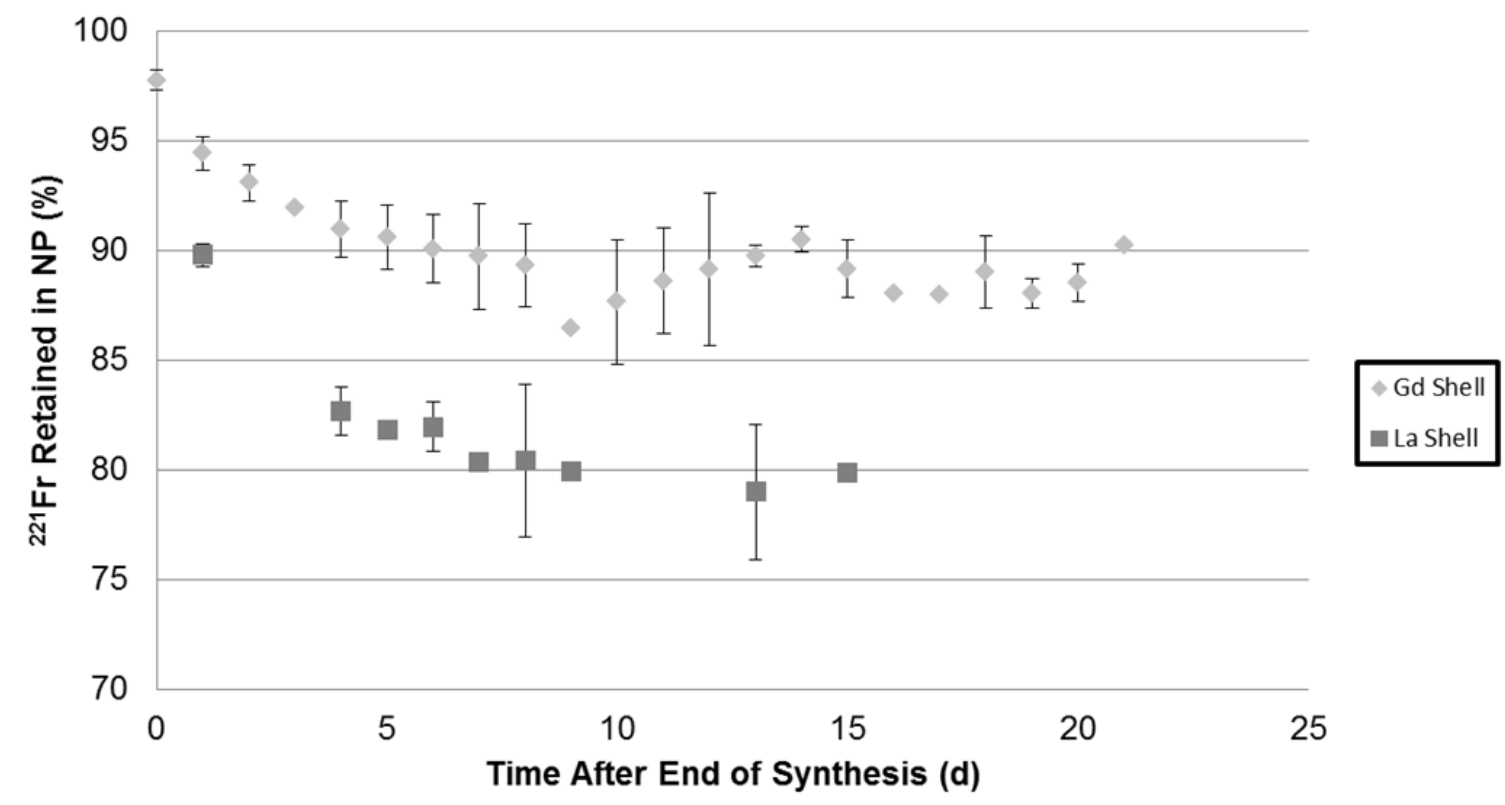

Figure 3.3.7: ${ }^{221} \mathrm{Fr}$ retention data for the $\left\{\mathrm{La}_{0.5} \mathrm{Gd}_{0.5}\right\} \mathrm{PO}_{4} @ 4 \mathrm{GdPO}_{4}$ shell@Au and $\left\{\mathrm{La}_{0.25} \mathrm{Gd}_{0.75}\right\} \mathrm{PO}_{4} @ 4 \mathrm{LaPO}_{4}$ shell@Au nanoparticle systems

Though the layering efficiencies of both the $\mathrm{LaPO}_{4}$ and $\mathrm{GdPO}_{4}$ systems are $>90 \%$, $\mathrm{GdPO}_{4}$ shells clearly contain the ${ }^{221} \mathrm{Fr}$ daughter to a higher degree than their $\mathrm{LaPO}_{4}$ shell counterparts in vitro. Part of this improved retention can be attributed to the higher chemical layering yield of $\mathrm{GdPO}_{4}$. Nanoparticles with $\mathrm{GdPO}_{4}$ shells are slightly larger and have more marginally more material that the recoiling daughter must pass through to escape. Additionally, the higher electron density of the $\mathrm{GdPO}_{4}$ creates a slightly higher 
stopping power. While daughter products are retained in the nanoparticle, the associated $\alpha$-particles lose a negligible fraction of their energy in traversing the nanoparticle. A $6 \mathrm{MeV} \alpha$-particle loses less than $0.2 \%$ of its energy in the layered nanoparticle whereas the range of the $100 \mathrm{keV}$ recoiling daughters is $\sim 20 \mathrm{~nm}$ in bulk $\mathrm{LnPO}_{4}{ }^{50}$ Moreover, a portion of the kinetic energy of the daughter particle may be transferred to the entire particle. If a portion of the recoil energy is distributed throughout the highly structured crystalline lattice, the recoiling range of the daughter radionuclides will be significantly decreased. ${ }^{98}$ To some extent, the nanoparticle may be able to recoil as a unit in solution, absorbing some of the daughter recoil energy and retarding the daughter radionuclide's motion out of the center of the nanoparticle. Again, the more massive $\mathrm{GdPO}_{4}$ shell system would absorb slightly more recoil energy than the lighter $\mathrm{LaPO}_{4}$ shell system.

\subsection{Biological Targeting Experimental}

\subsubsection{Materials and Measurements}

All animal experiments were performed according to either the protocols set forth by the University of Missouri-Columbia Institutional Animal Care and Use Committee approved protocol number 7055 or according to the Institutional Animal Care and Use Committee of the University of Tennessee approved protocol 1502. Chemicals were used as received from Sigma Aldrich unless otherwise noted. Anti- 
synaptophysin and anti-glial fibrillary acidic protein (GFAP) antibodies were received from Dako. MAb 201b and EMT-6 tumor cells were provided by Dr. Stephen Kennel. Lipoamide-dPEG ${ }_{12}-\mathrm{COOH}$ was received from Quanta Biodesign. Sections 3.4.5 - 3.4.8 and 3.4.10 were performed by Dr. Stephen Kennel and Dr. Jonathan Wall at the University of Tennessee - Knoxville Graduate School of Medicine.

\subsubsection{Surface Functionalization}

Nanoparticles (12 mg in $10 \mathrm{~mL}$ water) were functionalized using a water soluble lipoamide- $\mathrm{dPEG}_{12}-\mathrm{COOH}$ linker. The structure of the linker is shown in Figure 3.4.1. Two mg of dPEG were added to a solution of $12 \mathrm{mg}$ of $\left\{\mathrm{La}_{x} \mathrm{Gd}_{1-\mathrm{x}}\right\} \mathrm{PO}_{4} @ 4 \mathrm{LnPO}_{4}$ shell@Au nanoparticles in $5 \mathrm{~mL}$ of PBS, followed by $6 \mathrm{mg}$ of tris(2-carboxyethyl)phosphine (TCEP) reducing agent to cleave the disulfide bond and facilitate citrate displacement. The solution $\mathrm{pH}$ was adjusted to 7 with $0.1 \mathrm{M} \mathrm{NaOH}$ monitored by $\mathrm{pH}$ test strips and the reaction mixture was stirred for 4 hours. Nanoparticles were centrifuged at $3000 \mathrm{~g}$ for 3 minutes and the supernatant containing excess dPEG decanted via pipette. Connection of the linker was confirmed by a shift in the plasmon resonance near $540 \mathrm{~nm}$ as monitored by UV-Vis spectroscopy before and after the addition of the linker. The surface plasmon resonance is highly sensitive to changes in the dielectric constant at the gold surface. A shift in the plasmon resonance indicates a change in the surface environment of the nanoparticle and is indicative of antibody attachment. 


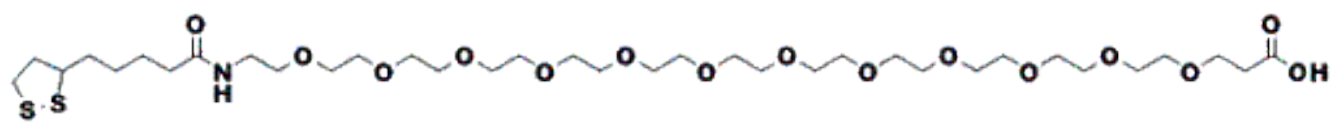

Figure 3.4.1: Structure of lipoamide- $\mathrm{dPEG}_{12}-\mathrm{COOH}$ linker

\subsubsection{Antibody Conjugation}

Five separate antibodies (anti-synaptophysin, anti-GFAP, mAb 201b, HuM-195, and panitumumab) were individually conjugated to the nanoparticles using the same basic procedure. Each antibody is an immunoglobin $\mathrm{G}$ type with a molecular mass of $\sim 150 \mathrm{kDa}$. In a standard conjugation, $0.5 \mathrm{mg}$ of dPEG modified

$\left\{\mathrm{La}_{x} \mathrm{Gd}_{1-\mathrm{x}}\right\} \mathrm{PO}_{4} @ 4 \mathrm{LnPO}_{4} @$ Au nanoparticles was dispersed in $0.5 \mathrm{~mL}$ of PBS in a $1 \mathrm{~mL}$ microcentrifuge vial. To this vial, $8 \mu \mathrm{L}$ of $10 \mathrm{mg} / \mathrm{mL}$ sulfo-NHS and $80 \mu \mathrm{L}$ of $10 \mathrm{mg} / \mathrm{mL}$ EDC were added and stirred for 30 minutes. The solution was centrifuged for 3 minutes at $3000 \mathrm{~g}$ and the supernatant decanted via pipette to remove excess EDC/sulfo-NHS. The nanoparticles were redispersed in $0.5 \mathrm{~mL}$ of PBS and stirred with $0.5 \mathrm{mg}$ of antibody overnight $(\mathrm{pH}=7.4)$. The reaction was quenched with glycine and stirred for 15 minutes. Particles were again centrifuged as above and redispersed in $0.5 \mathrm{~mL}$ of PBS containing $5 \mathrm{mg} / \mathrm{mL}$ BSA. The mechanism for the amide reaction is shown in Figure

\subsection{2.}



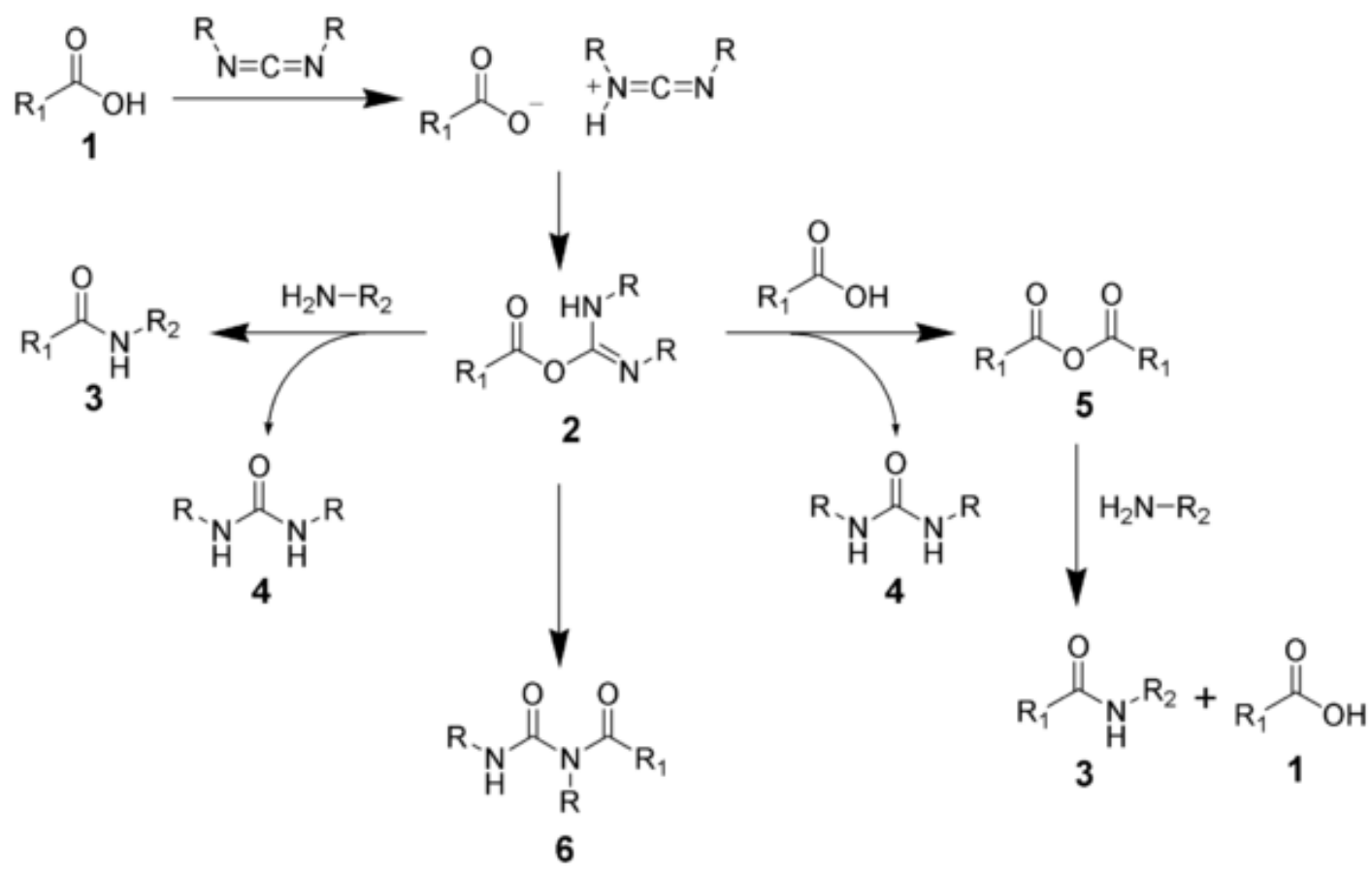

Figure 3.4.2: Amide bond formation by carbodiimide activation

\subsubsection{In Vitro Antibody Quantification and Competition Assay}

Panitumumab was added to the nanoparticles as in 3.4.3. Nanoparticles were centrifuged and washed with PBS 5 times before determining surface protein concentration via a modified Lowry assay. ${ }^{99}$ Briefly, eleven standards were created with varying amounts of BSA (0-20 $\mu \mathrm{g})$ for comparison purposes. Each polypropylene tube was filled with the appropriate amount of BSA, $10 \mu \mathrm{L}$ of PBS buffer, and diluted to 300 $\mu \mathrm{L}$ with DI water. Samples were created by taking 6,8 , or $10 \mu \mathrm{L}$ of nanoparticle solution, adding PBS to bring the volume to $10 \mu \mathrm{L}$, then diluting to a volume of $300 \mu \mathrm{L}$ with deionized water. Solution A was prepared by mixing $0.5 \mathrm{~mL}$ of $1 \%$ cupric sulfate and 0.5 $\mathrm{mL}$ of $2 \%$ potassium tartrate with a solution of $50 \mathrm{~mL}$ of $2 \%$ sodium carbonate/0.1 $\mathrm{M}$ 
$\mathrm{NaOH}$. Solution $\mathrm{A}(1.5 \mathrm{~mL})$ was added to each tube, vortexed, and allowed to stand at room temperature for 10 minutes. Next, $150 \mu \mathrm{L}$ of $1 \mathrm{M}$ phenol was added to each tube and immediately vortexed. The tubes were allowed to stand at room temperature for 30 minutes before measuring their absorbance at $750 \mathrm{~nm}$. Absorbance in the nanoparticle samples was compared to the absorbance of the BSA standard to determine protein concentration on the nanoparticles. Nanoparticles with PEG attached were treated similarly and used as controls for the antibody labeled nanoparticle samples. Absorbance from the PEG coated nanoparticles was subtracted from the absorbance of the antibody labeled nanoparticles. The number of antibodies per nanoparticle was calculated by assuming a $150 \mathrm{kDa}$ antibody and a nanoparticle consisting of a $22.4 \mathrm{~nm}$ diameter $\mathrm{LnPO}_{4}$ with a density of $5 \mathrm{~g} / \mathrm{cm}^{3}$ and a $0.5 \mathrm{~nm}$ thick outer layer of gold of density of $19.3 \mathrm{~g} / \mathrm{cm}^{3}$. An example calculation is given in Appendix 3.

Human epidermal growth factor receptor (hEGFR) was coated onto well plates (50 ng hEGFR/50 $\mu \mathrm{L}$ DPBS per well) and incubated overnight at $4{ }^{\circ} \mathrm{C}$. The following day, hEGFR was removed and each well washed with $100 \mu \mathrm{L}$ of $1 \%$ BSA in PBS. The wells were kept at room temperature for 1 hour before removal of PBS. A series of 11 dilutions of HuM-195, panitumumab, nanoparticle-HuM-195 conjugates, and nanoparticle-panitumumab conjugates with protein concentrations from $0.8 \mathrm{ng} / \mathrm{mL}-40$ $\mu \mathrm{g} / \mathrm{mL}$ (as determined by Lowry assay) was prepared. Twenty-five $\mu \mathrm{L}$ of each dilution and a control consisting of PBS was added to the wells in triplicate followed by addition 
of $25 \mu \mathrm{L}$ of panitumumab antibody labeled with $50,000 \mathrm{cpm}{ }^{131} \mathrm{I}$. The wells were incubated for 4 hours at $37^{\circ} \mathrm{C}$, then washed $3 x$ with $150 \mu \mathrm{L}$ of $1 \%$ BSA in PBS. One hundred $\mu \mathrm{L}$ of $0.2 \mathrm{M} \mathrm{NaOH}$ was added to each well to release the bound hEGFR. Wells were shaken for 2 minutes at a moderate speed and their contents absorbed onto filters and transferred into polypropylene tubes for counting. The amount of radioactivity absorbed onto the filters was then used to compare the binding activity of the nanoparticle-antibody conjugates to that of the free antibodies.

\subsubsection{Radiotracer Antibody Saturation Studies and Biodistribution}

MAb 201b was radioiodinated with ${ }^{125}$ I by the chloramine T method. ${ }^{100}$ Briefly, $15 \mu \mathrm{g}$ of antibody was added to $75 \mu \mathrm{L}$ of a freshly prepared solution of $50 \mathrm{mM} \mathrm{NaPO}_{4}$. $\mathrm{Na}^{125} \mathrm{I}(6 \mu \mathrm{L}, 3 \mathrm{mCi})$ was added to this solution followed by $75 \mu \mathrm{L}$ of $4 \mathrm{mg} / \mathrm{mL}$ chloramines $\mathrm{T}$ in $50 \mathrm{mM} \mathrm{NaPO}_{4}$. The solution was mixed with a stir bar for 30 seconds, then quenched by addition of $300 \mu \mathrm{L}$ of $12.6 \mathrm{mM}$ sodium metabisulfate in $50 \mathrm{mM}$ $\mathrm{NaPO}_{4}$. The quenched reaction was dialyzed 2 times versus PBS to remove unbound ${ }^{125} \mathrm{I}$. Three $500 \mu \mathrm{L}$ microcentrifuge tubes were set up containing 5, 55, or $250 \mu \mathrm{g}$ of mAb 201b. Each mAb addition contained $5 \mu \mathrm{g}$ of radiolabeled antibody and an appropriate amount of cold antibody to provide the correct total mAb mass in a final volume of 100 $\mu \mathrm{L}$ in PBS. Ten $\mu \mathrm{L}$ of $0.5 \mathrm{M}$ sodium phosphate buffer, $\mathrm{pH} 7.6$ was added to each tube. 
Nanoparticles (1.5 mg in $600 \mu \mathrm{L}$ of PBS) were activated as described in 3.4.3. Two hundred $\mu \mathrm{L}$ of this solution was dispensed into each of 3 tubes containing antibody.

The mixture was allowed to react overnight, then quenched by addition of $5 \mu \mathrm{L}$ of $1 \mathrm{M}$ glycine and stirred for 1 hour. The particles were centrifuged as above and the supernatant sampled for ${ }^{125} \mathrm{I}$ content. The number of antibodies per nanoparticle was quantified by calculating the average mass of an individual particle assuming an inner $\mathrm{LnPO}_{4}$ shell with a $22.4 \mathrm{~nm}$ diameter and a density of $5 \mathrm{~g} / \mathrm{cm}^{3}$ and a $0.5 \mathrm{~nm}$ thick outer layer of gold with a density of $19.3 \mathrm{~g} / \mathrm{cm}^{3}$.

Nanoparticles were centrifuged again, resuspended in $0.5 \mathrm{~mL}$ of $5 \mathrm{mg} / \mathrm{mL} \mathrm{BSA}$ in PBS and sonicated (Branson microprobe) for 2-5 sec to complete dispersal. Another 0.5 $\mathrm{mL}$ of BSA/PBS buffer was added to each preparation and $200 \mu \mathrm{L}$ of the three preparations injected via the tail vein into 4 female BALB/c mice (body mass $\sim 20 \mathrm{~g}$ ) per group for a total of 12 mice. Mice (2 per group) were sacrificed at $1 \mathrm{hr}$ or $24 \mathrm{hrs}$ postinjection and liver, spleen, kidneys and lung were harvested for biodistribution studies. Each organ was counted for ${ }^{125} \mathrm{I},{ }^{221} \mathrm{Fr}$, and ${ }^{213} \mathrm{Bi}$ content at $\sim 20$ minutes post sacrifice and then again after ${ }^{213} \mathrm{Bi}$ equilibrium had been established $3 \mathrm{hrs}$ post-sacrifice.

\subsubsection{Biodistribution and in vivo Retention Studies with $\left\{\mathrm{La}_{0.5} \mathrm{Gd}_{0.5}\right\} \mathrm{PO}_{4} @ 4 \mathrm{GdPO} \mathrm{A}_{4} @ \mathrm{Au}-$} PEG-mAb 201b

Biodistribution and ${ }^{225} \mathrm{Ac}$ daughter retention assays were done on three separate groups consisting of six female BALB/c mice per group, injected I.V. (tail vein). Groups 1 
and 2 were injected with $\left\{\mathrm{La}_{0.5} \mathrm{Gd}_{0.5}\right\} \mathrm{PO}_{4} @ 4 \mathrm{GdPO}{ }_{4} @ A u-P E G-m A b 201 \mathrm{~b}$, while group 3 was treated with $\left\{\mathrm{La}_{0.5} \mathrm{Gd}_{0.5}\right\} \mathrm{PO}_{4} @ 4 \mathrm{GdPO}_{4} @ A u-\mathrm{PEG}$ nanoparticles as a control. Group 1 mice received $14.6 \mu \mathrm{g}$ of conjugates with $74 \mathrm{kBq}$ of Ac-225 and $\sim 5 \mathrm{~g}$ of attached mAb $201 b$ (as measured in 3.4.5) in $100 \mu \mathrm{L}$ of PBS. Group 2 received an identical amount of targeted nanoparticles but with co-injection of $750 \mu \mathrm{g}$ of unconjugated mAb 201b as

competitor. Group 3 received the same amount of nanoparticles and ${ }^{225} \mathrm{Ac}$, but with no conjugated antibody. Mice received food and water ad libitum in a light/dark cycle environment. Three mice from each injection group were sacrificed at time points of 1 and 24 hours post-injection for biodistribution and in vivo retention studies. Mouse lung, liver, spleen, and kidneys were harvested to evaluate the amount of both ${ }^{221} \mathrm{Fr}$ and ${ }^{213} \mathrm{Bi}$ in target organs. Each organ was massed and counted for content of ${ }^{221} \mathrm{Fr}$ and ${ }^{213} \mathrm{Bi}$ in a $y$ scintillation counter at a specific time post sacrifice and again after the radioisotopes had achieved decay equilibrium (>3 h). Quantities of ${ }^{221} \mathrm{Fr}$ and ${ }^{213} \mathrm{Bi}$ present at the time of animal sacrifice were determined by appropriate decay corrections as previously described. ${ }^{48}$

\subsubsection{MicroSPECT/CT Imaging:}

Small animal imaging was performed using a Siemens Preclinical Imaging microCAT IIP SPECT dual modality platform on loan to the University of Tennessee. Mice were injected I.V. with approximately $3 \mathrm{MBq}$ of ${ }^{225} \mathrm{Ac}$ in the same three groups and with the same volumes as in 3.4.6. Instead of the large excess described in 3.4.6, an identical quantity of free antibody was injected in group 2, thus the mice in the 
competition group had a different ratio of cold competitor to nanoparticle conjugate and competition was not as complete. Animals were sacrificed by overdose of isoflurane at 1 hour post injection and imaged via microSPECT/CT 3 hours post-sacrifice when the ${ }^{225} \mathrm{Ac}$ and its daughters had reached equilibrium. SPECT data for the final images were acquired as previously described. ${ }^{48}$

\subsubsection{Effect of Clodronate Liposomes on Biodistribution and Uptake}

Biodistribution and daughter retention assays were done using I.V. injections in four groups of mice, consisting of six mice per group. Mice received $14.6 \mu \mathrm{g}$ of $\left\{\mathrm{La}_{0.5} \mathrm{Gd}_{0.5}\right\} \mathrm{PO}_{4} @ 4 \mathrm{GdPO}{ }_{4} @ A u-P E G-m A b 201 \mathrm{~b}$ nanoparticle conjugates with 74 kBq of ${ }^{225} \mathrm{Ac}$ and $\sim 5 \mu \mathrm{g}$ of attached mAb 201b in $100 \mu \mathrm{L}$ of PBS. Group 1 was injected with clodronate liposomes followed by nanoparticle-mAb 201b conjugates. Mice in group 2 were injected with clodronate liposomes followed by a mixture of $500 \mu \mathrm{g}$ of unconjugated mAb 201b and nanoparticle-mAb 201b conjugates. Group 3 and 4 mice were injected as group 1 and 2, respectively, except that they were not pretreated with clodronate liposomes. Mice were housed with food and water ad libitum in a light/dark cycle environment. Three mice from each injection group were sacrificed at 1 and 24 hours post injection for biodistribution and in vivo retention studies. A y scintillation counter windowed for $212 \mathrm{keV}\left({ }^{221} \mathrm{Fr}\right)$ and $440 \mathrm{keV}\left({ }^{213} \mathrm{Bi}\right)$ was used to evaluate the amount of radioactivity present in the harvested lungs, liver, spleen, and kidneys. Quantities of ${ }^{221} \mathrm{Fr}$ and ${ }^{213} \mathrm{Bi}$ present at the precise time of animal sacrifice were determined by appropriate crossover and decay corrections as previously described. ${ }^{48}$ 


\subsubsection{Electron Microscopy Samples}

Mice were anesthetized with an oxygen/isoflurane mixture. Each mouse received $100 \mu \mathrm{L}$ of $\left\{\mathrm{La}_{0.5} \mathrm{Gd}_{0.5}\right\} \mathrm{PO}_{4} @ 4 \mathrm{GdPO} \mathrm{A}_{4} @ \mathrm{Au}-\mathrm{PEG}-\mathrm{mAb} 201 \mathrm{~b}$ non-radioactive conjugates prepared as described in 3.4.3. After 45 minutes, each mouse was sacrificed by injection of $4 \mathrm{~mL}$ of fixative buffer solution ( $2 \%$ paraformaldehyde, $2 \%$

glutaraldehyde, $0.1 \mathrm{M}$ cacodylate, hereafter referred to as simply cacodylate) via the catheter as below and euthanized via cervical dislocation. Mouse tissues were embedded in resin, sliced, and fixed for examination on CU TEM grids (Ted Pella). Electron microscopy studies were performed at the University of Missouri Electron Microscopy Core on a JEOL 1400 instrument.

\subsubsection{EMT-6 Therapy Study}

Fifteen mice were injected I.V. (tail vein) with clodronate liposomes followed the next day by injection of 80,000 log phase EMT- 6 cells in $0.2 \mathrm{~mL}$ of PBS. EMT-6 cells form tumor colonies in lung tissue simulating metastatic disease. ${ }^{101}$ Forty-eight hours after tumor cell injection, 5 mice were injected with $100 \mu \mathrm{L}$ of PBS, 5 mice with free mAb 201b mixed with 37 kBq of $\left\{\mathrm{La}_{0.5} \mathrm{Gd}_{0.5}\right\} \mathrm{PO}_{4} @ 4 \mathrm{GdPO}{ }_{4} @ A u-P E G-m A b$ 201b conjugates, and 5 mice with $\sim 37 \mathrm{kBq}$ of nanoparticle-mAb 201B conjugates. Mice were sacrificed at 7 days post EMT-6 cell injection. Lungs were inflated via tracheal catheter with Bouin's fixative ( $0.2 \%$ picric acid, $2 \%$ paraformaldehyde, $40 \%$ formalin in water), suspended in fixative for 24 hours and then washed into 70\% ethanol/PBS. Paraffin sections were cut, 
stained, and counted by a blinded observer to quantitate tumor colony growth. Statistical significance was calculated at the $95 \%$ confidence level using a two tailed Student's T test with unequal variance. Separate samples were fixed in cacodylate buffer and allowed to decay for 6 months before examination on electron microscopy.

\subsubsection{In Vivo Evaluation of Blood Brain Barrier Penetration with Anti-GFAP and Anti-}

\section{Synaptophysin}

Anti-GFAP and anti-synaptophysin antibodies were added to $\left\{\mathrm{La}_{0.5} \mathrm{Gd}_{0.5}\right\} \mathrm{PO}_{4} @ 4 \mathrm{GdPO}_{4} @ A u-P E G$ nanoparticles as described in 3.4.3. The attachment of the antibodies to the nanoparticles was confirmed with immunohistochemistry. The anti-synaptophysin and anti-GFAP antibody labeled nanoparticles were incubated with normal brain and used as a primary immunochemistry label to target these proteins in normal brain. Secondary antibody biotin and avidin-horseradish peroxidase were used to detect the presence of primary antibody. Diaminobenzadine (DAB) was used as the color agent.

Mice were anesthetized with an isoflurane (1.5\%) and oxygen mixture and catheterized via partial dissection in either the femoral or axillary vein using a $0.115 \mathrm{~mm}$ outer diameter, $0.10 \mathrm{~mm}$ inner diameter catheter (A.P. Extrusion, Salem, N.H.). Mannitol $(50 \mu \mathrm{L})$ was injected to open the blood-brain barrier. One hundred $\mu \mathrm{L}(300 \mu \mathrm{g})$ of immunolabeled nanoparticles (anti-synaptophysin or anti-GFAP) were injected intravenously into each mouse. Under terminal anesthesia, mice were sacrificed by 
perfusion cacodylate buffer 30 minutes post-injection. Death was ensured via cervical dislocation. The brain, tongue, colon, muscle, heart, spleen, kidney, lung, and liver were harvested. The tissue was prepared for analysis by TEM, light microscopy, and NAA.

\subsection{Biological Targeting Results and Discussion}

\subsubsection{Characterization of Antibody Attachment}

The absorption wavelength of the plasmon resonance as measured by UV-Vis spectroscopy steadily increases as larger groups are added to the nanoparticle surface. Citrate coated $\left\{\mathrm{La}_{0.5} \mathrm{Gd}_{0.5}\right\} \mathrm{PO}_{4} @ 4 \mathrm{GdPO}_{4} @$ Au nanoparticles absorb maximally at $523 \mathrm{~nm}$. After addition of the lipoamide- $\mathrm{PPEG}_{12}-\mathrm{COOH}$ linker, this absorption peak red-shifts to $529 \mathrm{~nm}$. After antibody addition, the plasmon peak further red-shifts to $537 \mathrm{~nm}$. The changes in plasmon resonance for the addition of the anti-synaptophysin antibody are shown in Figure 3.5.1.

The antibody addition process also causes a drastic increase in the hydrodynamic diameter of the nanoparticles. From an initial hydrodynamic diameter of $101.4 \pm 1.5$ $\mathrm{nm}$, addition of mAb $201 \mathrm{~b}$ causes it to grow to $1498 \pm 77 \mathrm{~nm}$. Zeta potential also becomes more positive as a result of mAb $201 \mathrm{~b}$ addition. The nanoparticle surface has been passivated by the addition of PEG and $\mathrm{mAb}$, so that the nanoparticles will not aggregate despite the more neutral zeta potential. 


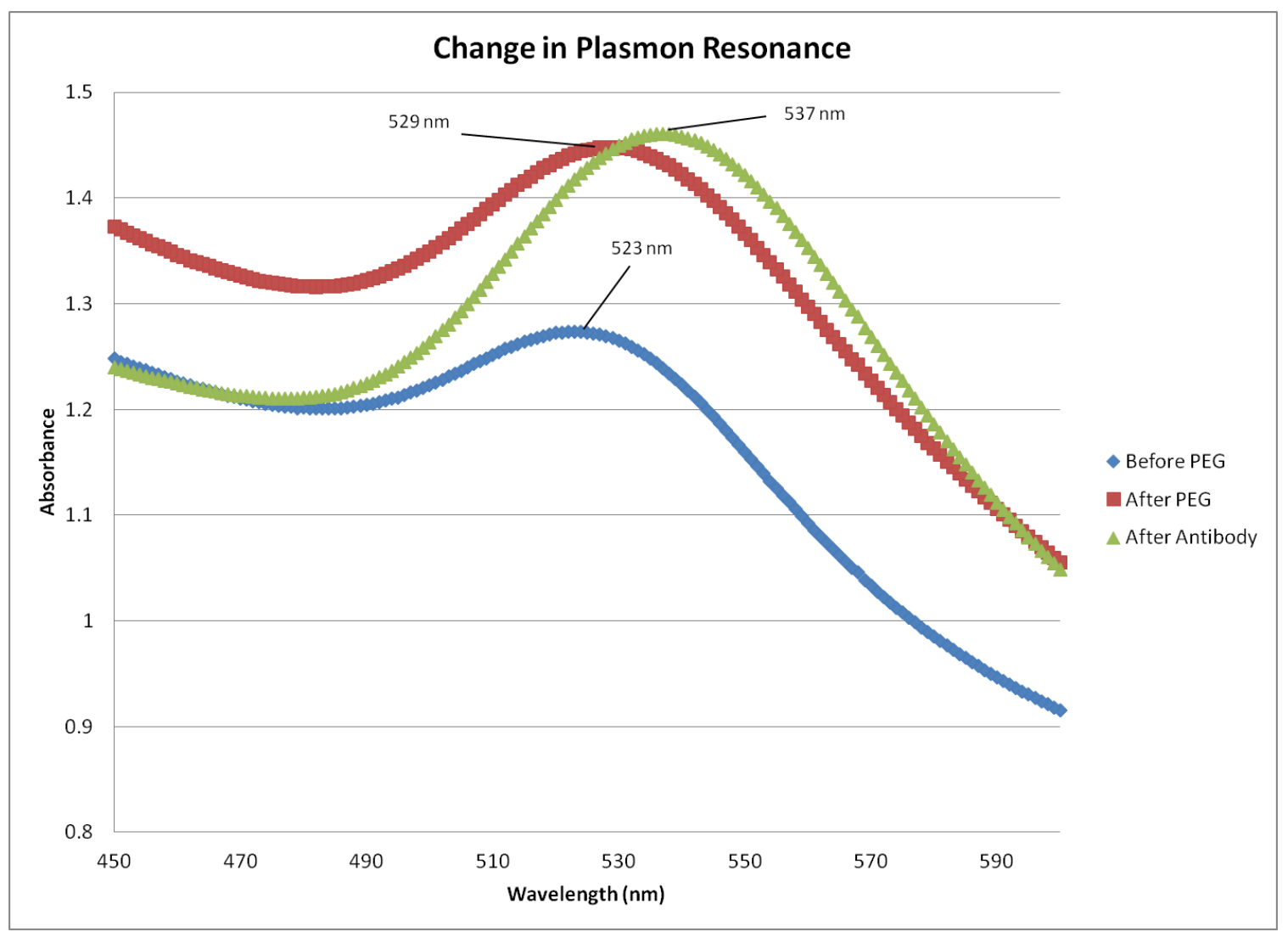

Figure 3.5.1: UV-Vis spectra showing the red-shift in the plasmon resonance as PEG and antibody are added to citrate coated $\left\{\mathrm{La}_{0.5} \mathrm{Gd}_{0.5}\right\} \mathrm{PO}_{4} @ 4 \mathrm{GdPO}_{4} @ A u$ nanoparticles.

Table 3.5.1: Hydrodynamic diameter and zeta potential of $\left\{\mathrm{La}_{0.5} \mathrm{Gd}_{0.5}\right\} \mathrm{PO}_{4} @ G \mathrm{GPO}{ }_{4} @ A u-$ mAb-201b nanoparticles.

\begin{tabular}{|c|c|c|}
\hline Nanoparticle composition & $\begin{array}{l}\text { Hydrodynamic } \\
\text { diameter (nm) }\end{array}$ & $\begin{array}{l}\text { Zeta potential } \\
(\mathrm{mV})\end{array}$ \\
\hline$\left\{\mathrm{La}_{0.5} \mathrm{Gd}_{0.5}\right\} \mathrm{PO}_{4} @ \mathrm{GdPO} \mathrm{O}_{4} @$ Au-citrate & $101.4 \pm 1.5$ & $-63.2 \pm 1.6$ \\
\hline$\left\{\mathrm{La}_{0.5} \mathrm{Gd}_{0.5}\right\} \mathrm{PO}_{4} @ \mathrm{GdPO}_{4} @ \mathrm{Au}-\mathrm{PEG}$ & $382.3 \pm 6.5$ & $-56.4 \pm 0.1$ \\
\hline$\left\{\mathrm{La}_{0.5} \mathrm{Gd}_{0.5}\right\} \mathrm{PO}_{4} @ \mathrm{GdPO}_{4} @ A u-m A b-201 b$ & $1498 \pm 77$ & $-27.9 \pm 2.4$ \\
\hline
\end{tabular}


The Lowry assay detected $0.22 \mathrm{mg}$ of HuM-195 and $0.19 \mathrm{mg}$ of panitumumab bound to the nanoparticles. This quantity compares with $0.875 \mathrm{mg}$ of each antibody which was used in the synthesis, giving a labeling yield of $25.1 \%$ for HuM-195 and $21.7 \%$ for panitumumab. These labeling yields correspond to an average of $4.9 \mathrm{HuM}-195$ antibodies per nanoparticle and an average of 4.3 panitumumab antibodies per nanoparticle.

Nanoparticle-panitumumab conjugates show binding characteristics similar to the panitumumab antibody. However, the binding affinity of the conjugates, as measured by the $\mathrm{IC}_{50}$ value, appears to be roughly $4.5 x$ lower than the free antibody. This difference is likely explained by the loading of multiple antibodies onto the nanoparticle. Binding to hEGFR will only occur through one of the average 4.3 antibodies on the nanoparticle surface. The other antibodies remain, but do not take up receptor sites, hence the apparent decrease in binding affinity. Correcting for this factor brings the binding affinity of the nanoparticle-panitumumab conjugate in line with the free panitumumab antibody. The conjugation process therefore does not decrease the inherent binding ability of the antibody. HuM-195 and nanoparticle-Hum 195 conjugate controls show no affinity for the hEGFR receptor at any concentration. Results from the competition assay are shown in Figure 3.5.2.

Antibody conjugation was also quantified using a ${ }^{125} \mathrm{I}$ radiotracer to label mAb 201b prior to attachment. MAb 201b has high affinity and specificity for thrombomodulin in vivo, which is expressed primarily in lung tissue. Actinium-225 was 
recovered nearly quantitatively in these antibody saturation experiments, indicating little loss of nanoparticles. Attachment of mAb 201b varied with amount added according to table 3.5.2. The amount of antibody bound approaches a maximum, indicating saturation of the available sites at slightly over 4 antibodies per nanoparticle. The maximum antibody bound per particle corresponds well with the labeling yield seen with panitumumab and HuM-195.

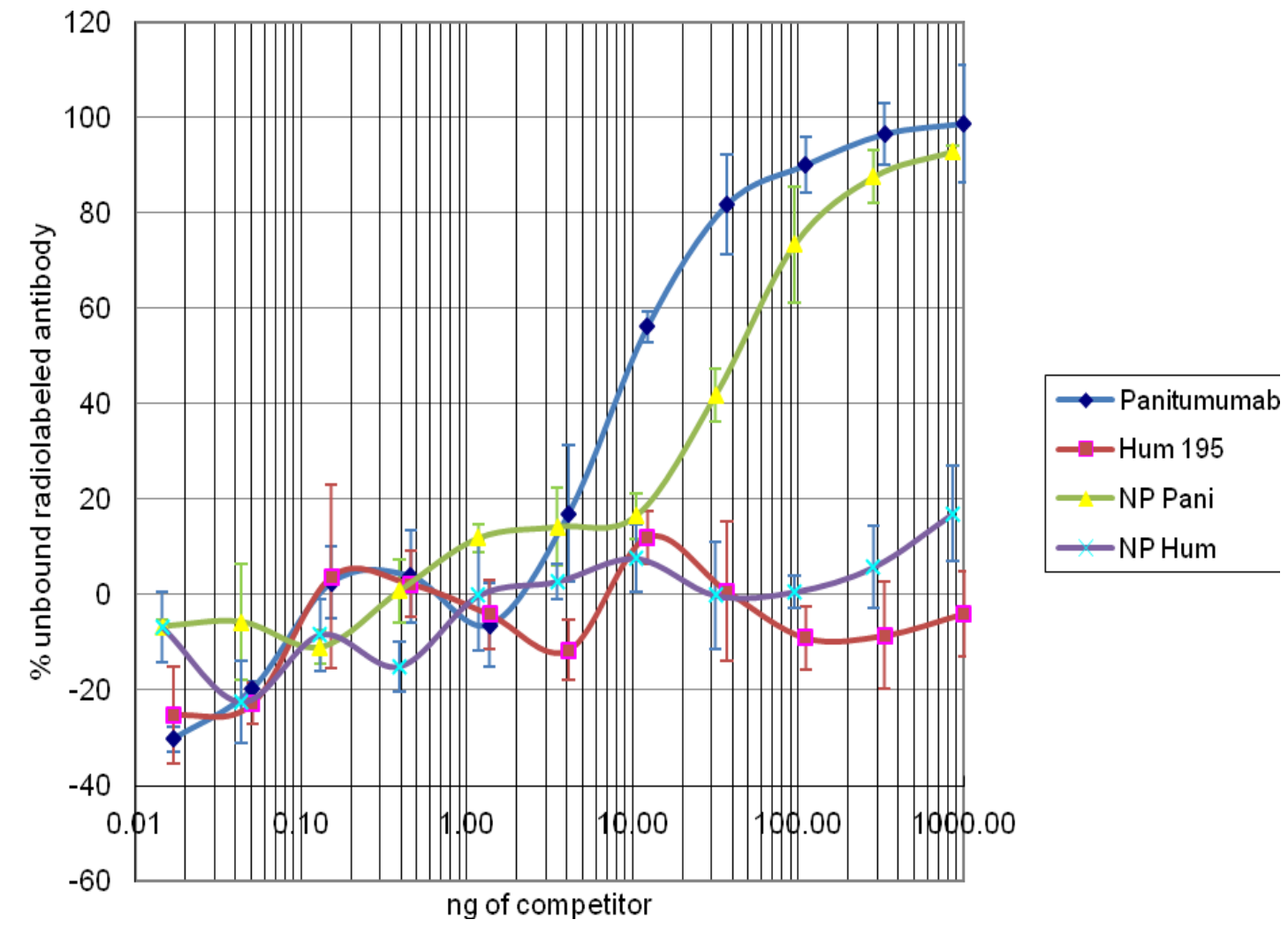

Figure 3.5.2: Nanoparticle-panitumumab conjugates show a decreased binding affinity relative to free antibody due to the binding of multiple panitumumab molecules onto a single nanoparticle. Nanoparticle-Hum 195 conjugates and free Hum 195 antibody show no affinity for the hEGFR receptor used at any concentration. 
Table 3.5.2: Labeling efficiency of mAb $201 \mathrm{~b}$ onto $0.5 \mathrm{mg}$ of $\left\{\mathrm{La}_{0.5} \mathrm{Gd}_{0.5}\right\}\left({ }^{225} \mathrm{Ac}\right) \mathrm{PO}_{4} @ 4$ $\mathrm{GdPO}_{4}$ shell@Au nanoparticles

\begin{tabular}{|l|l|l|l|}
\hline mAb added $(\mu \mathrm{g})$ & mAb bound $(\mu \mathrm{g})$ & $\%$ bound & mAb/NP \\
\hline 5 & 4.5 & 90 & 0.65 \\
\hline 55 & 26.0 & 48 & 3.39 \\
\hline 255 & 32.2 & 13 & 4.20 \\
\hline
\end{tabular}

\subsubsection{Lung Targeting with mAb 201b}

Localization of activity in the lung at 1 hour depends on the amount of antibody used in the preparation. Nanoparticles with a larger amount of antibody bound show higher uptake by lung tissue. This effect remains evident after 24 hours, as shown in Figure 3.5.3.

The \%ID of each radionuclide detected in lung tissue does not match. This indicates that the ${ }^{125} \mathrm{I}$ and ${ }^{225} \mathrm{Ac}$ daughter products are becoming separated in vivo. Further, the ratio of \%ID ${ }^{225} \mathrm{Ac}$ to $\% \mathrm{ID}^{125} \mathrm{I}$ detected in depends both on the amount of antibody loaded onto the individual particles and the time since injection. Over a 24 hour period, the ratio increases by roughly a factor of 5 . Those ratios are shown in Table 3.5.3. 


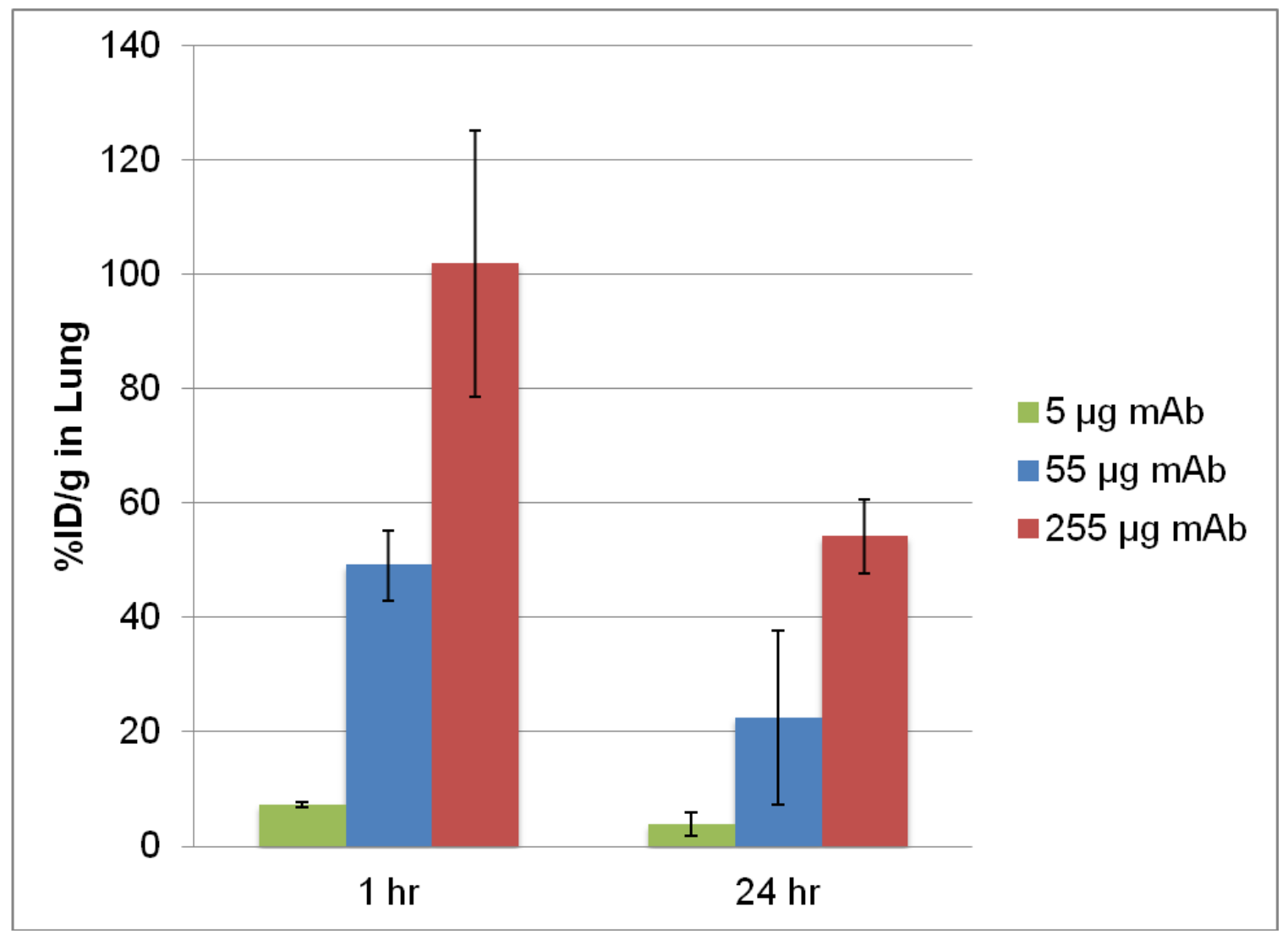

Figure 3.5.3: Saturating the nanoparticle with antibody increases targeting and retention in lung tissue at time points of 1 hour and 24 hours. Percent ID/g increases as a function of the amount of antibody used in the synthesis at both time points.

Table 3.5.3: Ratio of \%ID/g ${ }^{225} \mathrm{Ac}$ to $\% \mathrm{ID} / \mathrm{g}^{125} \mathrm{I}$ in lung tissue at 1 hour and 24 hours

\begin{tabular}{|l|l|l|}
\hline antibody added $(\mu \mathrm{g})$ & $1 \mathrm{~h}$ & $24 \mathrm{~h}$ \\
\hline 5 & 0.15 & 1.50 \\
\hline 55 & 0.97 & 5.45 \\
\hline 250 & 1.08 & 4.47 \\
\hline
\end{tabular}


There are a variety of explanations for the discrepancy between $\%$ ID ${ }^{225} \mathrm{Ac}$ and $\%$ ID ${ }^{125}$ I that present a complicated picture of in vivo interactions. When the amount of antibody on each particle is low, the ratio of \%ID of ${ }^{225}$ Ac to \%ID of ${ }^{125}$ I at one hour is 0.15 , indicating that some nanoparticle must be cleaved from antibody and leave the lung. This ratio with moderate and high antibody loading onto the nanoparticles is close to unity at 1 hour, indicating that the radiolabeled antibody and nanoparticle are remaining intact. More than one antibody may bind the nanoparticle to the lung, stabilizing the attachment. This interpretation is consistent with the number of antibodies bound to each nanoparticle. If more than one antibody is present per nanoparticle, cooperative binding ensures that the nanoparticle remains attached to the target site.

At $24 \mathrm{hrs},{ }^{125} \mathrm{I}$ is nearly gone in all samples. ${ }^{225} \mathrm{Ac}$ is also lost, but at a drastically slower rate. The loss of ${ }^{125} \mathrm{I}$ is also much faster than loss of the free antibody. ${ }^{100}$ The increase of \%ID ${ }^{225} \mathrm{Ac}$ relative to $\%$ ID ${ }^{125} \mathrm{I}$ from the $1 \mathrm{~h}$ to $24 \mathrm{~h}$ time point indicates that the nanoparticles are being retained in the lung tissue while either the targeting antibody or the ${ }^{125}$ I label separates from the nanoparticle. lodine-125 linked by the chloramines T method will cleave once inside the cellular environment and release into circulation. ${ }^{102}$ TEM images taken of the lung show the presence of nanoparticles in the lung epithelium (Figure 3.5.4), indicating that the change in ratio likely results from cleavage of ${ }^{125}$ I from the antibody. Other tissues show similar changes in the \%ID ratio 99 
from 1 to 24 hours indicating that the change results primarily from the loss of ${ }^{125} \mathrm{I}$. This could be confirmed by measuring the thyroid for escaped ${ }^{125} \mathrm{I}$.

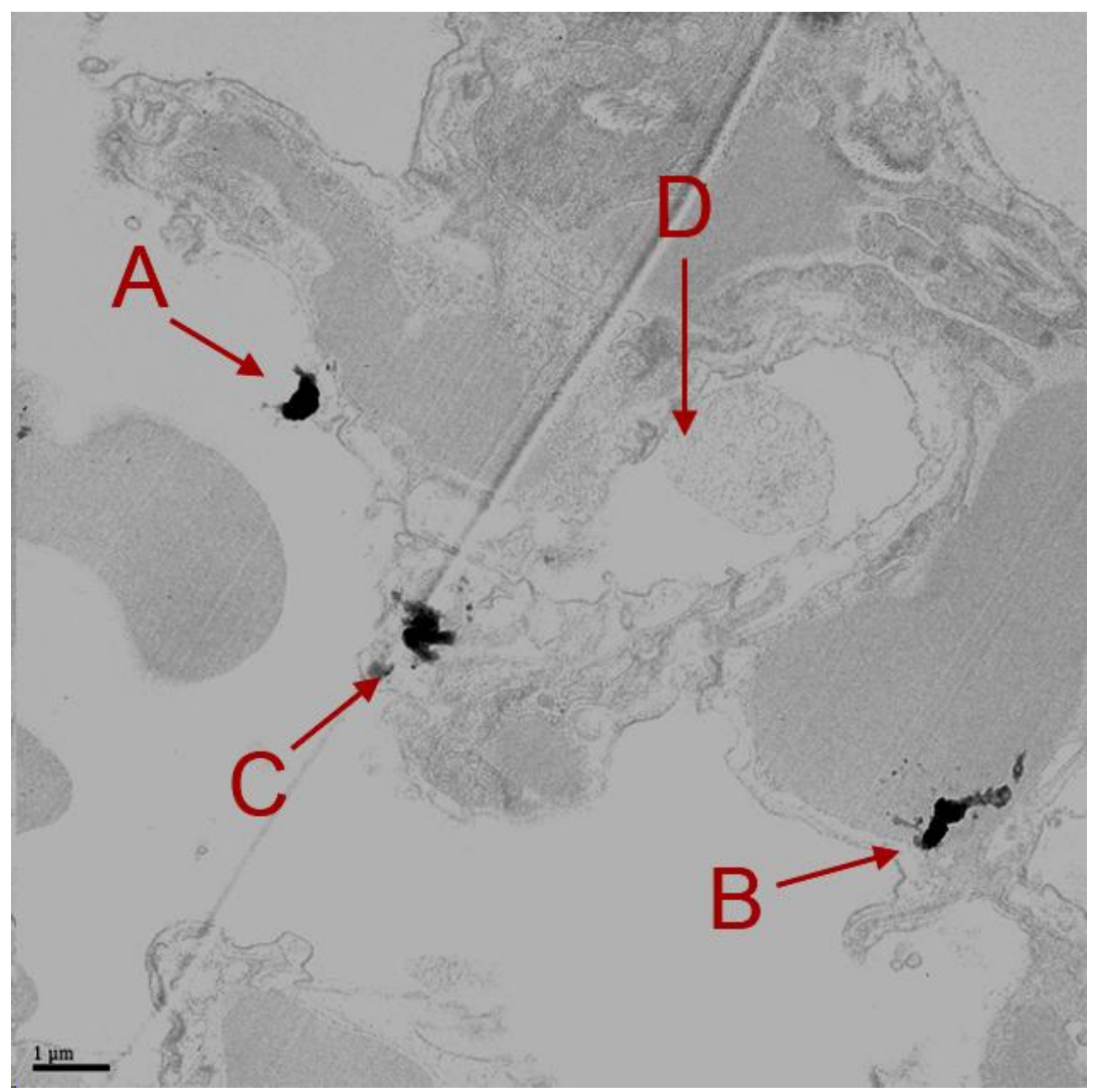

Figure 3.5.4: $\left\{\mathrm{Gd}_{0.5} \mathrm{La}_{0.5}\right\} \mathrm{PO}_{4} @ \mathrm{GdPO}_{4} @ A u$ nanoparticles bound to the vascular side of lung epithelium (A), contiguous with red blood cells (B), and actively being endocytosed (C) into a lung epithelium vesicle (D). 

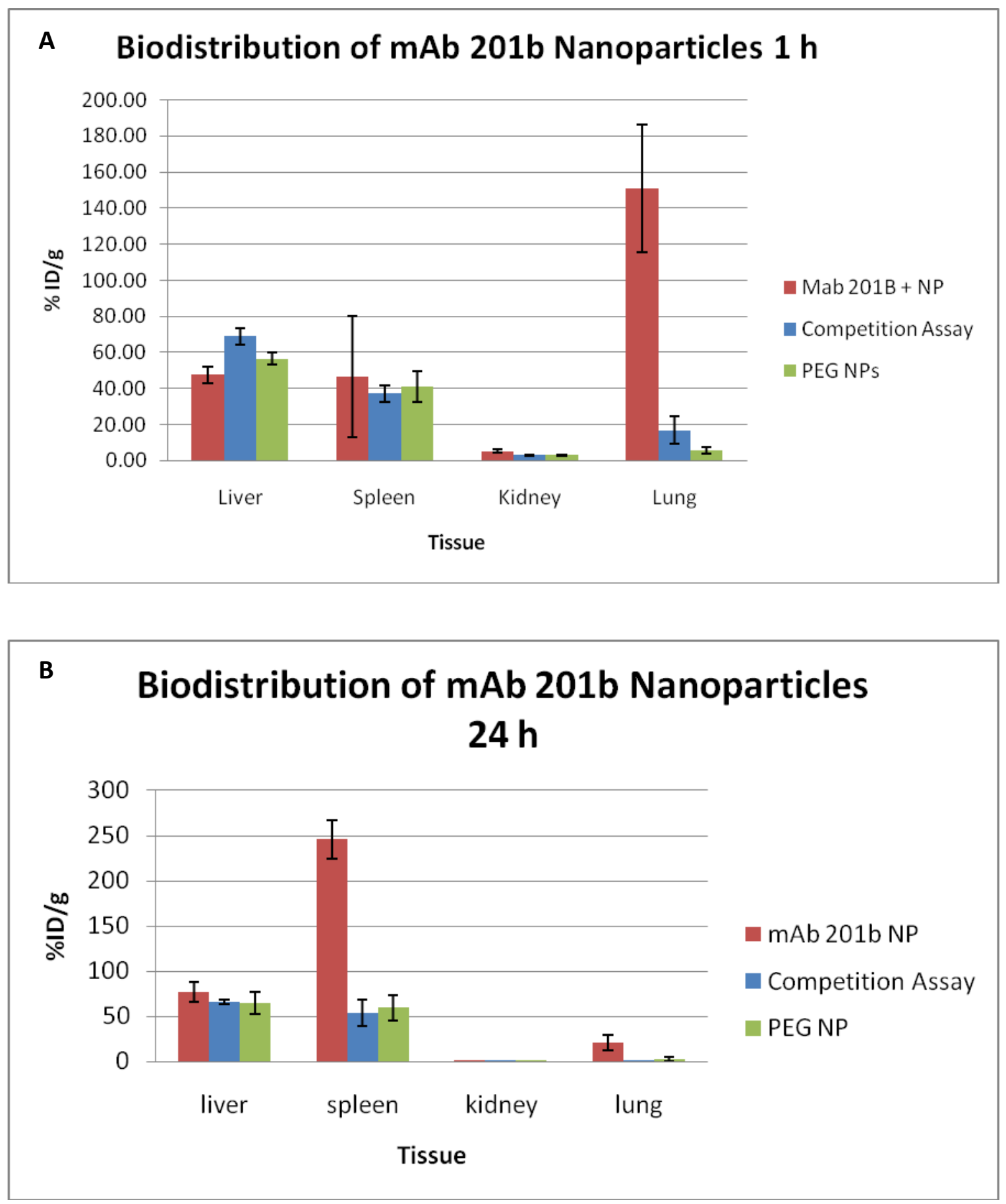

Figure 3.5.5 A and B: Biodistribution of $\left\{\mathrm{Gd}_{0.5} \mathrm{La}_{0.5}\right\} \mathrm{PO}_{4} @ 4 \mathrm{GdPO}_{4}$ shell@Au-PEG-mAb 201b nanoparticles at 1 hour post-injection (A) and 24 hours post-injection (B). 
Given the increases in lung uptake with additional amounts of antibody, $\left\{\mathrm{Gd}_{0.5} \mathrm{La}_{0.5}\right\} \mathrm{PO}_{4} @ 4 \mathrm{GdPO}_{4}$ shell@Au and $\left\{\mathrm{Gd}_{0.25} \mathrm{La}_{0.75}\right\} \mathrm{PO}_{4} @ 4 \mathrm{LaPO}_{4}$ shell@Au nanoparticles were saturated with mAb 201b for biodistribution studies by combination of equal masses of mAb and nanoparticles during synthesis. Targeted nanoparticles in each case were compared with non-targeted, PEG coated nanoparticles and with receptor-blocked mice. Biodistribution results are shown in Figure 3.5.5 A and B at time points of 1 and 24 hours.

In vivo biodistribution experiments of the $\left\{\mathrm{Gd}_{0.5} \mathrm{La}_{0.5}\right\}\left({ }^{225} \mathrm{Ac}\right) \mathrm{PO}_{4} @ 4 \mathrm{GdPO}_{4}$ shell@Au-PEG-mAb 201b nanoparticles demonstrated uptake in the lung consistent with the binding properties of mAb 201b. The nanoparticles exhibit high lung uptake with the antibody conjugate after 1 hour (151\%ID/g). This high lung uptake of nanoparticles drops to $16.8 \% \mathrm{ID} / \mathrm{g}$ when competed with unconjugated antibody. Thus, the antibody retains its binding affinity and specificity even after conjugation to the nanoparticles. Further, nanoparticle uptake in the lung is primarily through antibodymediated binding.

In vivo, $69 \% \pm 3 \%$ of the ${ }^{213} \mathrm{Bi}$ from the ${ }^{225} \mathrm{Ac}$ generator was retained in lung tissue after 1 hour and $84 \% \pm 3 \%$ was retained after 24 hours. Similar ${ }^{213} \mathrm{Bi}$ retention values were observed in liver ( 1 hour, $81 \% \pm 4 \% ; 24$ hours, 92\% $\pm 1 \%$ ) and spleen tissue (1 hour, $72 \% \pm 3 \%$; 24 hours, $82 \% \pm 16 \%$ ). In contrast to ${ }^{225}$ Ac conjugates made from metal chelation, a very small fraction of the injected dose travels to the kidney. Under $5 \%$ of the ID/g of the nanoparticles locate in the kidney after 1 hour and less than $1 \%$ after 24 102 
hours. Renal toxicity associated with ${ }^{213} \mathrm{Bi}$ relocation to the kidney typically forms the dose-limiting factor in ${ }^{225} \mathrm{Ac} \alpha$-generator therapies, ${ }^{103}$ however with $\left\{\mathrm{Gd}_{0.5} \mathrm{La}_{0.5}\right\}\left({ }^{225} \mathrm{Ac}\right) \mathrm{PO}_{4} @ 4 \mathrm{GdPO}_{4}$ shell@Au-PEG-mAb 201b nanoparticles only $2.8 \%$ of the ${ }^{213} \mathrm{Bi}$ from the injected dose migrated to kidney tissues after 1 hour. After 24 hours, this number further decreased to $1.5 \%$.

A larger dose (ca. 3.0 MBq/animal) of ${ }^{225} \mathrm{Ac}$ NPs was imaged using CT/SPECT of the ${ }^{221} \operatorname{Fr} \gamma$ ray $(218 \mathrm{keV}, 11.6 \%)$. The CT/SPECT images (Figure 3.5.6) clearly show large uptake in the lung for the $\left\{\mathrm{La}_{0.5} \mathrm{Gd}_{0.5}\right\}\left({ }^{225} \mathrm{Ac}\right) \mathrm{PO}_{4} @ \mathrm{GdPO}{ }_{4} @ A u-m A b-201 b$ nanoparticles which is in agreement with the biodistribution data. When competed with unconjugated mAb 201b antibody, the images reflect the higher RES uptake in the liver and spleen. Unconjugated, PEG-coated nanoparticles also show high uptake by the RES, further indicating that the lung uptake is antibody-mediated and not due to particulate trapping in the small capillary system of the lung.

Compared with single $\alpha$-emitting therapies, the use of in vivo $\alpha$ generators holds the potential to deliver a much larger biologically effective dose to target tissues. The high, receptor-mediated uptake of nanoparticles in the lung epithelium demonstrates the ability of $\left\{\mathrm{La}_{0.5} \mathrm{Gd}_{0.5}\right\}\left({ }^{225} \mathrm{Ac}\right) \mathrm{PO}_{4} @ \mathrm{GdPO} \mathrm{O}_{4} @$ Au nanoparticles to deliver ${ }^{225} \mathrm{Ac}$ to a tissue target in the vascular space. The nanoparticle construct improves ${ }^{225} \mathrm{Ac}$ daughter retention relative to both chelate approaches and previous nanoparticle systems both in vitro and in vivo. In vivo, the increase of retention of ${ }^{213} \mathrm{Bi}$ in the target tissue over time results from a combination the intrinsic retention of the nanoparticle and its 


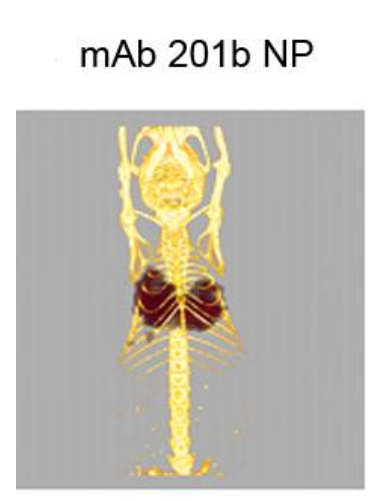

mAb 201b NP with competition
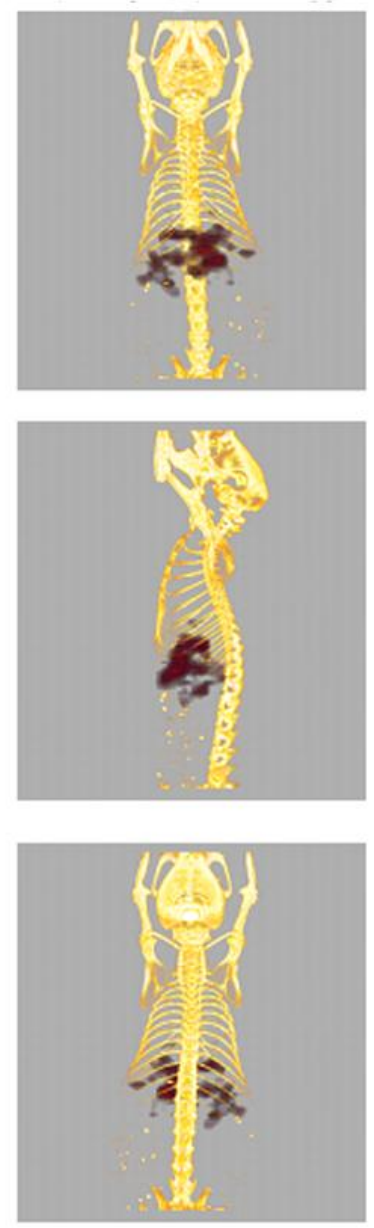

untargeted NP
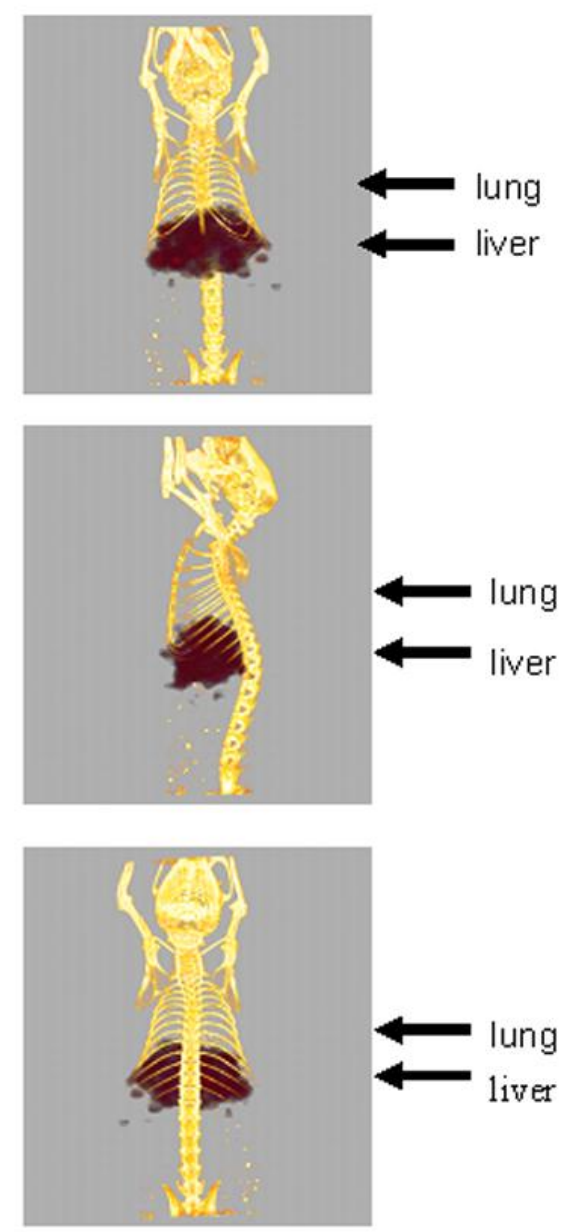

Figure 3.5.6: CT-SPECT scans of $\left\{\mathrm{La}_{0.5} \mathrm{Gd}_{0.5}\right\}\left({ }^{225} \mathrm{Ac}\right) \mathrm{PO}_{4} @ G d P \mathrm{O}_{4} @ A u-m A b-201 b$ show high uptake in the lung when targeted (left column). When competed with mAb-201b (middle column) and when untargeted (right column), uptake occurs in the RES.

endocytosis into lung epithelium. Daughters later in the decay series, such as ${ }^{213} \mathrm{Bi}$, are harder to retain because prior decays can move the remaining $\alpha$-emitting nuclides out of the nanoparticle core towards the nanoparticle surface. From this position nearer the surface, subsequent $\alpha$ decays are more likely to release the daughter nuclide from 
the nanoparticle. Thus, the retention values for ${ }^{221} \mathrm{Fr}$ should be higher than those for ${ }^{213} \mathrm{Bi}$. The amount of ${ }^{213} \mathrm{Bi}$ which relocates to the kidney from other tissues shows marked improvement with the $\left\{\mathrm{La}_{0.5} \mathrm{Gd}_{0.5}\right\}\left({ }^{225} \mathrm{Ac}\right) \mathrm{PO}_{4} @ \mathrm{GdPO}{ }_{4} @ \mathrm{Au}-\mathrm{mAb}-201 \mathrm{~b}$ system compared to core only nanoparticles. Layered $\left\{\mathrm{La}_{0.5} \mathrm{Gd}_{0.5}\right\}\left({ }^{225} \mathrm{Ac}\right) \mathrm{PO}_{4} @ 4 \mathrm{GdPO}_{4}$ shell@Au nanoparticles allow only $3 \%$ of ${ }^{213} \mathrm{Bi}$ ID to migrate to the kidney after 1 hour and $1.5 \%$ after 24 hours. In the core only $\mathrm{La}\left({ }^{225} \mathrm{Ac}\right) \mathrm{PO}_{4}$ system, $10 \%$ of the ${ }^{213} \mathrm{Bi}$ ID relocated to the kidney after 1 hour and 5\% after 24 hours.

While the $\left\{\mathrm{Gd}_{0.5} \mathrm{La}_{0.5}\right\} \mathrm{PO}_{4} @ 4 \mathrm{GdPO}_{4}$ shell@Au-PEG-mAb 201b nanoparticles clear rapidly from the lungs into the liver and spleen, previous strategies used to reduce reticuloendothelial functioning such as treatment with clodronate liposomes could be applied to mitigate the rapid clearance. ${ }^{104,105,106}$ Uptake at various time points as a result of injecting mice with clodronate liposomes prior to injection of $\left\{\mathrm{La}_{0.5} \mathrm{Gd}_{0.5}\right\}\left({ }^{225} \mathrm{Ac}\right) \mathrm{PO}_{4} @ 4 \mathrm{GdPO}_{4}$ shell@Au-mAb-201b or $\left\{\mathrm{La}_{0.55} \mathrm{Gd}_{0.75}\right\}\left({ }^{225} \mathrm{Ac}\right) \mathrm{PO}_{4} @ 4 \mathrm{LaPO}_{4}$ shell@Au-mAb-201b nanoparticles is shown in Figures 3.5.7 A-D.

Using clodronate liposomes drastically improves target tissue uptake at each time point. The liposomes selectively deplete macrophages, which are responsible for clearing large particulates such as nanoparticles from the bloodstream. At 1 hour, clodronate treated mice exhibit $46.7 \pm 1.8 \% \mathrm{ID}(327 \pm 36 \% \mathrm{ID} / \mathrm{g})$ lung uptake compared with $30.2 \pm 3.6 \%$ ID $(204 \pm 25 \%$ ID/g) in saline treated mice. Proportions are similar at the 24 hour and 48 hour time points for $\left\{G_{0.5} \mathrm{La}_{0.5}\right\}\left({ }^{225} \mathrm{Ac}\right) \mathrm{PO}_{4} @ 4 \mathrm{GdPO}_{4}$ shell@Au nanoparticles. The difference in uptake between mice treated with clodronate and 


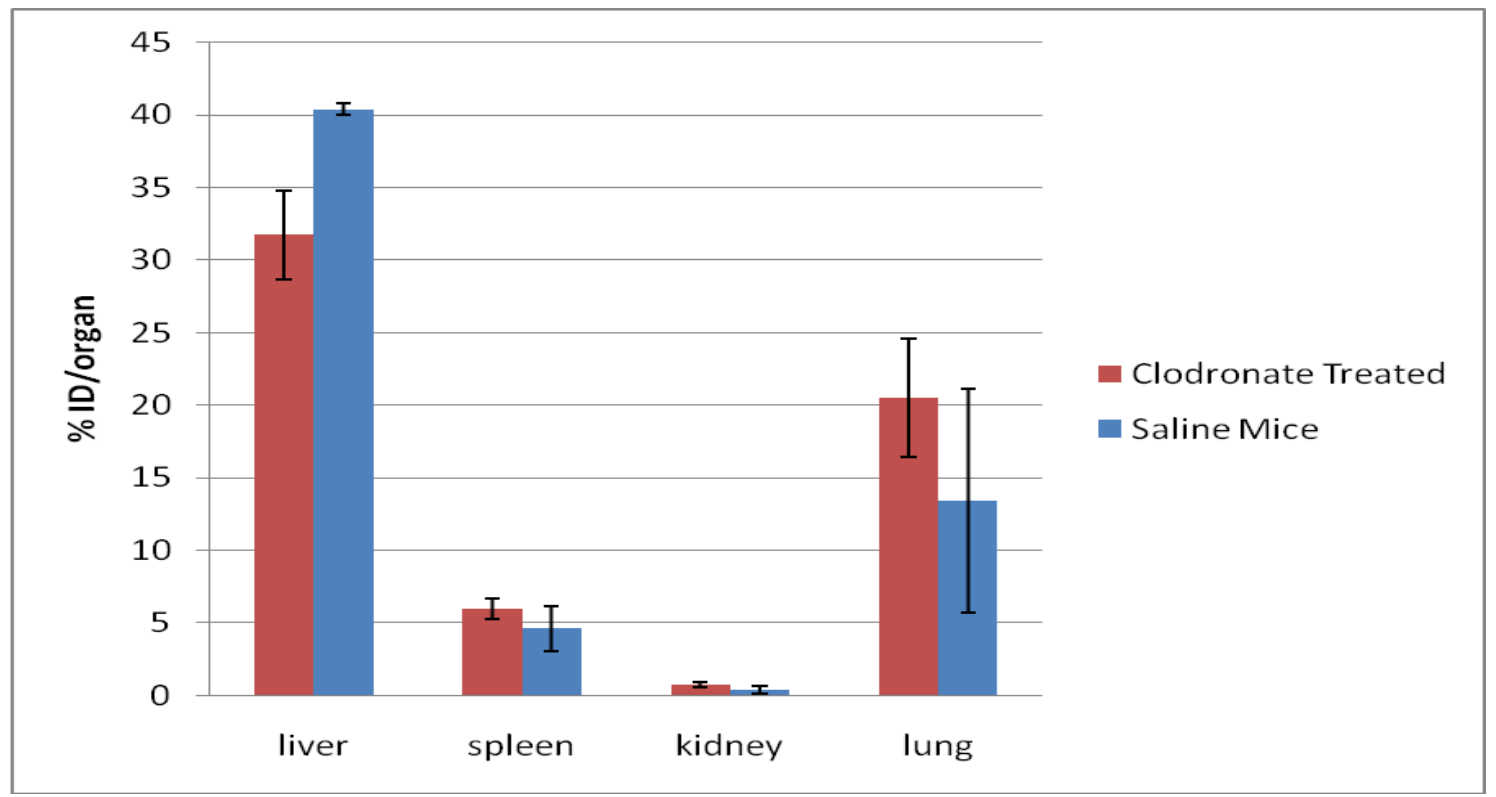

Figure 3.5.7 A: Nanoparticle uptake in major organs after one hour in mice injected with clodronate liposomes or saline followed by the $\left\{\mathrm{Gd}_{0.75} \mathrm{La}_{0.25}\right\}\left({ }^{225} \mathrm{Ac}\right) \mathrm{PO}_{4} @ 4 \mathrm{LaPO}_{4}$ shell@Au-201b system.

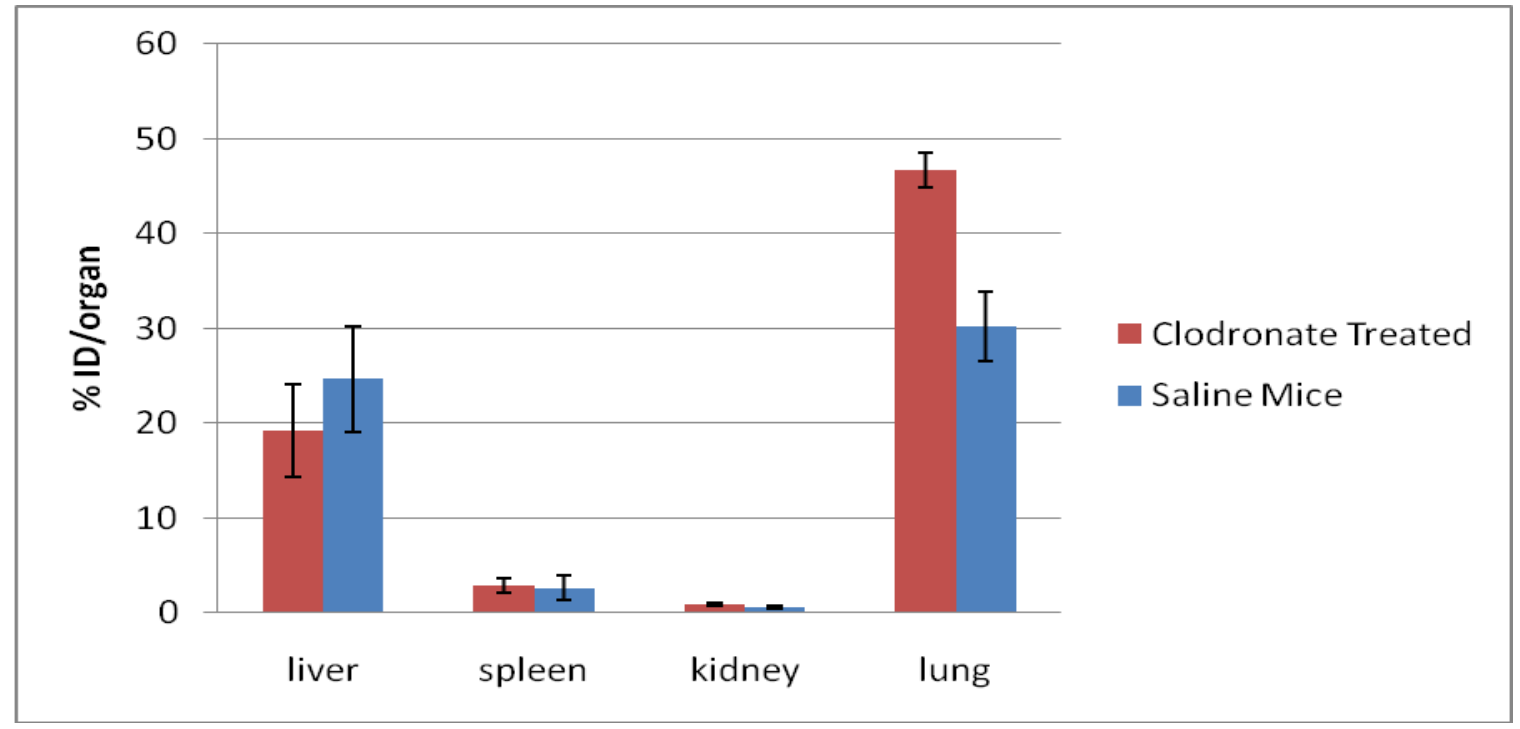

Figure 3.5.7 B: Nanoparticle uptake in major organs after one hour in mice injected with clodronate liposomes or saline followed by the $\left\{\mathrm{Gd}_{0.75} \mathrm{La}_{0.25}\right\}\left({ }^{225} \mathrm{Ac}\right) \mathrm{PO}_{4} @ 4 \mathrm{LaPO}_{4}$ shell@Au-201b system. 


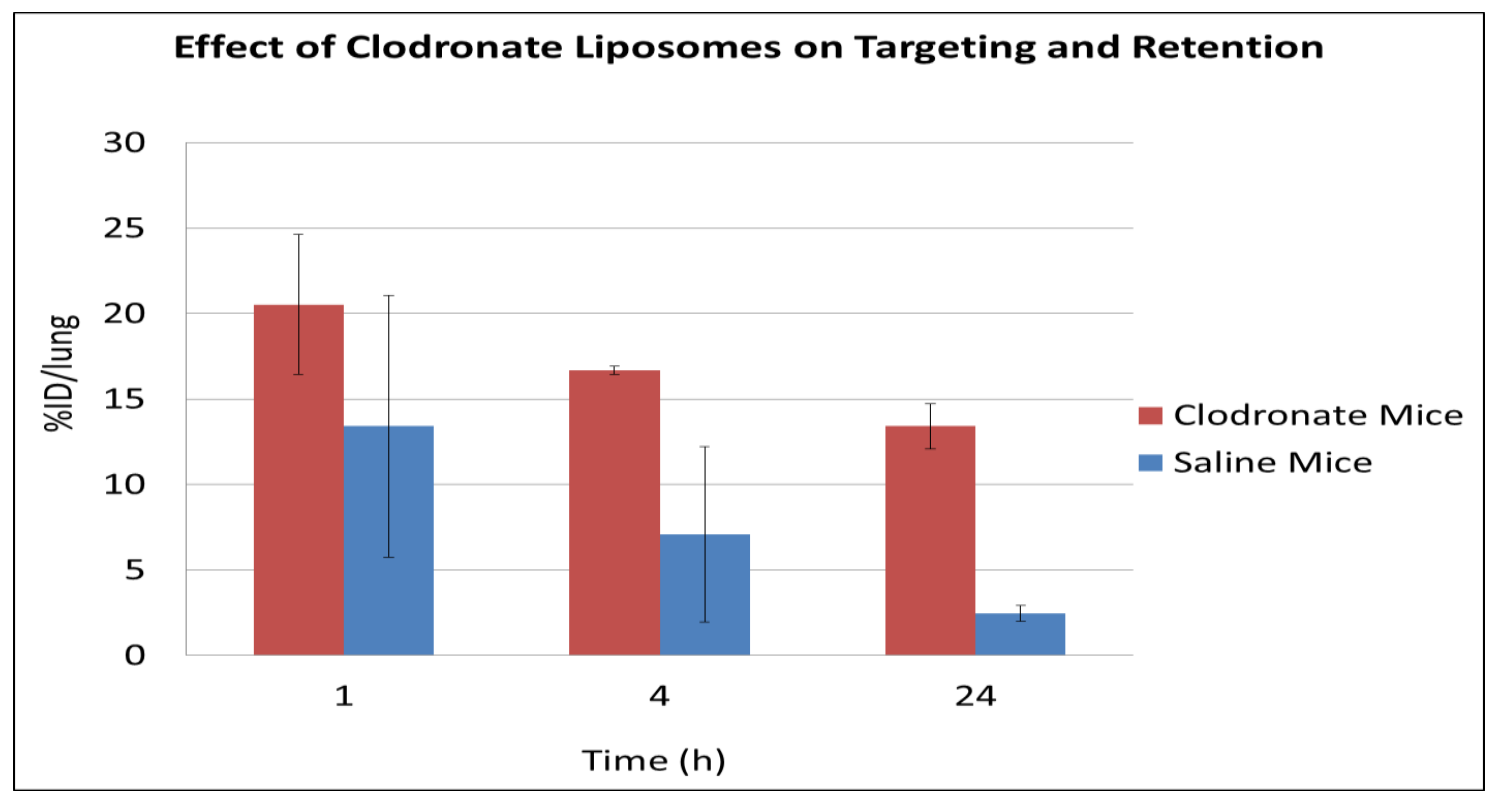

Figure 3.5.7 C: Change in lung uptake over time in mice treated with clodronate liposomes or saline followed by the $\left\{\mathrm{Gd}_{0.75} \mathrm{La}_{0.25}\right\}\left({ }^{225} \mathrm{Ac}\right) \mathrm{PO}_{4} @ 4 \mathrm{LaPO}_{4}$ shell@Au-201b system.

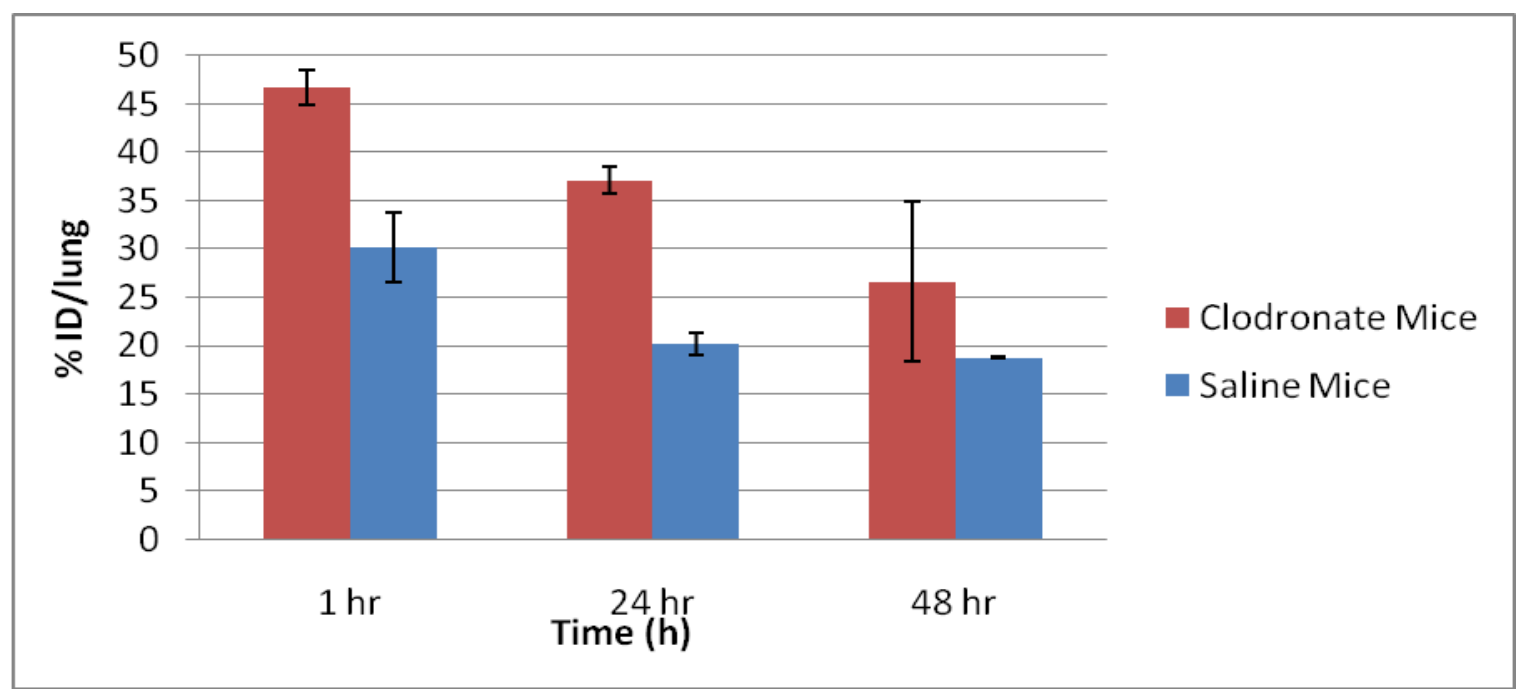

Figure 3.5.7 D: Change in lung uptake over time in mice injected with clodronate liposomes or saline followed by the $\left\{\mathrm{Gd}_{0.5} \mathrm{La}_{0.5}\right\}\left({ }^{225} \mathrm{Ac}\right) \mathrm{PO}_{4} @ 4 \mathrm{GdPO}_{4}$ shell@Au-201b system. 
those treated with saline appears to be almost exclusively due to clearance by the RES. Mice injected with clodronate and $\left\{\mathrm{Gd}_{0.75} \mathrm{La}_{0.25}\right\}\left({ }^{225} \mathrm{Ac}\right) \mathrm{PO}_{4} @ 4 \mathrm{LaPO}_{4}$ nanoparticles show 7.1\% higher uptake in the lung than their saline-injected counterparts. Combined increases in liver and spleen uptake by the saline-injected mice (7.3\%) mirror the decrease in lung uptake. The drastic difference in lung uptake after a time as short as 1 hour indicates how quickly the RES clears the nanoparticles from circulation without pre-injection of clodronate liposomes. Unlike many antibodies, mAb 201b quickly localizes to its target tissue due to the vascular availability of the thrombomodulin receptor. This short targeting time allows high uptake even with RES interference. After knocking out the RES, nanoparticle uptake and residence time in lung approaches that of the unconjugated antibody.

In addition to increasing lung uptake, clodronate liposomes also prolong residence time in lung tissue. After 24 hours, only $35 \%$ of the activity from $\left\{\mathrm{Gd}_{0.75} \mathrm{La}_{0.25}\right\}\left({ }^{225} \mathrm{Ac}\right) \mathrm{PO}_{4} @ 4 \mathrm{LaPO}_{4} @ \mathrm{Au}$ nanoparticles clears from the lung in the clodronate treated mice compared with $81.3 \%$ in the saline treated mice over the same time period. $\left\{\mathrm{Gd}_{0.5} \mathrm{La}_{0.5}\right\}\left({ }^{225} \mathrm{Ac}\right) \mathrm{PO}_{4} @ 4 \mathrm{GdPO}_{4}$ shell@Au nanoparticles also show prolonged nanoparticle residence time in lung tissue. After 24 hours, $21.6 \%$ of the initial uptake in the lung clears in the clodronate treated mice while $33.1 \%$ of the initial activity clears in the non-clodronate treated mice.

The nanoparticle-mAb $201 \mathrm{~b}$ conjugates show high retention of ${ }^{213} \mathrm{Bi}$ in the target lung tissue. After 1 hour, $70.4 \pm 0.8 \%$ of the ${ }^{213} \mathrm{Bi}$ remains in the lung. This number 108 
increases to $91.4 \pm 0.7 \%$ after 24 hours. Electron microscopy of non-radioactive $\left.\left\{\mathrm{Gd}_{0.5} \mathrm{La}_{0.5}\right\} \mathrm{C}^{225} \mathrm{Ac}\right) \mathrm{PO}_{4} @ 4 \mathrm{GdPO}_{4}$ shell@Au-mAb 201b conjugates shows the conjugates both lining the vascular space connected to lung epithelium, and internalized into the lung epithelial cells. Figure 3.5.4 further shows NPs during the process of endocytosis into a lung epithelial cell. Retention of daughter radionuclides by the nanoparticles themselves should theoretically remain constant and can be measured as previously described. ${ }^{48}$ As the nanoparticles are internalized in vivo, the cell itself augments the inherent capability of the nanoparticles to contain daughter radionuclides, increasing the effective daughter sequestration at the target site.

Therapy experiments using the EMT-6 model system show multiple small lung colonies. Group 1 (PBS) averaged $77.6 \pm 34.5$ colonies per tissue section area. Group 2 (nanoparticles with competition) showed fewer colonies ( $57 \pm 22.3$ colonies per slide). Finally, group 3 (targeted nanoparticles without competition) had drastically fewer colonies with only $20.8 \pm 7.5$ colonies per slide. Images of a representative section of tissue from each treatment group are shown in Figure 3.7.6. Additionally, Figure 3.7.7 shows a magnified version of the PBS treatment group with labels for tumor colonies and artifactual staining of macrophages/type II alveolar cells. 
PBS

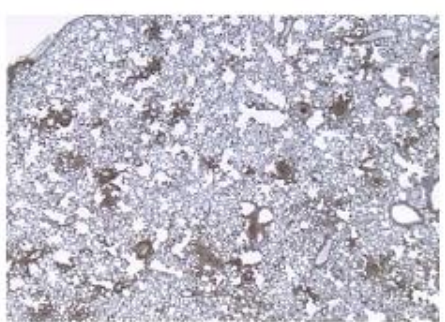

Ac-225 NPs with competition

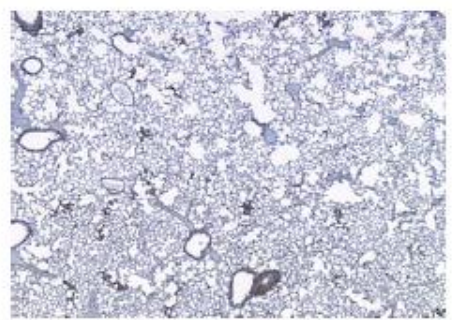

Targeted NPs

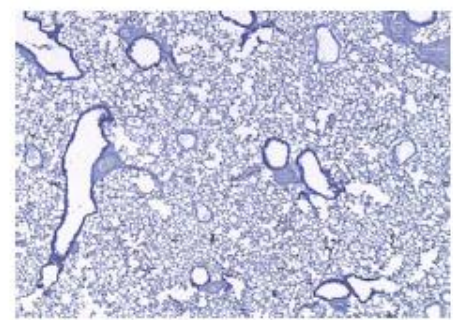

Figure 3.5.8: Stained slides showing EMT-6 tumor colonies present in lung tissue of mice treated with PBS, $\left\{\mathrm{Gd}_{0.5} \mathrm{La}_{0.5}\right\}\left({ }^{225} \mathrm{Ac}\right) \mathrm{PO}_{4} @ \mathrm{GdPO}_{4} @ \mathrm{Au}$ nanoparticles with competition from mAb 201b, or those same particles without competition.

\section{Tumor}

\section{Macrophage or type II}

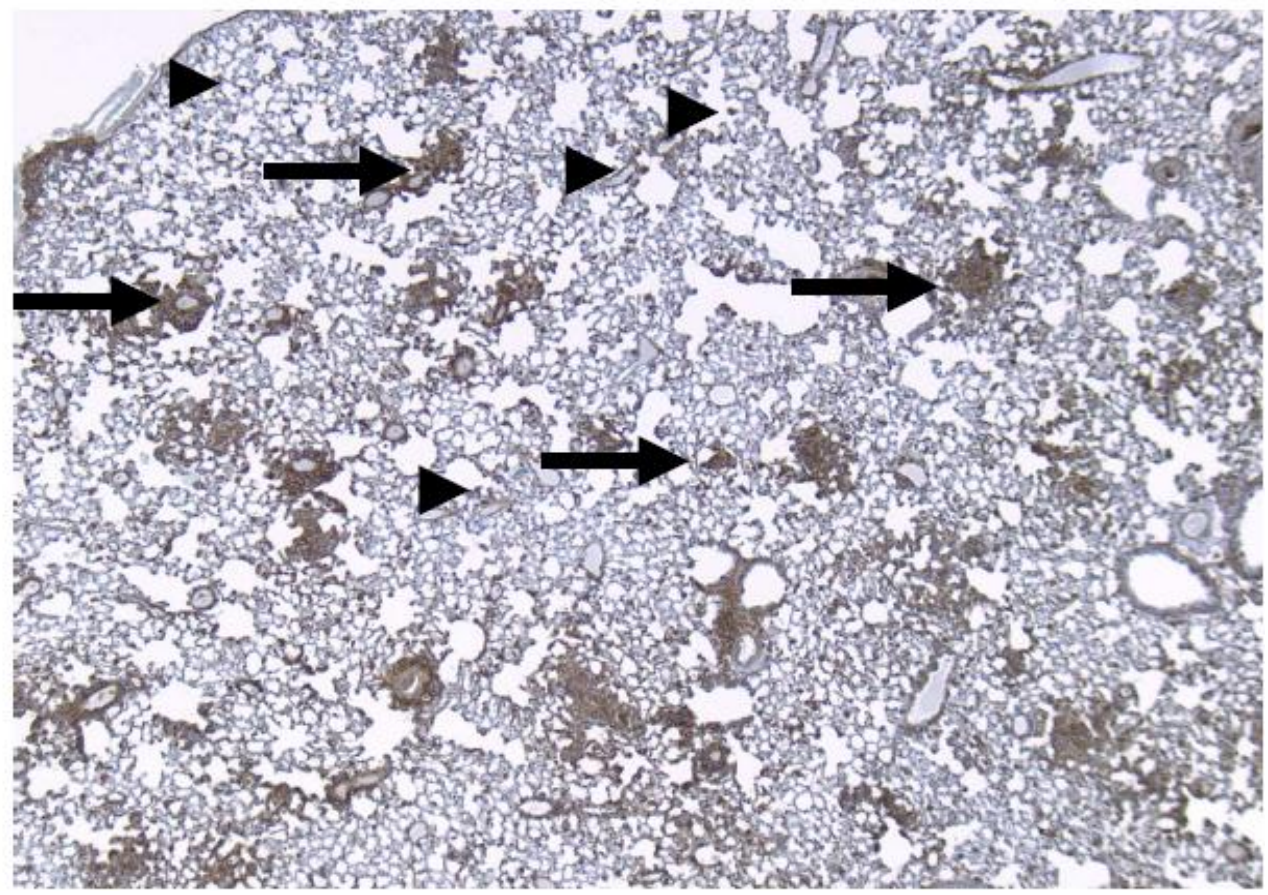

Figure 3.5.9: A stained slide from the PBS treatment group labeling lung tumor colonies as well as artifactual staining of macrophages and type II alveolar cells. 
The EMT-6 therapy experiment shows promising results for future work with more specific antibody targeting. Despite targeting the organ rather than the tumor cells, the treatment group shows clear, statistically significant $(p=0.019)$ decreases in tumor colony growth relative to both PBS and competition from free antibody $(p=$ 0.020). Competition with non-radioactive mAb $201 \mathrm{~b}$ decreases lung uptake by more than a factor of 17 . However, the lung in the competition group still receives $\sim 1.0 \mathrm{kBq}$ of ${ }^{225} \mathrm{Ac}$ after one hour. This small amount of ${ }^{225} \mathrm{Ac}$ is insufficient to produce a significant $(p=0.300)$ therapeutic effect relative to PBS controls. Higher uptake in the lung in the treatment group (17.3 kBq after one hour) produces the larger therapeutic effect. No abnormal side effects were observed during the 1 week observation period. TEM images from the mice confirm no inflammation in tissues of the kidney as a result of radiation or nanoparticle exposure. Previous experiments with ${ }^{225}$ Ac and EMT-6 tumors led to pulmonary fibrosis and renal failure from failure to retain ${ }^{213} \mathrm{Bi}$ daughters in the target tissue. ${ }^{107}$ Future therapeutic doses could therefore likely be increased without inducing fatal side effects.

\subsubsection{Brain Targeting with Anti-Synaptophysin and Anti-GFAP}

Utilizing targeted, short range therapeutics based on $\alpha$ particles could revolutionize treatment of tumors in vital organs such as the brain. Due to the vital importance of the brain in regulating bodily function, a number of evolutionary protections have developed to limit the flow of non-essential material into the tissue. While the series of tight junctions and barrier cells that constitute the blood-brain 
barrier protect brain tissue from pathogens, they also frustrate attempts to deliver therapeutics to the brain. Difficulty associated with side effects of neurosurgery often precludes surgical resection of brain tumors depending on the location of the neoplasm. Nanoparticles of approximately $20 \mathrm{~nm}$ metallic diameter or smaller show the ability to traverse the blood-brain barrier, sparking interest in nanoparticle-based therapies for central nervous system (CNS) disorders such as Alzheimer's disease. ${ }^{108,109}$

In vitro, nanoparticles bound to antibodies relevant to CNS tissue retain their biological activity. Murine brain tissue sections incubated with anti-synaptophysin labeled $\left\{\mathrm{Gd}_{0.5} \mathrm{La}_{0.5}\right\} \mathrm{PO}_{4} @ 4 \mathrm{GdPO}_{4}$ shell@Au nanoparticles show binding to the basal ganglia and synapses. Surrounding white matter does not show staining, indicating that the antibody retains its biological activity and specificity after binding to the nanoparticle. The staining process involves a number of steps. First, the nanoparticleprimary antibody conjugates bind their antigen receptors in the brain. Next, a secondary antibody contains a peroxidase enzyme and binds to the primary antibody in the tissue section. In peroxide, DAB is converted into a brown, insoluble product and gives a brown stain. Figure 3.5 .10 shows the immunohistochemistry of antisynaptophysin labeled nanoparticles. 


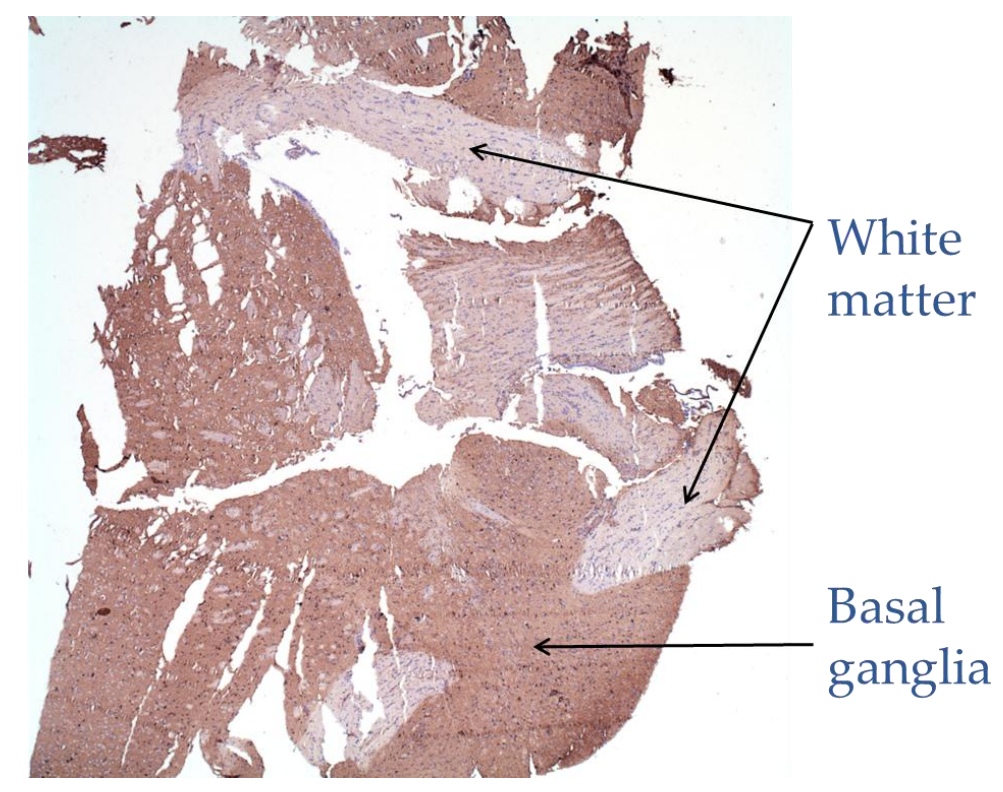

Figure 3.5.10: Immunohistochemistry of brain tissue with anti-synaptophysin labeled nanoparticles. Anti-synaptophysin labeled nanoparticles maintain biological activity and specificity after binding to the nanoparticles. Basal ganglia cells with nanoparticles bound are stained by the DAB color agent while adjacent white matter is not.

Similarly, anti-GFAP labeled nanoparticles retain their affinity and specificity for receptors after binding to the nanoparticle surface. Figure 3.5 .11 shows immunohistochemistry in normal murine brain. Astrocyte cells show staining by the DAB color agent, while surrounding brain tissue does not. Blood vessels in the brain exhibit artifactual staining as well. 


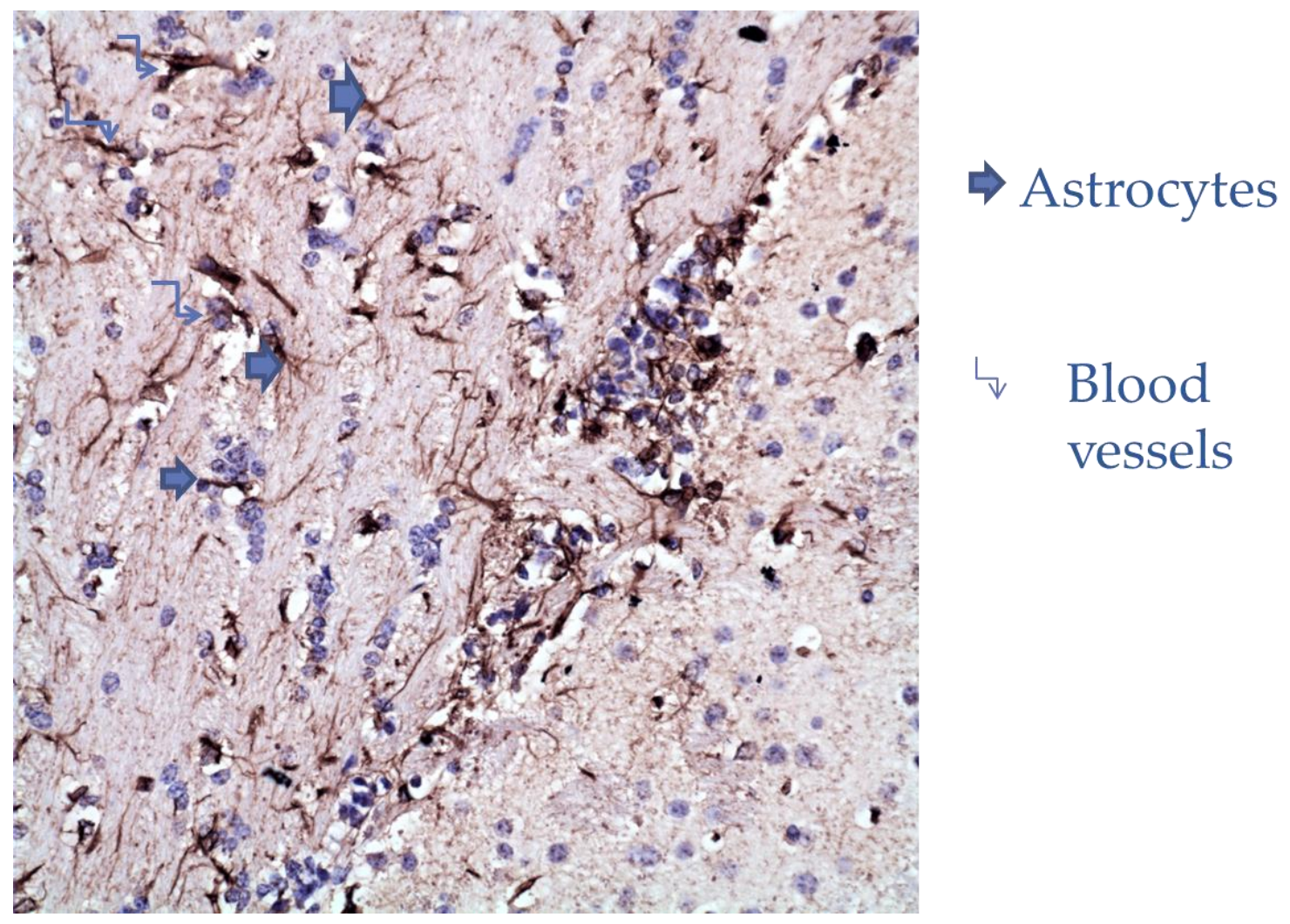

Figure 3.5.11: Immunohistochemistry of brain tissue with anti-GFAP labeled nanoparticles. Anti-GFAP labeled nanoparticles also retain their affinity and specificity for biological targets after binding to the nanoparticle surface. Brown areas indicated by large arrows illustrate astrocytes while small arrows illustrate artifactual staining of blood vessels.

For in vivo brain receptor experiments, mice were cannulated in the left femoral or axillary vein via partial surgical dissection. The surgical area was first shaved, then dissected using auto-cauterizers to minimize blood loss. This technique ensures that all of the injected dose reaches the bloodstream for consistent, reliable delivery. Utilizing a 
catheter also allows for multiple injections using a single point. Figure 3.5.12 shows the catheter inserted into a mouse femoral vein.

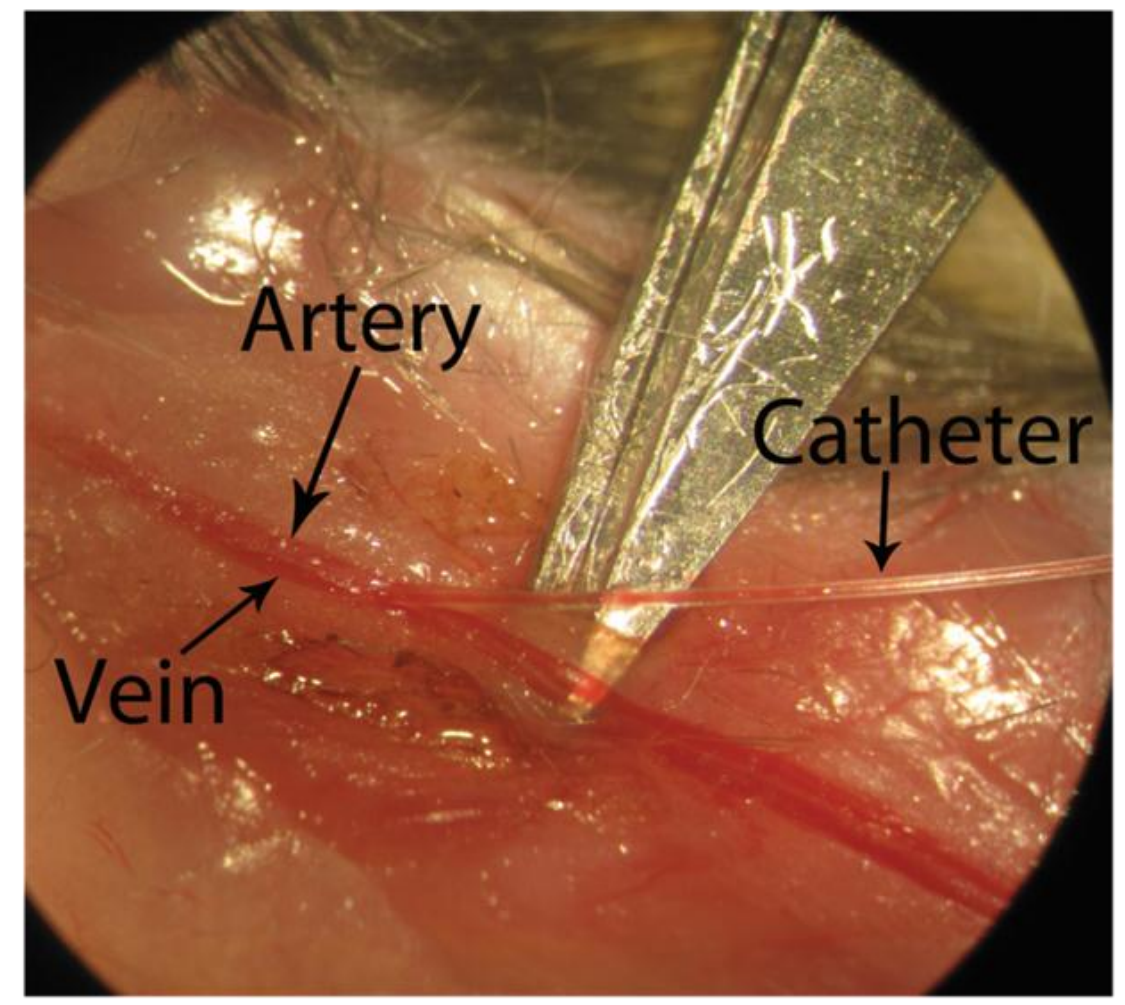

Figure 3.5.12: A catheter inserted into the left femoral vein aids injection reproducibility and allows for multiple injections at a single site.

Biodistribution experiments performed with NAA indicate that nanoparticle-mAb conjugates exhibit uptake in the brain. Both anti-synaptophysin and anti-GFAP antibodies show uptake of $0.1 \%$ ID. In each case, the measurement strained MURR's detection limit of NAA for gold of $0.1 \mathrm{ng}$. However, the low level of detection is 
consistent across six mice, lending credibility to the measurement. Unlabeled nanoparticles were not detected in the brain tissue in any of the mice examined.

In order to confirm that the nanoparticle-mAb conjugates are specifically targeting their receptors in the brain and not merely present in the blood vasculature, brain tissue sections were fixed and examined by TEM. Figures 3.5.13 and 3.5.14 show representative images of anti-synaptophysin labeled nanoparticles present at CNS synapses and anti-GFAP labeled nanoparticles present by astrocytes.

Mannitol creates a hyperosmotic environment in CNS arteries, causing water loss in the CNS epithelial cells and stretching the tight junctions. ${ }^{110}$ TEM images show that the tight junctions of the blood brain barrier have not been disrupted, despite injection of mannitol for that purpose. Because the tight junctions remain largely intact, another mechanism must be responsible for the presence of large nanoparticles in the brain. As in the lung, the nanoparticles most likely extravasate out of the vasculature through an endocytotic mechanism. Vascular receptors similar to synaptophysin and GFAP mediate the endocytosis, importing only those materials necessary to CNS function. Nanoparticles bound to the anti-synaptophysin and anti-GFAP antibodies piggyback on this interaction, and are also endocytosed. 


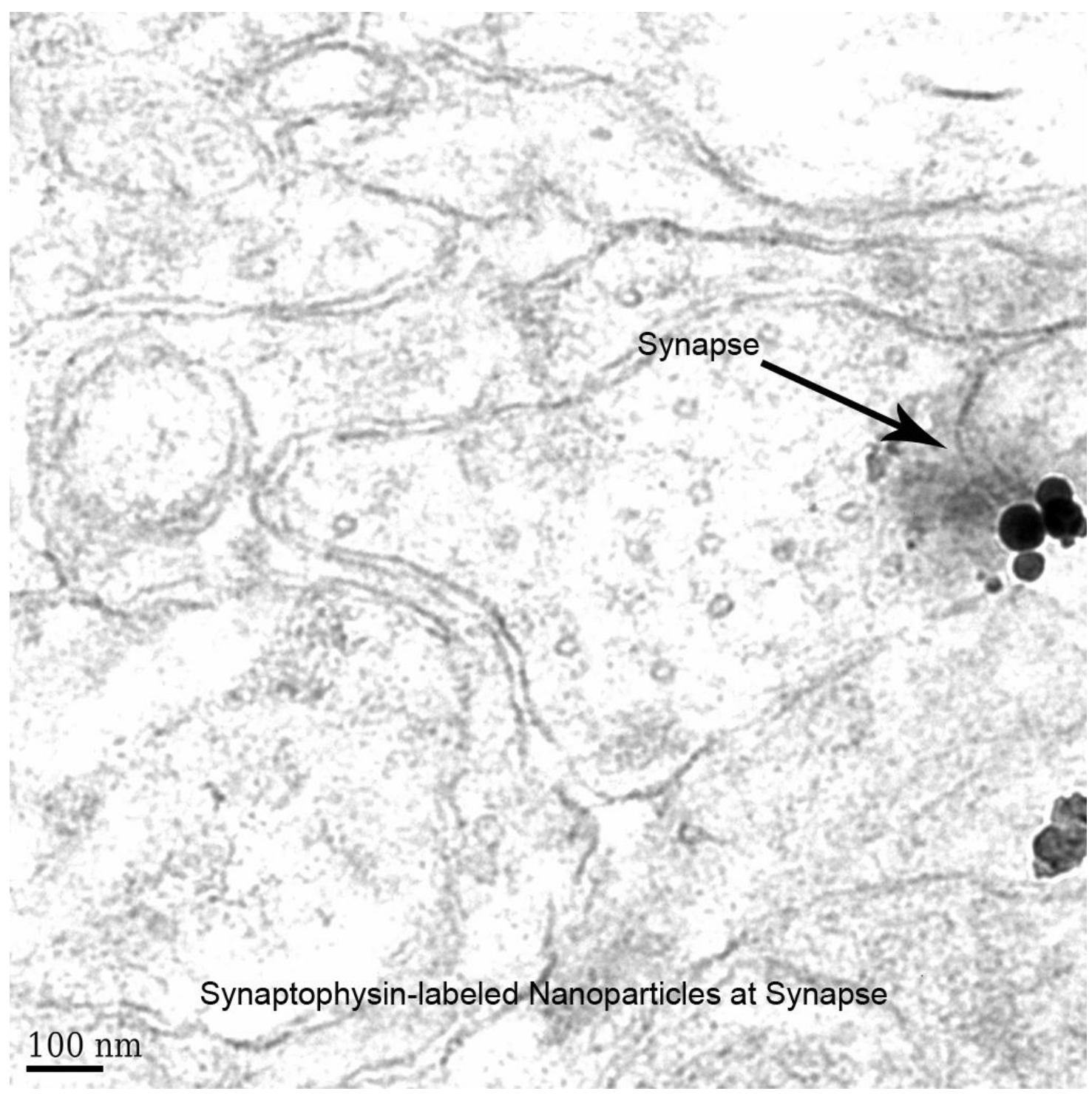

Figure 3.5.13: $\left\{\mathrm{Gd}_{0.5} \mathrm{La}_{0.5}\right\} \mathrm{PO}_{4} @ 4 \mathrm{GdPO}_{4}$ shell@Au nanoparticles labeled with antisynaptophysin bind receptors in CNS synapses. 


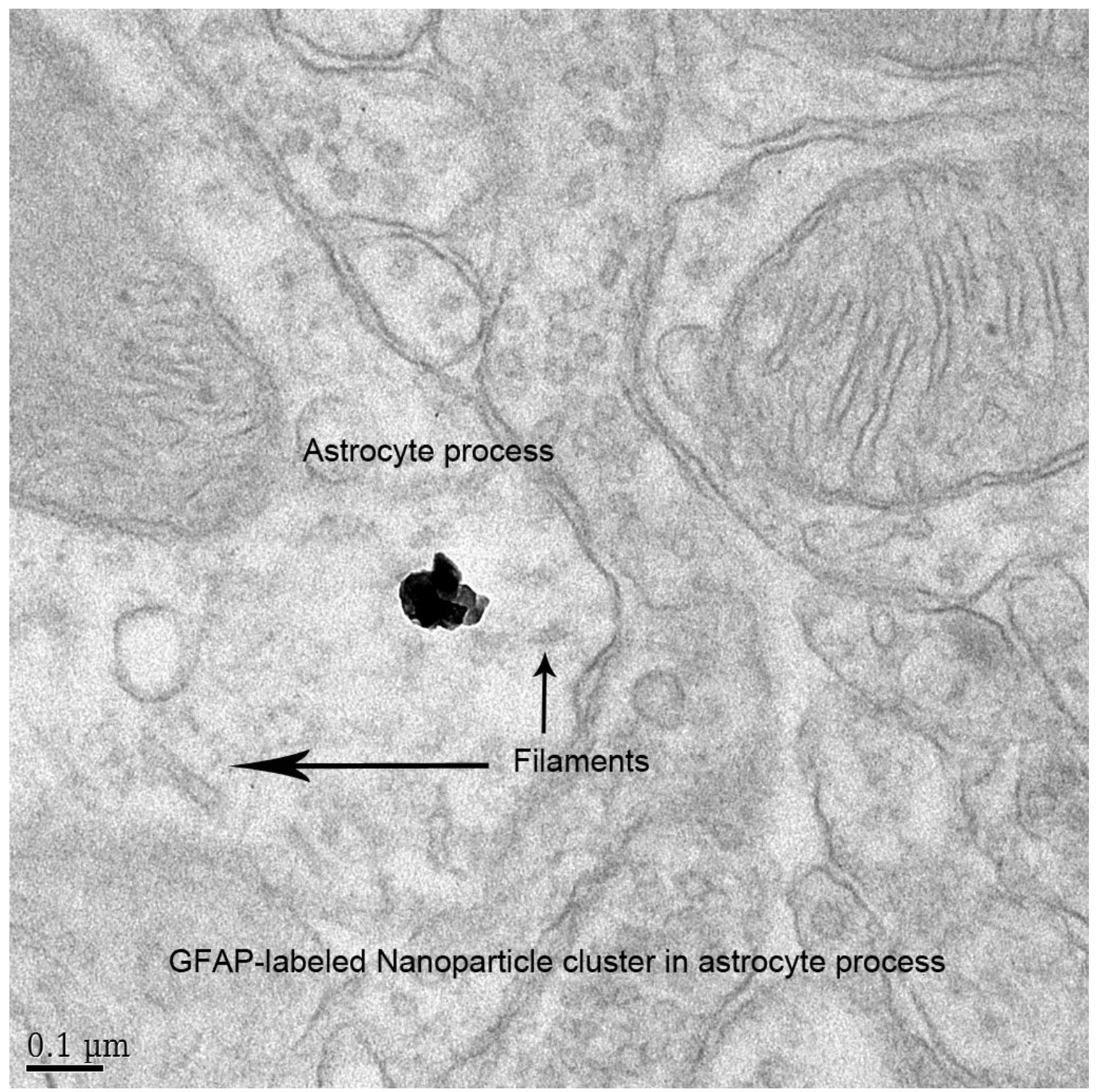

Figure 3.5.14: $\left\{\mathrm{Gd}_{0.5} \mathrm{La}_{0.5}\right\} \mathrm{PO}_{4} @ 4 \mathrm{GdPO}_{4}$ shell@Au nanoparticles labeled with anti-GFAP bind receptors in astrocytes. 


\subsection{Summary of Findings}

A central problem facing in vivo $\alpha$ generators has been control of daughter radionuclides. Historically, this lack of retention has led to lethal dose to non-target organs, limiting the efficacy of these systems. The work presented in Chapter 3 elucidates a general solution to the problem of daughter retention through the use of layered nanoparticles. The most successful system consisting of $\left\{\mathrm{Gd}_{0.5} \mathrm{La}_{0.5}\right\} \mathrm{PO}_{4} @ 4$ $\mathrm{GdPO}_{4}$ shell@Au nanoparticles contained $90 \%$ of the first decay daughter, ${ }^{221} \mathrm{Fr}$, in vitro. In vivo results show retentions of $>90 \%$ of the third decay daughter, ${ }^{213} \mathrm{Bi}$, after 24 hours. Retention in vivo increases over time as a result of higher percentages of endocytosed nanoparticles. Finally, the nanoparticles can be linked to a variety of different targeting agents for treatment of a wide variety of neoplastic disease.

Given the success of the layered nanoparticle system in containing daughter

emissions from ${ }^{225} \mathrm{Ac}$, the general strategy should be extended to other $\alpha$-generator radionuclides such as ${ }^{227}$ Th or ${ }^{223}$ Ra. Diversifying to these other $\alpha$-emitters would allow clinical tailoring of $\alpha$ energies and daughter chemistry/half-lives to the specific disease. Preparing a variety of layered nanoparticle formulations which work with $\alpha$-generator radionuclides will decrease the time required for clinical translation once these radionuclides become available in large quantities. 


\section{Chapter 4:}

\section{FUTURE DIRECTIONS AND ONGOING WORK}

\subsection{Other Lanthanides as Radiotherapeutics}

\subsubsection{Nuclear and Chemical Properties of the Lanthanides}

While the nanoparticles examined in this work focused on utilizing lanthanum and gadolinium, a number of other lanthanides should co-crystallize into $\mathrm{LnPO}_{4}$ nanoparticles. Fortunately, a number of other lanthanides also possess favorable nuclear properties for $\beta^{-}$radiotherapy. A similar synthetic process and gold surface modification should work for a variety of different lanthanides. Moving across the period, the lanthanide contraction causes large disparities in ionic radii. Extremes such as $\mathrm{La}^{3+}(1.18 \AA)$ and $\mathrm{Lu}^{3+}(1.00 \AA)$ may not co-crystallize. ${ }^{97}$ However, mixed lanthanide particles may not be necessary if the lanthanide possesses a sufficient magnetic moment to be separated magnetically.

Many lanthanides are under consideration or currently in use for biomedical purposes including ${ }^{149} \mathrm{Pm}\left(\beta^{-}\right.$cancer therapy), ${ }^{111}{ }^{153} \mathrm{Sm}$ (Quadramet, $\beta^{-}$pain palliation in bone metastases), ${ }^{112} \mathrm{Eu}$ (fluorescence imaging probes), ${ }^{113}{ }^{159} \mathrm{Gd}$ (Auger electron cancer 
therapy), ${ }^{114}{ }^{161} \mathrm{~Tb}\left(\beta^{-}\right.$cancer therapy), ${ }^{115}{ }^{166} \mathrm{Dy} /{ }^{166} \mathrm{Ho}\left(\beta^{-}\right.$arthritis therapy $){ }^{116},{ }^{169} \mathrm{Er}\left(\beta^{-}\right.$ arthritis therapy), ${ }^{117}{ }^{175} \mathrm{Yb}$ ( $\beta^{-}$bone pain palliation), ${ }^{118}$ and ${ }^{177} \mathrm{Lu}\left(\beta^{-}\right.$cancer therapy). ${ }^{119}$ Only a few of these $\left({ }^{149} \mathrm{Pm},{ }^{166} \mathrm{Ho}\right.$, and $\left.{ }^{177} \mathrm{Lu}\right)$ are available as no carrier added radionuclides. For all of the other radionuclides, only a small percentage of bioconjugates will deliver radiation to the intended target. Incorporating the radionuclides into a nanoparticle would dramatically increase the amount of radioactivity bound to each targeting vector. Radionuclides that are available no carrier added could also use nanoparticle constructs to increase the delivered dose. In order to determine the feasibility of this approach, nanoparticles composed of $\mathrm{TbPO}_{4}, \mathrm{HoPO}_{4}$, and $\mathrm{LuPO}_{4}$ were synthesized.

\subsubsection{Synthesis and Characterization of $\mathrm{TbPO}_{4}, \mathrm{HoPO}_{4}$, and $\mathrm{LuPO}_{4}$ Nanoparticles}

Nanoparticles consisting of $\mathrm{TbPO}_{4}, \mathrm{HoPO}_{4}$, and $\mathrm{LuPO}_{4}$ were synthesized similarly to 3.2.3. Briefly, $1 \mathrm{~mL}$ of $0.1 \mathrm{M} \mathrm{Tb}\left(\mathrm{NO}_{3}\right)_{3} \cdot 5 \mathrm{H}_{2} \mathrm{O}, \mathrm{HoCl}_{3}$, or $\mathrm{LuCl}_{3} \cdot 6 \mathrm{H}_{2} \mathrm{O}$ was added to a 5 $\mathrm{mL}$-bottom vial with spin vane and $2 \mathrm{~mL}$ of $0.1 \mathrm{M} \mathrm{Na}$-TPP. Each vial was heated for 3 hours at $90^{\circ} \mathrm{C}$. Nanoparticles were transferred into scintillation vials and placed by a 0.6 T surface field NdFeB magnet to determine if they could be magnetically separated. $\mathrm{LuPO}_{4}$ nanoparticles were dialyzed against $18 \mathrm{M} \Omega$ water using a $10 \mathrm{kDa}$ MWCO regenerated cellulose membrane overnight, then collected and dried over low heat. Dried particles were crushed using a mortar and pestle, then examined using XRD as described in 3.2.9. 


\subsubsection{Results and Discussion}

$\mathrm{TbPO}_{4}, \mathrm{HoPO}_{4}$, and $\mathrm{LuPO}_{4}$ all formed nanoparticle solutions similar to previous $\mathrm{LnPO}_{4}$ combinations. $\mathrm{TbPO}_{4}$ and $\mathrm{HoPO}_{4}$ nanoparticles both showed strong preference towards the magnet and separated cleanly. $\mathrm{LuPO}_{4}$ nanoparticles on the other hand showed no preference for the magnet and settled uniformly on the bottom of the vial. Of the lanthanides, gadolinium is most often used as a magnetic agent, due to its 7 unpaired $\mathrm{f}$ electrons in its preferred $3+$ oxidation state. While terbium (6 unpaired electrons in the $3+$ oxidation state) and holmium ( 4 unpaired electrons in the $3+$ oxidation state) should exhibit a lower paramagnetic magnetic moment than gadolinium, it still appears sufficient to facilitate magnetic separation. In contrast, lutetium ( 0 unpaired electrons in the $3+$ oxidation state) shows no paramagnetic

moment. As a result, ${ }^{177}$ Lu would need to be paired with a more magnetic lanthanide such as gadolinium in order to separate the nanoparticles magnetically.

XRD studies indicate that $\mathrm{LuPO}_{4}$ crystallizes in the anhydrous monazite crystal phase, similar to that observed for $\mathrm{GdPO}_{4}$ and mixtures of $\mathrm{LaPO}_{4}$ and $\mathrm{GdPO}_{4}$. The XRD pattern is shown below in Figure 4.1.1. Rietvield refinement indicates that the grain size of these particles is $1.76 \mathrm{~nm}$, somewhat smaller than the other $\mathrm{LnPO}_{4}$ systems previously analyzed. 


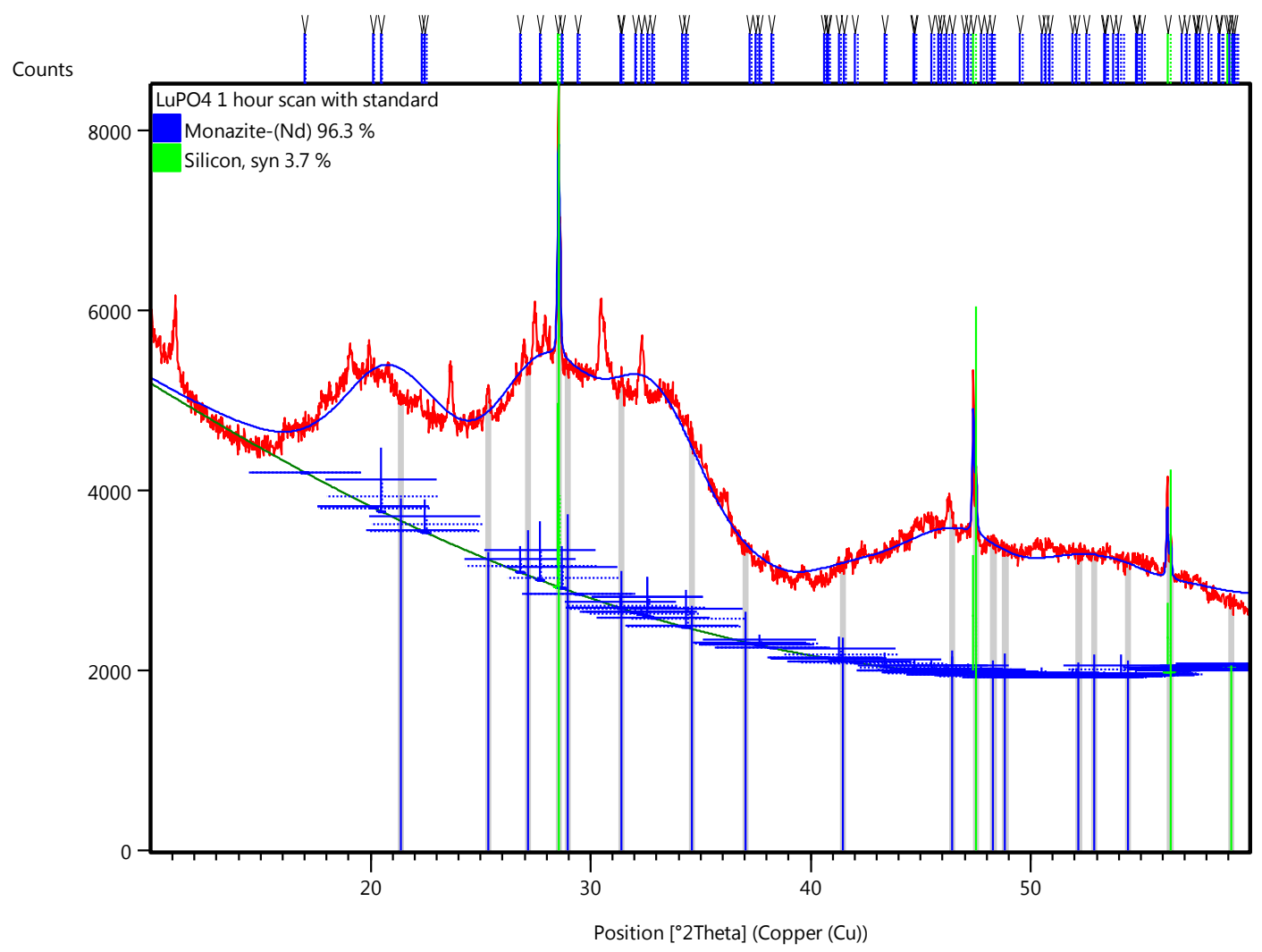

Figure 4.1.1: XRD pattern of $\mathrm{LuPO}_{4}$ indicates a monazite crystal structure with grain size of $1.76 \mathrm{~nm}$.

\subsubsection{Conclusions}

A number of lanthanides could easily work in the platform described in Chapter 3. Given the large quantity of ${ }^{177}$ Lu produced at MURR, investigation of this radionuclide makes particular sense at Missouri. XRD studies indicate that a nanoparticle similar in structure to previously studied constructs is formed under similar conditions. Ongoing experiments involving synthesis of a mixed $\left\{\mathrm{Gd}_{0.5}{ }^{177} \mathrm{Lu}_{0.5}\right\} \mathrm{PO}_{4}$ nanoparticle are in 
progress under the direction of fellow graduate student Nick Sobol. Gold coating of the particles should occur analogously and similarly facilitate attachment of biologically relevant targeting agents.

\subsection{Potential for ${ }^{227}$ Th or ${ }^{223}$ Ra generators}

\subsubsection{Impetus for ${ }^{227}$ Th or ${ }^{223}$ Ra generators}

As mentioned in Chapter $1,{ }^{227} \mathrm{Th}$ and ${ }^{223} \mathrm{Ra}$ are also being considered as potential in vivo $\alpha$ generator radionuclides. Further, the higher availability of ${ }^{227} \mathrm{Th}$ and ${ }^{223} \mathrm{Ra}$ avoid the research and clinical trial bottleneck found with ${ }^{225} \mathrm{Ac}$. While ${ }^{223} \mathrm{Ra}$ as Alpharadin inherently targets bone metastases, incorporation into a $\mathrm{LnPO}_{4}$ nanoparticle could broaden its clinical applicability. In order to test the ability of $\mathrm{LnPO}_{4}$ as a carrier for ${ }^{223} \mathrm{Ra}$, barium was added to a synthesis of $\left\{\mathrm{Gd}_{0.5} \mathrm{La}_{0.5}\right\} \mathrm{PO}_{4}$ nanoparticles as a surrogate for radium. Radium and barium share a common preferred oxidation state $(2+)$ and similar 8-coordinate ionic radii $(\mathrm{Ba}=1.56 \AA$, $\mathrm{Ra}=1.62 \AA){ }^{97}$

Similarly, ${ }^{227}$ Th should be investigated for its potential to dope into $\mathrm{LnPO}_{4}$ nanoparticles. Previous work indicates that the lanthanide $\mathrm{Ce}^{+4}$ cocrystallizes well with $\mathrm{La}^{+3}$ to form fluorescent rhabdophane nanocrystals. ${ }^{120}$ The $\mathrm{Ce}^{+4}$ ion should function well as a surrogate for ${ }^{227} \mathrm{Th}$. Both elements easily access the $4+$ oxidation state and have similar 8-coordinate ionic radii $\left(\mathrm{Ce}^{4+}=1.11 \AA \mathrm{Th}^{4+}=1.19 \AA\right) .{ }^{97}$ 


\subsubsection{Barium nanoparticles experimental}

A solution of $500 \mu \mathrm{L}$ of $0.1 \mathrm{M} \mathrm{LaCl}_{3} \cdot 7 \mathrm{H}_{2} \mathrm{O}, 500 \mu \mathrm{L}$ of $0.1 \mathrm{M} \mathrm{GdCl}_{3} \cdot 6 \mathrm{H}_{2} \mathrm{O}, 50 \mu \mathrm{L}$ of $0.1 \mathrm{M} \mathrm{BaCl}_{2}$, and $2 \mathrm{~mL}$ of $0.1 \mathrm{M} \mathrm{Na}$-TPP were mixed in a $5 \mathrm{~mL}$-bottom vial with spin vane. The solution was heated for 3 hours at $90^{\circ} \mathrm{C}$.

\subsubsection{Ba nanoparticles results and discussion}

Nanoparticles with Ba dopants formed large aggregates that would not disperse in solution. It is likely that $\mathrm{Ba}_{3}\left(\mathrm{PO}_{4}\right)_{2}$ clumps formed rather than doping into the $\mathrm{LnPO}_{4}$ nanoparticles. The oxidation state and size mismatch between barium and the lanthanides likely preclude co-crystallization. While it may be possible to dope ${ }^{223} \mathrm{Ra}$ into $\mathrm{LnPO}_{4}$ nanoparticles on the tracer level, other barium containing nanoparticles such as $\mathrm{BaTiO}_{3},{ }^{121} \mathrm{BaSO}_{4},{ }^{122}$ or $\mathrm{BaFe}_{12} \mathrm{O}_{19}{ }^{123}$ should be more promising.

\subsubsection{Conclusion}

Oxidation state and size appear to be the defining factors that influence cocrystallization. While ${ }^{223} \mathrm{Ra}$ may not work well with $\mathrm{LnPO}_{4},{ }^{227} \mathrm{Th}$ could likely cocrystallize into $\mathrm{LnPO}_{4}$ nanoparticles containing a significant fraction of $\mathrm{Ce}^{4+}$ ions. Successful doping of $\mathrm{Ce}^{4+}$ ions into $\mathrm{LnPO}_{4}$ nanoparticles has already been demonstrated. ${ }^{124}$ Additionally, thorium doping itself could be done with natural thorium rather than the less common ${ }^{227}$ Th in initial experiments. 


\subsection{Future Clinical Applications}

While proof of principle targeting with mAb 201b showed high, receptor specific uptake, the thrombomodulin target has little clinical relevance to cancer therapy. Additional antibodies which target receptors overexpressed on tumors should be utilized to determine clinical applicability of the nanoparticle system. As detailed in Sections 1.3 and 1.4 , the short range of $\alpha$ emission and large size of nanoparticles limit potential application of the nanoparticle system to small metastases with vascular targets, circulating neoplasms, peritoneal tumors, and intratumoral injections. Utilizing vascular targeting agents such as $\mathrm{F} 3^{40}$ removes the necessity for nanoparticles to extravasate out of the vasculature.

Another potentially viable strategy to capitalize on the advantages of nanoparticle systems involves targeting cancers of the circulatory system. In particular, a wide variety of leukemias and lymphomas should be treatable with appropriate targeting agents. Targets such as CD20 (the same target used in the clinically approved BEXXAR radiopharmaceutical) ${ }^{125}$ and $C D 45^{126}$ are overexpressed on a number of bloodborne cancers. Antibodies that target these receptors, such as $1 F 5^{127}(C D 20)$ and $B C 8^{128}$ (CD45), are currently being investigated in cell pellet and mouse studies with the described $\mathrm{LnPO}_{4}$ nanoparticle system in collaboration with the Fred Hutchinson Cancer Research Center in Seattle, Washington. 
Additionally, tumors of the peritoneum tend to form thin films. ${ }^{129}$ Cancers originating in the peritoneal cavity (ovarian, liver, pancreatic, colorectal, gastric, and peritoneal mesothelioma) have high rates of locoregional metastasis to other locations within the peritoneal cavity. ${ }^{130}$ Administration of nanoparticles directly into the peritoneal cavity would also bypass the need for extravasation out of the vasculature. Additionally, $\alpha$ therapy is ideal for treating the thin cancerous films and metastases found in peritoneal cancers. Panitumumab, an antibody against EGFR conjugated to the particles in 3.4, may be an effective therapy for these peritoneal cancers.

Biodistribution studies are currently underway in collaboration with the National Cancer Institute.

Finally, $\alpha$ therapeutic nanoparticles can be injected directly into tumors as a brachytherapy analog. The solution of nanoparticles may perfuse throughout the target tissue, providing better coverage and lower whole body dose than current seed based therapies. More work is needed to determine the degree of perfusion in these therapies as well as the tissue retention of the nanoparticles over time. 
Appendix 1: Total biodistribution of starch-bombesin gold nanoparticles

\begin{tabular}{|c|c|c|c|c|}
\hline \multirow{2}{*}{$\begin{array}{c}\text { Compound: } \\
\text { Date: }\end{array}$} & \multicolumn{3}{|c|}{${ }^{198}$ Au-NP (Starch) (I.P.) } & \multirow[t]{2}{*}{$130 \mathrm{kBq} / 100 \mu \mathrm{L}$} \\
\hline & $8 / 18 / 2010$ & Tumor: & PC-3 & \\
\hline Time : & 2 Hour & Strain: & SCIDs & 5 mice \\
\hline Blocker: & Saline (I.V.) & Dose Cts. & 503838 & \\
\hline Organ & Avg \%ID & STDEV & Avg\%ID/g & STDEV \\
\hline Blood & 9.83 & 5.27 & 7.26 & 3.20 \\
\hline Heart & 0.06 & 0.06 & 0.37 & 0.46 \\
\hline Lung & 0.12 & 0.07 & 0.46 & 0.41 \\
\hline Liver & 5.34 & 2.33 & 4.55 & 2.21 \\
\hline Spleen & 0.85 & 0.36 & 9.32 & 5.72 \\
\hline Stomach & 3.87 & 1.26 & 13.17 & 11.59 \\
\hline L Int & 5.72 & 2.88 & 5.82 & 3.36 \\
\hline Sm Int & 12.93 & 9.61 & 10.64 & 8.63 \\
\hline Kidney & 0.42 & 0.33 & 1.28 & 1.21 \\
\hline Urine & 0.01 & 0.01 & & \\
\hline Abdominal Fat & 31.86 & 28.55 & 127.26 & 115.98 \\
\hline Muscle & 0.01 & 0.01 & 0.03 & 0.03 \\
\hline Bone & 0.00 & 0.00 & 0.05 & 0.05 \\
\hline Bladder & 0.23 & 0.14 & 7.81 & 6.84 \\
\hline Brain & 0.00 & 0.00 & 0.00 & 0.00 \\
\hline Pancreas & 0.39 & 0.43 & 11.05 & 10.22 \\
\hline Carcass & 35.39 & 19.32 & 13.67 & 1.05 \\
\hline Tumor A & 0.02 & 0.02 & 0.06 & 0.06 \\
\hline
\end{tabular}




\begin{tabular}{|c|c|c|c|c|}
\hline \multirow{2}{*}{$\begin{array}{c}\text { Compound: } \\
\text { Date: }\end{array}$} & \multicolumn{3}{|c|}{${ }^{198} \mathrm{Au}-\mathrm{NP}$ (Starch) (I.P.) } & \multirow[t]{2}{*}{$130 \mathrm{kBq} / 100 \mu \mathrm{L}$} \\
\hline & $8 / 18 / 2010$ & Tumor: & PC-3 & \\
\hline Time : & 2 Hour & Strain: & SCIDs & 5 mice \\
\hline Blocker: & \multicolumn{2}{|c|}{ Cold BBN (I.V.) } & Dose Cts. & 503838.2 \\
\hline Organ & Avg \%ID & STDEV & Avg\%ID/g & STDEV \\
\hline Blood & 12.81 & 10.87 & 9.44 & 6.95 \\
\hline Heart & 0.07 & 0.11 & 0.61 & 0.97 \\
\hline Lung & 0.58 & 0.33 & 2.57 & 1.71 \\
\hline Liver & 7.70 & 2.06 & 6.62 & 1.48 \\
\hline Spleen & 0.78 & 0.45 & 8.43 & 7.22 \\
\hline Stomach & 6.48 & 3.20 & 9.23 & 4.26 \\
\hline L Int & 5.35 & 1.13 & 5.49 & 1.22 \\
\hline Sm Int & 10.11 & 7.29 & 6.67 & 3.72 \\
\hline Kidney & 0.84 & 0.38 & 2.59 & 1.15 \\
\hline Urine & 0.01 & 0.01 & & \\
\hline Abdominal Fat & 20.19 & 16.23 & 89.28 & 96.06 \\
\hline Muscle & 0.12 & 0.22 & 0.70 & 1.43 \\
\hline Bone & 0.01 & 0.01 & 0.18 & 0.36 \\
\hline Bladder & 0.23 & 0.13 & 6.75 & 5.80 \\
\hline Brain & 0.00 & 0.00 & 0.01 & 0.01 \\
\hline Pancreas & 0.92 & 0.31 & 22.01 & 9.07 \\
\hline Carcass & 42.13 & 6.84 & 11.04 & 0.44 \\
\hline Tumor A & 0.05 & 0.04 & 0.17 & 0.13 \\
\hline
\end{tabular}




\begin{tabular}{|c|c|c|c|c|}
\hline \multirow{2}{*}{$\begin{array}{c}\text { Compound: } \\
\text { Date: }\end{array}$} & \multicolumn{2}{|c|}{${ }^{198}$ Au-BBN-NP (I.P.) } & \multirow[b]{2}{*}{ PC-3 } & \multirow[t]{2}{*}{$130 \mathrm{kBq} / 100 \mu \mathrm{L}$} \\
\hline & $8 / 18 / 2010$ & Tumor: & & \\
\hline Time : & 2 Hour & Strain: & SCIDs & 5 mice \\
\hline Blocker: & Saline (I.V.) & & Dose Cts. & 541810.2 \\
\hline Organ & Avg \%ID & STDEV & Avg\%ID/g & STDEV \\
\hline Blood & 7.10 & 18.18 & 3.66 & 5.36 \\
\hline Heart & 0.05 & 0.08 & 0.34 & 0.65 \\
\hline Lung & 0.08 & 0.12 & 0.33 & 0.52 \\
\hline Liver & 3.71 & 3.67 & 2.23 & 2.78 \\
\hline Spleen & 0.43 & 0.51 & 6.52 & 6.98 \\
\hline Stomach & 3.07 & 2.19 & 4.22 & 3.34 \\
\hline L Int & 2.06 & 2.13 & 2.22 & 2.69 \\
\hline Sm Int & 2.79 & 3.38 & 1.80 & 2.36 \\
\hline Kidney & 0.22 & 0.41 & 0.67 & 1.08 \\
\hline Urine & 3.59 & 6.42 & & \\
\hline Abdominal Fat & 42.62 & 40.30 & 136.53 & 135.59 \\
\hline Muscle & 0.01 & 0.03 & 0.02 & 0.02 \\
\hline Bone & 0.00 & 0.00 & 0.05 & 0.08 \\
\hline Bladder & 0.21 & 0.39 & 7.74 & 12.10 \\
\hline Brain & 0.00 & 0.00 & 0.00 & 0.00 \\
\hline Pancreas & 0.36 & 2.16 & 7.79 & 9.06 \\
\hline Carcass & 39.80 & 30.12 & 21.84 & 2.09 \\
\hline Tumor A & 0.01 & 0.01 & 0.04 & 0.07 \\
\hline
\end{tabular}




\begin{tabular}{|c|c|c|c|c|}
\hline Compound: & \multicolumn{2}{|c|}{${ }^{198}$ Au-BBN-NP (I.P.) } & \multirow[b]{2}{*}{ PC-3 } & \multirow[t]{2}{*}{$130 \mathrm{kBq} / 100 \mu \mathrm{L}$} \\
\hline Date: & $8 / 18 / 2010$ & Tumor: & & \\
\hline Time : & 2 Hour & Strain: & SCIDs & 5 mice \\
\hline Blocker: & \multicolumn{2}{|c|}{ Cold BBN (I.V.) } & Dose Cts. & 541810.2 \\
\hline Organ & Avg \%ID & STDEV & Avg\%ID/g & STDEV \\
\hline Blood & 10.66 & 10.27 & 7.95 & 6.31 \\
\hline Heart & 0.02 & 0.02 & 0.08 & 0.20 \\
\hline Lung & 0.27 & 0.31 & 1.04 & 1.62 \\
\hline Liver & 3.50 & 3.49 & 3.19 & 2.89 \\
\hline Spleen & 0.75 & 0.78 & 8.06 & 12.04 \\
\hline Stomach & 2.26 & 2.50 & 4.45 & 6.23 \\
\hline L Int & 3.46 & 3.65 & 4.06 & 4.73 \\
\hline Sm Int & 5.06 & 5.12 & 3.78 & 3.28 \\
\hline Kidney & 0.65 & 0.91 & 1.76 & 3.00 \\
\hline Urine & 0.17 & 0.34 & & \\
\hline Abdominal Fat & 18.01 & 22.95 & 79.45 & 143.92 \\
\hline Muscle & 0.02 & 0.02 & 0.08 & 0.10 \\
\hline Bone & 0.00 & 0.00 & 0.06 & 0.07 \\
\hline Bladder & 0.09 & 0.08 & 1.78 & 3.13 \\
\hline Brain & 0.00 & 0.00 & 0.00 & 0.00 \\
\hline Pancreas & 0.56 & 0.56 & 9.87 & 16.93 \\
\hline Carcass & 61.58 & 34.05 & 9.99 & 1.69 \\
\hline Tumor A & 0.01 & 0.01 & 0.02 & 0.03 \\
\hline
\end{tabular}


Appendix 2: XRD scan parameters

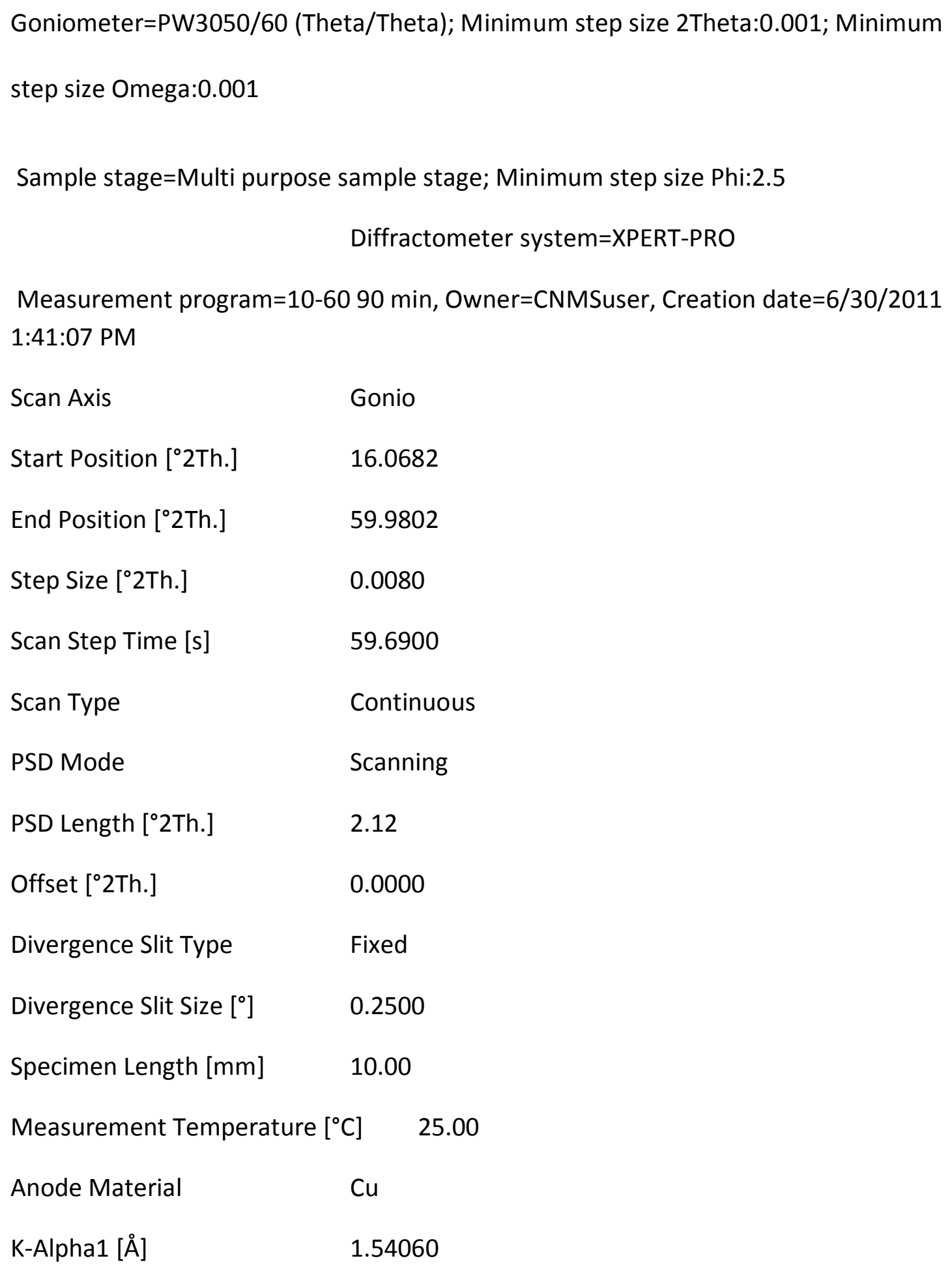




\begin{tabular}{|c|c|}
\hline K-Alpha2 [Å] & 1.54443 \\
\hline K-Beta $[\AA]$ & 1.39225 \\
\hline K-A2 / K-A1 Ratio & 0.50000 \\
\hline Generator Settings & $40 \mathrm{~mA}, 45 \mathrm{kV}$ \\
\hline Diffractometer Type & 0000000011010092 \\
\hline Diffractometer Number & 0 \\
\hline Goniometer Radius [mm] & 240.00 \\
\hline Dist. Focus-Diverg. Slit [mm] & 100.00 \\
\hline Incident Beam Monochromato & No \\
\hline Spinning & No \\
\hline
\end{tabular}


Appendix 3: Antibody:Nanoparticle Ratio Calculation

Nanoparticle mass:

Mass of individual NP = core volume (core density) + (layer volume) (layer density)

$=4 / 3 \pi\left(1.12^{*} 10^{\wedge}-6 \mathrm{~cm}\right)^{\wedge} 3\left(5 \mathrm{~g} / \mathrm{cm}^{\wedge} 3\right)+\left(4 / 3 \pi\left(1.17^{*} 10^{\wedge}-6 \mathrm{~cm}\right)^{\wedge} 3-4 / 3 \pi\right.$

$\left.\left(1.12^{*} 10^{\wedge}-6 \mathrm{~cm}\right)^{\wedge} 3\right)\left(19.3 \mathrm{~g} / \mathrm{cm}^{\wedge} 3\right)$

$=4.89 * 10^{\wedge}-18 \mathrm{~g} /$ nanoparticle

Number of nanoparticles:

Number of nanoparticles $=$ mass of nanoparticles/mass of individual nanoparticle

Mass of nanoparticles determined by synthetic conditions

$$
\begin{aligned}
& =0.00088 \mathrm{~g} / 4.89^{*} 10^{\wedge}-18 \mathrm{~g} \\
& =1.8^{*} 10^{\wedge} 14 \text { nanoparticles }
\end{aligned}
$$

Antibody mass determined by Lowry assay or ${ }^{125} \mathrm{I}$ radiolabeling

Panitumumab antibodies = mass mAb/molecular weight mAb * Avogadro's number

$=0.00019 \mathrm{~g} / 150,000 \mathrm{~g} / \mathrm{mol} * 6.022 * 10^{\wedge} 23 \mathrm{molecules} / \mathrm{mol}$

$=7.63^{*} 10^{\wedge} 14$ antibodies

Ratio of panitumumab:nanoparticle:

$7.63^{*} 10^{\wedge} 14$ antibodies $/ 1.8^{*} 10^{\wedge} 14$ nanoparticles $=4.2$ antibodies/nanoparticle 


\section{BIBLIOGRAPHY}

${ }^{1}$ American Cancer Society. Cancer Facts and Figures 2012. http://www.cancer.org/acs/groups/content/@epidemiologysurveilance/documents/do cument/acspc-031941.pdf (accessed 11/28/12).

${ }^{2}$ Bray, F.; Jemal, A.; Grey, N.; Ferlay, J.; Forman, D. Lancet Oncol. 2012, 13, 790.

${ }^{3}$ Rice, J. Nature 2012, 485, S55.

${ }^{4}$ Parise, C.A.; Bauer, K.R.; Brown, M.M.; Caggiano, V. Breast J. 2009, 15, 594.

${ }^{5}$ Tan, A.R.; Swain, S.M. Semin. Oncol. 2003, 30, 54.

${ }^{6}$ Elad, S.; Zadik, Y.; Hewson, I.; Hovan, A; Correa, M.E.; Logan, R.; Elting, L.S.; Spijkervet, F.K.; Brennan, M.T. Support. Care. Cancer 2010, 18, 993.

${ }^{7}$ Keidan, R.D.; Fanning, J.; Gatenby, R.A.; Weese, J.L. Dis. Colon Rectum 1989, 32, 206.

${ }^{8}$ Gralla, R.J.; De Wit, R.; Herrstedt, J.; Carides, A.D.; Ianus, J.; Guoguang-Ma, J.; Evans, J.K.; Horgan, K.J. Cancer 2005, 104, 864.

${ }^{9}$ Bucci, M.; Bevan, A.; Roach, M. Cancer J. Clin. 2005, 55, 117.

${ }^{10}$ Koukourakis, G.; Kelekis, N.; Armonis, V.; Kouloulias, V. Adv. Urol. 2005, 1.

${ }^{11}$ Litwin, M.S.; Gore, J.L.; Kwan, L; Brandeis, J.M.; Lee, S.P.; Withers, H.R.; Reiter, R.E. Cancer. 2007, 109, 2239.

12 Jacene, H.A.; Filice, R.; Kasecamp, W.; Wahl, R.W. J. Nucl. Med. 2007, 48, 1767.

${ }^{13}$ Milenic, D.E.; Brady, E.D.; Brechbiel, M.W. Nat. Rev. Drug Discov. 2004, 3, 488.

14 Lliakis, G. BioEssays 1991, 13, 641.

${ }^{15}$ Hall, E.J. Radiobiology for the Radiologist. 5th ed. Lippincott Williams \& Wilkins: Philadelphia, PA, 2000.

${ }^{16}$ Gadbois, D.M.; Crissman, H.A.; Nastasi, A.; Habbersett, R.; Wang, S.K.; Chen, D.; Lehnert, B.E. Radiat. Res. 1996, 146, 414.

${ }^{17}$ Harrison, L.B.; Chadha, M.; Hill, R.J.; Hu, K.; Shasha, D. Oncologist 2002, 7, 492.

${ }^{18}$ Raju, M.R.; Eisen, Y.; Carpenter, S.; Inkret, W.C. Radiat. Res. 1991, 128, 204.

${ }^{19}$ Vaidyanathan, G.; Zalutsky, M.R. Curr. Radiopharm. 2011, 4, 283.

${ }^{20}$ Enger, S.A.; Hartman, T.; Carlsson, J.; Lundqvist, H. Phys. Med. Biol. 2008, 53, 1909.

${ }^{21}$ Brechbiel, M.W. Q. J. Nucl. Med. Mol. Imaging 2008, 52, 166.

${ }^{22}$ Moghimi, S.M.; Hunter, A.C.; Andresen, T.L. Annu. Rev. Pharmacol. Toxicol. 2012, 52, 481.

${ }^{23}$ Mankoff, D.A.; Link, J.M.; Linden, H.M.; Sundararajan, L.; Krohn, K.A. J. Nucl. Med. 2008, 49, 149S.

${ }^{24}$ Bhattacharyya, S.; Khan, J.A.; Curran, G.L; Robertson, J.D.; Bhattacharya, R.; Mukherjee, P. Adv. Mater. 2011, 23, 5034. 
${ }^{25}$ Maeda, H. Advan. Enzyme Regul. 2001, 41, 189.

${ }^{26}$ Li, L.; Ten Hagen, T.L.; Bolkestein, M.; Gasselhuber, A.; Yatvin, J.; van Rhoon, G.C.; Eggermont, A.M.; Haemmerich, D.; Koning, G.A. J. Control. Release. 2013, in press.

${ }^{27}$ Turkevich, J.; Stevenson, P.C.; Hillier, J. Discuss. Faraday. Soc. 1951, 11, 55.

${ }^{28}$ Brust; M.; Walker; M.; Bethell, D.; Schiffrin, D.J.; Whyman, R. Chem. Commun. 1994, 7, 801.

${ }^{29}$ Hoskins, C.; Min, Y.; Gueorguieva, M.; McDougall, C.; Volovick, A.; Prentice, P.; Wang, Z.; Melzer, A.; Cuschieri, A.; Wang, L. J. Nanobiotechnology 2012, 10, 27.

${ }^{30}$ Pacific Northwest National Laboratory. Isotope Sciences Program : Thorium-227. http://radioisotopes.pnnl.gov/thorium227.stm (Accessed 12/5/12).

${ }^{31}$ Boll, R.A.; Mirzadeh, S.; Kennel, S.J.; DePaoli, D.W.; Webb, O.F. J. Labelled Comp. Radiopharm 1997, 40, 341.

${ }^{32}$ Apostolidis, C.; Molinet, R.; McGinley, J.; Abbas, K.; Möllenbeck, J.; Morgenstern, A. Appl. Radiat. Isot. 2005, 62, 383.

${ }^{33}$ Weidner, J.W.; Mashnik, S.G.; John, K.D.; Ballard, B.; Birnbaum, E.R.; Bitteker, L.J.; Couture, A.; Fassbender, M.E.; Goff, G.S.; Gritzo, R.; Hemez, F.M.; Runde, W.; Ullmann, J.L.; Wolfsberg, L.E.; Nortier, F.M. Appl. Radiat. Isot. 2012, 70, 2590.

${ }^{34}$ Hagemann, F. J Am. Chem. Soc. 1950, 72, 768.

${ }^{35}$ Kirby, H.W.; Grove, G.R.; Timma, D.L. Phys. Rev. 1956, 102, 1140.

${ }^{36}$ Mirzadeh, S.; Kumar, K.; Gansow, O.A. Radiochim. Acta 1993, 60, 1.

${ }^{37}$ Bruland, O.S.; Nilsson, S.; Fisher, D.R.; Larsen, R.H. Clin. Cancer. Res. 2006, 12, 6250s.

${ }^{38}$ Medical news today. "FDA Grants Fast Track Designation For Alpharadin For Castration Resistant Prostate Cancer In Patients With Bone Metastases." 2011 http://www.medicalnewstoday.com/articles/233215.php (accessed 8/18/12)

${ }^{39}$ Jaggi, J.S.; Kappel, B.J.; McDevitt, M.R.; Sgouros, G.; Flombaum, C.D.; Cabassa, C.; Scheinberg, D.A. Cancer Res. 2005, 65, 4888.

${ }^{40}$ Essler, M.; Gärtner, F.C.; Neff, F.; Blechert, B.; Senekowitsch-Schmidtke, R.; Bruchertseifer, F.; Morgenstern, A.; Seidl, C. J. Nucl. Med. Mol. Imaging. 2012, 39, 602.

${ }^{41}$ Song, H.; Hobbs, R.F.; Vajravelu, R.; Huso, D.L.; Esaias, C.; Apostolidis, C.; Morgenstern, A.; Sgouros, G. Cancer Res. 2009, 69, 8941.

${ }^{42}$ Sofou, S.; Thomas, J.L.; Lin, H.; McDevitt, M.R.; Scheinberg, D.A.; Sgouros, G. J. Nucl. Med. 2004, 45, 253.

${ }^{43}$ Chang, M.; Seideman, J.; Sofou, S. Bioconjugate Chem. 2008, 19, 1274.

${ }^{44}$ Thijssen, L.; Schaart, D.R.; de Vries, D.; Morgenstern, A.; Bruchertseifer, F.; Denkova, A.G. Radiochim. Acta. 2012, 100, 473.

${ }^{45}$ Brigger, I.; Dubernet, C.; Couvreur, P. Adv. Drug Deliv. Rev. 2002, 54, 631.

${ }^{46}$ Brannon-Peppas, L.; Blanchette, J.O. Adv. Drug Deliv. Rev. 2004, 56, 1649.

${ }^{47}$ Mwakisege, J.; Mirzadeh, S.; Boll, R.A.; Schweitzer, G. Synthesis and chemical stability of actinium-fullerenes. Presented at the $234^{\text {th }}$ American Chemical Society National Meeting, Boston, MA (2007). 
${ }^{48}$ Woodward, J.; Kennel, S.J.; Stuckey, A.; Osborne, D.; Wall, J.; Rondinone, A.J.;

Standaert, R.F.; Mirzadeh, S. Bioconjugate Chem. 2011, 22, 776.

${ }^{49}$ McLaughlin, M.F.; Woodward, J.; Boll, R.A.; Rondinone, A.J.; Mirzadeh, S.; Robertson, J.D. PLOS ONE 2013, 8, e54531.

${ }^{50}$ Ziegler, J. SRIM/TRIM, version SRIM-2008; http://www.srim.org/ (accessed November 2010).

${ }^{51}$ ClinicalTrials.gov. Targeted atomic nano-generators (Actinium-225-labeled humanized anti-CD33 monoclonal antibody HuM195) in patients with advanced myeloid malignancies. July 23, 2012. http://clinicaltrials.gov/show/NCT00672165. (Accessed 12/6/2012).

${ }^{52}$ ClinicalTrials.gov. Low dose cytarabine and lintuzumab-Ac225 in older patients. April 17, 2013. http://clinicaltrials.gov/ct2/show/NCT01756677?term=actinium-225\&rank=2. (Accessed 4/21/2013).

${ }^{53}$ Baum, E.; Knox, H.; Miller, T. Nuclides and Isotopes: Chart of the Nuclides, $16^{\text {th }}$ ed.; Lockheed Martin, Bathesda, MD, 2002.

${ }^{54}$ Table of Radioactive Isotopes. http://ie.Ibl.gov/toi/radSearch.asp (accessed April 3, 2010).

${ }^{55}$ Brockman, J. University of Missouri Research Reactor, Columbia, MO. Unpublished work, 2010.

${ }^{56}$ Kolsky, K. L.; Mausner, L. F. Appl. Radiat. Isot. 1993, 44, 553.

${ }^{57}$ Kan, Para. Radioactive Au for Potential Radiotherapeutic Application. Master's Thesis, University of Missouri-Columbia, Columbia, MO, 2010.

${ }^{58}$ Bard, A.J; Parsons, R.; Jordan, J. Standard Potentials in Aqueous Solutions, IUPAC, New York, USA, 1985.

${ }^{59}$ Bottenus, B.N.; Kan, P.; Jenkins, T.; Ballard, B.; Rold, T.L.; Barnes, C.; Cutler, C.; Hoffman, T.J.; Green, M.A.; Jurisson, S.S. Nucl. Med. Biol. 2010, 37, 41.

${ }^{60}$ Casas, J.S.; Castellano, E.E.; Couce, M.D.; Ellena, J.; Sanchez, A.; Sordo, J.; Taboada, C. J. Inorg. Biochem. 2006, 100, 1858.

${ }^{61}$ Obata, A.; Kasamatsu, S.; McCarthy, D.W.; Welch, M.J.; Saji, H.; Yonekura, Y.;

Fujibayashi, Y. Nucl. Med. Biol. 2003, 30, 535.

${ }^{62}$ Anderson, C.J.; Ferdani, R. Cancer Biother. Radiopharm. 2009, 24, 379.

${ }^{63}$ Reile, H.; Armatis, P.E.; Schally, A.V. The Prostate 1994, 25, 29.

${ }^{64}$ Ananias, H.J.K.; Yu, Z.; Dierckx, R.A.; van der Wiele, C.; Helfrich, W.; Wang, F.; Yan, Y.; Chen, X.; de Jong, I.J.; Elsinga, P.H. Mol. Pharm. 2011, 8, 1165.

${ }^{65}$ Cooper, M.S.; Ma, M.T.; Sunassee, K.; Shaw, K.P.; Williams, J.D.; Paul, R.L.; Donnelly, P.S.; Blower, P.J. Bioconjugate Chem. 2012, 23, 1029.

${ }^{66}$ Chanda, N.; Kan, P.; Watkinson, L.D.; Shukla, R.; Zambre, A.; Carmack, T.L.; Engelbrecht, H.; Lever, J.R.; Katti, K.; Fent, G.M.; Casteel, S.W.; Smith, C.J.; Miller, W.H.; Jurisson, S.; Boote, E.; Robertson, J.D.; Cutler, C.; Dobrovolskaia, M.; Kannan, R.; Katti, K.V. Nanomedicine. 2010, 6, 201. 
${ }^{67}$ Kannan, R.; Zambre, A.; Chanda, N.; Kulkarni, R.; Shukla, R.; Katti, K.; Upendran, A.; Cutler, C.; Boote, E.; Katti, K.V. WIREs Nanomed. Nanobiotechnol. 2012, 4, 42.

${ }^{68}$ Alkilany, A.M.; Murphy, C.J. J. Nanopart. Res. 2010, 12, 2313.

${ }^{69}$ Goodman, C.M.; McCusker, C.D.; Yilmaz, T.; Rotello, V.M. Bioconjugate Chem. 2004, $15,897$.

${ }^{70}$ Lane, S. R.; Nanda, P.; Rold, T.L.; Sieckman, G.L.; Figueroa, S.D.; Hoffman, T.J.; Jurisson, S.S.; Smith, C.J. Nucl. Med. Biol. 2010, 37, 751.

${ }^{71}$ Chanda, N.; Kattumuri, V.; Shukla, R.; Zambre, A.; Katti, K.; Upendran, A.; Kulkarni, R.R.; Kan, P.; Fent, G.M.; Casteel, S.W.; Smith, C.J.; Boote, E.; Robertson, J.D.; Cutler, C.; Lever, J.R.; Katti, K.V.; Kannan, R. Proc. Natl. Acad. Sci. 2010, 107, 8760.

${ }^{72}$ Huang, S.C.; Yu, D.H.; Wank, S.A.; Gardner, J.D.; Jensen, R.T. Peptides 1990, 11, 1143.

${ }^{73}$ National Institute of Standards and Technology. http://www.itl.nist.gov/div898/handbook/eda/section3/eda35h.htm (Accessed 2/18/2013)

${ }^{74}$ Chanda, N.; Kattumuri, V.; Shukla, R.; Zambre, A.; Katti, K.; Upendran, A.; Kulkarni, R.R.; Kan, P.; Fent, G.M.; Casteel, S.W.; Smith, C.J.; Boote, E.; Robertson, J.D.; Cutler, C.; Lever, J.R.; Katti, K.V.; Kannan, R. Proc. Natl. Acad. Sci. 2010, 107, 8760.

${ }^{75}$ Wolfrom, M.L.; Myers, D.R.; Lassettre, E.N. J. Am. Chem. Soc. 1939, 61, 2172.

${ }^{76}$ Zhang, S.; Leem, G.; Srisombat, L.; Lee, T.R. J. Am. Chem. Soc. 2008, 130, 113.

${ }^{77}$ Subramanian, G.; Schneider, R.F. (Research Foundation of the State of New York). Metal chelating agents for medical application. US Patent Number 5,463,030, October 31, 1995.

${ }^{78}$ Debouttière, P.J; Roux, S.; Vocanson, F.; Billotey, C.; Beuf, O.; Favre-Réguillon, A.; Lin, Y.; Pellet-Rostaing, S.; Lamartine, R.; Perriat, P.; Tillement, O. Adv. Funct. Mater. 2006, 16, 2330.

${ }^{79}$ McCarthy, D.W.; Shefer, R.E.; Klinkowstein, R.E.; Bass, L.A.; Margeneau, W.H.; Cutler, C.S.; Anderson, C.J.; Welch, M.J. Nucl. Med. Biol. 1997, 24, 35.

${ }^{80}$ McLaughlin, M.F.; Kelsey, J.B.; Zambre, A.; Carmack, T.; Watkinson, L.; Kannan, K.; Cutler, C.S. University of Missouri Research Reactor, Columbia, MO. Unpublished work, 2011

${ }^{81}$ Greenwood, R.; Kendall, K. J. Eur. Ceram. Soc. 1999, 19, 479.

${ }^{82}$ Stipanuk, M.H.; Dominy Jr., J.E.; Lee, J.I.; Coloso, R.M. J. Nutr. 1996, 136, 1652 S.

${ }^{83}$ Shokeen, M.; Anderson, C.J. Acc. Chem. Res. 2009, 42, 832.

${ }^{84}$ McMillin, G.A.; Travis, J.J.; Hunt, J.W. Am. J. Clin. Pathol. 2009, 131, 160.

${ }^{85}$ Guyton, A.C.; Hall, J.E. Textbook of Medical Physiology. Elsevier Saunders: Philadelphia, PA, 1996.

${ }^{86}$ Bush, J.A.; Mahoney, J.P.; Markowitz, H.; Gubler, C.J.; Cart-Wright, G.E.; Wintrobe, M.M. J. Clin. Invest. 1955, 34, 1766.

${ }^{87}$ Albanese, A.; Tang, P.S.; Chan, W.C.W. Annu. Rev.Biomed.Eng. 2012, $14,1$.

${ }^{88}$ Weiss, C.J.; Marks, T.J. Dalton Trans. 2010, 39, 6576.

${ }^{89}$ Choppin, G.R. J. Less-Common Met. 1983, 93, 323. 
${ }^{90}$ Christiansen, P.A. Ann. Rev. Phys. Chem. 1985, 36, 407.

${ }^{91}$ Firsching, F.H.; Brune, S.N. J. Chem. Eng. Data. 1991, 36, 93.

92 Boll, R.A.; Malkemus, D.; Mirzadeh, S. Appl. Radiat. Isot. 2005, 62, 667.

${ }^{93}$ Buissette, V.; Giaume, D.; Gacoin, T.; Boilot, J.P. J. Mater. Chem. 2006, 16, 529.

${ }^{94}$ Meiser. F.; Cortez, C.; Caruso, F. Angew. Chem. Int. Edit. 2004, 43, 5954.

${ }^{95}$ Li, D.; Nielsen, M.H.; Lee, J.R.I.; Frandsen, C.; Banfield, J.F.; De Yoreo, J.J. Science 2012, 336, 1014.

${ }^{96}$ Hifumi, H.; Yamaoka, S.; Tanimoto, A.; Akatsu, T.; Shindo, Y.; Honda, A.; Citterio, D.; Oka, K.; Kuribayashib, S.; Suzuki, K. J. Mater. Chem. 2009, 19, 6393.

97 Shannon, R.D. Acta Cryst. A. 1976, 32, 751.

98 Mirzadeh, S.; Kumar, K.; Gansow, O.A. Radiochim. Acta 1993, 60, 1.

${ }^{99}$ Lowry, O.H.; Rosebrough, N.J.; Farr, A.L.; Randall, R.J. J. Biol. Chem. 1951, 193, 265.

${ }^{100}$ Kennel SJ, Huang Y, Zeng WB, Stuckey A, Wall J. Kinetics of vascular targeted monoclonal antibody. Curr. Drug Deliv. 2010;7:428.

${ }^{101}$ Kennel, SJ, Chappell, LL, Dadachova, K, Brechbiel, MW, Lankford, TK, Davis, IA, Stabin, $\mathrm{M}$, Mirzadeh, S. Evaluation of ${ }^{225} \mathrm{Ac}$ for vascular targeted radioimmunotherapy of lung tumors. Cancer Biother. Radio. 2000;15:235.

${ }^{102}$ An, Z. Therapeutic Monoclonal Antibodies: From Bench to Clinic. John Wiley and Sons: Hoboken, NJ, 2011.

${ }^{103}$ Schwartz, J.; Jaggi, J.S.; O’Donoghue, J.A.; Ruan, S.; McDevitt, M.; Larson, S.M.; Scheinberg, D.A.; Humm, J.L. Phys. Med Biol. 2011, 56, 721.

${ }^{104}$ Kennel, S.J.; Woodward, J.D.; Rondinone, A.J.; Wall, J.; Huang, Y.; Mirzadeh, S. Nucl. Med. Biol. 2008, 35, 501.

${ }^{105}$ Vanrooijen, N. J. Immunol. Methods 1989, 124, 1.

${ }^{106}$ Lasbury, M.E.; Durant, P.J.; Ray, C.A.; Tschang, D. Schwendener, R.; Lee, C.H. Immunol. 2006, 176, 6443.

${ }^{107}$ Kennel, S.J.; Chappell, L.L.; Dadachova, K.; Brechbiel, M.W.; Lankford, T.K.; Davis, I.A.; Stabin, M.; Mirzadeh, S. Cancer Biother. Radiopharm. 2000, 15, 235.

${ }^{108}$ Roney, C.; Kulkarni, P.; Arora, V.; Antich, P.; Bonte, F.; Wu, A.; Mallikarjuana, N.N.; Manohar, S.; Liang, H.F.; Kulkarni, A.R.; Sung, H.W.; Sairam, M.; Aminabhavi, T.M. J.

Control. Release. 2005, 108, 193.

${ }^{109}$ Yang, H. Pharm. Res. 2010, 27, 1759.

${ }^{110}$ Wang, M.; Etu, J.; Joshi, S. J. Neurosurg. Anesthesiol. 2007, 19, 249.

${ }^{111} \mathrm{Hu}$, F.; Cutler, C.S.; Hoffman, T.; Sieckman, G.; Volkert, W.A.; Jurisson, S.S. Nucl. Med. Biol. 2002, 29, 423.

${ }^{112}$ Simon, J.; Goeckeler, W.F.; Edwards, B.; Stringham, L.; Volkert, W.A.; Troutner, D.E.; Holmes, R.A. J. Labl. Compd. Radiopharm. 1986, 23, 1344.

${ }^{113}$ De Silva, C.R.; Vagner, J.; Lynch, R.;Gillies, R.J.; Hruby, V.J. Anal. Biochem.2010, 398, 15.

${ }^{114}$ Ferreira Soares, D.C.; de Oliveira, M.C.; Branco de Barros, A.L.; Cardoso, V.N.; Ramaldes, G.A. Eur. J. Pharm. Sci. 2011, 43, 290. 
${ }^{115}$ Lehenberger, S.; Barkhausen, C.; Cohrs, S.; Fischer, E.; Grünberg, J.; Hohn, A.; Köster, U.; Schibli, R.; Türler, A.; Zhernosekov, K. Nucl. Med. Biol. 2011, 38, 917.

${ }^{116}$ Ferro-Flores, G.; Hernández-Oviedo, O.; Arteaga de Murphy, C.; Tendilla, J.I.; MonroyGuzmán, F.; Pedraza-López, M.; Aldama-Alvarado, K. Appl. Radiat. Isot. 2004, 61, 1227.

${ }^{117}$ Kahan A, Mödder G, Menkes CJ, Verrier P, Devaux JY, Bonmartin A, De Rycke Y, Manil L, Chossat F, Tebib J. Clin. Exp. Rheumatol. 2004, 22, 722.

118 Mathew, B.; Chakraborty, S.; Das, T.; Sarma, H.D.; Banerjee, S.; Samuel, G.; Venkatesh, M.; Pillai, M.R. Appl. Radiat. Isot. 2014, 60, 635.

${ }^{119}$ Gains, J.E.; Bomanji, J.B.; Fersht, N.L.; Sullivan, T.; D'Souza, D.; Sullivan, K.P.; Aldridge, M.; Waddington, W.; Gaze, M.N. J. Nucl. Med. 2011, 52, 1041.

${ }^{120}$ Buissette, V.; Moreau, M.; Gacoin, T.; Boilot, J.P.; Chane-Ching, J.Y.; Le Mercier, T. Chem. Mater. 2004, 16, 3767.

${ }^{121}$ Wada, S.; Kondo, S.; Moriyoshi, C.; Kuroiwa, Y. Jpn. J. Appl. Phys. 2008, 47, 7612.

122 Petrova, A.; Hintz, W.; Tomas, J. Chem. Eng. Technol. 2008, 31, 604.

${ }^{123}$ Gonzalez-Carreno, T.; Morales, M.P.; Serna, C.J. Mater. Lett. 2000, 43, 97.

${ }^{124}$ Buissette, V.; Moreau, M.; Gacoin, T.; Boilot, J.P. Adv. Funct. Mater. 2006, 16, 351.

${ }^{125}$ Horning, S.J.; Younes, A.; Jain, V.; Kroll, S.; Lucas, J.; Podoloff, D.; Goris, M. J. Clin. Oncol. 2005, 23, 712.

${ }^{126}$ Friesen, C.; Glatting, G.; Koop, B.; Schwarz, K.; Morgenstern, A.; Apostolidis, C.;

Debatin, K.M.; Reske, S.N. Cancer Res. 2007, 67, 1950.

${ }^{127}$ Press, O.W.; Appelbaum, F.; Ledbetter, J.A.; Martin, P.J.; Zarling, J.; Kidd, P.; Thomas, E.D. Blood. 1987, 69, 584.

${ }^{128}$ Pagel, J.M.; Appelbaum, F.R.; Eary, J.F.; Rajendran, J.; Fisher, D.R.; Gooley, T.; Ruffner, K.; Nemecek, E.; Sickle, E.; Durack, L.; Carreras, J.; Horowitz, M.M.; Press, O.W.; Gopal, A.K.; Martin, P.J.; Bernstein, I.D.; Matthews, D.C. Blood. 2006, 107, 2184.

${ }^{129}$ WebMD. Peritoneal Cancer. http://www.webmd.com/cancer/peritoneal-cancerprognosis-symptoms-treatments (Accessed 3/7/13).

${ }^{130}$ Lu, Z.; Wang, J.; Wientjes, M.G.; L-S Au, J. Future Oncol. 2010, 6, 1625. 
Mark Francis McLaughlin was born February 22, 1987 to Donald and Cheryl McLaughlin. He grew up in Lee's Summit, MO and graduate valedictorian from Lee's Summit High School. Following graduation, he attended the University of Notre Dame in South Bend, IN, earning degrees in chemistry and philosophy. Summer research experiences radiochemistry at the University of Missouri and Brookhaven National Laboratory sparked an interest in radiopharmaceutical research. He returned to Missouri in 2009 to begin work on his Ph.D., defending his dissertation in 2013. Following his doctorate, Mark will attend medical school to gain more insight into the biological basis of disease before pursuing a career as a medical research scientist and clinician. 